\title{
THE GAUGING OF TWO-DIMENSIONAL BOSONIC SIGMA MODELS ON WORLD-SHEETS WITH DEFECTS
}

\author{
KRZYSZTOF GAWȨDZKI, RAFAŁ R. SUSZEK, AND KONRAD WALDORF
}

\begin{abstract}
We extend our analysis of the gauging of rigid symmetries in bosonic twodimensional sigma models with Wess-Zumino terms in the action to the case of worldsheets with defects. A structure that permits a non-anomalous coupling of such sigma models to world-sheet gauge fields of arbitrary topology is analysed, together with obstructions to its existence, and the classification of its inequivalent choices.
\end{abstract}

\section{Contents}

1. Introduction

2. Preliminaries

2.1. The geometric structure of the $\sigma$-model

2.2. G-actions via simplicial G-spaces

2.3. Rigid symmetries of the $\sigma$-model

3. The coupling to gauge fields: topologically trivial sector 16

3.1. Insights from the study of trivial backgrounds 16

3.2. An Ansatz for nontrivial backgrounds, and constraints for a consistent gauging 19

4. Large gauge transformations in the topologically trivial sector 23

5. An example of a string background: the WZW model 29

5.1. The WZW target 29

5.2. The boundary maximally symmetric WZW bi-branes 30

5.3. The non-boundary maximally symmetric WZW bi-branes 31

5.4. The maximally symmetric WZW inter-bi-brane 32

5.5. Global gauge anomalies of the WZW-model amplitudes 33

6. A groupoidal interpretation of the constraints 35

7. The gauging vs. the $\mathfrak{g}$-equivariance of string backgrounds 36

7.1. The local description of the background structure 36

7.2. $\mathfrak{g}$-equivariant structures 38

8. G-equivariant string backgrounds 39

8.1. G-equivariant structures 40

8.2. The descent principle for G-equivariant string backgrounds 43

9. Coset $\sigma$-models 47

10. The coupling to arbitrary gauge fields 51

10.1. Equivariance properties of P-extended string backgrounds 52

10.2. Fully gauged $\sigma$-models and their gauge invariance 57

11. The cohomological classification of G-equivariant string backgrounds

11.1. Setting up the local description 5

11.2. $(\mathrm{G}, \rho)$-equivariant gerbes 63

11.3. $(\mathrm{G}, \lambda)$-equivariant bi-branes 68

11.4. G-equivariant inter-bi-branes 72

12. Conclusions 74

Appendix A. A proof of the "only if" part of Proposition 2.24

Appendix B. Proofs of the "only if" parts of Proposition 4.9 and Theorem 4.12

Appendix C. Groupoids and algebroids 79

Appendix D. A proof of Theorem 6.4 80

Appendix E. A proof of Proposition 7.2

Appendix F. A proof of Proposition 7.3

Key words and phrases. Gauged sigma models; Defects; Gerbes. 
Appendix G. A proof of Proposition 7.4

Appendix H. Commuting diagrams for G-equivariant string backgrounds

Appendix I. Natural simplicial refinements of open covers

\section{INTRODUCTION}

In this paper, we study a non-anomalous gauging of rigid symmetries in two-dimensional bosonic non-linear $\sigma$-models with Wess-Zumino terms in the action functional. Classical fields of such models take values in target manifolds equipped with appropriate differentialgeometric structures. We shall admit the presence of defects at which the values of fields jump or change the target space. Rigid symmetries of such $\sigma$-models are induced by transformations of the target spaces that may be consistently lifted to the differential-geometric structures required for the definition of the action functional, e.g. a Riemannian metric, a gerbe, or a gerbe bi-module. In this context, we present a detailed study of the circumstances under which rigid symmetries may be promoted to local ones by a gauging procedure that couples the original fields of the model to world-sheet gauge fields. Our study leads to a formulation of necessary conditions for the consistent gauging and to an exhaustive cohomological classification of the resulting gauged $\sigma$-models. In particular, we cover in a unified way the gauging of continuous symmetries and, for discrete symmetries, the known description of orbifold $\sigma$-models.

An analogous program was realized in Ref. GSW10 in the simpler case of the mono-phase $\sigma$-model. Completing the earlier studies of the subject [JJMO90, HS91, FOS94a, FOS94b, that work also covered the case of gauge fields in nontrivial principal bundles of the symmetry group $\mathrm{G}$ and provided an exhaustive study of global gauge anomalies that may obstruct invariance under 'large' gauge transformations non-homotopic to the identity. The interested reader may consult Ref. GSW10 for a description of the physical background and for a more detailed account of the history of the topic. The main point of the present work is to extend the analysis of Ref. GSW10 to the case of multi-phase $\sigma$-models in which the different phases of the two-dimensional theory are separated by one-dimensional domain walls, called defect lines that may intersect at defect junctions. Our analysis will lead to a natural completion of the analysis of Ref. GSW10 that accommodates the full-blown 2-categorial background of a general $\sigma$-model characterised in Refs. [FSW08, RS09, Sus11]. In application to boundary defects, it extends the results of Ref. FOM05| by treating the case of gauge fields in nontrivial principal bundles and by analyzing the global gauge anomalies in boundary $\sigma$-models with Wess-Zumino terms.

The paper is organized as follows. In Section 2, we briefly recall the mathematical structures involved in the definition of Feynman amplitudes of the $\sigma$-models with defects and in the description of their rigid symmetries. Section 3 describes the coupling of the $\sigma$-model to gauge fields in a trivial principal bundle of the symmetry group, extending the approach of Ref. JJMO90, HS91, FOM05 to the case of $\sigma$-models with defects. The resulting coupling assures the invariance of the gauged amplitudes under infinitesimal gauge transformations, i.e. the absence of local gauge anomalies. In Section 4 , we discuss the behaviour of the gauged amplitudes under large gauge transformations, completing the analysis of global gauge anomalies for topologically trivial gauge fields performed in Ref. GSW10 for $\sigma$-models without defects. In Section 5, we illustrate the preceding discussions on the example of the WZW model with defects. Sections 6 and 7 are an interlude providing an interpretation of the conditions assuring the absence of local gauge anomalies in terms of (Lie) algebroids related to generalised geometry and in terms of mixed Deligne and Lie-algebraic cohomology. Section 8 is devoted to an extension of the notion of a G-equivariant structure on gerbes, introduced in Ref. GSW10, to the rest of the geometric structure needed to define Feynman amplitudes for a $\sigma$-model with defects. In Section 9, the definition of the full-fledged G-equivariant structure is motivated by showing how it permits to push down the target-space structure to the quotient of the target by the symmetry group G whenever the quotient space is smooth, and in Section 10, it is shown how a G-equivariant structure permits to couple the $\sigma$-model with defects to world-sheet gauge fields in an arbitrary principal G-bundle. Finally, Section 
11 provides a detailed study of obstructions to the existence and the classification of Gequivariant structures, extending the results of Ref. GSW10 to the case with defects. Nine Appendices contain a few more technical proofs, as well as some additional material.

Acknowledgements: R.R.S. was partially funded by the Collaborative Research Centre 676 "Particles, Strings and the Early Universe - the Structure of Matter and Space-Time" at the beginning of the work, and subsequently from the Polish Ministry of Science and Higher Education grant No. N N201 372736. R.R.S. and K.W. are grateful to the Laboratoire de Physique at ENS-Lyon for hospitality generously extended to them at various stages of the work on the project reported herein.

\section{Preliminaries}

2.1. The geometric structure of the $\sigma$-model. Let us first recall some basic notions on which the subsequent analysis of the $\sigma$-models with Wess-Zumino terms and defects is founded. We start by identifying, after Ref. [FSW08, RS09], the differential-geometric structure on the target space underlying the definition of the Feynman amplitudes of the field theory of interest.

Definition 2.1. A string background is a triple $\mathfrak{B}=(\mathcal{M}, \mathcal{B}, \mathcal{J})$ composed of the following structure:

(1) the target $\mathcal{M}=(M, \mathrm{~g}, \mathcal{G})$ consisting of a smooth manifold $M$ called the target space, a metric g, and a bundle gerbe $\mathcal{G}$;

(2) the $\mathcal{G}$-bi-brane $\mathcal{B}=\left(Q, \iota_{1}, \iota_{2}, \omega, \Phi\right)$ consisting of a smooth manifold $Q$ called the $\mathcal{G}$-bi-brane world-volume, a 2 -form $\omega$ called the $\mathcal{G}$-bi-brane curvature, two smooth maps $\iota_{1}, \iota_{2}: Q \rightarrow M$, and a gerbe 1-isomorphism

$$
\Phi: \iota_{1}^{*} \mathcal{G} \stackrel{\cong}{\rightarrow} \iota_{2}^{*} \mathcal{G} \otimes I_{\omega},
$$

written in terms of the trivial gerbe $I_{\omega}$ with curving $\omega$;

(3) a collection $\mathcal{J}=\left(\mathcal{J}_{3}, \mathcal{J}_{4}, \mathcal{J}_{5}, \ldots\right)$ in which $\mathcal{J}_{n}$ is the $n$-valent $(\mathcal{G}, \mathcal{B})$-inter-bi-brane

$$
\mathcal{J}_{n}=\left(T_{n} ; \varepsilon_{n}^{1,2}, \varepsilon_{n}^{2,3}, \ldots, \varepsilon_{n}^{n-1, n}, \varepsilon_{n}^{n, 1} ; \pi_{n}^{1,2}, \pi_{n}^{2,3}, \ldots, \pi_{n}^{n-1, n}, \pi_{n}^{n, 1} ; \varphi_{n}\right),
$$

consisting of

(a) a smooth manifold $T_{n}$.

(b) maps $\varepsilon_{n}^{*, *}: T_{n} \rightarrow\{-1,1\}$ called the orientation maps;

(c) smooth maps $\pi_{n}^{*, *}: T_{n} \rightarrow Q$ subject to the constraints

$$
\iota_{2}^{\varepsilon_{n}^{k-1, k}} \circ \pi_{n}^{k-1, k}=\iota_{1}^{\varepsilon_{n}^{k, k+1}} \circ \pi_{n}^{k, k+1}=: \pi_{n}^{k}, \quad k \in \mathbb{Z} / n \mathbb{Z},
$$

where $\iota_{1}^{+1}:=\iota_{1}, \iota_{2}^{+1}:=\iota_{2}, \iota_{1}^{-1}:=\iota_{2}$ and $\iota_{2}^{-1}:=\iota_{1}$

(d) a gerbe 2-isomorphism

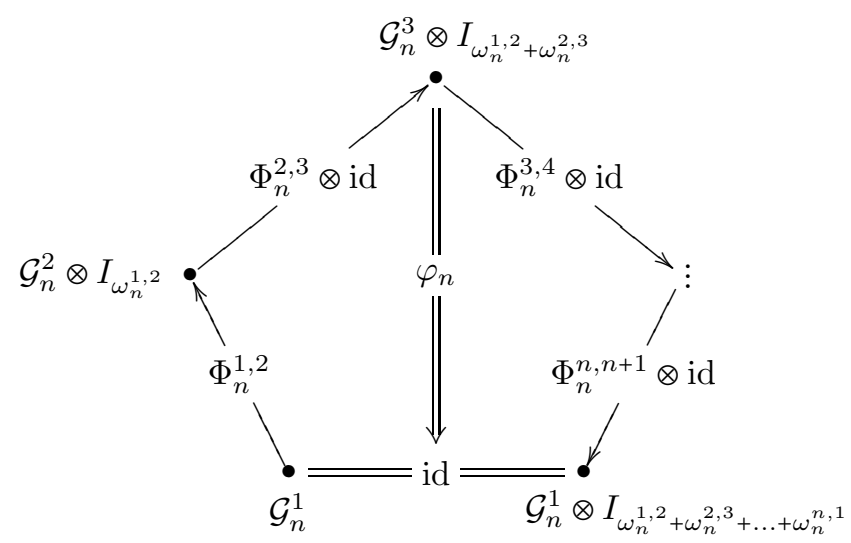

written in terms of 1-isomorphisms $\Phi_{n}^{k, k+1}=\left(\pi_{n}^{k, k+1}\right)^{*} \Phi^{\varepsilon_{n}^{k, k+1}}$ between gerbes $\mathcal{G}_{n}^{k}=\left(\pi_{n}^{k}\right)^{*} \mathcal{G}$, and the trivial gerbes with curvings $\omega_{n}^{k, k+1}=\varepsilon_{n}^{k, k+1}\left(\pi_{n}^{k, k+1}\right)^{*} \omega$. 
The manifold $T=\sqcup_{n \geq 3} T_{n}$, is called the $(\mathcal{G}, \mathcal{B})$-inter-bi-brane world-volume, and the manifold

$$
\mathscr{F}:=M \sqcup Q \sqcup T
$$

will be called the target space of background $\mathfrak{B}$. Also, in what follows, we shall often write the inter-bi-brane data in the form $\mathcal{J} \equiv\left(T_{n},\left(\varepsilon_{n}^{k, k+1}, \pi_{n}^{k, k+1}\right) ; \varphi_{n}\right)$ for brevity.

We give an important example of a complete string background in Section 5 .

Remark 2.2. The above definition invokes the structure of the 2-category $\mathfrak{B G} \mathfrak{b} \mathfrak{b} \nabla(\mathscr{F})$ of bundle gerbes with connection over the target space. The abstract 2-category was first introduced in Ref. Ste00] and further developed in Ref. Wal07b. Its 1-morphisms can be composed in the usual manner,

$$
\Phi_{2,3} \circ \Phi_{1,2}=\mathcal{G}_{1} \stackrel{\Phi_{1,2}}{\longrightarrow} \mathcal{G}_{2} \stackrel{\Phi_{2,3}}{\longrightarrow} \mathcal{G}_{3},
$$

whereas 2-isomorphisms can be composed in two different ways, namely horizontally

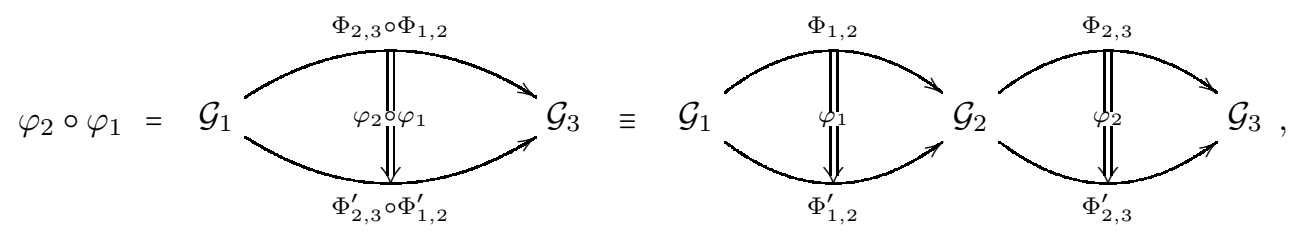

and vertically

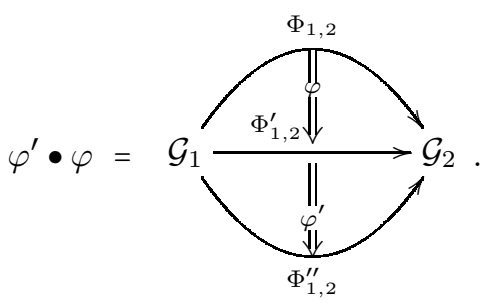

The two modes of composition are related by a coherence condition

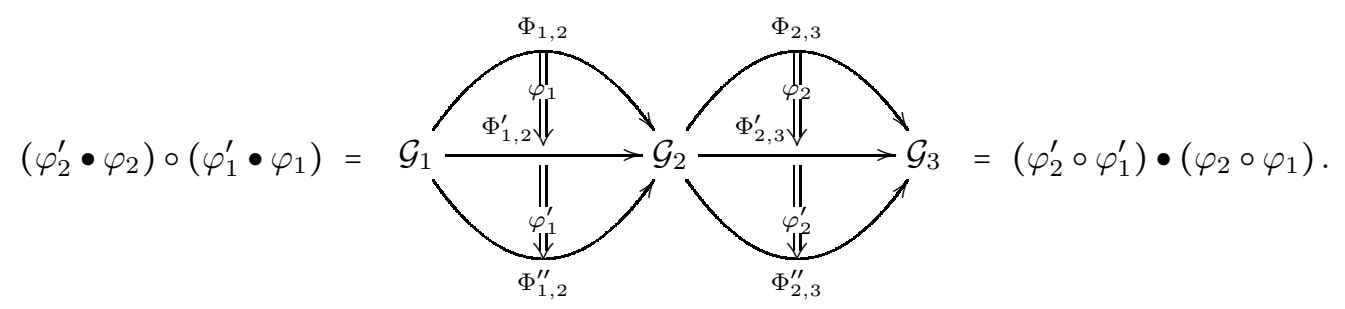

Furthermore, gerbes, 1-morphisms and 2-morphisms can be tensored and pulled back along smooth maps, both in a way consistent with the various compositions.

Remark 2.3. The bi-brane curvature satisfies the relations

$$
\begin{gathered}
\mathrm{d} \omega=-\Delta_{Q} \mathrm{H}, \\
\Delta_{T_{n}} \omega=0,
\end{gathered}
$$

where we have introduced the shorthand notation

$$
\Delta_{Q}=\iota_{2}^{*}-\iota_{1}^{*}, \quad \Delta_{T_{n}}=\sum_{k=1}^{n} \varepsilon_{n}^{k, k+1} \pi_{n}^{k, k+1 *}
$$

for the linear combinations of pullbacks that are going to appear frequently in the present paper. The two pullback operators satisfy the identity

$$
\Delta_{T_{n}} \circ \Delta_{Q}=0 .
$$

The target space of the string background is the codomain of the dynamical fields of the theory that we want to study, hence its name. This statement is rendered precise by 
Definition 2.4. Let $(\Sigma, \gamma)$ be a closed oriented Riemann surface, termed the world-sheet, and let $\Gamma$ be an oriented graph (possibly empty) embedded in $\Sigma$. We shall call it the defect quiver. We shall assume that, if not empty, $\Gamma$ is composed of oriented defect lines, closed or ending at points $\jmath$ called defect junctions of valence $\geq 3$, see Fig. 1. The connected components $\wp$ of $\Sigma \backslash \Gamma$ will be called patches. The set of patches will be denoted by $\mathfrak{P}_{\Sigma}$, the set of defect lines by $\mathfrak{E}_{\Gamma}$, and the set of defect junctions by $\mathfrak{V}_{\Gamma}$. Furthermore, let $\mathfrak{B}=(\mathcal{M}, \mathcal{B}, \mathcal{J})$ be a string background with target space $\mathscr{F}=M \sqcup Q \sqcup T$, as introduced in Definition 2.1. A network-field configuration $(\varphi \mid \Gamma)$ in string background $\mathfrak{B}$ on world-sheet $(\Sigma, \gamma)$ with defect quiver $\Gamma$ is a pair composed of the defect quiver $\Gamma$ embedded in the world-sheet $\Sigma$, together with a map

$$
\varphi: \Sigma \rightarrow \mathscr{F}
$$

such that

- $\varphi$ restricts to a smooth map $\Sigma \backslash \Gamma \rightarrow M$, a smooth map $\Gamma \backslash \mathfrak{V}_{\Gamma} \rightarrow Q$, and it sends $\mathfrak{V}_{\Gamma}$ into $T$ in such a manner that a defect junction $\jmath$ of valence $n_{\jmath}$ is mapped to $T_{n}$;

- for each (oriented) defect line $\ell, \varphi$ extends from the patch to the left (resp. right) of it (in the direction defined by the orientation of the line) to a smooth map $\varphi_{\mid 1}\left(\right.$ resp. $\left.\varphi_{\mid 2}\right): \ell \backslash \mathfrak{V}_{\Gamma} \rightarrow M$ with the property

$$
\varphi_{\mid \alpha}=\left.\iota_{\alpha} \circ \varphi\right|_{\ell \backslash \mathfrak{V}_{\Gamma}} ;
$$

- for $\jmath \in \mathfrak{V}_{\Gamma}$ an $n_{\jmath}$-valent defect junction and $\ell_{k, k+1}, k=1, \ldots, n$, the defect lines meeting at $\jmath$ and ordered in a cyclic way, the maps $\left.\varphi\right|_{\ell_{k, k+1} \backslash \mathfrak{V}_{\Gamma}}$ into $Q$ admit smooth extensions $\varphi_{k, k+1}$ to $\jmath$ such that

$$
\varphi_{k, k+1}(\jmath)=\pi_{n \jmath}^{k, k+1} \circ \varphi(\jmath) ;
$$

- for $\left(\jmath, \ell_{k, k+1}\right)$ as above, the orientation map takes the value $\varepsilon_{n_{\jmath}}^{k, k+1}(\varphi(\jmath))=+1$ if $\ell_{k, k+1}$ is oriented towards $\jmath$ and $\varepsilon_{n_{\jmath}}^{k, k+1}(\varphi(\jmath))=-1$ if the line is oriented away from $\jmath$ for vertices with defect line ordered counter-clockwise and the opposite values for vertices with defect lines ordered clockwise.

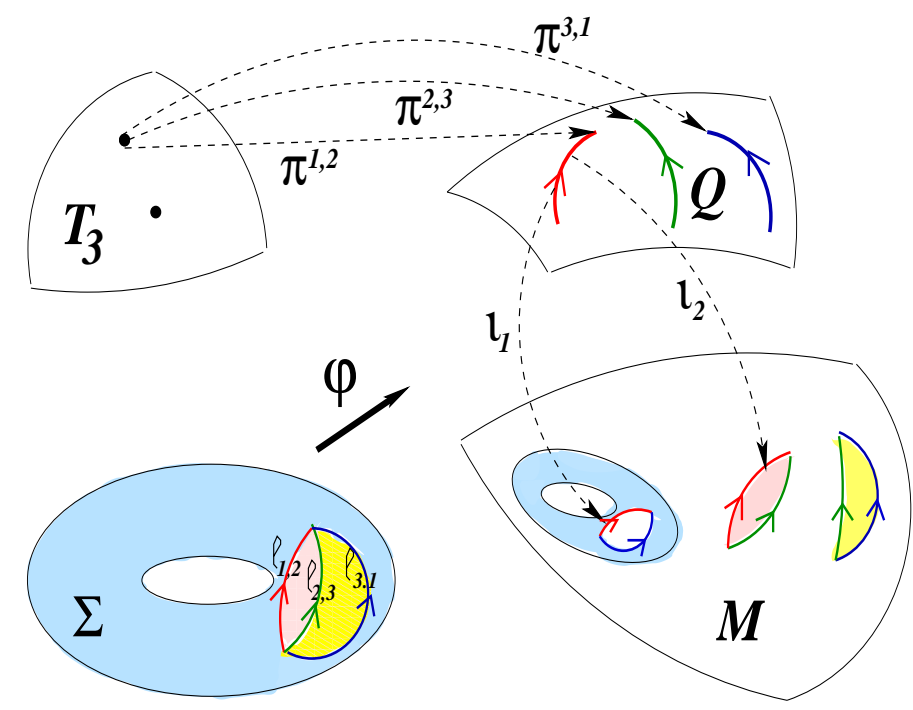

FIGURE 1. A Riemann surface with a defect quiver, and a network-field configuration.

Remark 2.5. It is to be stressed that the definition of a bi-brane encompasses the more familiar concept of a (D-)brane, assigned to the boundary of the world-sheet, cf. Ref. RS09. Indeed, upon taking the target space in the special form $M^{\prime}:=M \sqcup\{\bullet\}$ for an arbitrary singleton $\{\bullet\}$, alongside any manifold $Q$ equipped with a pair of smooth maps $\iota_{1}: Q \rightarrow$ $M \subset M^{\prime}$ and $\iota_{2}: Q \rightarrow\{\bullet\} \subset M^{\prime}$ (the constant map) as a bi-brane world-volume, we 
readily recover the standard notion in a new guise: The patch on one side of the defect line carrying the data of the (boundary) bi-brane is mapped to a single point, thereby mimicking an interface between a nontrivial phase of the $\sigma$-model and the trivial (void) $\sigma$-model.

We are now fully equipped to introduce the probability amplitudes of a two-dimensional non-linear $\sigma$-model whose symmetries will be studied in the remainder of the paper.

Definition 2.6. Let $(\varphi \mid \Gamma)$ be a network-field configuration in string background $\mathfrak{B}=$ $(\mathcal{M}, \mathcal{B}, \mathcal{J})$ on world-sheet $(\Sigma, \gamma)$ with defect quiver $\Gamma$, as introduced in Definition 2.4. The euclidean Feynman amplitude of $(\varphi \mid \Gamma)$ is

$$
\mathscr{A}[(\varphi \mid \Gamma) ; \gamma]:=\exp \left[-\frac{1}{2} \int_{\Sigma} \mathrm{g}\left(\mathrm{d} \varphi \wedge^{\star_{\gamma}} \mathrm{d} \varphi\right)\right] \operatorname{Hol}_{\mathcal{G}, \Phi,\left(\varphi_{n}\right)}(\varphi \mid \Gamma),
$$

in which

- $\mathrm{d} \varphi(\sigma)=\left.\partial_{u} \varphi^{\mu}(\sigma) \mathrm{d} \sigma^{u} \otimes \partial_{\mu}\right|_{\varphi(\sigma)}$ in local coordinates $\left\{\sigma^{u}\right\}$ on $\Sigma$ and $\left\{\varphi^{\mu}\right\}$ on $M$, and the target-space metric is assumed to act on the second factor of the tensor product;

- $\star_{\gamma}$ is the world-sheet Hodge operator determined by the world-sheet metric $\gamma$;

- the so-called 'topological', or Wess-Zumino contribution to the Feynman amplitude

$$
\mathscr{A}_{\mathrm{WZ}}[(\varphi \mid \Gamma)]:=\operatorname{Hol}_{\mathcal{G}, \Phi,\left(\varphi_{n}\right)}(\varphi \mid \Gamma)
$$

is given by the decorated-surface holonomy $\operatorname{Hol}_{\mathcal{G}, \Phi,\left(\varphi_{n}\right)}(\varphi \mid \Gamma)$ for the network-field configuration $(\varphi \mid \Gamma)$, described in detail in Ref. RS09 and generalising the holonomy of Ref. [FSW08 to the case with defect networks.

Remark 2.7. The quantum euclidean non-linear $\sigma$-model with defects is obtained by functional integration of probability amplitudes (2.8) over the network-field configurations $(\varphi \mid \Gamma)$.

In order to understand the fundamental property of the decorated-surface holonomy which is its invariance under transformations of the background realised by 1- and 2-isomorphisms and accompanied by compensating transformations of the bi-brane 1-isomorphism and of the inter-bi-brane 2-isomorphism, respectively, we shall invoke several basic properties of the

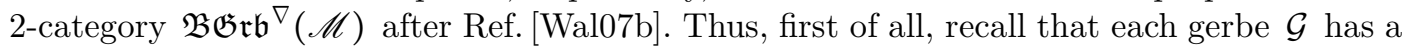
dual, to be denoted as $\mathcal{G}^{\vee}$, with the opposite curvature. Furthermore, every 1-isomorphism $\Psi: \mathcal{G}_{1} \stackrel{\cong}{\rightarrow} \mathcal{G}_{2}$ between gerbes $\mathcal{G}_{\alpha}, \alpha=1,2$, admits an inverse, $\Psi^{-1}: \mathcal{G}_{2} \stackrel{\cong}{\rightarrow} \mathcal{G}_{1}$, defined in such a manner that

$$
\left(\Psi_{1} \circ \Psi_{2}\right)^{-1}=\Psi_{2}^{-1} \circ \Psi_{1}^{-1},
$$

and there exist 2-isomorphisms

$$
d_{\Psi}: \Psi^{-1} \circ \Psi \stackrel{\cong}{=} \operatorname{id}_{\mathcal{G}_{1}}, \quad b_{\Psi}: \operatorname{id}_{\mathcal{G}_{2}} \stackrel{\cong}{=} \Psi \circ \Psi^{-1},
$$

called the 'death' and 'birth' 2-isomorphism, respectively. These satisfy the identities

$$
\lambda_{\Psi^{-1}}=\rho_{\Psi^{-1}} \bullet\left(d_{\Psi} \circ \operatorname{id}_{\Psi^{-1}}\right) \bullet\left(\operatorname{id}_{\Psi} \circ b_{\Psi}\right), \quad \rho_{\Psi}=\lambda_{\Psi} \bullet\left(\operatorname{id}_{\Psi} \circ d_{\Psi}\right) \bullet\left(b_{\Psi} \circ \operatorname{id}_{\Psi}\right),
$$

written in terms of the natural 2-isomorphisms

$$
\lambda_{\Psi}: \Psi \circ \operatorname{id}_{\mathcal{G}_{1}} \stackrel{\cong}{=} \Psi, \quad \rho_{\Psi}: \operatorname{id}_{\mathcal{G}_{2}} \circ \Psi \stackrel{\cong}{=} \Psi .
$$

They can be used to explicitly construct, for any 2-isomorphism $\psi: \Psi_{1} \stackrel{\cong}{=} \Psi_{2}$ between 1-isomorphisms $\Psi_{\alpha}, \alpha=1,2$, the associated 2-isomorphism $\psi^{\sharp}: \Psi_{2}^{-1} \stackrel{\cong}{\Longrightarrow} \Psi_{1}^{-1}$. Furthermore, there exists a canonical 2-isomorphism $i_{\Psi}:\left(\Psi^{-1}\right)^{-1} \stackrel{\cong}{\Longrightarrow} \Psi$. So far, we have pedantically kept the labels on the identity morphisms. For the sake of brevity, we shall drop these labels from now onwards, leaving it to the reader to reconstruct them from the context. We complete the summary of useful facts concerning the 2-category $\mathfrak{B G r b}^{\nabla}(\mathscr{M})$ by giving the following

Proposition 2.8. Gaw88, CJM02, Wal07a Let $\mathscr{M}$ be a smooth manifold.

(1) Given two gerbes $\mathcal{G}_{\alpha}, \alpha=1,2$, over $\mathscr{M}$ of the same curvature, there exists a flat gerbe $\mathcal{D}$ and a canonical 1-isomorphism

$$
\mathcal{G}_{2} \stackrel{\cong}{\rightarrow} \mathcal{D} \otimes \mathcal{G}_{1} .
$$

The two gerbes are 1-isomorphic iff $\mathcal{D} \cong I_{0}$. 
(2) Given two 1-isomorphisms $\Psi_{\alpha}: \mathcal{G} \stackrel{\cong}{\rightarrow} \mathcal{H}, \alpha=1,2$, between two gerbes $\mathcal{G}$ and $\mathcal{H}$ over $\mathscr{M}$, there exists a flat line bundle $D \rightarrow \mathscr{M}$ and a canonical 2-isomorphism

$$
\Psi_{2} \stackrel{\cong}{\Longrightarrow} D \otimes \Psi_{1},
$$

where on the right-hand side $D$ is viewed as a 1-isomorphism of the trivial gerbe $I_{0}$ using the canonical equivalence of categories $\operatorname{Bun}: \mathfrak{E n d}\left(I_{0}\right) \stackrel{\equiv}{\rightarrow} \mathfrak{B u n} \mathfrak{n}_{0}^{\nabla}(\mathscr{M})$ (for $\mathfrak{E n d}\left(I_{0}\right)$ the Hom-category of endomorphisms of the trivial gerbe $I_{0}$ and $\mathfrak{B u n} \mathfrak{n}_{0}^{\nabla}(\mathscr{M})$ the category of vector bundles with a traceless curvature). The two 1-isomorphisms are 2-isomorphic iff $D \cong J_{0}$ for $J_{0}$ the trivial line bundle with the trivial connection.

(3) Given two 2-isomorphisms $\psi_{\alpha}: \Psi \stackrel{\cong}{\Longrightarrow} \Xi, \alpha=1,2$, of two 1-isomorphisms $\Psi$ and $\Xi$ between a (common) pair of gerbes over $\mathscr{M}$, there exists a locally constant map $d \in C^{\infty}\left(\pi_{0}(\mathscr{M}), \mathrm{U}(1)\right)$ (for $\pi_{0}(\mathscr{M})$ the set of connected components of $\mathscr{M}$ ) such that

$$
\psi_{2}=d \otimes \psi_{1} .
$$

We are now ready to formulate

Proposition 2.9. RS09, Sec. 2.7] Let $\mathfrak{B}_{\alpha}=\left(\mathcal{M}_{\alpha}, \mathcal{B}_{\alpha}, \mathcal{J}_{\alpha}\right), \alpha=1,2$, be a pair of string backgrounds as in Definition 2.1, differing in the choice of the structure $\left(\mathcal{G}_{\alpha}, \Phi_{\alpha}, \varphi_{\alpha n} \mid n \geq 3\right)$ exclusively. Assume the existence of a 1-isomorphism

$$
\Psi: \mathcal{G}_{1} \stackrel{\cong}{\rightrightarrows} \mathcal{G}_{2},
$$

and of a 2-isomorphism

$$
\psi: \Phi_{1} \stackrel{\cong}{\Longrightarrow}\left(\iota_{2}^{*} \Psi^{-1} \otimes \mathrm{id}\right) \circ \Phi_{2} \circ \iota_{1}^{*} \Psi
$$

satisfying the equality

$$
\begin{aligned}
\varphi_{1 n}= & \left(d_{\Psi}\right)_{n}^{1} \bullet\left(\operatorname{id} \circ\left(i_{\Psi}^{\varepsilon_{n}^{1,2}}\right)_{n}^{1}\right) \\
& \bullet\left(\operatorname{id} \circ \lambda_{\Psi_{n}^{1}}\right) \bullet\left(\operatorname{id} \circ \varphi_{2 n} \circ \mathrm{id}\right) \bullet\left(\operatorname{id} \circ \lambda_{\Phi_{2 n}^{n, 1} \otimes \mathrm{id}} \circ \lambda_{\Phi_{2 n}^{n-1, n} \otimes \mathrm{id}} \circ \cdots \circ \lambda_{\Phi_{2 n}^{1,2} \otimes \mathrm{id}} \circ \mathrm{id}\right) \\
& \bullet\left(\operatorname{id} \circ\left(b_{\Psi}^{-1}\right)_{n}^{n} \circ \mathrm{id} \circ\left(b_{\Psi}^{-1}\right)_{n}^{n-1} \circ \mathrm{id} \circ \cdots \circ \mathrm{id} \circ\left(b_{\Psi}^{-1}\right)_{n}^{2} \circ \mathrm{id}\right) \\
& \bullet\left(\operatorname{id} \circ\left(i_{\Psi}^{\varepsilon_{n}^{n, 1}}\right)_{n}^{n} \circ \mathrm{id} \circ\left(i_{\Psi}^{\varepsilon_{n}^{n-1, n}}\right)_{n}^{n-1} \circ \mathrm{id} \circ \cdots \circ \mathrm{id} \circ\left(i_{\Psi}^{\varepsilon_{n}^{2,3}}\right)_{n}^{2} \circ \mathrm{id}\right) \\
& \bullet\left(\left(\psi_{n}^{n, 1} \otimes \mathrm{id}\right) \circ\left(\psi_{n}^{n-1, n} \otimes \mathrm{id}\right) \circ \cdots \circ \psi_{n}^{1,2}\right),
\end{aligned}
$$

the latter being written in terms of the 1-isomorphism

$$
\Psi_{n}^{1}:=\pi_{n}^{1 *} \Psi,
$$

and of the 2-isomorphisms

$$
\left(b_{\Psi}^{-1}\right)_{n}^{k}:=\pi_{n}^{k *} b_{\Psi}^{-1}, \quad\left(d_{\Psi}\right)_{n}^{1}:=\pi_{n}^{1 *} d_{\Psi},
$$

as well as

$$
\left(i_{\Psi}^{\varepsilon_{n}^{k, k+1}}\right)_{n}^{k}:=\pi_{n}^{k *} i_{\Psi}^{\varepsilon_{n}^{k, k+1}}, \quad \quad i_{\Psi}^{\varepsilon_{n}^{k, k+1}}:=\left\{\begin{array}{l}
\mathrm{id}_{\Psi} \quad \text { if } \\
i_{\Psi} \text { otherwise }
\end{array} \varepsilon_{n}^{k, k+1}=1,\right.
$$

and

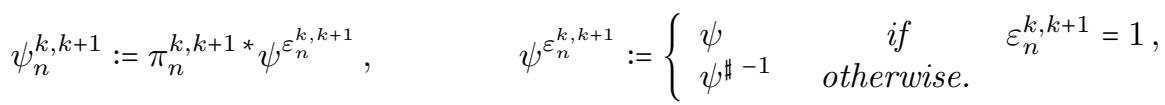

Then,

$$
\operatorname{Hol}_{\mathcal{G}_{2}, \Phi_{2},\left(\varphi_{2 n}\right)}(\varphi \mid \Gamma)=\operatorname{Hol}_{\mathcal{G}_{1}, \Phi_{1},\left(\varphi_{1 n}\right)}(\varphi \mid \Gamma) .
$$

The last proposition will play a central rôle in our discussion of the global gauge anomalies in the background of topologically trivial gauge fields. 
2.2. G-actions via simplicial G-spaces. Equivariance properties of the differential-geometric structures on $\mathscr{F}$ with respect to the group action may be conveniently described using categorial concepts such as the action groupoid $\mathrm{G} \ltimes \mathscr{F}$ and the nerve of the small category $\mathrm{G} \ltimes \mathscr{F}$. We begin by recalling a few basic concepts.

Definition 2.10. Let $\mathscr{M}$ be a smooth manifold equipped with a smooth (left) action of a Lie group $\mathrm{G}$

$$
\mathscr{M}_{\ell}: G \times \mathscr{M} \rightarrow \mathscr{M}:(g, m) \mapsto g \cdot m \equiv{ }^{\mathscr{M}_{g}}(m) .
$$

Such a manifold will be referred to as a G-space from now onwards. Let $\mathfrak{g}$ be the Lie algebra of G. To every element $X \in \mathfrak{g}$, we associate a vector field $\bar{X}$ on $\mathscr{M}$ acting on smooth functions according to the formula

$$
(\bar{X} f)(m)=\left.\frac{\mathrm{d}}{\mathrm{d} t}\right|_{t=0} f\left(\mathrm{e}^{-t X} \cdot m\right) .
$$

In order to keep subsequent equations more transparent, we set up

Convention 2.11. Let $\mathscr{M}$ be a G-space and let $\left\{\mathscr{M} \mathscr{K}_{a}\right\}_{a=1,2, \ldots, \operatorname{dim} g}$ be the fundamental vector fields on $\mathscr{M}$ associated, through Eq. (2.11), to the generators

$$
t_{a}, a=1,2, \ldots, \operatorname{dim} \mathfrak{g}, \quad\left[t_{a}, t_{b}\right]=f_{a b c} t_{c}
$$

of the Lie algebra $\mathfrak{g}$, with the corresponding Lie bracket

$$
\left[{ }^{\mathscr{M}} \mathscr{K}_{a},{ }^{\mathscr{M}} \mathscr{K}_{b}\right]=f_{a b c} \mathscr{M}^{\mathscr{K}} \mathscr{K}_{c} \text {. }
$$

Contraction with $\mathscr{M} \mathscr{K}_{a}$ will be denoted as

$$
\imath_{a}:=\imath \varkappa_{\mathscr{M}},
$$

and the corresponding Lie derivative as

$$
\mathscr{L}_{a}:=\mathscr{L}_{\mathscr{M}_{\mathscr{K}_{a}}} .
$$

Furthermore, given a $\mathfrak{g}$-valued 1-form $\eta=\eta^{a} \otimes t_{a} \in \Omega^{1}(\mathscr{M}) \otimes \mathfrak{g}$ on $\mathscr{M}$, and a $p$-form $\xi \in \Omega^{p}(\mathscr{M})$, we write

$$
\bar{\eta}:=\eta^{a} \otimes \mathscr{M}^{\mathscr{K}}
$$

and define

$$
\bar{\eta} \cdot \xi:=\eta^{a} \wedge\left(\imath_{a} \xi\right) .
$$

This formula gives an obvious meaning to symbols such as $\mathrm{e}^{\bar{\eta}} \cdot \xi$.

The following definition fixes the standard terminology used throughout this paper.

Definition 2.12. A smooth $p$-form $\eta \in \Omega^{p}(\mathscr{M})$ will be called $\mathfrak{g}$-horizontal iff it is annihilated by contraction with all the vector fields $\mathscr{M}_{K_{a}}$,

$$
\imath_{a} \eta=0 .
$$

It will be termed $\mathfrak{g}$-invariant resp. G-invariant iff it satisfies the conditions

$$
\mathscr{L}_{a} \eta=0
$$

resp.

$$
\mathscr{M}_{g}^{*} \eta=\eta, \quad g \in \mathrm{G} .
$$

A $p$-form which is both $\mathfrak{g}$-horizontal and $\mathfrak{g}$-invariant resp. G-invariant will be called $\mathfrak{g}$-basic resp. G-basic.

Next, let $\zeta=\zeta_{a} \otimes \tau^{a} \in \Omega^{p}(\mathscr{M}) \otimes \mathfrak{g}^{*}$ be a smooth $\mathfrak{g}^{*}$-valued $p$-form on $\mathscr{M}$, written in terms of the basis $\left\{\tau^{a}\right\}$ of $\mathfrak{g}^{*}$ dual to $\left\{t_{a}\right\}$. For $X \in \mathfrak{g}$, we shall write

$$
\zeta(X):=\tau^{a}(X) \zeta_{a} .
$$

A $p$-form $\eta$ will be called $\mathfrak{g}$-equivariant resp. G-equivariant iff it satisfies the condition

$$
\mathscr{L}_{a} \zeta_{b}=f_{a b c} \zeta_{c}
$$

resp.

$$
\left({ }^{\mathscr{M}} \ell_{g}^{*} \zeta\right)(X)=\zeta\left(\operatorname{Ad}_{g^{-1}} X\right)
$$


for all $X \in \mathfrak{g}$.

We may now introduce

Definition 2.13. Let $\mathscr{M}$ be a G-space, with the (left) action of the group G given by Eq. (2.10). The action groupoid

$$
\mathrm{G} \ltimes \mathscr{M}: \mathrm{G} \times \mathscr{M} \underset{t}{\stackrel{s}{\rightleftarrows}} \mathscr{M}
$$

is a (small) category with the object and morphism sets

$$
\mathrm{Ob}(\mathrm{G} \ltimes \mathscr{M})=\mathscr{M}, \quad \operatorname{Mor}(\mathrm{G} \ltimes \mathscr{M})=\mathrm{G} \times \mathscr{M},
$$

with the source and target maps

$$
s(g, x)=x, \quad t(g, x)=g \cdot x,
$$

with the identity morphisms

$$
\operatorname{id}_{x}=(e, x)
$$

( $e$ is the group unit), and, finally, with the composition of morphisms

$$
(g, h \cdot x) \circ(h, x)=(g \cdot h, x) .
$$

Both $\mathrm{G} \times \mathscr{M}$ and $\mathscr{M}$ carry the structure of a G-space, the two being related by

Proposition 2.14. Let $\mathrm{G} \ltimes \mathscr{M}$ be the action groupoid over a $\mathrm{G}$-space $\mathscr{M}$ equipped with a (left) G-action (2.10), and let $\mathscr{M}_{a}, a=1,2, \ldots, \operatorname{dim} \mathfrak{g}$ be the corresponding fundamental vector fields on $\mathscr{M}$, introduced in Definition 2.10. Denote by $L_{a}$ (resp. $R_{a}$ ) the left-invariant (resp. right-invariant) vector fields on $\mathrm{G}$ dual to the left-invariant (resp. right-invariant) MaurerCartan 1-forms $\theta_{L}^{a}$, with $\theta_{L}(g)=g^{-1} \mathrm{~d} g=\theta_{L}^{a}(g) \otimes t_{a} \quad$ (resp. $\theta_{R}^{a}$, with $\theta_{R}(g)=\mathrm{d} g g^{-1}=$ $\left.\theta_{R}^{a}(g) \otimes t_{a}\right)$. The source and target maps of $\mathrm{G} \ltimes \mathscr{M}$ intertwine the left action (2.10) of $\mathrm{G}$ on $\mathscr{M}$ with the combined adjoint and left action of $\mathrm{G}$ on $\mathrm{G} \times \mathscr{M}$,

$$
s\left(\operatorname{Ad}_{h}(g), h . x\right)=h . s(g, x), \quad t\left(\operatorname{Ad}_{h}(g), h . x\right)=h . t(g, x) .
$$

In particular, the vector field $\mathscr{M}^{K_{a}^{(0)}} \equiv \mathscr{M} \mathscr{K}_{a}$ on $M$ can be regarded as the pushforward of the vector field

$$
\mathscr{M}^{K_{a}^{(1)}}(g, x)=\left(L_{a}-R_{a}\right)(g)+{ }^{\mathscr{M}} \mathscr{K}_{a}(x) .
$$

Proof. Obvious, through inspection.

In the next step, we invoke some elementary notions of simplicial analysis that will prove useful in what follows. The interested reader is urged to consult, e.g., Ref. Wei94 for further details. The point of departure is

Definition 2.15. The category $\Delta$ has subsets $[m]:=\{0,1, \ldots, m\}$ of the set of nonnegative integers as its objects, and order-preserving maps

$$
\theta:[l] \rightarrow[m]: i \mapsto \theta(i), \quad i<j \Rightarrow \theta(i)<\theta(j)
$$

as its morphisms. We shall use the notation $\operatorname{Hom}_{\Delta}([l],[m])=: \Delta(l, m)$.

We have

Proposition 2.16. The Hom-sets of the category $\Delta$ have the following properties:

(i) $l>m \Rightarrow \Delta(l, m)=\varnothing$;

(ii) $\Delta(m, m)=\left\{\operatorname{id}_{[m]}\right\}$;

(iii) $\Delta(m-1, m)=\left\{\theta_{k}^{(m)} \mid k=0,1, \ldots, m\right\}$, with the universal coface maps $\theta_{k}^{(m)}(i)=\left\{\begin{array}{lc}i & \text { if } \\ i+1 & \text { otherwise }\end{array}\right.$, satisfying the cosimplicial identities

$$
\theta_{k}^{(m+1)} \circ \theta_{l}^{(m)}=\theta_{l}^{(m+1)} \circ \theta_{k-1}^{(m)}
$$

for $0 \leq l<k$ and $k=0,1, \ldots, m+1$. 
Proof. Obvious, through direct inspection.

Next, we introduce

Definition 2.17. An incomplete simplicial set is a family $\left\{S^{m}\right\}_{m=0,1 \ldots \text { of sets, indexed }}$ by non-negative integers, together with a collection of maps $\sigma_{k}^{(m+1)}: S^{m+1} \rightarrow S^{m}, k=$ $0,1, \ldots, m+1$ between them, termed the face maps, that satisfy the simplicial identities

$$
\sigma_{l}^{(m)} \circ \sigma_{k}^{(m+1)}=\sigma_{k-1}^{(m)} \circ \sigma_{l}^{(m+1)}
$$

for all $0 \leq l<k$ and $k=0,1, \ldots, m+1$.

Upon employing the straightforward one-to-one correspondence between incomplete simplicial manifolds, i.e. incomplete simplicial sets internal to the subcategory Man of smooth manifolds, and contravariant functors $M: \Delta \rightarrow$ Man (cf. GW09), we arrive at

Definition 2.18. The nerve

$$
\cdots \mathrm{G}^{2} \times \mathscr{M} \equiv \mathrm{G} \times \mathscr{M} \Longrightarrow \mathscr{M}
$$

of the action groupoid $\mathrm{G} \ltimes \mathscr{M}$ is a contravariant functor G $\mathscr{M}: \Delta \rightarrow$ G-Man from $\Delta$ to the category G-Man of G-spaces, with the object component

$$
\text { G } \mathscr{M}([0]):=\mathscr{M}, \quad \text { G } \mathscr{M}([m]):=\mathrm{G}^{m} \times \mathscr{M}, \quad m \geq 1,
$$

and the morphism component determined by its restriction to the universal coface maps,

$$
\mathrm{G} \mathscr{M}\left(\theta_{k}^{(m)}\right):=\mathscr{M} d_{k}^{(m)},
$$

with the resultant face maps of the nerve, ${ }^{\mathscr{M}} d_{k}^{(m)}: \mathrm{G}^{m} \times \mathscr{M} \rightarrow \mathrm{G}^{m-1} \times \mathscr{M}$ (represented by the arrows in the diagram), given explicitly in the form

$$
\begin{aligned}
\mathscr{M}_{0}^{(1)}(g, x) & =x, \\
\mathscr{M}_{1}^{(1)}(g, x) & =g \cdot x, \\
\mathscr{M} d_{0}^{(m)}\left(g_{m}, g_{m-1}, \ldots, g_{1}, x\right) & =\left(g_{m-1}, g_{m-2}, \ldots, g_{1}, x\right), \\
\mathscr{M}_{m}^{(m)}\left(g_{m}, g_{m-1}, \ldots, g_{1}, x\right) & =\left(g_{m}, g_{m-1}, \ldots, g_{2}, g_{1} \cdot x\right), \\
\mathscr{M}_{i}^{(m)}\left(g_{m}, g_{m-1}, \ldots, g_{1}, x\right) & =\left(g_{m}, g_{m-1}, \ldots, g_{m+2-i}, g_{m+1-i} \cdot g_{m-i}, g_{m-1-i}, \ldots, g_{1}, x\right) .
\end{aligned}
$$

The nerve G $\mathscr{M}$ is additionally endowed with the degeneracy maps $\mathscr{M}_{k}^{(m)}: \mathrm{G}^{m} \times \mathscr{M} \rightarrow$ $\mathrm{G}^{m+1} \times \mathscr{M}$ defined as

$$
\begin{aligned}
\mathscr{M}_{0}^{(0)}(x) & =(e, x), \\
\mathscr{M}_{i}^{(m)}\left(g_{m}, g_{m-1}, \ldots, g_{1}, x\right) & =\left(g_{m}, g_{m-1}, \ldots, g_{m+1-i}, e, g_{m-i}, g_{m-1-i}, \ldots, g_{1}, x\right) .
\end{aligned}
$$

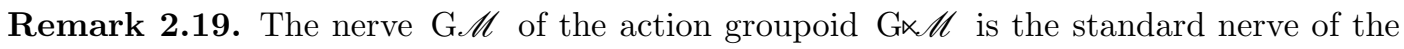

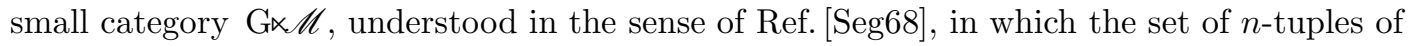
composable morphisms in $\mathrm{G} \ltimes \mathscr{M}$ has been identified with $\mathrm{G}^{m} \times \mathscr{M}$ in a natural manner,

$$
\begin{array}{r}
\left(\left(g_{m},\left(g_{m-1} \cdot g_{m-2} \cdots g_{1}\right) \cdot x\right),\left(g_{m-1},\left(g_{m-2} \cdot g_{m-3} \cdots g_{1}\right) \cdot x\right), \ldots,\left(g_{1}, x\right)\right) \\
\equiv\left(g_{m}, g_{m-1}, \ldots, g_{1}, x\right) .
\end{array}
$$

Note also that the face maps of G $\mathscr{M}$ satisfy the simplicial identities, including, in particular, the quadratic relations

$$
\mathscr{M} d_{i}^{(m)} \circ \mathscr{M} d_{j}^{(m+1)}={ }^{\mathscr{M}} d_{j-1}^{(m)} \circ \mathscr{M} d_{i}^{(m+1)} \quad \text { for } i<j .
$$


The face maps of G $\mathscr{M}$ are G-equivariant. The corresponding G-action on $\mathrm{G}^{m} \times \mathscr{M}$ is

$$
\begin{aligned}
\mathscr{M}_{\ell}(m): & \mathrm{G} \times\left(\mathrm{G}^{m} \times \mathscr{M}\right) \rightarrow \mathrm{G}^{m} \times \mathscr{M} \\
: & \left(h,\left(g_{m}, g_{m-1}, \ldots, g_{1}, x\right)\right) \mapsto\left(\operatorname{Ad}_{h}\left(g_{m}\right), \operatorname{Ad}_{h}\left(g_{m-1}\right), \ldots, \operatorname{Ad}_{h}\left(g_{1}\right), h . x\right),
\end{aligned}
$$

with $\mathscr{M}_{\ell}^{(0)} \equiv \mathscr{M}_{\ell}$. The action gives rise to vector fields on $\mathrm{G}^{n} \times \mathscr{M}$ of the form

$$
\mathscr{M}^{\mathscr{K}_{a}{ }^{(m)}}\left(g_{m}, g_{m-1}, \ldots, g_{1}, x\right)=\sum_{k=1}^{m}\left(L_{a}-R_{a}\right)\left(g_{k}\right)+{ }^{\mathscr{M}} \mathscr{K}_{a}(x) .
$$

The face maps can also be used to associate with G $\mathscr{M}$ (and for a choice of a differential sheaf over the nerve) a pullback cohomology in the sense of Ref. [Mur96].

Proposition 2.20. The pullback operators

$$
\mathscr{M}_{\mathrm{G}}^{(m)}=\sum_{i=0}^{m+1}(-1)^{m+1-i \mathscr{M}} d_{i}^{(m+1) *},
$$

square to zero,

$$
\mathscr{M} \delta_{\mathrm{G}}^{(m)} \circ{ }^{\mathscr{M}} \delta_{\mathrm{G}}^{(m-1)}=0
$$

Proof. A straightforward check.

The cohomology will be seen to encode the G-equivariance relations satisfied by elements of the description of the $\sigma$-model in the presence of defects.

Next, specialising our discussion to the $\sigma$-model context, we assume that the components $M, Q$ and $T_{n}$ of the target space are G-spaces, and that the various maps between them involved in the string background are G-maps, i.e. that they commute with the actions of G. We have the following straightforward

Proposition 2.21. Let $\mathfrak{B}=(\mathcal{M}, \mathcal{B}, \mathcal{J})$ be a string background as in Definition 2.1 such that the components $M, Q$ and $T_{n}$ of the target space $\mathscr{F}=M \sqcup Q \sqcup T$ are G-spaces. If the associated maps $\iota_{\alpha} \equiv \iota_{\alpha}^{(0)}, \alpha=1,2$ and $\pi_{n}^{k, k+1} \equiv \pi_{n}^{k, k+1(0)}, k=1,2, \ldots, n$, are G-maps, then they extend to natural transformations

$$
\mathrm{G} \iota_{\alpha}: \mathrm{G} Q \rightarrow \mathrm{G} M, \quad \mathrm{G} \pi_{n}^{k, k+1}: \mathrm{G} T_{n} \rightarrow \mathrm{G} Q
$$

between the respective nerve functors of Definition 2.18 by setting

$$
\begin{aligned}
\iota_{\alpha}^{(m)} & \equiv \mathrm{G}_{\alpha}([m]):=\mathrm{id}_{\mathrm{G}^{m}} \times \iota_{\alpha}: \mathrm{G}^{m} \times Q \rightarrow \mathrm{G}^{m} \times M, \\
\pi_{n}^{k, k+1(m)} & \equiv \mathrm{G} \pi_{n}^{k, k+1}([m]):=\mathrm{id}_{\mathrm{G}^{m}} \times \pi_{n}^{k, k+1}: \mathrm{G}^{m} \times T_{n} \rightarrow \mathrm{G}^{m} \times Q .
\end{aligned}
$$

The last proposition will prove useful in a cohomological classification of G-equivariant string backgrounds in Section 11. From now on, we suppose that the assumptions of Proposition 2.21 are satisfied.

The G-equivariant maps $\iota_{\alpha}^{(m)}$ give rise to operators

$$
\Delta_{Q}^{(m)}:=\iota_{2}^{(m) *}-\iota_{1}^{(m) *}, \quad \Delta_{T_{n}}^{(m)}:=\sum_{k=1}^{n} \varepsilon_{n}^{k, k+1} \pi_{n}^{k, k+1(m) *}
$$

extending $\Delta_{Q} \equiv \Delta_{Q}^{(0)}$ and $\Delta_{T_{n}} \equiv \Delta_{T_{n}}^{(0)}$, respectively, and satisfying the simple relations

$$
{ }^{Q} \delta_{\mathrm{G}}^{(m)} \circ \Delta_{Q}^{(m)}=\Delta_{Q}^{(m+1)} \circ{ }^{M} \delta_{\mathrm{G}}^{(m)}
$$

and

$$
{ }^{T_{n}} \delta_{\mathrm{G}}^{(m)} \circ \Delta_{T_{n}}^{(m)}=\Delta_{T_{n}}^{(m+1)} \circ{ }^{Q} \delta_{\mathrm{G}}^{(m)},
$$

to be exploited presently. 
Convention 2.22. In order to unclutter the notation, and, at the same time, avoid confusion with the various indexing schemes employed in the paper, we fix once and for all a convention for pullbacks of differential forms along canonical projection maps. Thus, for any $p$-form $\eta$ on a smooth space $\mathscr{M}:=\mathscr{M}_{1} \times \mathscr{M}_{2} \times \cdots \times \mathscr{M}_{N}$ equipped with canonical projections $\operatorname{pr}_{i_{1}, i_{2}, \ldots, i_{n}}$ : $\mathscr{M} \rightarrow \mathscr{M}_{i_{1}} \times \mathscr{M}_{i_{2}} \times \cdots \times \mathscr{M}_{i_{n}}$ given for $1 \leq i_{1}<i_{2}<\ldots<i_{n} \leq N$, we denote

$$
\eta_{\left[i_{1}, i_{2}, \ldots, i_{n}\right]^{*}}:=\operatorname{pr}_{i_{1}, i_{2}, \ldots, i_{n}}^{*} \eta \text {. }
$$

In particular,

$$
\eta_{i^{*}} \equiv \eta_{[i]^{*}}=\operatorname{pr}_{i}^{*} \eta
$$

for any $1 \leq i \leq N$. Analogous convention will be used for geometric objects such as bundles, gerbes etc.

2.3. Rigid symmetries of the $\sigma$-model. These are the symmetries induced by a group action on the target space $\mathscr{F}$. They transform fields of the model by composing them with the target-space transformations in a way that leaves the probability amplitudes unchanged. The study of continuous rigid symmetries entails, on the infinitesimal level, the analysis of variations of the Feynman amplitudes of Eq. (2.8) generated by the action of smooth vector fields on the target space. The following result will, therefore, prove instrumental.

Proposition 2.23. [RS09, App. A.2] Let $\mathscr{A}$ be the functional 2.8), and let $\mathscr{V}$ be a vector field on $\mathscr{F}$, with restrictions $\left.\mathscr{V}\right|_{M}={ }^{M \mathscr{V}},\left.\mathscr{V}\right|_{Q}={ }^{Q \mathscr{V}}$ and $\left.\mathscr{V}\right|_{T_{n}}={ }^{T_{n} \mathscr{V}}$, subject to the alignment conditions

$$
\iota_{\alpha *}{ }^{Q} \mathscr{V}=\left.{ }^{M} \mathscr{V}\right|_{\iota_{\alpha}(Q)}, \quad \quad \pi_{n *}^{k, k+1} T_{n} \mathscr{V}=\left.{ }^{Q} \mathscr{V}\right|_{\pi_{n}^{k, k+1}\left(T_{n}\right)} .
$$

Denote by $\xi_{t}: \mathscr{F} \rightarrow \mathscr{F}$ the (local) flow of $\mathscr{V}$ (assumed to exist). The variation of $\mathscr{A}$ along $\xi$ is then given by

$$
\begin{aligned}
& \left.\frac{\mathrm{d}}{\mathrm{d} t}\right|_{t=0} \mathscr{A}[(\varphi \mid \Gamma) ; \gamma] \\
= & \left(-\frac{1}{2} \int_{\Sigma}\left(\mathscr{L}_{\mathscr{V}} \mathrm{g}\right)_{\varphi}\left(\mathrm{d} \varphi{ }^{\wedge}{ }^{\star} \gamma \mathrm{d} \varphi\right)+\mathrm{i} \int_{\Sigma} \varphi^{*}\left(\imath_{\mathscr{V}} \mathrm{H}\right)+\mathrm{i} \int_{\Gamma} \varphi^{*}\left(\imath_{\mathscr{V}} \omega\right)\right) \mathscr{A}\left[\left(\xi_{t} \circ \varphi \mid \Gamma\right) ; \gamma\right] .
\end{aligned}
$$

Properties of vector fields whose flows preserve the Feynman amplitudes may be read off from Eq. 2.25).

Proposition 2.24. Let $\mathscr{F}^{\mathscr{K}} \mathscr{K}_{a}$ be the fundamental vector fields on $\mathscr{F}$, introduced in Definition 2.10 (their restrictions $\mathscr{M}_{a}, \mathscr{M}=M, Q, T_{n}$ satisfy the alignment conditions (2.24)). Then, the $\sigma$-model Feynman amplitudes of Eq. (2.8) are invariant under rigid translations of the $\sigma$-model field $\varphi: \Sigma \rightarrow \mathscr{F}$ along the flows of the vector fields $\mathscr{F}^{\mathscr{K}}$ on $\mathscr{F}$ iff the components ${ }^{M} \mathscr{K}_{a}$ are Killing for $\mathrm{g}$,

$$
\mathscr{L}_{a} \mathrm{~g}=0,
$$

and there exist a collection of 1-forms $\kappa_{a}$ on $M$ and a collection of functions $k_{a}$ on $Q$ such that

$$
\begin{aligned}
\imath_{a} \mathrm{H} & =-\mathrm{d} \kappa_{a}, \\
\imath_{a} \omega+\Delta_{Q} \kappa_{a} & =-\mathrm{d} k_{a}, \\
\Delta_{T_{n}} k_{a} & =0 .
\end{aligned}
$$

Proof. Using integration by parts, it is straightforward to see that conditions (2.26)-(2.29) are sufficient for the variation of the amplitudes in the direction of $\mathscr{F} \mathscr{K}_{a}$ to vanish. A proof of the claim that they are necessary is given in Appendix A.

Taken together, the fields $\mathscr{K}_{a}, \kappa_{a}$ and $k_{a}$ compose a section of a generalised tangent bundle over (the respective components of) the target space. On smooth sections of the latter, we may define a bracket operation generalising the Lie bracket of sections of the tangent bundle and closing on the linear span of sections generating rigid symmetries of the $\sigma$-model. It will be seen to play an important and natural rôle in our considerations of the constraints for 
a consistent gauging later on. In the meantime, let us be more specific about the algebraic structure on the generalised tangent bundle of the target space that emerges from our analysis.

Definition 2.25. Consider a smooth manifold $\mathscr{F}=M \sqcup Q \sqcup T$ whose components $M, Q$ and $T=\sqcup_{n \geq 3} T_{n}$ support smooth maps $\iota_{\alpha}: Q \rightarrow M, \alpha=1,2$ and $\pi_{n}^{k, k+1}: T_{n} \rightarrow Q, k=1,2, \ldots, n$, satisfying Eq. 2.1), as well as smooth forms: a closed $\mathrm{H} \in \Omega^{3}(M)$ and $\omega \in \Omega^{2}(Q)$ related to $\mathrm{H}$ as in Eq. (2.3) and satisfying Eq. (2.4). Denote by $\mathrm{E}^{(p, q)} \mathscr{M}:=\wedge^{p} \mathrm{~T} \mathscr{M} \oplus \wedge^{q} \mathrm{~T}^{*} \mathscr{M}$ the generalised tangent bundle of type $(p, q)$ over $\mathscr{M}=M, Q, T_{n}$, and introduce the total generalised tangent bundle

$$
\mathrm{E} \mathscr{F}:=\mathrm{E}^{(1,1)} M \sqcup \mathrm{E}^{(1,0)} Q \sqcup \mathrm{T} T .
$$

The $\left(\mathrm{H}, \omega ; \Delta_{Q}\right)$-twisted bracket structure on $\mathrm{E} \mathscr{F}$ is the quadruple

$$
\left.\left(\mathrm{E} \mathscr{F}, \llbracket \cdot, \cdot \rrbracket^{\left(\mathrm{H}, \omega ; \Delta_{Q}\right)},(\cdot, \cdot)\right\lrcorner, \alpha_{\mathrm{\top} \mathscr{F}}\right)=: \mathfrak{M}^{\left(\mathrm{H}, \omega ; \Delta_{Q}\right)} \mathscr{F}
$$

in which $\alpha_{\mathrm{T} \mathscr{F}}: \mathrm{E} \mathscr{F} \rightarrow \mathrm{T} \mathscr{F}$ is the canonical (component-wise) projection, $\left.(\cdot, \cdot)\right\lrcorner: \Gamma(\mathrm{E} \mathscr{F})^{2} \rightarrow$ $C^{\infty}(M, \mathbb{R})$ is the scalar product on the component $\mathrm{E}^{(1,1)} M$ of $\mathrm{E} \mathscr{F}$, given by

$$
(\mathscr{V} \oplus v, \mathscr{W} \oplus \varpi)\lrcorner:=\frac{1}{2}\left(\imath_{\mathscr{V}} \varpi+\imath_{\mathscr{W}} v\right), \quad \mathscr{V} \oplus v, \mathscr{W} \oplus \varpi \in \Gamma\left(\mathrm{E}^{(1,1)} M\right),
$$

and $\llbracket \cdot, \cdot \rrbracket^{\left(\mathrm{H}, \omega ; \Delta_{Q}\right)}$ is a bilinear antisymmetric operation on smooth sections of E $\mathscr{F}$, with restrictions

$$
\begin{aligned}
& \llbracket \mathfrak{V}_{1},\left.\mathfrak{V}_{2} \rrbracket^{\left(\mathrm{H}, \omega ; \Delta_{Q}\right)}\right|_{M}=\left[{ }^{M} \mathscr{V}_{1},{ }^{M_{\mathscr{V}}}\right] \oplus\left(\mathscr{L} \mathscr{V}_{1} v_{2}-\mathscr{L} \mathscr{V}_{2} v_{1}-\frac{1}{2} \mathrm{~d}\left(\imath \mathscr{V}_{1} v_{2}-\imath \mathscr{V}_{2} v_{1}\right)+\imath \mathscr{V}_{1} \imath \mathscr{V}_{2} \mathrm{H}\right), \\
& \llbracket \mathfrak{V}_{1},\left.\mathfrak{V}_{2} \rrbracket^{\left(\mathrm{H}, \omega ; \Delta_{Q}\right)}\right|_{Q}=\left[\mathscr{V}_{1},{ }_{\mathscr{V}}\right] \oplus\left(\mathscr{L} \mathscr{V}_{1} f_{2}-\mathscr{L}_{\mathscr{V}_{2}} f_{1}+\imath \mathscr{V}_{1} \imath \mathscr{V}_{2} \omega+\frac{1}{2}\left(\imath \mathscr{V}_{1} \Delta_{Q} v_{2}-\imath \mathscr{V}_{2} \Delta_{Q} v_{1}\right)\right), \\
& \llbracket \mathfrak{V}_{1},\left.\mathfrak{V}_{2} \rrbracket^{\left(\mathrm{H}, \omega ; \Delta_{Q}\right)}\right|_{T_{n}}=\left[{ }^{Q_{n}} \mathscr{V}_{1},{ }^{T_{n}} \mathscr{V}_{2}\right],
\end{aligned}
$$

expressed in terms of the restrictions $\left.\mathfrak{V}_{i}\right|_{M}={ }^{M \mathscr{V}_{i}} \oplus v_{i},\left.\mathfrak{V}_{i}\right|_{Q}=\mathscr{Q \mathscr { V }}_{i} \oplus f_{i}$ and $\left.\mathfrak{V}_{i}\right|_{T_{n}}={ }^{T_{n}} \mathscr{V}_{i}$ of $\mathfrak{V}_{i} \in \Gamma(\mathrm{E} \mathscr{F}), i=1,2$.

Remark 2.26. It is to be noted that the restriction of the $\left(\mathrm{H}, \omega ; \Delta_{Q}\right)$-twisted bracket structure to $\mathrm{E}^{(1,1)} M$ defines the familiar Courant algebroid of Refs. CCou90, Dor93, LWX98, twisted by the gerbe curvature $\mathrm{H}$ in the manner first discussed in Ref. [SW01]. Its rôle in the context of $\sigma$-model symmetries was observed already in Ref. AS05, and an intrinsic gerbetheoretic interpretation (in terms of generalised tangent bundles twisted by local data of a gerbe) was put forward in Ref. Hit03]. A full-fledged canonical interpretation of the complete algebraic structure on the generalised tangent bundle of the target space alongside a unified description in the language of the 2-category of bundle gerbes with curving and connection over $\mathscr{F}$ is given in Ref. Sus12]. Below, we invoke some of these results.

Proposition 2.27. Sus12, Prop. 5.5] In the notation of Definition 2.25, denote by $\Gamma_{\sigma}(\mathrm{E} \mathscr{F})$ the subspace of all smooth sections $\mathfrak{V}=\left({ }^{M \mathscr{V}} \oplus v,{ }^{Q \mathscr{V}} \oplus f,{ }^{T_{n} \mathscr{V}}\right)$ of $\mathrm{E} \mathscr{F}$, to be termed $\sigma$ symmetric, that satisfy the alignment conditions (2.24) and obey

$$
\mathscr{L}_{\mathscr{V}} \mathrm{g}=0, \quad\left\{\begin{array}{l}
\imath_{\mathscr{V}} \mathrm{H}=-\mathrm{d} v \\
\imath_{\mathscr{V}} \omega+\Delta_{Q} v=-\mathrm{d} f . \\
\Delta_{T_{n}} f=0
\end{array} .\right.
$$

The $\left(\mathrm{H}, \omega ; \Delta_{Q}\right)$-twisted bracket $\llbracket \cdot \cdot \cdot \rrbracket^{\left(\mathrm{H}, \omega ; \Delta_{Q}\right)}$ closes on $\Gamma_{\sigma}(\mathrm{E} \mathscr{F})$, that is

$$
\mathfrak{V}, \mathfrak{W} \in \Gamma_{\sigma}(\mathrm{E} \mathscr{F}) \quad \Longrightarrow \quad \llbracket \mathfrak{V}, \mathfrak{W} \rrbracket^{\left(\mathrm{H}, \omega ; \Delta_{Q}\right)} \in \Gamma_{\sigma}(\mathrm{E} \mathscr{F}),
$$

and so it endows $\Gamma_{\sigma}(\mathrm{E} \mathscr{F})$ with the structure of an algebra over $\mathbb{R}$.

It deserves to be emphasised that the symmetry algebra $\left(\Gamma_{\sigma}(\mathrm{E} \mathscr{F}), \llbracket \cdot, \cdot \rrbracket^{\left(\mathrm{H}, \omega ; \Delta_{Q}\right)}\right)$ is not, in general, a Lie algebra. Its field-theoretic significance is reflected in 
Proposition 2.28. [Sus12, Props. 3.9 \& 5.5] Let $\mathrm{P}_{\sigma}$ be the phase space of the non-linear two-dimensional $\sigma$-model for network-field configurations $(\varphi \mid \Gamma)$ in string background $\mathfrak{B}=$ $(\mathcal{M}, \mathcal{B}, \mathcal{J})$ with target space $\mathscr{F}=M \sqcup Q \sqcup T$ on a lorentzian world-sheet $(\Sigma, \gamma)$ with defect quiver $\Gamma$. To every $\sigma$-symmetric section $\mathfrak{V} \in \Gamma_{\sigma}(\mathrm{E} \mathscr{F})$ of the total generalised tangent bundle $\mathrm{E} \mathscr{F}$ over the target space of $\mathfrak{B}$, there is associated a hamiltonian $h_{\mathfrak{V}} \in C^{\infty}\left(\mathrm{P}_{\sigma}, \mathbb{R}\right)$ generating, through Poisson brackets, the action of the Lie algebra $\mathfrak{g}$ of the symmetry group $\mathrm{G}$ on $C^{\infty}\left(\mathrm{P}_{\sigma}, \mathbb{R}\right)$, and the map $\mathfrak{V} \mapsto h_{\mathfrak{V}}$ is an $\mathbb{R}$-algebra homomorphism.

The algebra $\left(\Gamma_{\sigma}(\mathrm{E} \mathscr{F}), \llbracket \cdot, \cdot \rrbracket^{\left(\mathrm{H}, \omega ; \Delta_{Q}\right)}\right)$ of $\sigma$-model symmetries, endowed with the scalar product $(\cdot, \cdot)\lrcorner$, will be seen to play a very natural and important rôle in the characterisation of the conditions in which the rigid symmetries from $G$ can be gauged in topologically simple circumstances.

We conclude this introductory section by discussing, in the simplicial framework, some G-equivariance properties of the smooth forms

$$
\rho=\kappa_{a 2^{*}} \wedge \theta_{L 1^{*}}^{a}-\frac{1}{2} \mathrm{c}_{a b 2^{*}} \theta_{L 1^{*}}^{a} \wedge \theta_{L 1^{*}}^{b} \in \Omega^{2}(\mathrm{G} \times M),
$$

and

$$
\lambda=-k_{a 2^{*}} \theta_{L 1^{*}}^{a} \in \Omega^{1}(\mathrm{G} \times Q)
$$

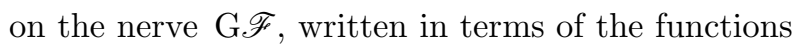

$$
\mathrm{c}_{a b}:=\imath_{a} \kappa_{b}
$$

and of the respective canonical projections $\mathrm{pr}_{1}: \mathrm{G} \times \mathscr{F} \rightarrow \mathrm{G}$ and $\mathrm{pr}_{2}: \mathrm{G} \times \mathscr{F} \rightarrow \mathscr{F}$. These objects will be of relevance in later sections, where they enter the analysis of the G-equivariance of the string background. In our discussion, we shall make extensive use of the following

Proposition 2.29. Let $\eta \in \Omega^{p}(\mathscr{M})$ be a smooth $p$-form on a $\mathrm{G}$-space $\mathscr{M}$, with the group $\mathrm{G}$ acting on $\mathscr{M}$ as in Eq. (2.10), and with vector fields $\bar{X}$ for $X \in \mathfrak{g}$ as in Definition 2.10. The identities

$$
\mathscr{M}_{h}^{*}(\imath \bar{X} \eta)=\imath \overline{\left(\operatorname{Ad}_{h^{-1}} X\right)}{ }^{\mathscr{M}} \ell_{h}^{*} \eta
$$

and

$$
\mathscr{M}_{\ell}^{*} \eta(h, m)=\left(\mathrm{e}^{-\overline{\theta_{L}(h)}} \cdot \mathscr{M}_{h}^{*} \eta\right)(m)
$$

hold for any pair $(h, m) \in \mathrm{G} \times \mathscr{M}$.

Proof. The first of the two identities is a consequence of the behaviour of the fundamental vector fields

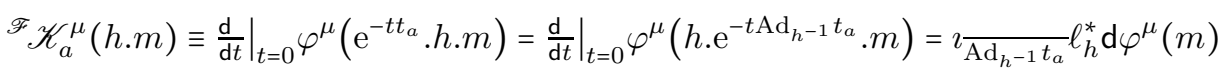

under arbitrary shifts $h \in \mathrm{G}$ of the argument $m \in \mathscr{F}$. Here, $\left\{\varphi^{\mu}\right\}$ is a local coordinate system on $\mathscr{M}$.

The second identity becomes evident upon writing it out for the basis 1 -forms on $\mathscr{M}$ associated with the coordinates $\varphi^{\mu}$ at $h . m$ and with those at $m$, to be denoted $\psi^{\nu}, \nu=$ $1,2, \ldots, \operatorname{dim} \mathscr{M}$. This also entails introducing local coordinates $X^{a}, a=1,2, \ldots, \operatorname{dim} \mathrm{G}$, at $h \in \mathrm{G}$. We then find the desired relation

$$
\begin{aligned}
\mathscr{M} \ell^{*} \mathrm{~d} \varphi^{\mu}(h, m) & =\frac{\partial \varphi^{\mu}(h \cdot m)}{\partial \psi^{\nu}(m)} \mathrm{d} \psi^{\nu}(m)+\mathrm{d} X^{a} \partial_{a} \varphi^{\mu}(h . m) \\
& \equiv \mathscr{M}_{h}^{*} \mathrm{~d} \varphi^{\mu}(m)+\left.\theta_{L}^{a}(h) \frac{\mathrm{d}}{\mathrm{d} t}\right|_{t=0} \varphi^{\mu}\left(\left(h \cdot \mathrm{e}^{t t_{a}}\right) \cdot m\right) \\
& =\mathscr{M}_{h}^{*} \mathrm{~d} \varphi^{\mu}(m)-\overline{\theta_{L}(h)} \cdot{ }^{\mathscr{M}} \ell_{h}^{*} \mathrm{~d} \varphi^{\mu}(m) \equiv \mathrm{e}^{-\overline{\theta_{L}(h)}} \cdot \ell_{h}^{*} \mathrm{~d} \varphi^{\mu}(m) .
\end{aligned}
$$

We shall also assume that the various forms on $M$ and $Q$, as well as their extensions, previously taken to be $\mathfrak{g}$-invariant resp. $\mathfrak{g}$-equivariant, are in fact G-invariant resp. G-equivariant, cf. Definition 2.12. Using the above identities for G-spaces $\mathscr{M}$ of G $\mathscr{F}$, we can readily verify 
Proposition 2.30. GSW10, Lemmas 3.11 \& 3.13] Let $\rho$ be the 2-form on $\mathrm{G} \times M$ given in $E q$. (2.30), with $\kappa=\kappa_{a} \otimes \tau^{a} \in \Omega^{1}(M) \otimes \mathfrak{g}^{*}$, defined in terms of the 1-forms $\kappa_{a}$ of Eq. (2.27), G-equivariant in the sense of Definition 2.19 and subject to the additional constraints

$$
\mathrm{c}_{b a}=-\mathrm{c}_{a b},
$$

cf. Eq. (2.32). Then, in the notation of Eq. (2.19) and Proposition 2.20, the following relations hold true:

$$
\begin{aligned}
& { }^{M} \ell_{h}^{(1) *} \rho=\rho, \quad h \in \mathrm{G} \\
& { }^{M} \delta_{\mathrm{G}}^{(1)} \rho=0, \\
& { }^{M} \delta_{\mathrm{G}}^{(0)} \mathrm{H}=\mathrm{d} \rho .
\end{aligned}
$$

The proposition was proven in Ref. GSW10. Here, on the other hand, we want to study properties of forms $\omega$ and $\lambda$.

Proposition 2.31. Under the assumptions of Proposition 2.2A, let $\omega$ be a G-invariant 2form on $Q$ satisfying Eq. (2.3) for $\mathrm{H}$ a closed $\mathrm{G}$-invariant 3-form on $M$. Finally, let $\rho$ and $\lambda$ be the corresponding forms on $\mathrm{G} \times M$ and $\mathrm{G} \times Q$, respectively, given in Eqs. (2.30) and (2.31) and related to $\omega$ as in Eq. (2.28), with $\kappa=\kappa_{a} \otimes \tau^{a} \in \Omega^{1}(M) \otimes \mathfrak{g}^{*}$ as in Proposition 2.30 and with $k=k_{a} \otimes \tau^{a} \in C^{\infty}(Q, \mathbb{R}) \otimes \mathfrak{g}^{*}$, defined in terms of functions $k_{a}$ of Eq. (2.28), G-equivariant in the sense of Definition 2.19. Then, the following relations are satisfied:

$$
\begin{aligned}
& { }^{Q} \ell_{h}^{(1) *} \lambda=\lambda, \\
& { }^{Q} \delta_{\mathrm{G}}^{(0)} \omega=-\Delta_{Q}^{(1)} \rho+\mathrm{d} \lambda, \\
& { }^{{ }} \delta_{\mathrm{G}}^{(1)} \lambda=0 .
\end{aligned}
$$

Proof. Using Eq. (2.19) and the obvious identity

$$
\left(\operatorname{Ad}_{h}^{*} \otimes \mathrm{id}_{\mathfrak{g}}\right) \theta_{L}=\left(\mathrm{id}_{\Omega^{1}(\mathrm{G})} \otimes \operatorname{Ad}_{h}\right) \theta_{L},
$$

together with the G-equivariance property of $k$,

$$
\left({ }^{Q} \ell_{h}^{(0) \star} k\right)(X)=k\left(\operatorname{Ad}_{h^{-1}} X\right),
$$

written for arbitrary $X \in \mathfrak{g}$, we find, in the natural shorthand notation $\lambda \equiv-k_{2^{*}}\left(\theta_{L 1^{*}}\right)$,

$$
{ }^{Q} \ell_{h}^{(1) *} \lambda=-\left({ }^{Q} \ell_{h}^{(0) *} k\right)_{2^{*}}\left(\left(\operatorname{Ad}_{h}^{*} \otimes \mathrm{id}_{\mathfrak{g}}\right) \theta_{L 1^{*}}\right)=-k_{2^{*}}\left(\left(\operatorname{Ad}_{h}^{*} \otimes \operatorname{Ad}_{h^{-1}}\right) \theta_{L 1^{*}}\right)=\lambda,
$$

which gives relation (2.40). Next, Eq. (2.28) and the g-equivariance property of $k$ (implied by its G-equivariance), in conjunction with the G-invariance property of $\omega$,

$$
{ }^{Q} \ell_{h}^{(0) *} \omega=\omega,
$$

and the Maurer-Cartan equation

$$
\mathrm{d} \theta_{L}^{a}=-\frac{1}{2} f_{a b c} \theta_{L}^{b} \wedge \theta_{L}^{c}
$$

yield

$$
\begin{aligned}
{ }^{Q} d_{1}^{(1) *} \omega(g, m) & \equiv{ }^{Q} \ell^{(0) *} \omega(g, m)=\left(\mathrm{e}^{-\overline{\theta_{L}(g)}} \cdot{ }^{Q} \ell_{g}^{(0) *} \omega\right)(m)=\mathrm{e}^{-\overline{\theta_{L}(g)}} \omega(m) \\
& =\omega(m)+\theta_{L}^{a}(g) \wedge\left(\mathrm{d} k_{a}+\Delta_{Q}^{(0)} \kappa_{a}\right)(m)+\frac{1}{2} \theta_{L}^{a} \wedge \theta_{L}^{b}(g) \imath_{a}\left(\mathrm{~d} k_{b}+\Delta_{Q}^{(0)} \kappa_{b}\right)(m) \\
& =\left({ }^{Q} d_{0}^{(1)} \omega-\Delta_{Q}^{(1)} \rho+\mathrm{d} \lambda\right)(g, m),
\end{aligned}
$$

which is Eq. 2.41). Finally, taking, say, Eq. 2.41), we note that it implies, by virtue of Eqs. (2.23) and (2.38), the identity

$$
\mathrm{d}^{Q} \delta_{\mathrm{G}}^{(1)} \lambda \equiv{ }^{Q} \delta_{\mathrm{G}}^{(1)} \circ{ }^{Q} \delta_{\mathrm{G}}^{(0)} \omega+{ }^{Q} \delta_{\mathrm{G}}^{(1)} \circ \Delta_{Q}^{(1)} \rho=\Delta_{Q}^{(2)} \circ{ }^{M} \delta_{\mathrm{G}}^{(1)} \rho=0 .
$$

Using

$$
\theta_{L}(g \cdot h)=\theta_{L}(h)+\operatorname{Ad}_{h^{-1}} \theta_{L}(g),
$$


we demonstrate that the de Rham cocycle ${ }^{Q} \delta_{\mathrm{G}}^{(1)} \lambda$ actually trivialises as

$$
\begin{aligned}
{ }^{Q} d_{1}^{(2) *} \lambda\left(g_{2}, g_{1}, m\right) & \equiv \lambda\left(g_{2} \cdot g_{1}, m\right)=-k\left(\theta_{L}\left(g_{1}\right)+\operatorname{Ad}_{g_{1}^{-1}} \theta_{L}\left(g_{2}\right)\right)(m) \\
& =-k\left(\theta_{L}\left(g_{1}\right)\right)(m)-{ }^{Q} \ell_{g_{1}}^{(0) *} k\left(\theta_{L}\left(g_{2}\right)\right)(m) \\
& =\left({ }^{Q} d_{0}^{(2) *} \lambda+{ }^{Q} d_{2}^{(2) *} \lambda\right)\left(g_{2}, g_{1}, m\right),
\end{aligned}
$$

which, upon dropping the point dependence, yields relation (2.42). This completes the proof of the proposition.

\section{The Coupling to gauge fields: topologically trivial SeCtor}

Rigid symmetries of a field theory can sometimes be gauged, i.e. promoted to the rank of local ones. In the $\sigma$-model context, in the presence of extra differential-geometric structures on the target space, it has to be ensured that the action of the symmetry group on the target space lifts to those structures. Thus, e.g., in the case in which the smooth target space $\mathscr{F}$ carries a metric and a gerbe, the action should be isometric and such that the gerbe is equivariant with respect to it in an appropriate sense. The gauging of group G of rigid symmetries involves a coupling to background gauge fields, given by connections on a principal G-bundle $\mathrm{P}$ over the space-time (in our case, the world-sheet) $\Sigma$. The topologically trivial sector corresponds to the case with a trivial G-bundle $\mathrm{P}=\Sigma \times G$. In this case, the gauge fields are represented by global 1-forms on the world-sheet with values in the Lie algebra $\mathfrak{g}$ of the symmetry group G. Somewhat misleadingly, we shall call such gauge fields topologically trivial in what follows. It may turn out to be physically appropriate, however, not to restrict attention to the case of trivial (or trivializable) G-bundles and to consider topologically nontrivial sectors with nontrivial G-bundles $\mathrm{P}$ over $\Sigma$. For such bundles, the inclusion of space-time gauge fields is accompanied by the replacement of the original fields of the theory by (global) sections of the associated bundle $\mathrm{P} \times_{\mathrm{G}} \mathscr{F}$. In both topologically trivial and topologically nontrivial sectors, the gauging has to take into account topological issues associated with large gauge transformations non-homotopic to the identity transformation. The present section is dedicated to a detailed study of the gauging procedure in the more straightforward case of the topologically trivial sector. Already this analysis will lead to some interesting compatibility conditions for a consistent lift of the geometric symmetries on $\mathscr{F}$ to the geometric and cohomological structures over the target space. We shall provide a precise algebraic, cohomological and canonical characterisation of the ensuing constraints for the consistent gauging.

3.1. Insights from the study of trivial backgrounds. In order to develop some intuition as to the possible nature of the coupling between the world-sheet gauge fields and the targetspace structures, we further simplify the physical setting by taking all target-space structures to be trivial, that is described by tensor fields, the latter being further assumed G-invariant. Thus, we set

$$
\begin{array}{llll}
\mathcal{G}=I_{\mathrm{B}}, & \mathrm{B} \in \Omega^{2}(M), & \text { with } & { }^{M} \ell_{g}^{*} \mathrm{~B}=\mathrm{B}, \\
\Phi=J_{\mathrm{P}}, & \mathrm{P} \in \Omega^{1}(Q), & \text { with } & { }{ }^{*} \ell_{g}^{*} \mathrm{P}=\mathrm{P}, \\
\phi_{n}=K_{\mathrm{f}_{n}}, & \mathrm{f}_{n} \in C^{\infty}\left(T_{n}, \mathrm{U}(1)\right), & \text { with } & { }^{T_{n}} \ell_{g}^{*} \mathrm{f}_{n}=\mathrm{f}_{n},
\end{array}
$$

for arbitrary $g \in \mathrm{G}$, with, as previously, $I_{\mathrm{B}}$ the trivial gerbe with (global) curving $\mathrm{B}, J_{\mathrm{P}}$ the trivial bi-brane defined by the smooth 1 -form $\mathrm{P}$, and $K_{\mathrm{f}_{n}}$ the trivial inter-bi-branes determined by the respective smooth $\mathrm{U}(1)$-valued functions $\mathrm{f}_{n}$ on $T_{n}$. The tensors entering the above definitions are subject to the relations

$$
\Delta_{Q} \mathrm{~B}=\mathrm{dP}-\omega, \quad \Delta_{T_{n}} \mathrm{P}-\mathrm{id} \log \mathrm{f}_{n}=0 .
$$

The Feynman amplitude (2.8) now takes the simple form

$$
\mathscr{A}[(\varphi \mid \Gamma) ; \gamma]=\exp \left[-\frac{1}{2} \int_{\Sigma} \mathrm{g}\left(\mathrm{d} \varphi \hat{\wedge} \star_{\gamma} \mathrm{d} \varphi\right)+\mathrm{i} \int_{\Sigma} \varphi^{*} \mathrm{~B}+\mathrm{i} \int_{\Gamma}\left(\left.\varphi\right|_{\Gamma}\right)^{*} \mathrm{P}\right] \prod_{\jmath \in \mathfrak{V}_{\Gamma}} \varphi^{*} \mathrm{f}_{n_{\jmath}}(\jmath),
$$


where the product is taken over the set $\mathfrak{V}_{\Gamma}$ of defect junctions. Note that we may take

$$
\kappa_{a}=\imath_{a} \mathrm{~B}, \quad k_{a}=\imath_{a} \mathrm{P}
$$

in this case, and this choice is consistent with Proposition 2.24 as

$$
\begin{aligned}
\Delta_{Q} \kappa_{a} & \equiv \Delta_{Q}\left(\imath_{a} \mathrm{~B}\right)=\imath_{a} \Delta_{Q} \mathrm{~B}=\imath_{a}(\mathrm{dP}-\omega)=-\mathrm{d}\left(\imath_{a} \mathrm{P}\right)-\imath_{a} \omega \\
& \equiv-\mathrm{d} k_{a}-\imath_{a} \omega \\
\Delta_{T_{n}} k_{a} & \equiv \Delta_{T_{n}}\left(\imath_{a} \mathrm{P}\right)=\imath_{a} \Delta_{T_{n}} \mathrm{P}=\mathrm{i} \imath_{a} \mathrm{~d} \log \mathrm{f}_{n}=0
\end{aligned}
$$

Finally, we readily check the identities

$$
\begin{aligned}
& \mathscr{L}_{a} \kappa_{b}=f_{a b c} \kappa_{c}, \\
& \mathscr{L}_{a} k_{b}=f_{a b c} k_{c}
\end{aligned}
$$

and

$$
\mathrm{c}_{b a}=-\mathrm{c}_{a b} .
$$

Actually, in consequence of the assumed G-invariance of the background, a little more is true: With the help of Proposition 2.29, we readily establish the G-equivariance of $\kappa=\kappa_{a} \otimes \tau^{a}$ and $k=k_{a} \otimes \tau^{a}$.

Let $\mathrm{A}=A_{u}^{a} \mathrm{~d} \sigma^{u} \otimes t_{a} \in \Omega^{1}(\Sigma) \otimes \mathfrak{g}$ be a (topologically trivial) world-sheet gauge field, undergoing a transformation

$$
\mathrm{A} \mapsto \mathrm{Ad}_{\chi} \mathrm{A}-\mathrm{d} \chi \chi^{-1} \equiv{ }^{\chi} \mathrm{A}
$$

under a gauge transformation $\chi \in C^{\infty}(\Sigma, \mathrm{G})$ that maps a $\sigma$-model field according to

$$
\varphi \mapsto{ }^{\mathscr{F}} \ell(\chi, \varphi) \equiv \chi \cdot \varphi \text {. }
$$

The standard 'minimal-coupling' recipe, familiar from models of point-particle physics, is tantamount to the replacement of the vector field-valued world-sheet forms $\mathrm{d} \varphi$ in the term involving the world-sheet metric by

$$
\mathrm{d} \varphi(\sigma) \mapsto \mathrm{e}^{-\overline{\mathrm{A}(\sigma)}} \cdot \mathrm{d} \varphi(\sigma)=\left(\partial_{u} \varphi^{\mu}-A_{u}^{a} \mathscr{K}_{a}^{\mu}(\varphi)\right)(\sigma) \mathrm{d} \sigma^{u} \otimes \partial_{\mu}=: D_{\mathrm{A}} \varphi(\sigma),
$$

and of the target-space forms $\mathrm{B}$ and $\mathrm{P}$ in the 'topological' term by the corresponding objects

$$
\mathrm{B} \mapsto \mathrm{e}^{-\overline{\mathrm{A}}_{1^{*}}} \cdot \mathrm{B}_{2^{*}}, \quad \mathrm{P} \mapsto \mathrm{e}^{-\overline{\mathrm{A}}_{1^{*}}} \cdot \mathrm{P}_{2^{*}}
$$

on the product space $\Sigma \times \mathscr{F}$ (the latter coming with the canonical projections $\operatorname{pr}_{1}: \Sigma \times \mathscr{F} \rightarrow \Sigma$ and $\operatorname{pr}_{2}: \Sigma \times \mathscr{F} \rightarrow \mathscr{F}$, indicated by the subscripts), to be subsequently pulled back to $\Sigma$ along the map

$$
\phi=\left(\operatorname{id}_{\Sigma}, \varphi\right): \Sigma \rightarrow \Sigma \times \mathscr{F} .
$$

We have

Proposition 3.1. In the notation of Definition 2.9, and for a $\mathrm{G}$-invariant topologically trivial string background $\mathfrak{B}$ defined by Eqs. (3.1)-(3.3), let $\mathrm{A} \in \Omega^{1}(\Sigma) \otimes \mathfrak{g}$ be a topologically trivial gauge field on $\Sigma$. The gauged non-linear $\sigma$-model amplitudes for network-field configurations $(\varphi \mid \Gamma)$ in background $\mathfrak{B}$, given by the expression

$$
\begin{gathered}
\widehat{\mathscr{A}}[(\varphi \mid \Gamma) ; \mathrm{A}, \gamma] \\
=\exp \left[-\frac{1}{2} \int_{\Sigma} \mathrm{g}\left(D_{\mathrm{A}} \varphi^{\wedge},{ }^{\star}{ }_{\gamma} D_{\mathrm{A}} \varphi\right)+\mathrm{i} \int_{\Sigma} \phi^{*} \mathrm{e}^{-\overline{\mathrm{A}}_{1 *}} \cdot \mathrm{B}_{2^{*}}+\mathrm{i} \int_{\Gamma}\left(\left.\phi\right|_{\Gamma}\right)^{*} \mathrm{e}^{-\overline{\mathrm{A}}_{1 *}} \cdot \mathrm{P}_{2^{*}}\right] \prod_{\jmath \in \mathfrak{V}_{\Gamma}} \varphi^{*} \mathrm{f}_{n_{\jmath}}(\jmath)
\end{gathered}
$$

written in terms of the extended world-sheet field $\phi$ of Eq.(3.11), are invariant under simultaneous gauge transformations $(\varphi, \mathrm{A}) \mapsto\left(\chi \cdot \varphi,{ }^{\chi} \mathrm{A}\right)$ for $\chi \in C^{\infty}(\Sigma, \mathrm{G})$. 
Proof. The 'metric' and 'topological' terms are independently invariant under the gauge transformations. We begin by demonstrating the invariance of the former. To this end, we examine the transformed covariant derivative,

$$
D_{\mathrm{A}} \varphi^{\mu} \mapsto D_{\chi \mathrm{A}}(\chi \cdot \varphi)^{\mu},
$$

where both $\varphi$ and $\chi$ in the last expression are functions of the world-sheet coordinates $\sigma^{u}, u=1,2$. Taking into account this $\sigma^{u}$-dependence of $\varphi$ and that of $\chi$, respectively, we decompose the transformed world-sheet differential $\mathrm{d}(\chi \cdot \varphi)^{\mu}$ into two terms as in Eq. (2.36), whereby we obtain, using Eq. 2.35),

$$
\begin{aligned}
D_{\chi_{\mathrm{A}}}(\chi \cdot \varphi)^{\mu} & =\left(\ell_{\chi}^{*} \mathrm{~d} \varphi^{\mu}-\imath \overline{\chi^{*} \theta_{L}} \ell_{\chi}^{*} \mathrm{~d} \varphi^{\mu}\right)-\left(\mathrm{Ad}_{\chi} \mathrm{A}-\mathrm{d} \chi \chi^{-1}\right)^{a} \imath \overline{\mathrm{Ad}_{\chi^{-1} t_{a}}} \ell_{\chi}^{*} \mathrm{~d} \varphi^{\mu} \\
& =\left(\ell_{\chi}^{*} \mathrm{~d} \varphi^{\mu}-\imath \overline{\chi^{*} \theta_{L}} \ell_{\chi}^{*} \mathrm{~d} \varphi^{\mu}\right)-\imath \overline{\mathrm{A}-\chi^{-1} \mathrm{~d} \chi} \ell_{\chi}^{*} \mathrm{~d} \varphi^{\mu} \\
& =\left(\ell_{\chi}^{*} \mathrm{~d} \varphi^{\mu}-\imath \overline{\mathrm{A}} \ell_{\chi}^{*} \mathrm{~d} \varphi^{\mu}\right)=\frac{\partial(\chi \cdot \varphi)^{\mu}}{\partial \varphi^{\nu}} D_{\mathrm{A}} \varphi^{\nu} .
\end{aligned}
$$

This justifies the name given to the object $D_{\mathrm{A}} \varphi^{\mu}$ and straightforwardly implies the gauge invariance of the 'metric' term by virtue of the G-invariance of the target-space metric.

Next, we pass to the 'topological' term. Its gauge invariance is a simple corollary to the following observation.

Lemma 3.2. Let $\eta$ be a G-invariant $p$-form on $\mathscr{F}$. Then, in the above notation,

$$
(\chi \cdot \phi)^{*} \mathrm{e}^{-\overline{\mathrm{X}_{1^{*}}}} \cdot \eta_{2^{*}}=\phi^{*} \mathrm{e}^{-\overline{\mathrm{A}_{1^{*}}}} \cdot \eta_{2^{*}} .
$$

Proof. Our proof employs a technique that will turn out useful later on. It associates to a gauge transformation

$$
\chi: \Sigma \rightarrow \mathrm{G}
$$

a transformation on the product space $\Sigma \times \mathscr{F}$

$$
\begin{aligned}
L_{\chi}: \Sigma \times \mathscr{F} & \rightarrow \sum \times \mathscr{F} \\
(\sigma, m) & \mapsto(\sigma, \chi(\sigma) . m),
\end{aligned}
$$

and subsequently decomposes the lift as

$$
\begin{aligned}
L_{\chi}: \Sigma \times \mathscr{F} & \stackrel{K_{\chi}}{\longrightarrow} \sum \times \mathrm{G} \times \mathscr{F} \stackrel{\mathrm{id}_{\Sigma} \times \mathscr{F}_{\ell}}{\longmapsto} \Sigma \times \mathscr{F} \\
(\sigma, m) & \longmapsto(\sigma, \chi(\sigma), m) \stackrel{\longmapsto}{\longmapsto}(\sigma, \chi(\sigma) . m) .
\end{aligned}
$$

Upon invoking Proposition 2.29, we find the sought-after result

$$
\begin{aligned}
\left((\chi \cdot \phi)^{*} \mathrm{e}^{-\overline{\chi_{\mathrm{A}_{1 *}}}} \cdot \eta_{2^{*}}\right)(\sigma) & =\left(\left(L_{\chi} \circ \phi\right)^{*} \mathrm{e}^{-\overline{\mathrm{X}_{1^{*}}}} \cdot \eta_{2^{*}}\right)(\sigma)=\phi^{*}\left(\mathrm{e}^{-\overline{\left(\mathrm{Ad}_{\chi^{-1}} \chi \mathrm{A}\right)} 1^{*}} \cdot L_{\chi}^{*} \eta_{2^{*}}\right)(\sigma) \\
& =\mathrm{e}^{\left.-\overline{\left(\mathrm{Ad}_{\chi^{-1}} \chi \mathrm{A}\right.}\right)(\sigma)} \cdot \mathrm{e}^{-\overline{\chi^{*} \theta_{L}(\sigma)}} \cdot{ }^{\mathscr{F}} \ell_{\chi(\sigma)}^{*} \varphi^{*} \eta(\sigma)=\mathrm{e}^{-\overline{\mathrm{A}(\sigma)}} \cdot \varphi^{*} \eta(\sigma) \\
& \equiv \phi^{*}\left(\mathrm{e}^{-\overline{\mathrm{A}_{1^{*}}}} \cdot \eta_{2^{*}}\right)(\sigma) .
\end{aligned}
$$

It is now clear that the explicit form of the 'minimal coupling' used, in conjunction with the assumed G-invariance of the string background imply the thesis of the proposition.

With view to a subsequent generalisation, let us rewrite the Feynman amplitude (3.12) in the more suggestive form

$$
\begin{gathered}
\widehat{\mathscr{A}}[(\varphi \mid \Gamma) ; \mathrm{A}, \gamma] \\
=\exp \left[-\frac{1}{2} \int_{\Sigma} \mathrm{g}_{\mathrm{A}}\left(\mathrm{d} \phi \hat{\wedge} \star_{\gamma} \mathrm{d} \phi\right)+\mathrm{i} \int_{\Sigma} \phi^{*}\left(\mathrm{~B}_{2^{*}}+\rho_{\mathrm{A}}\right)+\mathrm{i} \int_{\Gamma}\left(\left.\phi\right|_{\Gamma}\right)^{*}\left(\mathrm{P}_{2^{*}}+\lambda_{\mathrm{A}}\right)\right] \prod_{\jmath \in \mathfrak{V}_{\Gamma}} \varphi^{*} \mathrm{f}_{n_{\jmath}}(\jmath)
\end{gathered}
$$

upon introducing the forms

$$
\rho_{\mathrm{A}}:=\kappa_{a 2^{*}} \wedge \mathrm{A}_{1^{*}}^{a}-\frac{1}{2} \mathrm{c}_{a b 2^{*}} \mathrm{~A}_{1^{*}}^{a} \wedge \mathrm{A}_{1^{*}}^{b} \in \Omega^{2}(\Sigma \times M),
$$




$$
\lambda_{\mathrm{A}}:=-k_{a 2^{*}} \mathrm{~A}_{1^{*}}^{a} \in \Omega^{1}(\Sigma \times Q),
$$

and the (possibly degenerate) metric tensor

$$
\mathrm{g}_{\mathrm{A}}:=\mathrm{g}_{2^{*}}-\mathrm{K}_{a 2^{*}} \otimes \mathrm{A}_{1^{*}}^{a}-\mathrm{A}_{1^{*}}^{a} \otimes \mathrm{K}_{a 2^{*}}+\mathrm{h}_{a b 2^{*}}\left(\mathrm{~A}^{a} \otimes \mathrm{A}^{b}\right)_{1^{*}}
$$

on the 'extended' target-space $\Sigma \times \mathscr{F}$. The latter tensor is defined in terms of the 1-forms

$$
\mathrm{K}_{a}:=\mathrm{g}\left({ }^{M} \mathscr{K}_{a}, \cdot\right) \in \Omega^{1}(M)
$$

and of the symmetric tensor

$$
\mathrm{h}_{a b}:=\mathrm{g}\left({ }^{M} \mathscr{K}_{a},{ }^{M} \mathscr{K}_{b}\right),
$$

and implicitly understood to act on the second tensor factor in

$$
\mathrm{d} \phi(\sigma)=\left(\mathrm{d} \sigma^{u} \otimes \partial_{u},\left.\partial_{u} \varphi^{\mu}(\sigma) \mathrm{d} \sigma^{u} \otimes \partial_{\mu}\right|_{\varphi(\sigma)}\right) .
$$

The lesson to be drawn from the above analysis can now be phrased as follows: The coupling of a topologically trivial gauge field $\mathrm{A}$ to the $\sigma$-model fields boils down to replacing the original quadruple $(\mathrm{g}, \mathcal{G}, \Phi, \varphi)$ over the target space $\mathscr{F}$ in the amplitude by the corrected one $\left(\mathrm{g}_{\mathrm{A}}, \mathcal{G}_{\mathrm{A}}, \Phi_{\mathrm{A}}, \phi\right)$ over $\Sigma \times \mathscr{F}$, with $\mathrm{g}_{\mathrm{A}}$ given by Eq. (3.17),

$$
\mathcal{G}_{\mathrm{A}}:=\mathcal{G}_{2^{*}} \otimes I_{\rho_{\mathrm{A}}}, \quad \Phi_{\mathrm{A}}:=\Phi_{2 *} \otimes J_{\lambda_{\mathrm{A}}},
$$

and with $\phi$ defined by Eq. (3.11).

3.2. An Ansatz for nontrivial backgrounds, and constraints for a consistent gauging. Passing to general backgrounds, while keeping the world-sheet gauge field topologically trivial, we imitate the simple prescription established at the end of the previous section and study the ensuing constraints. This is the route taken in Ref. [GSW10] in the defect-free case. It was first proposed by Jack et al. in Ref. [JJMO90] and by Hull and Spence in Ref. HS89] for world-sheets without defects, and by Figueroa-O'Farrill and Mohammedi in Ref. [FOM05] for those with boundary defects, where the circumstances were examined in which the symmetry generated by the vector fields ${ }^{M} \mathscr{K}_{a}$ and ${ }^{Q} \mathscr{K}_{a}$ can be gauged. The constraints for the possible coupling terms derived below agree, in the domain of common applicability, with those deduced in the earlier papers, however, the present method of derivation is slightly more straightforward.

Let us recall, by way of a warm-up, the result of Ref. [GSW10], obtained by postulating a simple form of the coupling of the standard Wess-Zumino amplitude in the absence of defects,

$$
\mathscr{A}_{\mathrm{WZ}}(\varphi)=\operatorname{Hol}_{\mathcal{G}}(\varphi)
$$

to topologically trivial gauge fields, and by subsequently using the presumed infinitesimal gauge-invariance of the extended amplitude to fix its free parameters. More specifically, we have

Proposition 3.3. [JJMO90, HS89] [GSW10, Prop. 3.1] Consider $a \mathfrak{g}^{*}$-valued 1-form and a $\mathfrak{g}^{*} \wedge \mathfrak{g}^{*}$-valued function

$$
\alpha=\alpha_{a} \otimes \tau^{a} \in \Omega^{1}(M) \otimes \mathfrak{g}^{*}, \quad \quad \beta=\beta_{a b} \otimes\left(\tau^{a} \wedge \tau^{b}\right) \in \Omega^{0}(M) \otimes\left(\mathfrak{g}^{*} \wedge \mathfrak{g}^{*}\right)
$$

on $M$, written in the notation of Definition 2.19. An extension

$$
\widehat{\mathscr{A}}_{\mathrm{WZ}}(\varphi ; \mathrm{A})=\mathrm{e}^{\mathrm{i} \int_{\Sigma} \phi^{*} \varsigma_{\mathrm{A}}} \mathscr{A}_{\mathrm{WZ}}(\varphi)
$$

of $\mathscr{A}_{\mathrm{WZ}}(\varphi)$, expressed in terms of the map $\phi=\left(\operatorname{id}_{\Sigma}, \varphi\right): \Sigma \rightarrow \Sigma \times M$ and of a 2-form

$$
\varsigma_{\mathrm{A}}=\alpha_{a 2^{*}} \wedge \mathrm{A}_{1^{*}}^{a}-\frac{1}{2} \beta_{a b 2^{*}} \mathrm{~A}_{1^{*}}^{a} \wedge \mathrm{A}_{1^{*}}^{b}
$$

on $\Sigma \times M$, is invariant under infinitesimal gauge transformations

$$
\varphi \mapsto \varphi+\imath \bar{\Lambda} \mathrm{d} \varphi, \quad \mathrm{A} \mapsto \mathrm{A}+\mathrm{d} \Lambda-[\Lambda, \mathrm{A}],
$$

with $\Lambda=\Lambda^{a} t_{a}$ and $\bar{\Lambda}=\Lambda^{a} \mathscr{K}_{a}$ for $\Lambda^{a} \in C^{\infty}(\Sigma, \mathbb{R})$, iff the following conditions:

$$
\begin{array}{cr}
\imath_{\bar{X}} \mathrm{H}=-\mathrm{d} \alpha(X), & \beta(X \wedge Y)=\imath \bar{X}_{\bar{X}} \alpha(Y), \\
\mathscr{L}_{\bar{X}} \alpha(Y)=\alpha([X, Y]), & \imath_{\bar{X}} \alpha(Y)=-\imath \bar{Y} \alpha(X)
\end{array}
$$

are satisfied for all $X, Y \in \mathfrak{g}$. 
It yields the all-important

Corollary 3.4. In the notation of Proposition 3.9, the formula

$$
\widehat{\mathscr{A}}_{\mathrm{WZ}}(\varphi ; \mathrm{A})=\mathrm{e}^{\mathrm{i} \int_{\Sigma} \phi^{*} \rho_{\mathrm{A}}} \mathscr{A}_{\mathrm{WZ}}(\varphi)
$$

with $\rho_{\mathrm{A}}$ given by Eq.(3.15) defines an infinitesimally gauge-invariant extension of $\mathscr{A}_{\mathrm{WZ}}(\varphi)$ iff the 1-forms $\kappa_{a}$ satisfy conditions (2.27), (3.6) and (3.8).

Constraints (2.27), (3.6) and (3.8), in conjunction with the closedness of $\mathrm{H}$, admit a number of interpretations. Below, we point out two of them, a cohomological one and a symplectic one. Two more, of a gerbe-theoretic and generalised-geometric flavour, respectively, are postponed until Sections 6 and 8 , where we come to discuss the infinitesimal equivariance of the string background and the groupoid structure underlying rigid $\sigma$-model symmetries.

Remark 3.5. Obstructions to gauging infinitesimal (rigid) symmetries of the defect-free $\sigma$ model (or, equivalently, of those of its symmetries that belong to the connected component of the group unit in G) were first discussed in the framework of $\mathfrak{g}$-equivariant cohomology by Figueroa-O'Farrill and Stanciu in Refs. FOS94a, FOS94b, cf. also Refs. Wit92, Wu93. The upshot of their analysis is the following

Proposition 3.6. Let $\mathrm{H}$ be a smooth closed G-invariant 3-form on a G-space M. Relations (2.27), (3.6) and (3.8) are satisfied iff $\mathrm{H}$ admits a $\mathfrak{g}$-equivariantly closed extension

$$
\widehat{\mathrm{H}}=\mathrm{H}-\kappa, \quad \kappa \in \Omega^{1}(M) \otimes \mathfrak{g}^{*}
$$

given in terms of the $\mathfrak{g}$-equivariant 1-form $\kappa=\kappa_{a} \otimes \tau^{a}$ in the Cartan model of the $\mathfrak{g}$-equivariant cohomology of $M$.

Above, the Cartan model is taken with the $\mathfrak{g}$-equivariant differential defined on $\mathfrak{g}$-equivariant $p$-forms $\eta$, polynomially dependent on $X \in \mathfrak{g}$, by the formula

$$
\widehat{\mathrm{d}} \eta(X)=\mathrm{d} \eta(X)-\imath \bar{X} \eta(X) .
$$

An equivalent description is provided by the Weil model of the $\mathfrak{g}$-equivariant cohomology.

Proposition 3.7. GSW10, Lemma 3.9] Let $M$ be a G-space with vector fields ${ }^{M} \mathscr{K}_{a}$ as in Definition 2.10. Furthermore, let $\mathrm{A}$ be a $\mathfrak{g}$-valued 1-form on an oriented two-dimensional manifold $\Sigma$, and denote $\mathrm{F}=\mathrm{dA}+\mathrm{A} \wedge \mathrm{A} \in \Omega^{2}(\Sigma) \otimes \mathfrak{g}$. The Weil transform $\mathrm{H}_{\mathrm{A}}=\mathrm{e}^{-\overline{\mathrm{A}_{1^{*}}}} \cdot \widehat{\mathrm{H}}_{2^{*}}\left(\mathrm{~F}_{1^{*}}\right)$ of the $\mathfrak{g}$-equivariantly closed extension $\widehat{\mathrm{H}}$ of a $\mathrm{G}$-invariant 3-form $\mathrm{H}$ on $M$, given in Proposition 3.0, reads

$$
\mathrm{H}_{\mathrm{A}}=\mathrm{H}_{2^{*}}+\mathrm{d} \rho_{\mathrm{A}},
$$

where $\rho_{\mathrm{A}}$ is the 2-form on $\Sigma \times M$ defined in Eq. (3.15). Hence, $\mathrm{H}_{\mathrm{A}}$ is the curvature 3-form of the gerbe $\mathcal{G}_{\mathrm{A}}=\mathcal{G}_{2^{*}} \otimes I_{\rho_{\mathrm{A}}}$.

Remark 3.8. The discussion of a symplectic realisation of the rigid symmetries of the $\sigma$-model on its phase space was originally put in the context of generalised geometry of Refs. Hit03, Gua03] by Alekseev and Strobl in Ref. [AS05]. The more complete picture, incorporating, in particular, bi-brane data, is presented in Ref. [Sus12]. Here, we merely cite

Proposition 3.9. AS05 Sus12, Prop. 3.10] The symplectic realisation, mentioned in Proposition 2.28, of the symmetry algebra $\mathfrak{g}$ on the phase space $\mathrm{P}_{\sigma}$ of the non-linear two-dimensional $\sigma$-model on a lorentzian world-sheet with an empty defect quiver is hamiltonian iff condition (3.6) is satisfied. If, in addition, also Eq. (3.8) holds true, the equitemporal Poisson bracket of the associated Noether currents is anomaly-free and the resulting Poisson algebra of Noether currents is isomorphic with $\mathfrak{g}$.

Prior to taking up the case of a non-empty defect quiver, we point out that relations (2.27), (3.6) and (3.8), in conjunction with closedness of $\mathrm{H}$, imply further identities

$$
\begin{aligned}
\imath_{a} \imath_{b} \mathrm{H} & =-f_{a b c} \kappa_{c}+\mathrm{dc}_{a b}, \\
\imath_{a} \imath_{b} \imath_{c} \mathrm{H} & =-f_{b c d} \mathrm{c}_{a d}-f_{a b d} \mathrm{c}_{c d}+f_{a c d} \mathrm{c}_{b d},
\end{aligned}
$$


to be invoked later in our discussion.

We may now reiterate the previous reasoning in the presence of a defect quiver $\Gamma$, and for a general background $\mathfrak{B}=(\mathcal{M}, \mathcal{B}, \mathcal{J})$, whereby the starting point is, this time, the $\Gamma$-corrected Wess-Zumino amplitude

$$
\mathscr{A}_{\mathrm{WZ}}(\varphi \mid \Gamma)=\operatorname{Hol}_{\mathcal{G}, \Phi,\left(\varphi_{n}\right)}(\varphi \mid \Gamma) .
$$

Taking into account Proposition 3.3, we obtain

Proposition 3.10. Let $\mathscr{A}_{\mathrm{WZ}}(\varphi \mid \Gamma)$ be the Wess-Zumino amplitude (3.24) defining the 'topological' term of the $\sigma$-model (2.8) with the target as in Proposition 3.5, and with a bi-brane $\mathcal{B}=\left(Q, \iota_{\alpha}, \omega, \Phi\right)$ of a world-volume given by a $\mathrm{G}$-space $Q$ and a $\mathrm{G}$-invariant curvature $\omega$. Consider a $\mathfrak{g}$-valued function $\gamma=\gamma_{a} \otimes \tau^{a} \in C^{\infty}(Q, \mathbb{R}) \otimes \mathfrak{g}^{*}$ on $Q$. In the notation of Proposition 3.3, an extension

$$
\widehat{\mathscr{A}} \mathrm{WZ}[(\varphi \mid \Gamma) ; \mathrm{A}]=\mathrm{e}^{\mathrm{i} \int_{\Sigma} \phi^{*} \rho_{\mathrm{A}}} \mathrm{e}^{\mathrm{i} \int_{\Gamma}\left(\left.\phi\right|_{\Gamma}\right)^{*} \mu_{\mathrm{A}}} \mathscr{A}_{\mathrm{WZ}}(\varphi \mid \Gamma)
$$

of $\mathscr{A}_{\mathrm{WZ}}(\varphi \mid \Gamma)$, expressed in terms of the map $\phi=\left(\operatorname{id}_{\Sigma}, \varphi\right)$, of the 2-form $\rho_{\mathrm{A}}$ given by Eq. (3.15), and of a 1-form

$$
\mu_{\mathrm{A}}=-\gamma_{a 2^{*}} \mathrm{~A}_{1^{*}}^{a}
$$

on $\Sigma \times Q$, is invariant under infinitesimal gauge transformations (3.20), written for $\bar{\Lambda}=$ $\Lambda^{a \mathscr{F}} \mathscr{K}_{a}$ with the fundamental vector fields $\mathscr{F}^{K_{a}}$, iff, in addition to Eqs. (2.27), (3.6) and (3.8), the following conditions:

$$
\begin{gathered}
\imath_{\bar{X}} \omega+\left(\Delta_{Q} \kappa+\mathrm{d} \gamma\right)(X)=0, \quad \Delta_{T_{n}} \gamma(X)=0, \\
\mathscr{L}_{\bar{X}} \gamma(Y)=\gamma([X, Y])
\end{gathered}
$$

are satisfied for all $X, Y \in \mathfrak{g}$.

Proof. Writing out the variation of the extension under transformation (3.20), we find, in addition to the bulk term whose vanishing is ensured by the argument of Proposition 3.3, a collection of terms localised on $\Gamma$,

$$
\begin{array}{r}
\int_{\Gamma}\left(\left.\phi\right|_{\Gamma}\right)^{*}\left[\Lambda_{1^{*}}^{a}\left(\imath_{a} \omega\right)_{2^{*}}-\Lambda_{1^{*}}^{a}\left(\underline{\imath}_{a} \underline{\Delta}_{Q} \rho_{\mathrm{A}}\right)-\Lambda_{1^{*}}^{a}\left(\imath_{a} \mathrm{~d} \gamma_{b}\right)_{2^{*}} \wedge \mathrm{A}_{1^{*}}^{b}\right] \\
+\int_{\Gamma}\left(\left.\phi\right|_{\Gamma}\right)^{*}\left[\Lambda_{1^{*}}^{a} \underline{\Delta}_{Q}\left(\kappa_{a 2^{*}}+\mathrm{c}_{a b 2^{*}} \mathrm{~A}_{1^{*}}^{b}\right)+\Lambda_{1^{*}}^{a} \mathrm{~d} \gamma_{a 2^{*}}+\gamma_{2^{*}}\left([\Lambda, \mathrm{A}]_{1^{*}}\right)\right] \\
-\sum_{\jmath \in \mathfrak{V}_{\Gamma}} \Lambda_{1^{*}}^{a}\left(\Delta_{T_{n_{\jmath}}} \gamma_{a}\right)_{2^{*}}(\jmath),
\end{array}
$$

with $\underline{\Delta}_{Q}=\left(\operatorname{id}_{\Gamma} \times \iota_{2}\right)^{*}-\left(\operatorname{id}_{\Gamma} \times \iota_{1}\right)^{*}$ and $\underline{\mathscr{K}}_{a}(\sigma, q)={ }^{Q} \mathscr{K}_{a}(q)$, and with $\underline{\imath}_{a}=\imath_{Q_{\mathscr{K}_{a}}}$. In the above expression, the first term is the defect contribution to Eq. (2.25), the second one comes from the exact term in the variation of the bulk extension $\int_{\Sigma} \phi^{*} \rho_{\mathrm{A}}$, the third one is the variation of the target-space tensors in the defect extension $\int_{\Gamma}\left(\left.\phi\right|_{\Gamma}\right)^{*} \mu_{\mathrm{A}}$, the fourth one is produced - upon integration by parts - by the summand of the variation of the bulk extension sourced by the gauge transformation of the connection 1-form A that is proportional to $\mathrm{d} \Lambda$, and the penultimate one represents the infinitesimal transformation of the gauge field in the defect extension. Finally, the contribution from the defect junctions is the boundary term of the integration by parts of the gauge-field variation in the defect extension. Using the arbitrariness of the embedding map $\varphi$ and that of the gauge potential A, we may next set to zero separately the terms independent of and linear in A, whereupon we obtain the following set of constraints:

$$
\begin{gathered}
\imath_{a} \omega+\Delta_{Q} \kappa_{a}+\mathrm{d} \gamma_{a}=0, \quad \Delta_{T_{n_{\jmath}}} \gamma_{a}=0 \\
\mathscr{L}_{a} \gamma_{b}=f_{a b c} \gamma_{c}
\end{gathered}
$$

which proves the claim.

We are thus led to the conclusion 
Corollary 3.11. In the notation of Proposition 3.10, the formula

$$
\widehat{\mathscr{A}_{\mathrm{WZ}}}[(\varphi \mid \Gamma) ; \mathrm{A}]=\mathrm{e}^{\mathrm{i} \int_{\Sigma} \phi^{*} \rho_{\mathrm{A}}} \mathrm{e}^{\mathrm{i} \int_{\Gamma}\left(\left.\phi\right|_{\Gamma}\right)^{*} \lambda_{\mathrm{A}}} \mathscr{A}_{\mathrm{WZ}}(\varphi \mid \Gamma)
$$

with $\rho_{\mathrm{A}}$ and $\lambda_{\mathrm{A}}$ given by Eq.(3.15) and Eq. (3.16), respectively, defines an infinitesimally gauge-invariant extension of $\mathscr{A}_{\mathrm{WZ}}(\varphi \mid \Gamma)$ in the presence of a defect quiver $\Gamma$ embedded in $\Sigma$ iff the 1-forms $\kappa_{a}$, introduced by Eq. (2.27), and the functions $k_{a}$, defined by Eqs. (2.28)(2.29), satisfy conditions (3.6)-(3.8). In other words, the latter conditions assure the absence of local gauge anomalies in the amplitudes (3.26).

Similarly to the defect-free case, the new conditions for a consistent gauging admit a simple cohomological, canonical, gerbe-theoretic and generalised-geometric interpretation. As previously, we focus on the first two, relegating the latter two to Sections 6 and 7 .

Remark 3.12. The constraints arising in the presence of boundary defects (equivalent to boundaries of an open world-sheet) were rephrased in the language of the g-equivariant cohomology of the world-volume $Q$ of the corresponding boundary bi-brane (or D-brane) by Figueroa-O'Farrill and Mohammedi in Ref. [FOM05]. Here, we extend their results to arbitrary defect quivers embedded in a closed world-sheet.

Proposition 3.13. Let $\mathscr{F}=M \sqcup Q \sqcup T$ be a $\mathrm{G}$-space satisfying the assumptions of Proposition 2.21, and let $\omega$ be a smooth G-invariant 2-form on $Q$ obeying relations (2.3) and (2.4), the former with respect to a 3-form $\mathrm{H}$ on $M$ with properties listed in Proposition 3.6. Relations (2.28)-(2.29) and (3.7) are satisfied iff $\omega$ admits a $\mathfrak{g}$-equivariant extension

$$
\widehat{\omega}=\omega-k, \quad k \in \Omega^{0}(Q) \otimes \mathfrak{g}^{*},
$$

given in terms of a $\mathfrak{g}$-equivariant 0 -form $k=k_{a} \otimes \tau^{a}$ in the Cartan model of the $\mathfrak{g}$-equivariant cohomology, that satisfies the identities

$$
\begin{gathered}
\widehat{\mathrm{d}} \widehat{\omega}=-\Delta_{Q} \widehat{\mathrm{H}}, \\
\Delta_{T_{n}} \widehat{\omega}=0 .
\end{gathered}
$$

Proof. Obvious, through inspection.

In the Weil model of the $\mathfrak{g}$-equivariant cohomology, we find

Proposition 3.14. In the notation of Proposition 3.7 and under the assumptions of Proposition 3.13, the Weil transform $\omega_{\mathrm{A}}=\mathrm{e}^{-\overline{\mathrm{A}_{1 *}}} \cdot \widehat{\omega}_{2^{*}}\left(\mathrm{~F}_{1^{*}}\right)$ of $\widehat{\omega}$ reads

$$
\omega_{\mathrm{A}}=\omega_{2 *}-\underline{\Delta}_{Q} \rho_{\mathrm{A}}+\mathrm{d} \lambda_{\mathrm{A}} \text {. }
$$

It satisfies the identities

$$
\mathrm{d} \omega_{\mathrm{A}}=-\underline{\Delta}_{Q} \mathrm{H}_{\mathrm{A}}, \quad \underline{\Delta}_{T_{n}} \omega_{\mathrm{A}}=0,
$$

written in terms of $\Delta_{T_{n}}=\sum_{k=1}^{n} \varepsilon_{n}^{k, k+1}\left(\operatorname{id}_{\mathfrak{V}_{\Gamma}} \times \pi_{n}^{k, k+1}\right)^{*}$.

Proof. Obvious, through inspection.

Remark 3.15. The canonical description of the $\sigma$-model in the presence of conformal defects was set up in Ref. Sus11, and its symmetries were subsequently studied at length in Ref. Sus12]. The analysis yields

Proposition 3.16. Sus12, Props. 3.10 \& 5.11] The symplectic realisation, mentioned in Proposition 2.28, of the symmetry algebra $\mathfrak{g}$ on the phase space $\mathrm{P}_{\sigma}$ of the non-linear twodimensional $\sigma$-model on a lorentzian world-sheet with an embedded defect quiver is hamiltonian iff conditions (3.6) and (3.7) are satisfied.

One can also interpret relation 2.29) as an intertwiner condition for symmetry-generating hamiltonians on twisted multi-string phase spaces, cf. Ref. [Sus12, Thm. 6.4].

The hitherto findings can be phrased succinctly as 
Corollary 3.17. The Feynman amplitudes of the non-linear $\sigma$-model on a closed oriented world-sheet $(\Sigma, \gamma)$, with an embedded defect quiver $\Gamma$, coupled to the topologically trivial gauge field $\mathrm{A}$ on $\Sigma$ take the form

$$
\mathscr{A}[(\varphi \mid \Gamma) ; \mathrm{A}, \gamma]=\exp \left[-\frac{1}{2} \int_{\Sigma} \mathrm{g}_{\mathrm{A}}\left(\mathrm{d} \phi \hat{,} \star_{\gamma} \mathrm{d} \phi\right)\right] \operatorname{Hol}_{\mathcal{G}_{\mathrm{A}}, \Phi_{\mathrm{A}},\left(\varphi_{n \mathrm{~A}}\right)}(\phi \mid \Gamma),
$$

where $\phi=\left(\mathrm{id}_{\Sigma}, \varphi\right)$, and the extended string background $\mathfrak{B}_{\mathrm{A}}=\left(\mathcal{M}_{\mathrm{A}}, \mathcal{B}_{\mathrm{A}}, \mathcal{J}_{\mathrm{A}}\right)$ defining the holonomy has components:

- the extended target $\mathcal{M}_{\mathrm{A}}$ composed of the target space $\Sigma \times M$ with metric $\mathrm{g}_{\mathrm{A}}$ of Eq. (3.17) and gerbe $\mathcal{G}_{\mathrm{A}}=\mathcal{G}_{2} * I_{\rho_{\mathrm{A}}}$, with $\rho_{\mathrm{A}}$ as in Eq. (3.15), of curvature $\mathrm{H}_{\mathrm{A}}$ given by Eq.(3.22);

- the extended bi-brane $\mathcal{B}_{\mathrm{A}}$ with world-volume $\Gamma \times Q$, bi-brane maps $\iota_{\alpha}=\mathrm{id}_{\Gamma} \times \iota_{\alpha}, \alpha=$ 1,2 , curvature $\omega_{\mathrm{A}}$ given by Eq. (3.29), and the 1-isomorphism $\Phi_{\mathrm{A}}=\Phi_{2^{*}} \otimes J_{\lambda_{\mathrm{A}}}$, where $\lambda_{\mathrm{A}}$ is as in Eq. (3.16);

- the extended inter-bi-brane $\mathcal{J}_{\mathrm{A}}$ with component world-volumes $\mathfrak{V}_{\Gamma}^{(n)} \times T_{n}, n \geq 3$, defined in terms of the subsets $\mathfrak{V}_{\Gamma}^{(n)} \subset \mathfrak{V}_{\Gamma}$ composed of vertices of valence $n$, with inter-bi-brane maps $\underline{\pi}_{n}^{k, k+1}=\mathrm{id}_{\mathfrak{V}_{\Gamma}^{(n)}} \times \pi_{n}^{k, k+1}, k=1,2, \ldots, n$, and 2-isomorphisms $\varphi_{n \mathrm{~A}}:=\varphi_{n 2^{*}}$.

Amplitudes (3.30) are invariant under infinitesimal gauge transformations (3.20).

\section{LARGE GAUGE TRANSFORMATIONS IN THE TOPOLOGICALLY TRIVIAL SECTOR}

In the preceding sections, we consistently gauged rigid symmetries of the $\sigma$-model target in a way that renders the $\sigma$-model invariant under infinitesimal gauge transformations. Invariance under the latter ensures also invariance under the so-called 'small' gauge transformations $\chi: \Sigma \rightarrow \mathrm{G}$ that can be continuously deformed to the identity (and, thus, necessarily take values in the connected component of the group unit of G). For general G (in particular, whenever the group manifold is not connected or not simply connected, or both), we are still left with the task of incorporating large gauge transformations (not homotopic to the identity transformation) into the formalism developed above. To accommodate nonconnected groups, we shall assume that the $\mathfrak{g}^{*}$-valued 1-form $\kappa=\kappa_{a} \otimes \tau^{a}$ on $M$ and the $\mathfrak{g}^{*}$-valued function $k=k_{a} \otimes \tau^{a}$ on $Q$ are not only $\mathfrak{g}$-equivariant, as required by Eqs. (3.6) and (3.7), but G-equivariant. Let us carry out a detailed analysis of invariance properties of the gauged decorated-surface holonomy (the invariance of the minimally coupled 'metric' term has already been demonstrated in the proof of Proposition 3.1). We shall consider arbitrary group-valued gauge transformations $\chi \in C^{\infty}(\Sigma, \mathrm{G})$ (for the trivial principal G-bundle) that lift to the product space $\Sigma \times \mathscr{F}$ as in Eq. (3.14). The gauge transformations of the $\sigma$-model field and those of the gauge field are given by Eqs. (3.10) and (3.9), respectively. The attendant gauge transformations of the gauge-field strength read

$$
\mathrm{F} \mapsto \operatorname{Ad}_{\chi} \mathrm{F}=:{ }^{\chi} \mathrm{F} .
$$

The following auxiliary result will prove useful.

Lemma 4.1. The Weil transforms $\mathrm{H}_{\mathrm{A}} \in \Omega^{3}(\Sigma \times M)$ and $\omega_{\mathrm{A}} \in \Omega^{2}(\Gamma \times Q)$, defined in Eqs. (3.22) and (3.29), respectively, transform under $L_{\chi}$ of Eq.(3.14) as

$$
\begin{aligned}
L_{\chi}^{*} \mathrm{H}_{\mathrm{A}} & =\mathrm{H}_{\chi^{-1} \mathrm{~A}}, \\
L_{\chi}^{*} \omega_{\mathrm{A}} & =\omega_{\chi^{-1} \mathrm{~A}} .
\end{aligned}
$$

Proof. The first of the two relations, Eq. (4.2), was proven in Ref. GSW10, Lemma 4.1]. We shall therefore restrict ourselves to proving the second one. The proof is conceptually analogous to that of Eq. 4.2). Invoking Proposition 2.29, in conjunction with Eqs. (3.9) and (4.1), we find

$$
\begin{aligned}
& L_{\chi}^{*} \omega_{\mathrm{A}}(\sigma, q)=\omega_{\mathrm{A}}(\sigma, \chi(\sigma) \cdot q)=\left(\mathrm{e}^{-\overline{\chi^{*} \theta_{L}(\sigma)}} \cdot \ell_{\chi(\sigma)}^{*} \omega_{\mathrm{A}}(\sigma, \cdot)\right)(q) \\
& \equiv\left\{\mathrm{e}^{-\overline{\chi^{*} \theta_{L}(\sigma)}} \cdot \ell_{\chi(\sigma)}^{*} \mathrm{e}^{-\overline{\mathrm{A}(\sigma)}} \cdot[\omega-k(\mathrm{~F}(\sigma))]\right\}(q)=\left\{\mathrm{e}^{-\overline{\chi^{-1} \mathrm{~A}(\sigma)}} \cdot\left[\omega-k\left(\chi^{-1} \mathrm{~F}(\sigma)\right)\right]\right\}(q)
\end{aligned}
$$




$$
\equiv \omega_{\chi^{-1} \mathrm{~A}}(\sigma, q) .
$$

The significance of the above transformation laws stems from the following observations: In the defect-free case, the gauge invariance of the 'topological' term of the $\sigma$-model is equivalent to the equality between the transformed Wess-Zumino amplitude

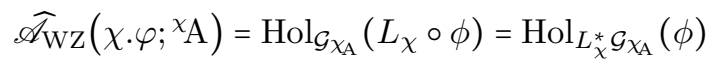

and the one before the transformation,

$$
\widehat{\mathscr{A}}_{\mathrm{WZ}}(\varphi ; \mathrm{A})=\operatorname{Hol}_{\mathcal{G}_{\mathrm{A}}}(\phi) .
$$

This leads us to compare the gerbes $L_{\chi}^{*} \mathcal{G}_{\chi_{\mathrm{A}}}$ and $\mathcal{G}_{\mathrm{A}}$ over $\Sigma \times M$, and Eq. 4.2), stating the equality of the respective curvatures, indicates that the two gerbes are related.

Proposition 4.2. GSW10, Prop. 4.2] Let $\mathcal{D}$ be the flat gerbe determined, alongside a canonical 1-isomorphism

$$
{ }^{M} \ell^{*} \mathcal{G} \stackrel{\cong}{\rightarrow} \mathcal{D} \otimes \mathcal{G}_{2^{*}} \otimes I_{\rho},
$$

by Eq. (2.39) and Proposition 2.8, and let $\mathcal{G}_{\mathrm{A}}$ be the gerbe defined in Corollary 3.17. Finally, let $L_{\chi}$ be the map given by Eq. (3.14). Then, the gerbes $L_{\chi}^{*} \mathcal{G}_{\chi_{\mathrm{A}}}$ and $\mathcal{G}_{\mathrm{A}} \otimes\left(\chi \times \operatorname{id}_{M}\right)^{*} \mathcal{D}$ are 1-isomorphic.

The last proposition, taken together with Eq. (4.4), readily implies

Theorem 4.3. GSW10, Thm. 4.3] Let $\widehat{\mathscr{A}}_{\mathrm{WZ}}(\varphi ; \mathrm{A})$ be the gauged Wess-Zumino amplitude of the defect-free $\sigma$-model, introduced in Corollary 3.17. Then, for any $\chi: \Sigma \rightarrow \mathrm{G}$, we have

$$
\widehat{\mathscr{A}}_{\mathrm{WZ}}\left(\chi \cdot \varphi ;{ }^{\chi} \mathrm{A}\right)=\widehat{\mathscr{A}}_{\mathrm{WZ}}(\varphi ; \mathrm{A}) \cdot \operatorname{Hol}_{\mathcal{D}}((\chi, \varphi)) \text {. }
$$

Taking, in identity (4.5), the gauge field $\mathrm{A}=\chi^{*} \theta_{L}$ for which ${ }^{\chi} \mathrm{A}=0$, one infers

Corollary 4.4. The holonomy of the flat gerbe $\mathcal{D}$ from Proposition 4.9 satisfies the identity

$$
\operatorname{Hol}_{\mathcal{D}}((\chi, \varphi))=\operatorname{Hol}_{\mathcal{G}}(\chi \cdot \varphi) \operatorname{Hol}_{\mathcal{G}}(\varphi)^{-1} \mathrm{e}^{-\mathrm{i} \int_{\Sigma} \phi^{*} \rho_{\chi^{*} \theta_{L}}} .
$$

The above relation was used in Ref. [GSW10] to identify the global gauge anomalies in the examples of gauged $\sigma$-models without defects, cf. also Section 5 below.

Theorem 4.3 has an important

Corollary 4.5. GSW10, Cor. 4.5] The gauged Wess-Zumino amplitude $\widehat{\mathscr{A}}_{\mathrm{WZ}}(\varphi ; \mathrm{A})$ of the defect-free $\sigma$-model, introduced in Corollary 3.17, is gauge invariant iff there exists a 1isomorphism

$$
\Upsilon:{ }^{M} \ell^{*} \mathcal{G} \stackrel{\cong}{\rightrightarrows} \mathcal{G}_{2^{*}} \otimes I_{\rho}
$$

for $\rho$ as in Eq. (2.30). It yields, in particular, the 1-isomorphism

$$
\left(\chi \times \operatorname{id}_{M}\right)^{*}: L_{\chi}^{*} \mathcal{G}_{\chi_{\mathrm{A}}} \cong \mathcal{G}_{\mathrm{A}} .
$$

We may next follow a similar reasoning in the case of a defect quiver with no junctions, $\mathfrak{V}_{\Gamma}=\varnothing$. Here, we compare

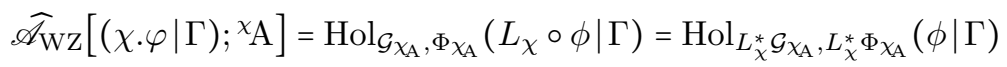

with

$$
\widehat{\mathscr{A}}_{\mathrm{WZ}}[(\varphi \mid \Gamma) ; \mathrm{A}]=\operatorname{Hol}_{\mathcal{G}_{\mathrm{A}}, \Phi_{\mathrm{A}}}(\phi \mid \Gamma) .
$$

The bulk analysis gives us the 1-isomorphism $\left(\chi \times \operatorname{id}_{M}\right)^{*} \Upsilon$, and so, in the light of Proposition 2.9, we are led to inspect the transformed bi-brane 1-isomorphism $L_{\chi}^{*} \mathcal{B}_{\chi \mathrm{A}}$. For this purpose, we decompose $L_{\chi}$, similarly as in Ref. GSW10, into

$$
\begin{aligned}
& L_{\chi}: \Gamma \times Q \stackrel{K_{\chi}}{\longrightarrow} \Gamma \times \mathrm{G} \times Q \stackrel{\mathrm{id}_{\Gamma} \times Q_{\ell}}{\longrightarrow} \Gamma \times Q \\
& (\sigma, q) \longmapsto(\sigma, \chi(\sigma), q) \longmapsto(\sigma, \chi(\sigma) . q)
\end{aligned}
$$


and compute

$$
\left(\operatorname{id}_{\Gamma} \times{ }^{Q} \ell\right)^{*} \Phi_{\mathrm{A}}=\left({ }^{Q} \ell^{*} \Phi\right)_{[2,3]^{*}} \otimes J\left(Q_{\ell^{*} \lambda}\right)_{\mathrm{A}},
$$

whereupon we take a closer look at the first factor of the tensor product. Consider the pair of gerbes

$$
{ }^{Q} \ell^{*} \iota_{1}^{*} \mathcal{G} \equiv \iota_{1}^{(1) * M} \ell^{*} \mathcal{G}, \quad \quad{ }^{Q} \ell^{*} \iota_{2}^{*} \mathcal{G} \otimes I_{Q_{\ell^{*} \omega}} \equiv \iota_{2}^{(1) * M} \ell^{*} \mathcal{G} \otimes I_{Q_{\ell^{*} \omega}}
$$

the equalities following from the assumed G-equivariance of the bi-brane maps. Recalling Eq. 2.41), we readily convince ourselves that both 1 -isomorphisms ${ }^{Q} \ell^{*} \Phi$ and $\left(\left(\iota_{2}^{(1) *} \Upsilon^{-1} \otimes\right.\right.$ $\left.\left.\operatorname{id}_{I_{Q_{\ell^{*} \omega \mathrm{d} \lambda}}}\right) \circ\left(\Phi_{2^{*}} \otimes \operatorname{id}_{I_{\iota_{1}^{(1) *}}}\right) \circ \iota_{1}^{(1) *} \Upsilon\right) \otimes J_{\lambda}$ map the first of the two gerbes in Eq. (4.10) into the second one. Thus, in virtue of Proposition 2.8, there exist a flat line bundle $D \stackrel{\pi_{D}}{\longrightarrow} \mathrm{G} \times Q$ and a 2-isomorphism

$$
\psi:{ }^{Q} \ell^{*} \Phi \stackrel{\cong}{\Longrightarrow} D \otimes\left(\left(\iota_{2}^{(1) *} \Upsilon^{-1} \otimes \mathrm{id}\right) \circ\left(\Phi_{2^{*}} \otimes \mathrm{id}\right) \circ \iota_{1}^{(1) *} \Upsilon\right) \otimes J_{\lambda}
$$

We are now ready to formulate the important

Proposition 4.6. Let $D$ and $\psi$ be the flat line bundle and the 2-isomorphism over $\mathrm{G} \times Q$ from Eq. (4.11), respectively, and let $\Phi_{\mathrm{A}}$ be the 1-isomorphism on $\Gamma \times Q$ defined in Corollary 3.17. Finally, let $L_{\chi}$ be the map given by Eq. (3.14). Then, we have the 2-isomorphism

$$
\begin{aligned}
& \left(\chi \times \operatorname{id}_{Q}\right)^{*} \psi \otimes \mathrm{id}: L_{\chi}^{*} \Phi \chi_{\mathrm{A}} \stackrel{\cong}{=} \\
& \quad\left(\chi \times \operatorname{id}_{Q}\right)^{*} D \otimes\left[\left(\underline{\iota}_{2}^{(1) *}\left(\chi \times \operatorname{id}_{M}\right)^{*} \Upsilon^{-1} \otimes \mathrm{id}\right) \circ\left(\Phi_{\mathrm{A}} \otimes \mathrm{id}\right) \circ \underline{\iota}_{1}^{(1) *}\left(\chi \times \operatorname{id}_{M}\right)^{*} \Upsilon\right],
\end{aligned}
$$

written in terms of the maps $\underline{\iota}_{\alpha}^{(1)}=\operatorname{id}_{\Gamma} \times \iota_{\alpha}, \alpha=1,2$.

Proof. Putting together Eqs. (4.9) and (4.11), as well as the identity

$$
\operatorname{pr}_{2,3} \circ K_{\chi}=\chi \times \operatorname{id}_{Q},
$$

we obtain

$$
\begin{gathered}
\left(\chi \times \operatorname{id}_{Q}\right)^{*} \psi \otimes \operatorname{id}_{K_{\chi}^{*} J}\left(Q_{\ell^{*} \lambda}\right)_{\chi_{\mathrm{A}}}=K_{\chi}^{*}\left(\psi_{[2,3]^{*}} \otimes \operatorname{id}_{J}\left(Q_{\ell^{*}}\right)_{\chi_{\mathrm{A}}}\right): L_{\chi}^{*} \Phi_{\chi_{\mathrm{A}}} \stackrel{\cong}{=} \\
\left(\chi \times \operatorname{id}_{Q}\right)^{*} D \otimes\left[\left(\left(\chi \times \operatorname{id}_{Q}\right)^{*} \iota_{2}^{(1) *} \Upsilon^{-1} \otimes \mathrm{id}\right) \circ\left(\Phi_{2^{*}} \otimes \mathrm{id}\right) \circ\left(\chi \times \operatorname{id}_{Q}\right)^{*} \iota_{1}^{(1) *} \Upsilon\right] \\
\otimes J\left(\chi \times \operatorname{id}_{Q}\right)^{*} \lambda+K_{\chi}^{*}\left(Q_{\ell^{*} \lambda}\right)_{\chi_{\mathrm{A}}} \\
=\left(\chi \times \operatorname{id}_{Q}\right)^{*} D \otimes\left[\left(\underline{(}_{2}^{(1) *}\left(\chi \times \operatorname{id}_{M}\right)^{*} \Upsilon^{-1} \otimes \mathrm{id}\right) \circ\left(\Phi_{2^{*}} \otimes \mathrm{id}\right) \circ \underline{\iota}_{1}^{(1) *}\left(\chi \times \operatorname{id}_{M}\right)^{*} \Upsilon\right] \\
\otimes J_{\left(\chi \times \operatorname{id}_{Q}\right)^{*} \lambda+L_{\chi}^{*} \lambda \chi_{\mathrm{A}} .}
\end{gathered}
$$

At this stage, it remains to verify the identity

$$
\left(\chi \times \operatorname{id}_{Q}\right)^{*} \lambda+L_{\chi}^{*} \lambda_{\chi_{\mathrm{A}}}=\lambda_{\mathrm{A}} .
$$

The latter follows upon summing up the right-hand sides of

$$
\left(\chi \times \operatorname{id}_{Q}\right)^{*} \lambda(\sigma, q)=\lambda(\chi(\sigma), q)=-k\left(\chi^{*} \theta_{L}(\sigma)\right)(q)
$$

and

$$
\begin{aligned}
& L_{\chi}^{*} \lambda_{\chi_{\mathrm{A}}}(\sigma, q) \\
& =\lambda_{\chi_{\mathrm{A}}}(\sigma, \chi(\sigma) \cdot q)=\left(\mathrm{e}^{-\overline{\chi^{*} \theta_{L}(\sigma)}} \cdot{ }^{Q} \ell_{\chi(\sigma)}^{*} \lambda \chi_{\mathrm{A}}(\sigma, \cdot)\right)(q) \equiv-\left[\mathrm{e}^{-\overline{\chi^{*} \theta_{L}(\sigma)}} \cdot{ }^{Q} \ell_{\chi(\sigma)}^{*} k\left({ }^{\chi_{\mathrm{A}}} \mathrm{A}(\sigma)\right)\right](q) \\
& =-k\left(\operatorname{Ad}_{\chi(\sigma)^{-1}}{ }^{\chi} \mathrm{A}(\sigma)\right)(q)=\lambda_{\mathrm{A}}(\sigma, q)+k\left(\chi^{*} \theta_{L}(\sigma)\right)(q),
\end{aligned}
$$

where in the last computation we used Proposition 2.29 and, subsequently, Eq. (3.9).

It is now straightforward to demonstrate the validity of 
Theorem 4.7. Let $\widehat{\mathscr{A}}_{\mathrm{Wz}}[(\varphi \mid \Gamma) ; \mathrm{A}]$ be the gauged Wess-Zumino amplitude, introduced in Corollary 3.17, of the $\sigma$-model for network-field configurations $(\varphi \mid \Gamma)$ for a defect quiver $\Gamma$ with no junctions. Suppose that there exists a 1-isomorphism $\Upsilon$ defined in Corollary 4.5, and let $D \rightarrow \mathrm{G} \times Q$ be the flat line bundle given in $E q$. (4.11). Then, for any $\chi: \Sigma \rightarrow \mathrm{G}$, we have

$$
\widehat{\mathscr{A}}_{\mathrm{WZ}}\left[(\chi \cdot \varphi \mid \Gamma) ;{ }^{\chi} \mathrm{A}\right]=\widehat{\mathscr{A}}_{\mathrm{WZ}}[(\varphi \mid \Gamma) ; \mathrm{A}] \cdot \operatorname{Hol}_{D}\left(\left.(\chi, \varphi)\right|_{\Gamma}\right) .
$$

Proof. Putting together Corollary 4.5, Proposition 4.6 and Proposition 2.9, we immediately conclude that the sole thing that remains to be proven is the equality between the standard line-bundle holonomy $\operatorname{Hol}_{D}\left(\left.(\chi, \varphi)\right|_{\Gamma}\right)$ and the holonomy-like defect contribution, as derived in Ref. [RS09, Sec. 2.7], of $\left(\chi \times \text { id }_{Q}\right)^{*} D$ to the gauge-transformed Wess-Zumino amplitude. To this end, we recall the explicit description of the inverse functor $\mathrm{Bun}^{-1}$ given in Ref. Wal07a, Sec. 2.5]. In the geometric language of the thesis, the latter assigns to the (flat) bundle $D$ over $\mathrm{G} \times Q$ the 1-isomorphism $I_{0} \rightarrow I_{0}$ defined by the trivial surjective submersion $\operatorname{id}_{\mathrm{G} \times Q}$, the same bundle $D$ over it, and the trivial isomorphism $\operatorname{id}_{D}$ as the bundle isomorphism compatible with the (trivial) groupoid structure on the fibres of the (trivial) bundle of $I_{0}$. The equality now follows by construction.

Taking, in identity (4.12), the gauge field $\mathrm{A}=\chi^{*} \theta_{L}$ for which ${ }^{\chi} \mathrm{A}=0$, one infers

Corollary 4.8. The holonomy of the flat line bundle D from Proposition 4.6 satisfies the identity

$$
\operatorname{Hol}_{D}\left(\left.(\chi, \varphi)\right|_{\Gamma}\right)=\operatorname{Hol}_{\mathcal{G}, \Phi}(\chi \cdot \varphi \mid \Gamma) \operatorname{Hol}_{\mathcal{G}, \Phi}(\varphi \mid \Gamma)^{-1} \mathrm{e}^{-\mathrm{i} \int_{\Sigma \backslash \Gamma} \phi^{*} \rho_{\chi^{*} \theta_{L}}-\mathrm{i} \int_{\Gamma}\left(\left.\phi\right|_{\Gamma}\right)^{*} \lambda_{\chi^{*} \theta_{L}}} .
$$

We shall use the above relation to identify the contribution to the global gauge anomaly from circular defects in examples of gauged $\sigma$-models considered in Section 5 .

Theorem 4.7 is at the basis of the following result:

Proposition 4.9. The gauged Wess-Zumino amplitude $\widehat{\mathscr{A}}_{\mathrm{WZ}}(\varphi ; \mathrm{A} \mid \Gamma)$ of the $\sigma$-model for network-field configurations $(\varphi \mid \Gamma)$ with a defect quiver $\Gamma$ without defect junctions is gauge invariant iff there exist: a 1-isomorphism

$$
\Upsilon:{ }^{M} \ell^{*} \mathcal{G} \stackrel{\cong}{\rightrightarrows} \mathcal{G}_{2^{*}} \otimes I_{\rho}
$$

with $\rho$ as in Eq. (2.30), and a 2-isomorphism

$$
\Xi:{ }^{Q} \ell^{*} \Phi \stackrel{\cong}{\Longrightarrow}\left(\left(\iota_{2}^{(1) *} \Upsilon^{-1} \otimes \mathrm{id}\right) \circ\left(\Phi_{2^{*}} \otimes \mathrm{id}\right) \circ \iota_{1}^{(1) *} \Upsilon\right) \otimes J_{\lambda}
$$

with $\lambda$ as in Eq. (2.31).

Proof. The holonomy, along $(\chi, \varphi)$, of the flat bundle $D$ over $\mathrm{G} \times Q$ that obstructs the desired equality of the Wess-Zumino amplitudes is trivial if $D$ is isomorphic to the trivial bundle $J_{0}$. The latter condition, by virtue of the definition of $D$, is equivalent to the existence of $\Xi$. This provides the proof of the "if" part of the proposition. A proof of its "only if" part is given in Appendix B.

Finally, we may consider a world-sheet with a general defect quiver. This time, we are to compare

$$
\widehat{\mathscr{A}}_{\mathrm{WZ}}\left[(\chi \cdot \varphi \mid \Gamma) ;{ }^{\chi} \mathrm{A}\right]=\operatorname{Hol}_{\mathcal{G}_{\chi_{A}}, \Phi_{\chi_{\mathrm{A}}},\left(\varphi_{n} \chi_{\mathrm{A}}\right)}\left(L_{\chi} \circ \phi \mid \Gamma\right)=\operatorname{Hol}_{L_{\chi}^{*} \mathcal{G}_{\chi_{\mathrm{A}}}, L_{\chi}^{*} \Phi \chi_{\mathrm{A}},\left(L_{\chi}^{*} \varphi_{n} \chi_{\mathrm{A}}\right)}(\phi \mid \Gamma)
$$

with

$$
\widehat{\mathscr{A}_{\mathrm{WZ}}}[(\varphi \mid \Gamma) ; \mathrm{A}]=\operatorname{Hol}_{\mathcal{G}_{\mathrm{A}}, \Phi_{\mathrm{A}},\left(\varphi_{n \mathrm{~A}}\right)}(\phi \mid \Gamma) .
$$

Upon decomposing the defect-junction restriction of $L_{\chi}$ as

$$
\begin{aligned}
L_{\chi}: \mathfrak{V}_{\Gamma}^{(n)} \times T_{n} & \stackrel{K_{\chi}}{\longrightarrow} \mathfrak{V}_{\Gamma}^{(n)} \times \mathrm{G} \times T_{n} \stackrel{\mathrm{id}_{\mathfrak{V}_{\Gamma}^{(n)}} \mathrm{x}^{T_{n}}}{\longrightarrow} \mathfrak{V}_{\Gamma}^{(n)} \times T_{n} \\
\left(\jmath, t_{n}\right) & \longmapsto\left(\jmath, \chi(\jmath), t_{n}\right) \stackrel{\longmapsto}{\longrightarrow}\left(\jmath, \chi(\jmath) \cdot t_{n}\right),
\end{aligned}
$$


we focus our attention on the 2-isomorphisms

$$
\left(\operatorname{id}_{\mathfrak{V}_{\Gamma}^{(n)}} \times{ }^{T_{n}} \ell\right)^{*} \varphi_{n \mathrm{~A}} \equiv\left(\operatorname{id}_{\mathfrak{V}_{\Gamma}^{(n)}} \times{ }^{T_{n}} \ell\right)^{*} \varphi_{n 2^{*}}=\left({ }^{T_{n}} \ell^{*} \varphi_{n}\right)_{[2,3]^{*}} .
$$

Reasoning along the same lines as in the case of non-intersecting defect lines, we infer, with the help of the identity

$$
\Delta_{T_{n}}^{(1)} \lambda=0
$$

implied by Eq. (2.29), and of Proposition 2.8, the equality of the 2-isomorphisms

$$
\begin{aligned}
T_{n} \ell^{*} \varphi_{n}= & d_{n} \otimes\left[\left(d_{\Upsilon}\right)_{n}^{1(1)} \bullet\left(\mathrm{id} \circ\left(i_{\Upsilon}^{\varepsilon_{n}^{1,2}}\right)_{n}^{1(1)}\right) \bullet\left(\mathrm{id} \circ \lambda_{\Upsilon_{n}^{1(1)}}\right) \bullet\left(\mathrm{id} \circ \varphi_{n} 2^{*} \circ \mathrm{id}\right)\right. \\
& \bullet\left(\mathrm{id} \circ \lambda_{\left(\Phi_{n}^{n, 1(1)}\right)_{2^{*}} \otimes \mathrm{id}} \circ \lambda_{\left(\Phi_{n}^{n-1, n}\right)_{2^{*}} \otimes \mathrm{id}} \circ \cdots \circ \lambda_{\left(\Phi_{n}^{2,3}\right)_{2^{*}} \otimes \mathrm{id}} \circ \mathrm{id}\right) \\
& \bullet\left(\operatorname{id} \circ\left(b_{\Upsilon}^{-1}\right)_{n}^{n(1)} \circ \mathrm{id} \circ\left(b_{\Upsilon}^{-1}\right)_{n}^{n-1(1)} \circ \mathrm{id} \circ \cdots \circ \mathrm{id} \circ\left(b_{\Upsilon}^{-1}\right)_{n}^{2(1)} \circ \mathrm{id}\right) \\
& \bullet\left(\mathrm{id} \circ\left(i_{\Upsilon}^{\varepsilon_{n}^{n, 1}}\right)_{n}^{n(1)} \circ \mathrm{id} \circ\left(i_{\Upsilon}^{\varepsilon_{n}^{n-1, n}}\right)_{n}^{n-1(1)} \circ \mathrm{id} \circ \cdots \circ \mathrm{id} \circ\left(i_{\Upsilon}^{\varepsilon_{n}^{2,3}}\right)_{n}^{2(1)} \circ \mathrm{id}\right) \\
& \left.\bullet\left(\left(\Xi_{n}^{n, 1(1)} \otimes \mathrm{id}\right) \circ\left(\Xi_{n}^{n-1, n(1)} \otimes \mathrm{id}\right) \circ \cdots \circ \Xi_{n}^{1,2(1)}\right)\right]
\end{aligned}
$$

for some locally constant $\mathrm{U}(1)$-valued functions $d_{n}$ on $\mathrm{G} \times T_{n}$. When writing the above, we used the maps

$$
\pi_{n}^{k(1)}:=\iota_{1}^{\varepsilon_{n}^{k, k+1}(1)} \circ \pi_{n}^{k, k+1(1)}
$$

the 1-isomorphism

$$
\Upsilon_{n}^{1(1)}:=\pi_{n}^{1(1) * \Upsilon}
$$

and the 2-isomorphisms

$$
\left(b_{\Upsilon}^{-1}\right)_{n}^{k(1)}:=\pi_{n}^{k(1) *} b_{\Upsilon}^{-1}, \quad\left(d_{\Upsilon}\right)_{n}^{k(1)}:=\pi_{n}^{k(1) *} d_{\Upsilon}
$$

as well as

$$
\left(i_{\Upsilon}^{\varepsilon_{n}^{k, k+1}}\right)_{n}^{k(1)}:=\pi_{n}^{k(1) *} i_{\Upsilon}^{\varepsilon_{n}^{k, k+1}}, \quad \quad i_{\Upsilon}^{\varepsilon_{n}^{k, k+1}}:=\left\{\begin{array}{l}
\operatorname{id}_{\Upsilon} \quad \text { if } \\
i_{\Upsilon} \text { otherwise }
\end{array} \varepsilon_{n}^{k, k+1}=1,\right.
$$

and

$$
\Xi_{n}^{k, k+1(1)}:=\pi_{n}^{k, k+1(1) *} \Xi^{\varepsilon_{n}^{k, k+1}}, \quad \Xi^{\varepsilon_{n}^{k, k+1}}:=\left\{\begin{array}{lc}
\Xi & \text { if } \\
\Xi^{\sharp-1} & \text { otherwise. }
\end{array} \varepsilon_{n}^{k, k+1}=1,\right.
$$

We thus obtain

Proposition 4.10. Let $d_{n}$ be the locally constant maps on the $\mathrm{G} \times T_{n}$ from Eq. 4.16), and let $\varphi_{n \mathrm{~A}}$ be the 2-isomorphism on $\mathfrak{V}_{\Gamma}^{(n)} \times T_{n}$ defined in Corollary 3.17. Finally, let $L_{\chi}$ be the map given by Eq. (3.14). Then, we have the equality

$$
\begin{aligned}
& L_{\chi}^{*} \varphi_{n} \chi_{\mathrm{A}} \\
& =\left(\chi, \operatorname{id}_{T_{n}}\right)^{*} d_{n} \otimes\left\{\left(d_{\left(\chi \times \mathrm{id}_{M}\right)^{*} \Upsilon}\right)_{n}^{\frac{1}{n}} \bullet\left(\mathrm{id} \circ\left(i_{\Upsilon}^{\varepsilon_{n}^{1,2}}\right)_{n}^{\frac{1}{n}}\right) \bullet\left(\mathrm{id} \circ \lambda_{\left(\left(\chi \times \mathrm{id}_{M}\right) * \Upsilon\right) \frac{1}{n}}\right) \bullet\left(\mathrm{id} \circ \varphi_{n \mathrm{~A}} \circ \mathrm{id}\right)\right. \\
& \bullet\left(\operatorname{id} \circ \lambda_{\left(\Phi_{n}^{n, 1}\right)_{2^{*}} \otimes \mathrm{id}} \circ \lambda_{\left(\Phi_{n}^{n-1, n}\right)_{2^{*}} \otimes \mathrm{id}} \circ \cdots \circ \lambda_{\left(\Phi_{n}^{2,3}\right)_{2^{*}} \otimes \mathrm{id}} \circ \mathrm{id}\right) \\
& \bullet\left(\operatorname{id} \circ\left(b_{\left(\chi \times \mathrm{id}_{M}\right) * \Upsilon}^{-1}\right)_{n}^{\frac{n}{n}} \circ \mathrm{id} \circ\left(b_{\left(\chi \times \mathrm{id}_{M}\right)^{*}}^{-1}\right)_{n}^{\frac{n-1}{n}} \circ \mathrm{id} \circ \cdots \circ \mathrm{id} \circ\left(b_{\left(\chi \times \mathrm{id}_{M}\right)^{*} \Upsilon}^{-1}\right)_{n}^{\frac{2}{n}} \circ \mathrm{id}\right) \\
& \bullet\left(\operatorname{id} \circ\left(i_{\left(\chi \times \operatorname{id}_{M}\right) * \Upsilon}^{\varepsilon_{n}^{n, 1}}\right)_{n}^{\frac{n}{n}} \circ \mathrm{id} \circ\left(i_{\left(\chi \times \operatorname{id}_{M}\right) * \Upsilon}^{\varepsilon_{n}^{n-1, n}}\right) \frac{n-1}{n} \circ \mathrm{id} \circ \cdots \circ \mathrm{id} \circ\left(i_{\left(\chi \times \operatorname{id}_{M}\right) * \Upsilon}^{\varepsilon_{n}^{2,3}}\right)_{n}^{\frac{2}{n}} \circ \mathrm{id}\right) \\
& \left.\bullet\left[\left[\left(\left(\chi \times \operatorname{id}_{Q}\right)^{*} \Xi\right) \frac{n, 1}{n} \otimes \mathrm{id}\right] \circ\left[\left(\left(\chi \times \operatorname{id}_{Q}\right)^{*} \Xi\right) \frac{n-1, n}{n} \otimes \mathrm{id}\right] \circ \cdots \circ\left(\left(\chi \times \operatorname{id}_{Q}\right)^{*} \Xi\right) \frac{1,2}{n}\right]\right\},
\end{aligned}
$$

written in terms of the maps

$$
\underline{\pi}_{n}^{k}:=\underline{\iota}_{1}^{\varepsilon_{n}^{k, k+1}} \circ \underline{\pi}_{n}^{k, k+1}
$$


the 1-isomorphisms

$$
\Upsilon \frac{k}{n}:=\underline{\pi}_{n}^{k *} \Upsilon
$$

and the 2-isomorphisms

$$
\left(b_{\Upsilon}^{-1}\right)_{n}^{\underline{k}}:=\underline{\pi}_{n}^{k *} b_{\Upsilon}^{-1}, \quad(d \Upsilon)_{n}^{\underline{k}}:=\underline{\pi}_{n}^{k *} d \Upsilon
$$

and

$$
\left(i_{\Upsilon}^{\varepsilon_{n}^{k, k+1}}\right)_{n}^{\underline{k}}:=\underline{\pi}_{n}^{k *} i_{\Upsilon}^{\varepsilon_{n}^{k, k+1}}, \quad \quad \Xi \frac{k, k+1}{n}:=\underline{\pi}_{n}^{k, k+1 *} \Xi^{\varepsilon_{n}^{k, k+1}},
$$

with the $i_{\Upsilon}^{\varepsilon_{n}^{k, k+1}}$ and $\Xi^{\varepsilon_{n}^{k, k+1}}$ as in Eqs. 4.18) and (4.19), respectively.

Proof. The proof is a simple exercise using the assumed G-equivariance of the (inter-)bi-brane maps.

The above proposition immediately yields

Theorem 4.11. Let $\widehat{\mathscr{A}}_{\mathrm{WZ}}[(\varphi \mid \Gamma) ; \mathrm{A}]$ be the gauged Wess-Zumino amplitude, introduced in Corollary 3.11, of the $\sigma$-model for network-field configurations $(\varphi \mid \Gamma)$ for an arbitrary defect quiver $\Gamma$. Suppose that there exist a 1-isomorphism $\Upsilon$ defined in Corollary 4.5 and a 2isomorphism $\Xi$ defined in Proposition 4.9. Finally, let $d_{n}$ be the locally constant $\mathrm{U}(1)$-valued functions on $\mathrm{G} \times T_{n}$ introduced in Eq.(4.16). Then, for any $\chi: \Sigma \rightarrow \mathrm{G}$, we have

$$
\widehat{\mathscr{A}}_{\mathrm{WZ}}\left[(\chi \cdot \varphi \mid \Gamma) ;{ }^{\chi} \mathrm{A}\right]=\widehat{\mathscr{A}}_{\mathrm{WZ}}[(\varphi \mid \Gamma) ; \mathrm{A}] \cdot \prod_{\jmath \in \mathfrak{V}_{\Gamma}}(\chi, \varphi)^{*} d_{n_{\jmath}}(\jmath)^{ \pm 1} .
$$

where the exponent +1 is taken for the positive defect junctions and -1 for the negative ones.

Proof. An obvious consequence of Corollary 4.5 and Proposition 4.9, taken in conjunction with Proposition 4.10.

Similarly as before, taking $\mathrm{A}=\chi^{*} \theta_{L}$, we infer from identity 4.20 ) that, under the assumptions of the above theorem,

$$
\begin{aligned}
\prod_{\jmath \in \mathfrak{V}_{\Gamma}}(\chi, \varphi)^{*} d_{n_{\jmath}}(\jmath)^{ \pm 1}= & \operatorname{Hol}_{\mathcal{G}, \Phi,\left(\varphi_{n}\right)}(\chi \cdot(\varphi \mid \Gamma)) \operatorname{Hol}_{\mathcal{G}, \Phi,\left(\varphi_{n}\right)}(\varphi \mid \Gamma)^{-1} \\
& \cdot \mathrm{e}^{-\mathrm{i} \int_{\Sigma \backslash \Gamma} \phi^{*} \rho_{\chi^{*} \theta_{L}}-\mathrm{i} \int_{\Gamma}\left(\left.\phi\right|_{\Gamma}\right)^{*} \lambda_{\chi^{*} \theta_{L}}}
\end{aligned}
$$

Theorem 4.11 permits to obtain the following result that establishes necessary and sufficient conditions for gauge invariance of the gauged Wess-Zumino amplitudes with general networkfield configurations:

Theorem 4.12. The gauged Wess-Zumino amplitudes $\widehat{\mathscr{A}}_{\mathrm{WZ}}(\varphi ; \mathrm{A} \mid \Gamma)$, introduced in Corollary 3.11, are gauge invariant under all gauge transformations iff there exist: a 1-isomorphism

$$
\Upsilon:{ }^{M} \ell^{*} \mathcal{G} \stackrel{\cong}{\rightarrow} \mathcal{G}_{2 *} \otimes I_{\rho}
$$

for $\rho$ as in Eq. (2.30), and a 2-isomorphism

$$
\Xi:{ }^{Q} \ell^{*} \Phi \stackrel{\cong}{\Longrightarrow}\left(\left(\iota_{2}^{(1) *} \Upsilon^{-1} \otimes \mathrm{id}\right) \circ\left(\Phi_{2^{*}} \otimes \mathrm{id}\right) \circ \iota_{1}^{(1) *} \Upsilon\right) \otimes J_{\lambda},
$$

for $\lambda$ as in Eq.(2.31), such that the identities

$$
\begin{aligned}
T_{n} \ell^{*} \varphi_{n}= & (d \Upsilon)_{n}^{1(1)} \bullet\left(\mathrm{id} \circ\left(i_{\Upsilon}^{\varepsilon_{n}^{1,2}}\right)_{n}^{1(1)}\right) \bullet\left(\mathrm{id} \circ \lambda_{\Upsilon_{n}^{1(1)}}\right) \bullet\left(\mathrm{id} \circ \varphi_{n} 2^{*} \circ \mathrm{id}\right) \\
& \bullet\left(\operatorname{id} \circ \lambda_{\left(\Phi_{n}^{n, 1(1)}\right)_{2^{*}} \otimes \mathrm{id}} \circ \lambda_{\left(\Phi_{n}^{n-1, n}\right)_{2^{*}} \otimes \mathrm{id}} \circ \cdots \circ \lambda_{\left(\Phi_{n}^{2,3}\right)_{2^{*}} \otimes \mathrm{id}} \circ \mathrm{id}\right) \\
& \bullet\left(\operatorname{id} \circ\left(b_{\Upsilon}^{-1}\right)_{n}^{n(1)} \circ \mathrm{id} \circ\left(b_{\Upsilon}^{-1}\right)_{n}^{n-1(1)} \circ \mathrm{id} \circ \cdots \circ \mathrm{id} \circ\left(b_{\Upsilon}^{-1}\right)_{n}^{2(1)} \circ \mathrm{id}\right) \\
& \bullet\left(\mathrm{id} \circ\left(i_{\Upsilon}^{\varepsilon_{n}^{n, 1}}\right)_{n}^{n(1)} \circ \mathrm{id} \circ\left(i_{\Upsilon}^{\varepsilon_{n}^{n-1, n}}\right)_{n}^{n-1(1)} \circ \mathrm{id} \circ \cdots \circ \mathrm{id} \circ\left(i_{\Upsilon}^{\varepsilon_{n}^{2,3}}\right)_{n}^{2(1)} \circ \mathrm{id}\right) \\
& \bullet\left(\left(\Xi_{n}^{n, 1(1)} \otimes \mathrm{id}\right) \circ\left(\Xi_{n}^{n-1, n(1)} \otimes \mathrm{id}\right) \circ \cdots \circ \Xi_{n}^{1,2(1)}\right)
\end{aligned}
$$

hold true for all $n \geq 3$. 
Proof. If the conditions listed above are satisfied then the locally constant functions $d_{n}$ on $\mathrm{G} \times T_{n}$ introduced by (4.16) are equal to 1 and the gauge invariance of the amplitudes follows from Theorem 4.11. A proof of the "only if" part of the theorem is given in Appendix B.

Remark 4.13. In particular, the conditions listed in Theorem 4.12 assure the absence of global gauge anomalies in amplitudes (3.26).

\section{An example of a String BaCkground: the WZW MOdel}

5.1. The WZW target. We present here in some detail a distinguished class of string backgrounds, with all components given by subspaces of the group manifold of some connected compact semi-simple Lie group $\mathscr{G}$, not necessarily simply connected, endowed with additional cohomological structure. These are the backgrounds in which the lagrangian fields of the so-called Wess-Zumino-Witten (WZW) $\sigma$-model of Ref. Wit84 take values. One has: $\mathscr{G}=\tilde{\mathrm{G}} / Z$, where $\tilde{\mathrm{G}}=\times_{l} \tilde{\mathrm{G}}_{l}$ is the covering group of $\mathscr{G}$ that decomposes into the product of simple factors, and $Z$ is a subgroup of the center $\tilde{Z}=\times_{l} \tilde{Z}_{l}$ of $\tilde{\mathrm{G}}$. The Lie algebra $\mathfrak{g}$ of $\tilde{\mathrm{G}}$ decomposes as $\oplus_{l} \mathfrak{g}_{l}$ into the direct sum of simple factors. Let $\mathrm{G}=\tilde{\mathrm{G}} / \tilde{Z}$. We shall consider $\mathscr{G}$ with the adjoint action of $\mathrm{G}$.

Let $\operatorname{ktr}_{\mathfrak{g}} X Y:=\sum \mathrm{k}_{l} \operatorname{tr}_{\mathfrak{g}_{l}} X^{l} Y^{l}$ be an $a d$-invariant negative-definite bilinear form on the Lie algebra $\mathfrak{g}$, given by the sum of forms on $\mathfrak{g}_{l}$ with the same properties, where $\mathrm{k}=\left(\mathrm{k}_{l}\right)$, with $\mathrm{k}_{l}>0$, is called the level. We shall consider the Cartan-Killing metric on $\mathscr{G}$ given by

$$
g_{k}=-\frac{1}{4 \pi} k \operatorname{tr}_{\mathfrak{g}}\left(\theta_{L} \otimes \theta_{L}\right)
$$

and the closed 3 -form

$$
\mathrm{H}_{\mathrm{k}}=\frac{1}{12 \pi} \mathrm{k} \operatorname{tr}_{\mathfrak{g}}\left(\theta_{L} \wedge \theta_{L} \wedge \theta_{L}\right),
$$

where $\theta_{L}$ stands for the left-invariant Maurer-Cartan form on $\mathscr{G}$. The bilinear forms $\operatorname{tr}_{\mathfrak{g}_{l}}$ on the simple Lie-algebra factors $\mathfrak{g}_{l}$ are assumed to be normalized so that if the group $\mathscr{G}$ is simply connected (i.e. $\mathscr{G}=\tilde{\mathrm{G}}$ ) then the periods of the 3 -form $\mathrm{H}_{\mathrm{k}}$ take values in $2 \pi \mathbb{Z}$ iff all $\mathrm{k}_{l}$ are integers. For non-simply connected $\mathscr{G}$, the integrality of the periods of $\frac{1}{2 \pi} \mathrm{H}_{\mathrm{k}}$ imposes more stringent selection rules on the level k, cf. Refs. [FGK88, GR03]. Such integrality is necessary and sufficient for the existence of a gerbe $\mathcal{G}_{\mathrm{k}}$ over group $\mathscr{G}$ with curvature 3form $\mathrm{H}_{\mathrm{k}}$ whose holonomy provides the Wess-Zumino part of Feynman amplitudes for the defect-free WZW $\sigma$-models of conformal field theory.

Definition 5.1. The level-k WZW target is the triple $\left(\mathscr{G}, g_{k}, \mathcal{G}_{\mathrm{k}}\right)$.

We shall consider the defect-free WZW model defined for the above target with rigid symmetries induced by the adjoint action of $\mathrm{G}$ on $\mathscr{G}$ (the other symmetries from the leftright symmetry group $\mathscr{G} \times \mathscr{G}$, that extend to the loop-group $L \mathscr{G} \times L \mathscr{G}$ symmetries of the defect-free WZW model, cannot be gauged as they suffer from local gauge anomalies). Choose generators $t_{a}, a=1,2, \ldots, \operatorname{dim} \mathfrak{g}$, of the Lie algebra $\mathfrak{g}$ as in Eq. (2.12). They induce on $\mathscr{G}$ the fundamental vector fields

$$
\mathscr{G}_{\mathscr{K}_{a}}=L_{a}-R_{a},
$$

where the $L_{a}$ (resp. $R_{a}$ ) are the left-invariant (resp. right-invariant) vector fields on $\mathscr{G}$ corresponding to the generators $t_{a} \in \mathfrak{g}$.

Proposition 5.2. The collection $\left\{v_{a}\right\}_{a=1,2, \ldots, \operatorname{dimg}}$ of 1 -forms on $\mathscr{G}$ given by

$$
v_{a}=-\frac{1}{4 \pi} \mathrm{k} \operatorname{tr}_{\mathfrak{g}}\left(t_{a}\left(\theta_{L}+\theta_{R}\right)\right)
$$

defines a $\mathfrak{g}$-equivariantly closed G-equivariant (Cartan-model) extension of the Cartan 3-form $\mathrm{H}_{\mathrm{k}}$ on $\mathscr{G}$, as defined in Eq. (5.1), through

$$
\widehat{\mathrm{H}}_{\mathrm{k}}=\mathrm{H}_{\mathrm{k}}+v, \quad v\left(t_{a}\right)=v_{a} .
$$

Proof. Obvious, through inspection.

\footnotetext{
${ }^{1}$ One could extend the discussion below to the setting of not necessarily compact Lie groups.
} 
The last proposition lays out the basic setting for the gauging of (continuous) rigid symmetries of the WZW model?

5.2. The boundary maximally symmetric WZW bi-branes. In the next step, we consider $\mathcal{G}_{\mathrm{k}}$-bi-branes for those boundary WZW defects that preserve a maximum amount of the loop-group symmetry $\mathrm{L} \mathscr{G} \times \mathrm{L} \mathscr{G}$ of the WZW model in the interior of the world-sheet, that is a single copy of $L \mathscr{G}$ embedded 'diagonally' in the double cartesian product. As argued in Remark 2.5 (and originally in Ref. [RS09, p. 12]), a consistent description of a $\sigma$-model on a world-sheet with a boundary in the language of defects and bi-branes assigned to them prerequires a special choice of the target, which - in the case in hand - is simply the disjoint union of the level-k WZW target group $\mathscr{G}$ with an arbitrary singleton $\{\bullet\}$ with trivial geometric data over it. By a slight abuse of the language, we shall refer also to this target as the level-k WZW target in what follows.

Definition 5.3. A boundary maximally symmetric $\mathcal{G}_{\mathrm{k}}$-bi-brane, or a maximally symmetric $\mathcal{G}_{\mathrm{k}}$-brane for short, is a quintuple $\mathcal{B}_{\lambda}^{\partial}:=\left(\mathscr{C}_{\lambda}, \iota_{\lambda}, \bullet, \omega_{\lambda}^{\partial}, \mathcal{T}_{\lambda}\right)$ with the following components:

(D.1) the world-volume given by the conjugacy class

$$
\mathscr{C}_{\lambda}=\left\{\operatorname{Ad}_{x}\left(t_{\lambda}\right) \mid x \in \mathscr{G}\right\},
$$

of an element $t_{\lambda}=\mathrm{e}^{2 \pi \lambda} \in \mathscr{G}$ of the Cartan subgroup of $\mathscr{G}$, with $\lambda=\oplus_{l} \lambda_{l}$ from the fundamental affine Weyl alcove[,

$$
\mathscr{A}_{W}(\mathfrak{g})=\left\{\lambda \in \mathfrak{t} \quad \mid \operatorname{tr}_{\mathfrak{g}}(\lambda \vartheta) \leq 1, \quad \operatorname{tr}_{\mathfrak{g}}\left(\lambda \alpha_{i}\right) \geq 0, i=1,2, \ldots, \operatorname{rank} \mathfrak{g}\right\},
$$

of the Cartan subalgebra $\mathfrak{t}=\oplus_{l} \mathfrak{t}_{l} \subset \mathfrak{g}$ defined in terms of the simple roots $\alpha_{i}$ of $\mathfrak{g}$ and its highest root $\vartheta$;

(D.2) the embedding $\iota_{\lambda}: \mathscr{C}_{\lambda} \hookrightarrow \mathscr{G}$ of the conjugacy class in the group manifold, alongside the constant map $\bullet: \mathscr{C}_{\lambda} \rightarrow\{\bullet\}$

(D.3) the curvature 2 -form

$$
\omega_{\lambda}^{\partial}=\frac{1}{8 \pi} k \operatorname{tr}_{\mathfrak{g}}\left(\theta_{L} \wedge \frac{\mathrm{id}_{\mathfrak{g}}+\mathrm{Ad}_{\mathfrak{g}}}{\mathrm{id}_{\mathfrak{g}}-\mathrm{Ad}_{\bullet}} \theta_{L}\right)
$$

providing a global primitive for $\iota_{\lambda}^{*} \mathrm{H}_{\mathrm{k}}$;

(D.4) the 1 -isomorphism given by a trivialisation $\mathcal{T}_{\lambda}: \iota_{\lambda}^{*} \mathcal{G}_{\mathrm{k}} \stackrel{\cong}{\rightarrow} I_{\omega_{\lambda}^{\partial}}$ of gerbe $\mathcal{G}_{\mathrm{k}}$ restricted to $\mathscr{C}_{\lambda}$.

Remark 5.4. It is vital to note that for an arbitrary compact simple 1-connected Lie group $\mathscr{G}$ trivializations $\mathcal{T}_{\lambda}$ exist iff

$$
\mathrm{k} \lambda:=\oplus_{l} \mathrm{k}_{l} \lambda_{l} \in \mathrm{k} \mathscr{A}_{W}(\mathfrak{g}) \cap P(\mathfrak{g})=: P_{+}^{\mathrm{k}}(\mathfrak{g})
$$

where $P(\mathfrak{g})$ denotes the weight lattice of $\mathfrak{g}$, whereas for non-simply connected $\mathrm{G}$ additional selection rules may be required for $\lambda$, cf. Ref. [Gaw05]. In the first case, the conjugacy classes $\mathscr{C}_{\lambda}$, each corresponding to a unique $\lambda \in \mathscr{A}_{W}(\mathfrak{g})$, are 1 -connected, whereas in the second case, they are connected but not necessarily simply connected, and different $\lambda \in \mathscr{A}_{W}(\mathfrak{g})$ may correspond to the same $\mathscr{C}_{\lambda}$.

The world-volume of a maximally symmetric WZW $\mathcal{G}_{\mathrm{k}}$-brane is naturally endowed with the structure of a G-space coming from the adjoint action of $\mathrm{G}$ on $\mathscr{G}$.

Proposition 5.5. Adopt the notation of Definition 5.1 and Proposition 5.9, and let $\mathscr{C}_{\lambda}$ be a conjugacy class in $\mathscr{G}$, as defined in Eq. (5.3). The 2-form $\omega_{\lambda}^{\partial}$ on $\mathscr{C}_{\lambda}$ given in Eq. (5.4) is its own (trivial) $\mathscr{G}$-equivariant (Cartan-model) extension satisfying Eq. (3.27) with respect to the Cartan 3-form $\mathrm{H}_{\mathrm{k}}$ on $\mathscr{G}$, and for the pair of $\mathrm{G}$-equivariant maps $\left(\iota_{\lambda}, \bullet\right)$ given in Definition 5.5 .

Proof. Through inspection.

\footnotetext{
${ }^{2}$ Another class of examples of constructions considered in this paper is provided by the orbifold WZW models of Refs. GR02, GR03, Gaw05, cf. also Refs. GSW08b, GSW08a for a generalisation to WZW orientifolds.

${ }^{3}$ In what follows, we shall always identify $\mathfrak{t}$ with its dual $\mathfrak{t}^{\star}$ using the bilinear form $\operatorname{tr}_{\mathfrak{g}}$ on $\mathfrak{g}$.
} 
Definition 5.6. A general boundary maximally symmetric $\mathcal{G}_{\mathrm{k}}$-bi-brane is a disjoint union of the elementary boundary maximally symmetric $\mathcal{G}_{\mathrm{k}}$-bi-branes of Definition 5.3 .

5.3. The non-boundary maximally symmetric WZW bi-branes. As the last example of WZW $\mathcal{G}_{\mathrm{k}}$-bi-branes, we present those associated with non-boundary WZW defects that preserve the full loop-group symmetry $\mathrm{L} \mathscr{G} \times \mathrm{L} \mathscr{G}$ of the WZW model. They implement jumps by elements of the target Lie group in the sense that the limiting values attained by the onesided local extensions $g_{\mid 1}$ (from the left) and $g_{\mid 2}$ (from the right) of the field $g: \Sigma \backslash \Gamma \rightarrow \mathscr{G}$ to the defect line $\Gamma$ (as given in Definition 2.4) are, in general, different. A special class of such defects, the central-jump defects for which $g_{\mid 1}^{-1} g_{\mid 2} \in Z$, where $Z$ is the center of $\mathscr{G}$, were considered at length in Ref. RS09. The more general jump defects, with the jump given by $g_{\mid 1}^{-1} \cdot g_{\mid 2} \in \mathscr{C}_{\lambda}$, were first considered in Ref. [FSW08], where the notion of a bi-brane was introduced. They will be discussed below, cf. also Ref. [RS11b] for more details.

Definition 5.7. A non-boundary maximally symmetric $\mathcal{G}_{\mathrm{k}}$-bi-brane is a quintuple $\mathcal{B}_{\lambda}:=\left(Q_{\lambda}, \operatorname{pr}_{1}, \mathrm{~m}, \omega_{\lambda}, \Phi_{\lambda}\right)$ with the following components:

(B.1) the world-volume

$$
Q_{\lambda}=\mathscr{G} \times \mathscr{C}_{\lambda}, \quad \lambda \in P_{+}^{\mathrm{k}}(\mathfrak{g}),
$$

isomorphic with the bi-conjugacy class

$$
\mathscr{B}_{\left(t_{\lambda}, e\right)}=\left\{\left(x \cdot t_{\lambda} \cdot y^{-1}, x \cdot y^{-1}\right) \quad \mid x, y \in \mathscr{G}\right\},
$$

of Ref. FSW08] through

$$
D: \mathscr{G} \times \mathscr{C}_{\lambda} \stackrel{\cong}{\rightarrow} \mathscr{B}_{\left(t_{\lambda}, e\right)}:\left(g, h_{\lambda}\right) \mapsto\left(g \cdot h_{\lambda}, g\right) ;
$$

(B.2) the canonical projection $\operatorname{pr}_{1}: Q_{\lambda} \rightarrow \mathscr{G}:\left(g, h_{\lambda}\right) \mapsto g$ and the multiplication map $\mathrm{m}: Q_{\lambda} \rightarrow \mathscr{G}:\left(g, h_{\lambda}\right) \mapsto g \cdot h_{\lambda} ;$

(B.3) the curvature 2 -form

$$
\omega_{\lambda}=-\omega_{\lambda 2^{*}}^{\partial}+\varrho_{\mathrm{k}}, \quad \varrho_{\mathrm{k}}=\frac{1}{4 \pi} \mathrm{k} \operatorname{tr}_{\mathfrak{g}}\left(\theta_{L 1^{*}} \wedge \theta_{R 2^{*}}\right),
$$

providing a global primitive for $\mathrm{H}_{\mathrm{k} 1^{*}}-\mathrm{m}^{*} \mathrm{H}_{\mathrm{k}}$;

(B.4) the 1-isomorphism $\Phi_{\lambda}=\left(\mathcal{M}_{\mathrm{k}} \otimes \mathrm{id}_{I_{-\omega_{\lambda}^{\partial}}}\right) \circ\left(\operatorname{id}_{\mathcal{G}_{k^{*}}} \otimes \mathcal{T}_{\lambda 2^{*}}^{-1} \otimes \operatorname{id}_{I_{-\omega_{\lambda}^{\partial}}}\right)$ (a gerbe bi-module) defined as

$$
\Phi_{\lambda}: \mathcal{G}_{\mathrm{k} 1^{*}} \equiv \mathcal{G}_{\mathrm{k} 1^{*}} \otimes I_{\omega_{\lambda}^{\partial}} \otimes I_{-\omega_{\lambda}^{\partial}} \stackrel{\cong}{\rightarrow} \mathrm{m}^{*} \mathcal{G}_{\mathrm{k}} \otimes I_{\omega_{\lambda}}
$$

in terms of a 1-isomorphism

$$
\mathcal{M}_{\mathrm{k}}: \mathcal{G}_{\mathrm{k} 1^{*}} \otimes \mathcal{G}_{\mathrm{k} 2^{*}} \stackrel{\cong}{\rightarrow} \mathrm{m}^{*} \mathcal{G}_{\mathrm{k}} \otimes I_{\varrho_{\mathrm{k}}}
$$

of the multiplicative structure on $\mathcal{G}_{\mathrm{k}}$, as introduced in Ref. [CJMSW05] and developed in Refs. Wal10, GW09.

Remark 5.8. It ought to be emphasised that in the case of simple 1-connected groups $\mathscr{G}$, the uniqueness (up to a 2 -isomorphism) of the 1-isomorphism $\mathcal{M}_{\mathrm{k}}$ ensures that nonboundary maximally symmetric WZW bi-branes are in a one-to-one correspondence with their boundary analogs.

We shall consider $Q_{\lambda}$ as a G-space with the diagonal adjoint action of $\mathrm{G}$.

Proposition 5.9. Adopt the notation of Definition 5.1 and Proposition 5.9, and let $\mathscr{C}_{\lambda}$ be a conjugacy class in $\mathscr{G}$, as defined in Eq. (5.3). The 2 -form $\omega_{\lambda}$ on $\mathscr{G}_{\times} \mathscr{C}_{\lambda}$ given in Eq. (5.5) is its own (trivial) $\mathscr{G}$-equivariant (Cartan-model) extension satisfying $E q$. (3.27) with respect to the Cartan 3-form $\mathrm{H}_{\mathrm{k}}$ on $\mathscr{G}$, as defined in Eq. (5.1), and for the pair of $\mathscr{G}$-equivariant maps $\iota_{1}:=\operatorname{pr}_{1}$ and $\iota_{2}:=\mathrm{m}$.

Proof. Through inspection.

Definition 5.10. A general non-boundary maximally symmetric $\mathcal{G}_{k}$-bi-brane is the disjoint union of the elementary non-boundary maximally symmetric $\mathcal{G}_{\mathrm{k}}$-bi-branes of Definition 5.7. 
5.4. The maximally symmetric WZW inter-bi-brane. Inter-bi-branes describe the behaviour of $\sigma$-model fields at junctions of defect lines. As such, they come in a countably infinite variety that encodes the various relative orientations (towards and away from the junction) of intersecting defect lines and the arbitrary valence of junctions. In what follows, we focus - for the sake of brevity - on the most elementary defect junctions, namely those with two incoming defect lines (i.e. oriented towards the junction) and a single outgoing defect line (i.e. oriented away from the junction). In the case of intersecting WZW defects each carrying the data of a non-boundary maximally symmetric WZW bi-brane, the world-sheet picture immediately suggests two alternative descriptions, namely: We can represent a point in the inter-bi-brane world-volume as a triple $\left(g, h_{\lambda}, h_{\mu}\right) \in \mathscr{G} \times \mathscr{C}_{\lambda} \times \mathscr{C}_{\mu}$ in which $g$ gives a reference value of the $\sigma$-model field, $h_{\lambda}$ is the jump at the first incoming defect line, $h_{\mu}$ is the jump at the second incoming defect line, and the latter two are constrained so that the total jump $h_{\lambda} \cdot h_{\mu}$ belongs to the second factor $\mathscr{C}_{\nu}$ of the world-volume of the outgoing bi-brane. The world-volume now arises as a disjoint union of (a subset of) all those $\mathscr{G} \times \mathscr{G}$-orbits within $\mathscr{G} \times \mathscr{C}_{\lambda} \times \mathscr{C}_{\mu}$ with respect to the action

$$
\begin{aligned}
(\mathscr{G} \times \mathscr{G}) \times\left(\mathscr{G} \times \mathscr{C}_{\lambda} \times \mathscr{C}_{\mu}\right) & \longrightarrow \mathscr{G} \times \mathscr{C}_{\lambda} \times \mathscr{C}_{\mu} \\
\left((x, y),\left(g, h_{\lambda}, h_{\mu}\right)\right) & \longmapsto\left(x \cdot g \cdot y^{-1}, \operatorname{Ad}_{y}\left(h_{\lambda}\right), \operatorname{Ad}_{y}\left(h_{\mu}\right)\right)
\end{aligned}
$$

that are mapped to $\mathscr{G} \times \mathscr{C}_{\nu}$ by id $\mathscr{G} \times \mathrm{m}$. This is the representation introduced and used in the original Refs. RS11a, Sus11. Equivalently, we may identify the world-volume of interest as a subspace in the fibred product

$$
Q_{\lambda \mathrm{m} \times \mathrm{pr}_{1}} Q_{\mu}=\left\{\left(\left(g, h_{\lambda}\right),\left(g^{\prime}, h_{\mu}\right)\right) \in Q_{\lambda} \times Q_{\mu} \quad \mid g \cdot h_{\lambda}=g^{\prime}\right\}
$$

of the world-volumes of the two 'incoming' bi-branes composed of (a subset of) all orbits of the $\mathscr{G} \times \mathscr{G}$-action

$$
\begin{aligned}
(\mathscr{G} \times \mathscr{G}) \times\left(Q_{\lambda \mathrm{m} \times \mathrm{pr}_{1}} Q_{\mu}\right) & \longrightarrow Q_{\lambda \mathrm{m} \times \mathrm{pr}_{1}} Q_{\mu} \\
\left((x, y),\left(\left(g, h_{\lambda}\right),\left(g \cdot h_{\lambda}, h_{\mu}\right)\right)\right) & \longmapsto\left(\left(x \cdot g \cdot y^{-1}, \operatorname{Ad}_{y}\left(h_{\lambda}\right)\right),\left(x \cdot g \cdot h_{\lambda} \cdot y^{-1}, \operatorname{Ad}_{y}\left(h_{\mu}\right)\right)\right)
\end{aligned}
$$

from the preimage of $Q_{\nu}$ under the map

$$
\begin{aligned}
\pi_{3}^{1,3}: Q_{\lambda \mathrm{m}_{\mathrm{pr}_{1}} Q_{\mu}} & \longrightarrow \mathscr{G} \times \mathscr{G} \\
\left(\left(g, h_{\lambda}\right),\left(g \cdot h_{\lambda}, h_{\mu}\right)\right) & \longmapsto\left(g, g^{-1} \cdot \mathrm{m}\left(g \cdot h_{\lambda}, h_{\mu}\right)\right) \equiv\left(g, h_{\lambda} \cdot h_{\mu}\right) .
\end{aligned}
$$

This is the representation that appears in Ref. RS11b. In general, there are many orbits of the type described in the fibred product $Q_{\lambda \mathrm{m}} \times_{\mathrm{pr}_{1}} Q_{\mu}$ of a given pair of $\mathcal{G}_{\mathrm{k}}$-bi-brane worldvolumes. We shall denote them as $\left[Q_{\lambda \mathrm{m} \times \mathrm{pr}_{1}} Q_{\mu}\right]_{(\nu, b)}$, adding an extra degeneracy label $b=0,1, \ldots$, to distinguish them from one another.

Definition 5.11. A maximally symmetric $\left(\mathcal{G}_{\mathrm{k}}, \mathcal{B}_{\mathrm{k}}\right)$-inter-bi-brane is a quintuple $\mathcal{J}_{\lambda, \mu}^{\nu}:=$ $\left(T_{\lambda, \mu}^{\nu}, \operatorname{pr}_{1}, \operatorname{pr}_{2}, \pi_{3}^{1,3}, \varphi_{\lambda, \mu}^{\nu}\right)$ with the following components:

(B.1) the world-volume

$$
T_{\lambda, \mu}^{\nu}:=\bigsqcup_{b=1}^{N_{\lambda, \mu}^{\nu}}\left[Q_{\lambda \mathrm{m} \times \mathrm{pr}_{1}} Q_{\mu}\right]_{(\nu, b)}
$$

given as a disjoint sum of those $\left(N_{\lambda, \mu}^{\nu}\right)$ orbits of the $\mathscr{G} \times \mathscr{G}$-action of Eq. (5.6) within the fibred product $Q_{\lambda \mathrm{m}^{\times} \mathrm{pr}_{1}} Q_{\mu}$ that are mapped to $Q_{\nu}$ by $\pi_{3}^{1,3}$ of Eq. (5.7) and that support a 2-isomorphism $\varphi_{\lambda, \mu}^{\nu}$ of (B.3);

(B.2) the canonical projections $\operatorname{pr}_{1}: T_{\lambda, \mu}^{\nu} \rightarrow Q_{\lambda}$ and $\mathrm{pr}_{2}: T_{\lambda, \mu}^{\nu} \rightarrow Q_{\mu}$, alongside map $\pi_{3}^{1,3}$ of Eq. (5.7); 
(B.3) a 2-isomorphism

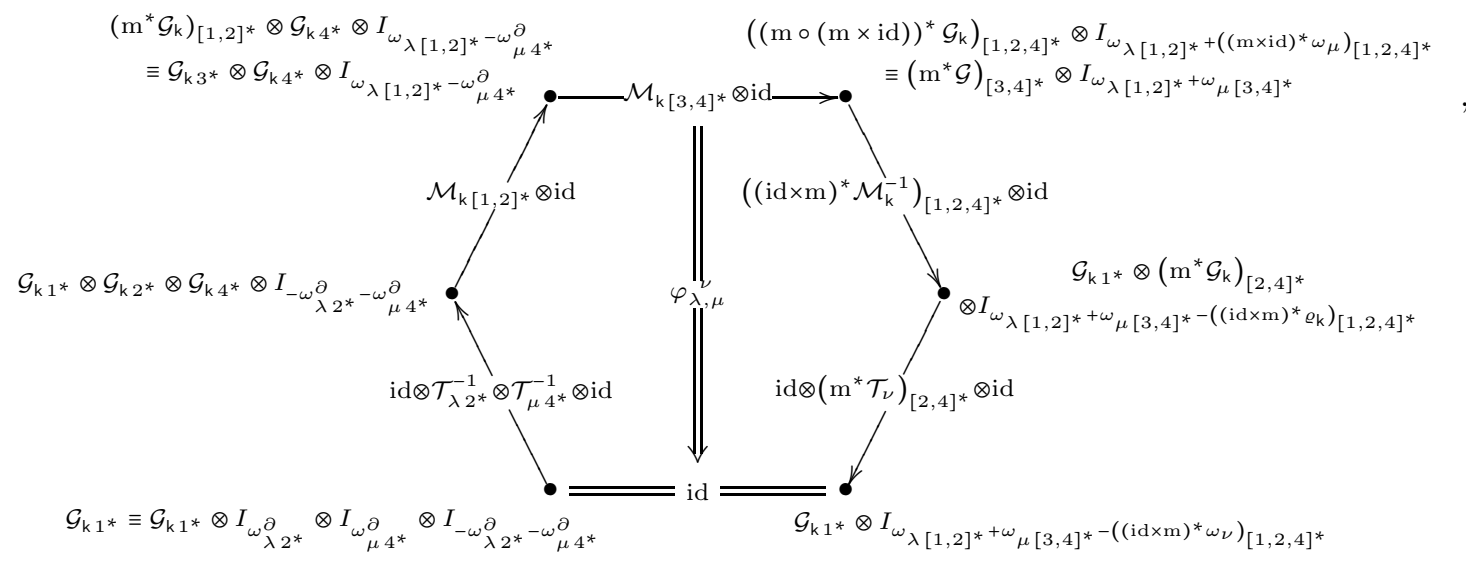

where the various pullback labels refer to the cartesian components in the decomposition $\mathscr{G} \times \mathscr{C}_{\lambda} \times \mathscr{G} \times \mathscr{C}_{\mu} \supset Q_{\lambda \mathrm{m} \times \mathrm{pr}_{1}} Q_{\mu}$.

Remark 5.12. The precise form of the $T_{\lambda, \mu}^{\nu}$ is known for $\mathscr{G}=\mathrm{SU}(2)$ exclusively, cf. Ref. RS11a]. It is conjectured in that paper, as well as in Ref. [RS11b], where partial evidence in favour of this conjecture is presented, that numbers $N_{\lambda, \mu}^{\nu}$ coincide for simply connected groups $\mathscr{G}$ with the structure constants of the Verlinde fusion ring (also known as the Verlinde dimensions) of the level-k WZW model.

5.5. Global gauge anomalies of the WZW-model amplitudes. The global gauge anomalies of the Feynman amplitudes of defect-free WZW models were analysed in Ref. GSW10 basing on Eq. (4.6) for pairs $(\chi, \varphi): \Sigma \rightarrow \mathrm{G} \times \mathscr{G}$ that generate the integral homology group $H_{2}(\mathrm{G} \times \mathscr{G})$. It was shown there that the relevant 2 -cycles that probe the global gauge anomaly come from $\Sigma=\mathbb{S}^{1} \times \mathbb{S}^{1}$ and

$$
(\chi, \varphi)\left(\mathrm{e}^{i \sigma_{1}}, \mathrm{e}^{i \sigma_{2}}\right)=\left(\exp \left[\sigma_{1} \tilde{p}^{\vee}\right], \exp \left[\sigma_{2} p^{\vee}\right]\right)
$$

with $\tilde{p}^{\vee}, p^{\vee} \in \mathfrak{t}$ such that $\mathrm{e}^{2 \pi \tilde{p}^{\vee}} \in \tilde{Z}$ and $e^{2 \pi p^{\vee}} \in Z$. For such fields $\chi \cdot \varphi=\varphi$ resulting in the relation

$$
\operatorname{Hol}_{\mathcal{D}}((\chi, \varphi))=\exp \left[2 \pi \mathrm{iktr} \operatorname{tr}_{\mathfrak{g}} \tilde{p}^{\vee} p^{\vee}\right] .
$$

The triviality of the right-hand side gives an easily verifiable condition. It was used in Ref. GSW10 to detect the presence of global anomalies in multiple cases of defect-free WZW $\sigma$-models with non-simply connected target groups $\mathscr{G}$. This was further studied in Ref. dif].

Now add a circular defect $\Gamma \subset \Sigma$ with the field $\left.\varphi\right|_{\Gamma}$ mapping into the world-volume $Q_{\lambda}$ of a non-boundary maximally symmetric $\mathcal{G}_{\mathrm{k}}$ bi-brane. If the bulk is without global gauge anomaly, then the source of remaining global anomaly is the holonomy of the flat bundle $D$, defined in Eq. (4.11), around the 1-cycle $\left.(\chi, \varphi)\right|_{\Gamma}$ in $\mathrm{G} \times Q_{\lambda}$, cf. Eq. 4.12). Relation (4.13) now reduces to the identity

$$
\left.\operatorname{Hol}_{D}\left(\left.(\chi, \varphi)\right|_{\Gamma}\right)\right)=\operatorname{Hol}_{\mathcal{G}_{k}, \Phi_{\lambda}}(\chi \cdot \varphi \mid \Gamma) \operatorname{Hol}_{\mathcal{G}_{k}, \Phi_{\lambda}}(\varphi \mid \Gamma)^{-1} \mathrm{e}^{-i \int_{\Sigma \backslash \Gamma} \phi^{*} \rho_{\chi^{*} \theta_{L}}}
$$

since the 1 -form $\lambda_{\mathrm{A}}=0$ in the case of maximally symmetric $\mathcal{G}_{\mathrm{k}}$-bi-branes. To test the contribution of circular defects to the global gauge anomaly, one should take $\chi$ and $(\varphi \mid \Gamma)$ such that $\left.(\chi, \varphi)\right|_{\Gamma}$ generate the kernel of the map

$$
\left(\iota_{2}^{(1)}\right)_{*}-\left(\iota_{1}^{(1)}\right)_{*}: H_{1}\left(\mathrm{G} \times Q_{\lambda}\right) \rightarrow H_{1}(\mathrm{G} \times \mathscr{G}),
$$

cf. Appendix B. One has

$$
H_{1}\left(\mathrm{G} \times Q_{\lambda}\right) \cong H_{1}(\mathrm{G}) \oplus H_{1}(\mathscr{G}) \oplus H_{1}\left(\mathscr{C}_{\lambda}\right),
$$

and it is easy to see that the kernel of $(5.10)$ corresponds to the subgroup $H_{1}(\mathrm{G}) \oplus H_{1}(\mathscr{G}) \subset$ $H_{1}\left(\mathrm{G} \times Q_{\lambda}\right)$. It is then enough to take $\Sigma=\mathbb{S}^{1} \times \mathbb{S}^{1}, \Gamma=\{1\} \times \mathbb{S}^{1}$ and

$$
\chi\left(e^{i \sigma_{1}}, e^{i \sigma_{2}}\right)=\exp \left[\sigma_{2} \tilde{p}^{\vee}\right],
$$




$$
\begin{aligned}
\varphi\left(e^{i \sigma_{1}}, e^{i \sigma_{2}}\right) & =\exp \left[\sigma_{1} \lambda+\sigma_{2} p^{\vee}\right] \text { for } \sigma_{1} \neq 0 \bmod 2 \pi, \\
\varphi\left(1, e^{i \sigma_{2}}\right) & =\left(\exp \left[\sigma_{2} p^{\vee}\right], \exp [2 \pi \lambda]\right) \in Q_{\lambda}
\end{aligned}
$$

with $\tilde{p}^{\vee}$ and $p^{\vee}$ as before. In this case, $\chi \cdot \varphi|\Gamma=\varphi| \Gamma$ and identity (5.9) gives

$$
\operatorname{Hol}_{D}\left(\left.(\chi, \varphi)\right|_{\Gamma}\right)=\exp \left[2 \pi i k \operatorname{tr}_{\mathfrak{g}} \lambda \tilde{p}^{\vee}\right]
$$

Hence, the absence of the global gauge anomaly requires that $k \operatorname{tr}_{\mathfrak{g}} \lambda \tilde{p}^{\vee} \in \mathbb{Z}$ for all $\tilde{p}^{\vee} \in \mathfrak{t}$ such that $\mathrm{e}^{2 \pi \tilde{p}^{\vee}} \in \tilde{Z}$, i.e. for $\tilde{p}^{\vee}$ in the coweight lattice. This holds iff $\mathrm{k} \lambda$ is in the root lattice of $\mathfrak{g}$. For the other non-boundary maximally symmetric $\mathcal{G}_{\mathrm{k}}$-bi-branes, the global gauge anomaly occurs. Note that the right-hand side of Eq. (5.12) does not depend on the winding of $\varphi$ along the second $\mathbb{S}^{1}$ factor in $\Sigma$. This will find its explanation in the cohomological analysis of Section 11, cf. Corollary 11.16, where it will be shown that the isomorphism class of the flat line bundle $D$ (given by its holonomy) is represented by a cohomology class in $H^{1}(\mathrm{G}, \mathrm{U}(1)) \subset H^{1}\left(\mathrm{G} \times Q_{\lambda}, \mathrm{U}(1)\right)$.

For a single boundary maximally-symmetric $\mathcal{G}_{\mathrm{k}}$-bi-brane with world-volume $\mathscr{C}_{\lambda}$, a similar calculation gives

$$
\operatorname{Hol}_{D}\left(\left.(\chi, \varphi)\right|_{\Gamma}\right)=1
$$

since the kernel of the map

$$
\left(\iota_{2}^{(1)}\right)_{*}-\left(\iota_{1}^{(1)}\right)_{*}: H_{1}\left(\mathrm{G} \times \mathscr{C}_{\lambda}\right) \rightarrow H_{1}(\mathrm{G} \times(\mathscr{G} \sqcup\{\bullet\})),
$$

to which the homology class generated by $\left.(\chi, \varphi)\right|_{\Gamma}$ belongs, is easily seen to vanish. This is no more the case for general maximally symmetric $\mathcal{G}_{\mathrm{k}}$-branes. Taking, for example, the disjoint union of two $\mathcal{G}_{\mathrm{k}}$-branes with world-volume $\mathscr{C}_{\lambda_{1}} \sqcup \mathscr{C}_{\lambda_{2}}$, one sees that the kernel of the map

$$
\left(\iota_{2}^{(1)}\right)_{*}-\left(\iota_{1}^{(1)}\right)_{*}: H_{1}\left(\mathrm{G} \times\left(\mathscr{C}_{\lambda_{1}} \sqcup \mathscr{C}_{\lambda_{2}}\right)\right) \rightarrow H_{1}(\mathrm{G} \times(\mathscr{G} \sqcup\{\bullet\}))
$$

is nontrivial. The 1-cycles that probe the global anomaly in that case correspond to $\Sigma=$ $\mathbb{S}^{1} \times \mathbb{S}^{1}, \Gamma=\{1\} \times \mathbb{S}^{1} \sqcup\{-1\} \times \mathbb{S}^{1}$, and

$$
\begin{aligned}
\chi\left(e^{i \sigma_{1}}, e^{i \sigma_{2}}\right) & =\exp \left[\sigma_{2} \tilde{p}^{\vee}\right], \\
\varphi\left(e^{i \sigma_{1}}, e^{i \sigma_{2}}\right) & =\exp \left[2 \pi \lambda_{1}+2 \sigma_{1}\left(\lambda_{2}-\lambda_{1}\right)\right] \text { for } 0<\sigma_{1}<\pi \bmod 2 \pi, \\
\varphi\left(e^{i \sigma_{1}}, e^{i \sigma_{2}}\right) & =\bullet \text { for } \pi<\sigma_{1}<2 \pi \bmod 2 \pi, \\
\varphi\left(1, e^{i \sigma_{2}}\right) & =\exp \left[2 \pi \lambda_{1}\right] \in \mathscr{C}_{\lambda_{1}} \\
\varphi\left(-1, e^{i \sigma_{2}}\right) & =\exp \left[2 \pi \lambda_{2}\right] \in \mathscr{C}_{\lambda_{2}}
\end{aligned}
$$

for which

$$
\operatorname{Hol}_{\mathcal{D}}((\chi, \varphi))=\exp \left[2 \pi i k \operatorname{tr}_{\mathfrak{g}}\left(\lambda_{1}-\lambda_{2}\right) \tilde{p}^{\vee}\right] .
$$

Hence, the absence of contributions to the global anomaly from circular defects requires, in this case, that $k\left(\lambda_{1}-\lambda_{2}\right)$ belongs to the root lattice. As will follow from the analysis of Section 11, and in particular from Corollary 11.16, this is also the necessary condition for the absence of such contributions. Similar condition holding for all pair differences $\mathrm{k}\left(\lambda_{i}-\lambda_{j}\right)$ is required by the absence of boundary contributions to the global gauge anomaly for general maximally symmetric $\mathcal{G}_{k}$-branes with world-volumes that are disjoint unions of more than two conjugacy classes.

The analysis in Section 11 below, and in particular Corollary 11.27, shows also that the $\mathrm{U}(1)$-valued functions $d_{n}$ that appear in the identity (4.20) are trivial in the case in question so that introduction of maximally symmetric inter-bi-branes and intersecting defects does not lead to further global gauge anomalies. 


\section{A GRoupoidAl INTERPREtation OF THE CONSTRAints}

In this section, we reappraise the constraints for a consistent gauging, Eqs. (3.6)-(3.8), in more geometric terms, and - in so doing - perform a more precise identification of the structure underlying the rigid symmetries of the $\sigma$-model. This goal will be achieved by establishing a straightforward link between the $\left(\mathrm{H}, \omega ; \Delta_{Q}\right)$-twisted bracket structure $\mathfrak{M}^{\left(\mathrm{H}, \omega ; \Delta_{Q}\right)} \mathscr{F}$ on the distinguished $\sigma$-symmetric sections of the total generalised tangent bundle $\mathrm{E} \mathscr{F}$, introduced in Section 2.3, and the (action-)groupoid structure. The relevance of the latter transpires from our discussion of large gauge transformations in Section 4 and will be demonstrated convincingly in Section 10, where we extend the gauging procedure to nontrivial gauge bundles.

In what follows, we assume knowledge of basic notions of the theory of Lie groupoids and Lie algebroids. For the reader's convenience, we recall the relevant facts in Appendix C.

Let us, first, be more specific about the algebraic structure on the $\sigma$-symmetric sections of $\mathrm{E} \mathscr{F}$. We have

Proposition 6.1. Sus12, Prop. 5.10] Let $\Gamma_{\sigma}(\mathrm{E} \mathscr{F})$ be the subspace of the $\sigma$-symmetric sections, defined in Proposition 2.2X, of the total generalised tangent bundle $\mathrm{E} \mathscr{F}$, introduced in Definition 2.25, over the target space $\mathscr{F}=M \sqcup Q \sqcup T$ of the string background $\mathfrak{B}$, as characterised in Definition 2.1. Furthermore, let $\mathfrak{M}_{\sigma}^{\left(\mathrm{H}, \omega ; \Delta_{Q}\right)} \mathscr{F}$ be the restriction of the $\left(\mathrm{H}, \omega ; \Delta_{Q}\right)$ twisted bracket structure $\mathfrak{M}^{\left(\mathrm{H}, \omega ; \Delta_{Q}\right)} \mathscr{F}$ on smooth sections of $\mathrm{E} \mathscr{F}$ to $\Gamma_{\sigma}(\mathrm{E} \mathscr{F})$. The subspace $\alpha_{\mathrm{T} \mathscr{F}}\left(\Gamma_{\sigma}(\mathrm{E} \mathscr{F})\right)$ is a Lie subalgebra in the Lie algebra of vector fields on $\mathscr{F}$ and it is isomorphic with the Lie algebra $\mathfrak{g}$ of the Lie group $\mathrm{G}$ of the rigid $\sigma$-model symmetries. Fix a basis $\left\{\mathscr{F} \mathscr{K}_{a}\right\}$ in $\alpha_{\mathrm{T} \mathscr{F}}\left(\Gamma_{\sigma}(\mathrm{E} \mathscr{F})\right)$ composed of the fundamental vector fields on $\mathscr{F}$ corresponding to the generators $t_{a}$ of $\mathfrak{g}$. The associated $\sigma$-symmetric sections $\mathfrak{K}_{a}$ of $\Gamma_{\sigma}(\mathrm{E} \mathscr{F})$, with restrictions

$$
\left.\mathfrak{K}_{a}\right|_{M}={ }^{M} \mathscr{K}_{a} \oplus \kappa_{a},\left.\quad \mathfrak{K}_{a}\right|_{Q}={ }^{Q} \mathscr{K}_{a} \oplus k_{a},\left.\quad \mathfrak{K}_{a}\right|_{T}={ }^{T_{n}} \mathscr{K}_{a}
$$

in which the smooth 1-forms $\kappa_{a}$ on $M$ and the smooth functions $k_{a}$ on $Q$ are related as in Eqs. (2.27)-(2.29), satisfy the algebra

$$
\llbracket \mathfrak{K}_{a}, \mathfrak{K}_{b} \rrbracket^{\left(\mathrm{H}, \omega ; \Delta_{Q}\right)}=f_{a b c} \mathfrak{K}_{c}+0 \oplus \alpha_{a b}
$$

with

$$
\alpha_{a b} \mid \mathscr{M}= \begin{cases}\mathscr{L}_{a} \kappa_{b}-f_{a b c} \kappa_{c}-\mathrm{d} c_{(a b)} & \text { on } \quad \mathscr{M}=M \\ \mathscr{L}_{a} k_{b}-f_{a b c} k_{c}+\Delta_{Q} c_{(a b)} & \text { on } \left.\quad \mathscr{M}=Q \quad, \quad c_{(a b)}=\left(\mathfrak{K}_{a}, \mathfrak{K}_{b}\right)\right\lrcorner . \\ 0 & \text { on } \quad \mathscr{M}=T_{n}\end{cases}
$$

It is well-known, cf. Ref. Gua03, that a non-vanishing scalar product $(\cdot, \cdot)\lrcorner$ of sections of the Courant algebroid on $\mathrm{E}^{(1,1)} M$ violates the Leibniz identity and the Jacobi identity for the (Courant) bracket, and so prevents the Courant algebroid from becoming a Lie algebroid. A similar situation occurs in the present setup. In particular, we find

$$
\left.\llbracket \mathfrak{V}, f \mathfrak{W} \rrbracket^{\left(\mathrm{H}, \omega ; \Delta_{Q}\right)}=f \llbracket \mathfrak{V}, \mathfrak{W} \rrbracket^{\left(\mathrm{H}, \omega ; \Delta_{Q}\right)}+\left(\imath_{\alpha_{\top} \mathscr{F}}(\mathfrak{V}) \mathrm{d} f\right) \mathfrak{W}-0 \oplus \frac{1}{2}(\mathfrak{V}, \mathfrak{W})\right\lrcorner \mathrm{d} f(6.2)
$$

for arbitrary $\mathfrak{V}, \mathfrak{W} \in \Gamma(\mathrm{E} \mathscr{F})$ and $f \in C^{\infty}(\mathscr{F}, \mathbb{R})$, so that, once again, the Leibniz identity is obstructed by a non-vanishing scalar product. Inspection of the previous proposition yields

Proposition 6.2. Let $\left\{\mathfrak{K}_{a}\right\}$ be the basis, introduced in Proposition 6.1 , of the subspace of $\sigma$-symmetric sections of the total generalised tangent bundle EF over the target space $\mathscr{F}=M \sqcup Q \sqcup T$ of the string background $\mathfrak{B}$ of Definition 2.1. The triple

$$
\mathfrak{S}_{\mathfrak{B}}:=\left(\oplus_{a=1}^{\operatorname{dim} \mathfrak{g}} C^{\infty}(\mathscr{F}, \mathbb{R}) \mathfrak{K}_{a}, \llbracket \cdot, \cdot \rrbracket^{\left(\mathrm{H}, \omega ; \Delta_{Q}\right)}, \alpha_{\mathrm{T} \mathscr{F}}\right)
$$

carries a canonical structure of a Lie algebroid iff the $\mathfrak{K}_{a}$ satisfy constraints corresponding to Eqs. (3.6)- 3.8).

Proof. The structure of a Lie algebroid on $\mathfrak{S}_{\mathfrak{B}}$ means that the Leibniz and Jacobi identities for $\llbracket \cdot, \cdot \rrbracket^{\left(\mathrm{H}, \omega ; \Delta_{Q}\right)}$ hold true on the subspace $\oplus_{a=1}^{\operatorname{dim} \mathfrak{g}} C^{\infty}(\mathscr{F}, \mathbb{R}) \mathfrak{K}_{a}$, the latter being closed 
under the bracket. Inspection of Eq. (6.2) immediately shows that the Leibniz identity is tantamount to the isotropy of the subspace,

$$
\mathrm{c}_{(a b)}=0,
$$

that is to condition (3.8). The closure condition then takes the form

$$
\alpha_{a b}=0,
$$

which, upon taking into account the earlier Eq. (6.3), becomes the conjunction of conditions (3.6) and (3.7). The Jacobi identity for the bracket $\llbracket \cdot, \cdot \rrbracket^{\left(\mathrm{H}, \omega ; \Delta_{Q}\right)}$ restricted to the thus constrained subspace is simply the Jacobi identity for the structure constants of $\mathfrak{g}$.

The above considerations lead us to

Definition 6.3. The Lie algebroid $\mathfrak{S}_{\mathfrak{B}}$ introduced in Proposition 6.2 will be called the gauge-symmetry $(\mathrm{Lie})$ algebroid of string background $\mathfrak{B}$. Similarly, the structure

$$
\mathrm{S}_{\mathfrak{B}}:=\left(\mathrm{G} \ltimes \mathscr{F},\left(\iota_{\alpha} \mid \alpha=1,2\right),\left(\pi_{n}^{k, k+1} \mid k=1,2, \ldots, n, n \geq 3\right)\right),
$$

consisting of the action groupoid $\mathrm{G} \ltimes \mathscr{F}$ and of the G-maps of Proposition 2.21, will be termed the gauge-symmetry (Lie) groupoid of string background $\mathfrak{B}$.

We conclude the present section with a theorem that relates the two constructs, $\mathfrak{S}_{\mathfrak{B}}$ and $\mathbf{S}_{\mathfrak{B}}$, and thus provides us with a novel interpretation of the constraints for a consistent gauging.

Theorem 6.4. Let $\mathfrak{B}$ be a string background from Definition 2.1, and let $\mathfrak{g} \times \mathscr{F}$ be the tangent algebroid, as characterised in Definition C.?, of the gauge-symmetry groupoid $\mathrm{S}_{\mathfrak{B}}$ of Definition 6.5. Finally, let $\mathfrak{s}_{\mathfrak{B}}$ be the gauge-symmetry algebroid of background $\mathfrak{B}$ introduced in the same definition. Then, there exists a canonical isomorphism

$$
\mathfrak{s}_{\mathfrak{B}} \cong \mathfrak{g} \ltimes \mathscr{F}
$$

in the sense of Definition C.1 of Appendix Q

A constructive proof of the theorem is given in Appendix D.

\section{The gauging vs. The $\mathfrak{g}$-EQuivariance of StRing BaCKGRounds}

In the previous section, we established a purely geometric interpretation of the (infinitesimal) conditions for a consistent gauging, without any direct reference to the differential-geometric structures present over the target space Here, we shall change the angle once more and reinterpret the same conditions in terms of a $\mathfrak{g}$-equivariant extension of the Deligne hypercohomology that captures the local description of the target-space structure $\left(\mathcal{G}, \Phi, \varphi_{n} \mid n \geq 3\right)$. This is to be viewed as a natural step towards the full-fledged G-equivariant structure on $\mathfrak{B}$ that will be developed in order to analyse topologically nontrivial sectors of gauged $\sigma$-model.

7.1. The local description of the background structure. Below, we briefly review the sheaf-cohomological description of the target-space gerbe $\mathcal{G}$, the bi-brane 1-isomorphism $\Phi$ and the inter-bi-brane 2-isomorphisms $\varphi_{n}$. We confine ourselves to the basic elements of the description, relegating the full-blown discussion to Section 11.1 in which we employ the cohomological description in a classification of G-equivariant structures on string backgrounds.

The point of departure is the Deligne complex

$$
\mathcal{D}(4)^{\bullet}: 0 \rightarrow \underline{2 \pi \mathbb{Z}}_{\mathscr{F}} \rightarrow \underline{\Omega}^{0}(\mathscr{F}) \stackrel{\mathrm{d}}{\rightarrow} \underline{\Omega}^{1}(\mathscr{F}) \stackrel{\mathrm{d}}{\rightarrow} \underline{\Omega}^{2}(\mathscr{F}) \stackrel{\mathrm{d}}{\rightarrow} \underline{\Omega}^{3}(\mathscr{F})
$$

of the following differential sheaves over the target space: the sheaf $\underline{2 \pi \mathbb{Z}}_{\mathscr{F}}$ of locally constant $2 \pi \mathbb{Z}$-valued functions on $\mathscr{F}$ (trivially injected in its successor), and the sheaves $\underline{\Omega}^{p}(\mathscr{F}), p=$ $0,1,2$ of locally smooth (real) $p$-forms on $\mathscr{F}$. Given a choice $\mathcal{O}^{\mathscr{F}}=\left\{O_{i}^{\mathscr{F}}\right\}_{i \in \mathscr{I} \mathscr{F}}$ of a good open cover of $\mathscr{F}$ (i.e. an open cover with contractible non-empty multiple intersections of its

\footnotetext{
${ }^{4}$ In fact, there is a natural relation between the $\left(\mathrm{H}, \omega ; \Delta_{Q}\right)$-twisted bracket structure $\mathfrak{M}^{\left(\mathrm{H}, \omega ; \Delta_{Q}\right) \mathscr{F}}$ on $\sigma$-symmetric sections of the total generalised tangent bundle $\mathrm{E} \mathscr{F}$ and the 2-category of bundle gerbes with curving and connection over $\mathscr{F}$. Over $M$, it boils down to the Hitchin morphism, first reported in Ref. Hit06, between the H-twisted Courant algebroid on $\mathrm{E}^{(1,1)} M$ and an untwisted Courant algebroid on the generalised tangent bundle over $M$ twisted by (local data of) the gerbe $\mathcal{G}$ of curvature $\mathrm{H}$. The relation is worked out in all generality in Ref. [Sus12]. We shall not pursue this aspect of $\sigma$-model symmetries in the present paper.
} 
elements, to be denoted as $\mathcal{O}_{i j}^{\mathscr{F}}=\mathcal{O}_{i}^{\mathscr{F}} \cap \mathcal{O}_{j}^{\mathscr{F}}, \mathcal{O}_{i j k}^{\mathscr{F}}=\mathcal{O}_{i}^{\mathscr{F}} \cap \mathcal{O}_{j}^{\mathscr{F}} \cap \mathcal{O}_{k}^{\mathscr{F}}$ etc.), the Deligne complex is extended in the direction of the associated Cech cohomology, whereby the standard CechDeligne double complex $\check{C}^{\bullet}\left(\mathcal{O}^{\mathscr{F}}, \mathcal{D}(4)^{\bullet}\right)$ is formed. The cohomology that describes local data of $\mathcal{G}, \Phi$ and $\varphi_{n}$, as well as the usual (gauge) ambiguities in their definition is exactly the cohomology of that double complex, defined as the cohomology of the diagonal subcomplex

$$
A_{\mathscr{F}}^{\bullet}\left(\mathcal{O}^{\mathscr{F}}\right)=\bigoplus_{r=0,1, \ldots} A_{\mathscr{F}}^{r}\left(\mathcal{O}^{\mathscr{F}}\right), \quad A_{\mathscr{F}}^{r}\left(\mathcal{O}^{\mathscr{F}}\right)=\bigoplus_{p+q=r} \check{C}^{p}\left(\mathcal{O}^{\mathscr{F}}, \mathcal{D}(4)^{q}\right)
$$

with respect to the Deligne differential $D_{r}: A_{\mathscr{F}}^{r}\left(\mathcal{O}^{\mathscr{F}}\right) \rightarrow A_{\mathscr{F}}^{r+1}\left(\mathcal{O}^{\mathscr{F}}\right)$. We give explicit formulæ for the latter on representative Cech-Deligne cochains for the first few values of $r$. Thus, for cochains

$$
\begin{aligned}
\left(p_{i}\right) \in A_{\mathscr{F}}^{0}\left(\mathcal{O}^{\mathscr{F}}\right) & =\check{C}^{0}\left(\mathcal{O}^{\mathscr{F}}, \underline{\underline{Z}}_{\mathscr{F}}\right), \\
\left(f_{i}, q_{i j}\right) \in A_{\mathscr{F}}^{1}\left(\mathcal{O}^{\mathscr{F}}\right)= & \check{C}^{0}\left(\mathcal{O}^{\mathscr{F}}, \underline{\Omega}^{0}(\mathscr{F})\right) \oplus \check{C}^{1}\left(\mathcal{O}^{\mathscr{F}}, \underline{2 \pi \mathbb{Z}} \mathscr{F}\right), \\
\left(P_{i}, k_{i j}, r_{i j k}\right) \in A_{\mathscr{F}}^{2}\left(\mathcal{O}^{\mathscr{F}}\right)= & \check{C}^{0}\left(\mathcal{O}^{\mathscr{F}}, \underline{\Omega}^{1}(\mathscr{F})\right) \oplus \check{C}^{1}\left(\mathcal{O}^{\mathscr{F}}, \underline{\Omega}^{0}(\mathscr{F})\right) \oplus \check{C}^{2}\left(\mathcal{O}^{\mathscr{F}}, \underline{2 \pi \mathbb{Z}} \mathscr{F}\right), \\
\left(B_{i}, A_{i j}, h_{i j k}, s_{i j k l}\right) \in A_{\mathscr{F}}^{3}\left(\mathcal{O}^{\mathscr{F}}\right)= & \check{C}^{0}\left(\mathcal{O}^{\mathscr{F}}, \underline{\Omega}^{2}(\mathscr{F})\right) \oplus \check{C}^{1}\left(\mathcal{O}^{\mathscr{F}}, \underline{\Omega}^{1}(\mathscr{F})\right) \oplus \check{C}^{2}\left(\mathcal{O}^{\mathscr{F}}, \underline{\Omega}^{0}(\mathscr{F})\right) \\
& \oplus \check{C}^{3}\left(\mathcal{O}^{\mathscr{F}}, \underline{2 \pi \mathbb{Z}} \mathscr{F}\right),
\end{aligned}
$$

we have

$$
\begin{aligned}
D_{0}\left(p_{i}\right)= & \left(p_{i},\left.\left(p_{j}-p_{i}\right)\right|_{\mathcal{O}_{i j}^{\mathscr{F}}}\right), \\
D_{1}\left(f_{i}, q_{i j}\right)= & \left(\mathrm{d} f_{i},\left.\left(f_{i}-f_{j}\right)\right|_{\mathcal{O}_{i j}^{\mathscr{F}}}+q_{i j},\left.\left(q_{j k}-q_{i k}+q_{i j}\right)\right|_{\mathcal{O}_{i j k}^{\mathscr{F}}}\right), \\
D_{2}\left(P_{i}, k_{i j}, r_{i j k}\right)= & \left(\mathrm{d} P_{i},\left.\left(P_{j}-P_{i}\right)\right|_{\mathcal{O}_{i j}^{\mathscr{F}}}+\mathrm{d} k_{i j},\left.\left(-k_{j k}+k_{i k}-k_{i j}\right)\right|_{\mathcal{O}_{i j k}^{\mathscr{F}}}+r_{i j k},\right. \\
& \left.\left.\left(r_{j k l}-r_{i k l}+r_{i j l}-r_{i j k}\right)\right|_{\mathcal{O}_{i j k l}^{\mathscr{F}}}\right), \\
D_{3}\left(B_{i}, A_{i j}, h_{i j k}, s_{i j k l}\right)= & \left(\mathrm{d} B_{i},\left.\left(B_{j}-B_{i}\right)\right|_{\mathcal{O}_{i j}^{\mathscr{F}}}-\mathrm{d} A_{i j},\left.\left(A_{j k}-A_{i k}+A_{i j}\right)\right|_{\mathcal{O}_{i j k}^{\mathscr{F}}}+\mathrm{d} h_{i j k},\right. \\
& \left.\left(-h_{j k l}+h_{i k l}-h_{i j l}+h_{i j k}\right)\right|_{\mathcal{O}_{i j k l}^{\mathscr{F}}}+s_{i j k l}, \\
& \left.\left.\left(s_{j k l m}-s_{i k l m}+s_{i j l m}-s_{i j k m}+s_{i j k l}\right)\right|_{\mathcal{O}_{i j k l m}^{\mathscr{F}}}\right) .
\end{aligned}
$$

We may now describe the gerbe $\mathcal{G}$ with the curvature 3 -form $\mathrm{H}$ in terms of its local data $\left(B_{i}, A_{i j}, h_{i j k}, s_{i j k l}\right) \in A_{M}^{3}\left(\mathcal{O}^{M}\right)$ satisfying the relation

$$
D_{3}\left(B_{i}, A_{i j}, h_{i j k}, s_{i j k l}\right)=\left(\left.\mathrm{H}\right|_{\mathcal{O}_{i}^{M}}, 0,0,0,0\right) .
$$

Similarly, the 1-isomorphism $\Phi$ may be described by local data $\left(P_{i}, k_{i j}, r_{i j k}\right) \in A_{Q}^{2}\left(\mathcal{O}^{Q}\right)$ verifying the identity

$$
\begin{aligned}
& \iota_{1}^{*}\left(B_{\phi_{1}(i)}, A_{\phi_{1}(i) \phi_{1}(j)}, h_{\phi_{1}(i) \phi_{1}(j) \phi_{1}(k)}, s_{\phi_{1}(i) \phi_{1}(j) \phi_{1}(k) \phi_{1}(l)}\right)+D_{2}\left(P_{i}, k_{i j}, r_{i j k}\right) \\
& =\iota_{2}^{*}\left(B_{\phi_{2}(i)}, A_{\phi_{2}(i) \phi_{2}(j)}, h_{\phi_{2}(i) \phi_{2}(j) \phi_{2}(k)}, s_{\phi_{2}(i) \phi_{2}(j) \phi_{2}(k) \phi_{2}(l)}\right)+\left(\left.\omega\right|_{\mathcal{O}_{i}^{Q}}, 0,0,0\right),
\end{aligned}
$$

written in terms of the index maps $\phi_{\alpha}: \mathscr{I}^{Q} \rightarrow \mathscr{I}^{M}$ that cover (or Čech-extend) the $\iota_{\alpha}$ for covers of $M$ and $Q$ chosen in conformity with the condition

$$
\iota_{\alpha}\left(\mathcal{O}_{i}^{Q}\right) \subset \mathcal{O}_{\phi_{\alpha}(i)}^{M},
$$

cf. Ref. RS09. Finally, local data $\left(f_{n i}, q_{n i j}\right) \in A_{T_{n}}^{2}\left(\mathcal{O}^{T_{n}}\right)$ of the 2-isomorphism $\varphi_{n}$ are readily seen to obey

$$
\begin{array}{r}
\sum_{k=1}^{n} \varepsilon_{n}^{k, k+1} \pi_{n}^{k, k+1 *}\left(P_{\psi_{n}^{k, k+1}(i)}, k_{\psi_{n}^{k, k+1}(i) \psi_{n}^{k, k+1}(j)}, r_{\psi_{n}^{k, k+1}(i) \psi_{n}^{k, k+1}(j) \psi_{n}^{k, k+1}(k)}\right) \\
+D_{1}\left(f_{n i}, q_{n i j}\right)=0,
\end{array}
$$


where the index maps $\psi_{n}^{k, k+1}: \mathscr{I}^{T_{n}} \rightarrow \mathscr{I}^{Q}$ covering the inter-bi-brane maps $\pi_{n}^{k, k+1}$ have been used for covers of $Q$ and $T_{n}$ chosen such that the relations

$$
\pi_{n}^{k, k+1}\left(\mathcal{O}_{i}^{T_{n}}\right) \subset \mathcal{O}_{\psi_{n}^{k, k+1}(i)}^{Q}
$$

hold true.

Convention 7.1. Following Ref. RS09, we shall use the symbols $\check{\iota}_{\alpha}$ and $\check{\pi}_{n}^{k, k+1}$ for the Čech-extended maps, e.g., given $b:=\left(B_{i}, A_{i j}, h_{i j k}, s_{i j k l}\right) \in A_{M}^{3}\left(\mathcal{O}^{M}\right)$ and $p=\left(P_{i}, k_{i j}, r_{i j k}\right) \epsilon$ $A_{Q}^{2}\left(\mathcal{O}^{Q}\right)$, we shall write

$$
\begin{aligned}
\check{\iota}_{\alpha}^{*} b & \equiv \iota_{\alpha}^{*}\left(B_{\phi_{\alpha}(i)}, A_{\phi_{\alpha}(i) \phi_{\alpha}(j)}, h_{\phi_{\alpha}(i) \phi_{\alpha}(j) \phi_{\alpha}(k)}, s_{\phi_{\alpha}(i) \phi_{\alpha}(j) \phi_{\alpha}(k) \phi_{\alpha}(l)}\right), \\
\check{\pi}_{n}^{k, k+1 *} p & \equiv \pi_{n}^{k, k+1 *}\left(P_{\psi_{n}^{k, k+1}(i)}, k_{\psi_{n}^{k, k+1}(i) \psi_{n}^{k, k+1}(j)}, r_{\psi_{n}^{k, k+1}(i) \psi_{n}^{k, k+1}(j) \psi_{n}^{k, k+1}(k)}\right) .
\end{aligned}
$$

7.2. g-equivariant structures. We shall now gradually descend in the diagonal subcomplex of the triple complex obtained by extending $\check{C}^{\bullet}\left(\mathcal{O}^{\mathscr{F}}, \mathcal{D}(4)^{\bullet}\right)$ in the direction of $\mathfrak{g}$-cohomology, as dictated by the constraints for a consistent gauging derived earlier. Thus, we find

Proposition 7.2. Let $M$ be a $\mathrm{G}$-space with a gerbe $\mathcal{G}$ of a $\mathrm{G}$-invariant curvature $\mathrm{H}$ over $i t$, and let $b:=\left(B_{i}, A_{i j}, h_{i j k}, s_{i j k l}\right) \in A_{M}^{3}\left(\mathcal{O}^{M}\right)$ be local data of $\mathcal{G}$ with respect to a good open cover $\mathcal{O}^{M}=\left\{\mathcal{O}_{i}^{M}\right\}_{i \in \mathscr{I} M}$ of $M$, so that, in particular,

$$
D_{3} b=\left(\left.\mathrm{H}\right|_{\mathcal{O}_{i}^{M}}, 0,0,0,0\right) .
$$

Given $\kappa_{a} \in \Omega^{1}(M), a=1,2, \ldots, \operatorname{dim} \mathfrak{g}$, that satisfy Eq. (2.27), write

$$
\Upsilon_{a}:=\left(-\left.\kappa_{a}\right|_{\mathcal{O}_{i}^{M}}+\imath_{a} B_{i}, \imath_{a} A_{i j}, 0\right) \in A_{M}^{2}\left(\mathcal{O}^{M}\right)
$$

and

$$
\gamma_{a b}:=\left(\left.c_{b a}\right|_{\mathcal{O}_{i}^{M}}+\imath_{a} \imath_{b} B_{i}, 0\right) \in A_{M}^{1}\left(\mathcal{O}^{M}\right)
$$

for ${ }^{M} \mathscr{K}_{a}$ the fundamental vector fields on $M$ from Definition 2.10. Relations (3.6) and (3.8) are then satisfied iff there exists a $\mathfrak{g}$-equivariant structure on $\mathcal{G}$, described locally by relations

$$
\begin{aligned}
& \mathscr{L}_{a} b=D_{2} \Upsilon_{a}, \\
& \mathscr{L}_{a} \Upsilon_{b}-\mathscr{L}_{b} \Upsilon_{a}-f_{a b c} \Upsilon_{c}=D_{1} \gamma_{a b}, \\
& \mathscr{L}_{a} \gamma_{b c}-\mathscr{L}_{b} \gamma_{a c}+\mathscr{L}_{c} \gamma_{a b}-f_{a b d} \gamma_{d c}+f_{a c d} \gamma_{d b}-f_{b c d} \gamma_{d a}=0 .
\end{aligned}
$$

A proof of the proposition is given in Appendix $\mathrm{E}$.

Along similar lines, we may rephrase Eq. (2.28) together with the constraint (3.7) in the presence of a defect in terms of a $\mathcal{G}$-twisted $\mathfrak{g}$-equivariant structure on the bi-brane.

Proposition 7.3. Let $M$ and $Q$ be a pair of G-spaces, equipped with a pair of smooth $\mathrm{G}$ equivariant maps $\iota_{\alpha}: Q \rightarrow M, \alpha=1,2$, and let $\mathcal{O}^{M}=\left\{\mathcal{O}_{i}^{M}\right\}_{i \in \mathscr{I}^{M}}$ and $\mathcal{O}^{Q}=\left\{\mathcal{O}_{i}^{Q}\right\}_{i \in \mathscr{I} Q}$ be the respective good open covers, chosen such that there exist Čech extensions $\check{\iota}_{\alpha}=\left(\iota_{\alpha}, \phi_{\alpha}\right)$. Furthermore, let $\mathcal{G}$ be a gerbe over $M$ with a $\mathrm{G}$-invariant curvature $\mathrm{H}$ and local data $b \in$ $A_{M}^{3}\left(\mathcal{O}^{M}\right)$. Suppose that $\mathcal{G}$ is endowed with a $\mathfrak{g}$-equivariant structure, understood in terms of its local data given by the $\left(\Upsilon_{a}, \gamma_{a b}\right)$ with the $\Upsilon_{a}$ and the $\gamma_{a b}$ as in Eqs. (7.8) and (7.9) of Proposition 7.9, respectively. Finally, let $p=\left(P_{i}, k_{i j}, r_{i j k}\right) \in A_{Q}^{2}\left(\mathcal{O}^{Q}\right)$ be local data of a 1-isomorphism $\Phi: \iota_{1}^{*} \mathcal{G} \stackrel{\cong}{\rightarrow} \iota_{2}^{*} \mathcal{G} \otimes I_{\omega}$ forming part of the structure of a $\mathcal{G}$-bi-brane $\mathcal{B}=$ $\left(Q, \iota_{1}, \iota_{2}, \omega, \Phi\right)$ with a $\mathrm{G}$-invariant curvature $\omega$, so that, in particular,

$$
D_{2} p=\check{\Delta}_{Q} b+\left(\left.\omega\right|_{\mathcal{O}_{i}^{Q}}, 0,0,0\right),
$$

where we have introduced the Čech-extended pullback operator

$$
\check{\Delta}_{Q}:=\check{\iota}_{2}^{*}-\check{\iota}_{1}^{*} .
$$

Given $k_{a} \in C^{\infty}(Q, \mathbb{R}), a=1,2, \ldots, \operatorname{dim} \mathfrak{g}$ that satisfy $E q .(2.28)$, write

$$
\Xi_{a}:=\left(-\left.k_{a}\right|_{\mathcal{O}_{i}^{Q}}+\imath_{a} P_{i}, 0\right) \in A_{Q}^{1}\left(\mathcal{O}^{Q}\right) .
$$


Relation (3.7) is satisfied iff there exists a $\mathcal{G}$-twisted $\mathfrak{g}$-equivariant structure on $\mathcal{B}$, described locally by relations

$$
\begin{aligned}
& \mathscr{L}_{a} p=\check{\Delta}_{Q} \Upsilon_{a}+D_{1} \Xi_{a}, \\
& \mathscr{L}_{a} \Xi_{B}-\mathscr{L}_{b} \Xi_{a}-f_{a b c} \Xi_{c}=-\check{\Delta}_{Q} \gamma_{a b} .
\end{aligned}
$$

A proof of the proposition is given in Appendix $\mathrm{F}$.

We conclude the present section by incorporating the remaining relation $(2.29)$ into the $\mathfrak{g}$-equivariance scheme.

Proposition 7.4. Let $(M, Q, T), T=\sqcup_{n \geq 3} T_{n}$ be a family of $\mathrm{G}$-spaces, equipped with smooth G-equivariant maps $\iota_{\alpha}: Q \rightarrow M, \alpha=1,2$ and $\pi_{n}^{k, k+1}: T_{n} \rightarrow Q, k=1,2, \ldots, n$, and let $\mathcal{O}^{M}=$ $\left\{\mathcal{O}_{i}^{M}\right\}_{i \in \mathscr{I}^{M}}, \mathcal{O}^{Q}=\left\{\mathcal{O}_{i}^{Q}\right\}_{i \in \mathscr{I} Q}$ and $\mathcal{O}^{T_{n}}=\left\{\mathcal{O}_{i}^{T_{n}}\right\}_{i \in \mathscr{I}^{T_{n}}}$ be the respective good open covers, chosen such that there exist Čech extensions $\check{\iota}_{\alpha}=\left(\iota_{\alpha}, \phi_{\alpha}\right)$ and $\check{\pi}_{n}^{k, k+1}=\left(\pi_{n}^{k, k+1}, \psi_{n}^{k, k+1}\right)$. Furthermore, let $\mathcal{G}$ be a gerbe over $M$ with a $\mathrm{G}$-invariant curvature $\mathrm{H}$ and local data $b \in$ $A_{M}^{3}\left(\mathcal{O}^{M}\right)$ and let $\mathcal{B}=\left(Q, \iota_{1}, \iota_{2}, \omega, \Phi\right)$ be a $\mathcal{G}$-bi-brane with a $\mathrm{G}$-invariant curvature $\omega$ and a 1-isomorphism $\Phi: \iota_{1}^{*} \mathcal{G} \stackrel{\cong}{\rightarrow} \iota_{2}^{*} \mathcal{G} \otimes I_{\omega}$ with local data $p \in A_{Q}^{2}\left(\mathcal{O}^{Q}\right)$. Suppose that both $\mathcal{G}$ and $\mathcal{B}$ are endowed with a $\mathfrak{g}$-equivariant structure, understood in terms of its local data given by the $\left(\Upsilon_{a}, \gamma_{a b}\right)$ as in Eqs. (7.8) and (7.9) of Proposition 7.8, and the $\Xi_{a}$ as in Eq. (7.13) of Proposition 7.3, respectively. Finally, let $h_{n}=\left(f_{n i}, q_{n i j}\right) \in A_{T_{n}}^{1}\left(\mathcal{O}^{T_{n}}\right)$ be local data of 2-isomorphisms of diagram (2.2) forming part of the structure of an $(\mathcal{G}, \mathcal{B})$-inter-bi-brane $\mathcal{J}=\left(T_{n},\left(\varepsilon_{n}^{k, k+1}, \pi_{n}^{k, k+1}\right) ; \varphi_{n}\right)$, so that, in particular,

$$
D_{1} h_{n}=-\check{\Delta}_{T_{n}} p
$$

where we have introduced the $\check{C}$ ech-extended pullback operator

$$
\check{\Delta}_{T_{n}}:=\sum_{k=1}^{n} \varepsilon_{n}^{k, k+1} \check{\pi}_{n}^{k, k+1 *} .
$$

Relation (2.29) is satisfied iff there exists a $\mathcal{B}$-twisted $\mathfrak{g}$-equivariant structure on $\mathcal{J}$, described locally by the relation

$$
\mathscr{L}_{a} h_{n}=-\check{\Delta}_{T_{n}} \Xi_{a} .
$$

A proof of the proposition is given in Appendix $\mathrm{G}$.

\section{G-EQuivariant String Backgrounds}

Our discussion in Section 1 of the circumstances in which the global gauge anomaly of the $\sigma$-model for arbitrary network-field configurations in the background of topologically trivial gauge fields vanishes revealed the necessity of endowing the string background $\mathfrak{B}=(\mathcal{M}, \mathcal{B}, \mathcal{J})$ with additional structure, composed of a 1-isomorphism $\Upsilon$ over $\mathrm{G} \times M$ and a 2-isomorphism $\Xi$ over $\mathrm{G} \times Q$, related as in Theorem 4.12. This additional structure can be viewed as the first component of a larger construct involving the geometric structure $(\mathcal{G}, \mathcal{B}, \mathcal{J})$ over the target space $\mathscr{F}=M \sqcup Q \sqcup T$ equipped with the action of a group $\mathrm{G}$ of rigid symmetries of the $\sigma$-model. The subsequent algebraic reinterpretation of conditions (3.6)-(3.8) in terms of a $\mathfrak{g}$-equivariant structure on $(\mathcal{G}, \mathcal{B}, \mathcal{J})$, provided in Section 7 , suggests already an extension of this first component to what we shall introduce below under the name of a G-equivariant string background. Its existence is a sufficient and rather natural condition for defining the gauged $\sigma$-model coupled to gauge fields in an arbitrary principal G-bundles over worldsheets, an issue discussed in Section 10. It is also suggested by the previous studies of orbifold and orientifold $\sigma$-models in Refs. GR03, Gaw05, SSW07, GSW08b, GSW08a dealing with discrete symmetries of the string background. Finally, it turns out to be a necessary and sufficient condition for the gauged $\sigma$-model to define a $\sigma$-model on the quotient $\mathscr{F} / \mathrm{G}$ (at least for smooth quotients $\mathscr{F} / \mathrm{G}$ ), as elaborated in Section 9 .

The definitions that make up this section rest heavily on the simplicial formalism and notation laid out in Section 6. Their physical significance will be substantiated in the remainder of the paper, in which the fundamental argument will be the descent principle discussed in the last part of the present section. 
8.1. G-equivariant structures. Let us start by recalling

Definition 8.1. GSW10, Def. 5.1] Let $\mathscr{M}$ be a G-space, and let G $\mathscr{M}$ be the nerve of the action groupoid $\mathrm{G} \ltimes \mathscr{M}$, with the corresponding face maps ${ }^{\mathscr{M}} d_{i}^{(n+1)}$, as introduced in Definition 2.18. Furthermore, let $\mathcal{G}$ be a gerbe over $\mathscr{M}$ with a G-invariant curvature $\mathrm{H}$ admitting a $\mathfrak{g}$-equivariantly closed G-equivariant (Cartan-model) extension $\widehat{\mathrm{H}}=\mathrm{H}-\kappa, \kappa \in \Omega^{1}(\mathscr{M}) \otimes \mathfrak{g}^{*}$. Finally, let $\rho$ be the 2 -form on $\mathrm{G} \times \mathscr{M}$ given in Eq. (2.30). A G-equivariant structure on gerbe $\mathcal{G}$ relative to 2 -form $\rho$ is a pair $(\Upsilon, \gamma)$ consisting of

- a 1-isomorphism

$$
\Upsilon: \mathscr{M}_{1}^{(1) *} \mathcal{G} \stackrel{\cong}{\rightrightarrows} d_{0}^{(1) *} \mathcal{G} \otimes I_{\rho}
$$

of gerbes over $\mathrm{G} \times \mathscr{M}$;

- a 2-isomorphism

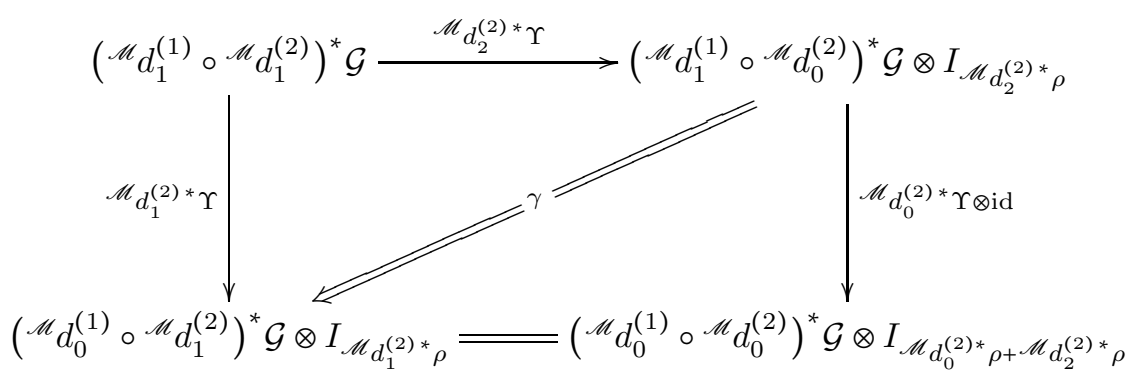

between the 1-isomorphisms over $\mathrm{G}^{2} \times \mathscr{M}$, satisfying, over $\mathrm{G}^{3} \times \mathscr{M}$, the coherence condition

$$
{ }^{\mathscr{M}} d_{1}^{(3) *} \gamma \bullet\left(\operatorname{id} \circ{ }^{\mathscr{M}} d_{3}^{(3) *} \gamma\right)={ }^{\mathscr{M}} d_{2}^{(3) *} \gamma \bullet\left(\left({ }^{\mathscr{M}} d_{0}^{(3) *} \gamma \otimes \mathrm{id}\right) \circ \mathrm{id}\right),
$$

equivalently expressed by the commutative 2-diagram (H.1) from Appendix $\mathbb{H}$.

Henceforth, the quadruple $(\mathcal{G}, \Upsilon, \gamma ; \kappa)$ will be called a gerbe G-equivariant relative to 2form $\rho$, or, simpler, a $(\mathrm{G}, \rho)$-equivariant gerbe, or, whenever there is no risk of confusion, just a G-equivariant gerbe.

Note that all the arrows in diagram (8.2) make sense in virtue of Eqs. (2.18) and (2.38).

Another useful notion is introduced in

Definition 8.2. GSW10, Sec. V.A] Let $\left(\mathcal{G}_{\beta}, \Upsilon_{\beta}, \gamma_{\beta} ; \kappa\right), \beta=1,2$ be a pair of G-equivariant gerbes of curvature $\mathrm{H}$ over a $\mathrm{G}$-space $\mathscr{M}$, as characterised in Definition 8.1. A G-equivariant 1-isomorphism between them is a pair $\left(\Psi_{1,2}, \eta_{1,2}\right)$ consisting of a 1-isomorphism

$$
\Psi_{1,2}: \mathcal{G}_{1} \stackrel{\cong}{\rightarrow} \mathcal{G}_{2}
$$

and of a 2-isomorphism

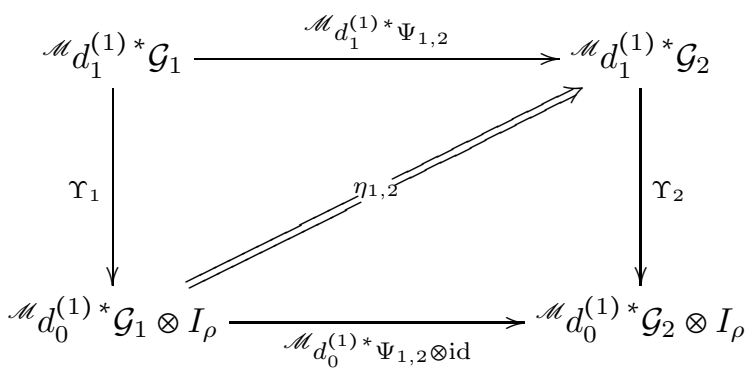

between the 1-isomorphisms over $\mathrm{G} \times \mathscr{M}$, satisfying, over $\mathrm{G}^{2} \times \mathscr{M}$, the coherence condition

$$
\mathscr{M}_{1}^{(2) *} \eta_{1,2} \bullet\left(\text { id } \circ \gamma_{1}\right)=\left(\gamma_{2} \circ \mathrm{id}\right) \bullet\left(\operatorname{id} \circ{ }^{\mathscr{M}} d_{2}^{(2) *} \eta_{1,2}\right) \bullet\left(\left({ }^{\mathscr{M}} d_{0}^{(2) *} \eta_{1,2} \otimes \mathrm{id}\right) \circ \mathrm{id}\right),
$$

equivalently expressed by the commutative 2-diagram (H.2) from Appendix $\mathrm{H}$.

The gerbes $\left(\mathcal{G}_{\beta}, \Upsilon_{\beta}, \gamma_{\beta} ; \kappa\right)$ will be called G-equivariantly 1-isomorphic. In the distinguished case of $\left(\mathcal{G}_{1}, \kappa_{1}\right)=\left(\mathcal{G}_{2}, \kappa_{2}\right)=:(\mathcal{G}, \kappa)$, we shall speak of equivalent G-equivariant structures on $\mathcal{G}$ relative to $\rho$. 
Once more, it is due to relations (2.18) and (2.38) that the above diagram is well-defined.

We complete the basic description of G-equivariant gerbes with

Definition 8.3. GSW10, Sec. V.A] Let $\left(\Psi_{1,2}^{\alpha}, \eta_{1,2}^{\alpha}\right), \alpha=1,2$ be a pair of G-equivariant 1-isomorphisms between two G-equivariant gerbes $\left(\mathcal{G}_{\beta}, \Upsilon_{\beta}, \gamma_{\beta} ; \kappa_{\beta}\right), \beta=1,2$ of curvature $\mathrm{H}$ over a G-space $\mathscr{M}$. A G-equivariant 2-isomorphism between them is a 2-isomorphism

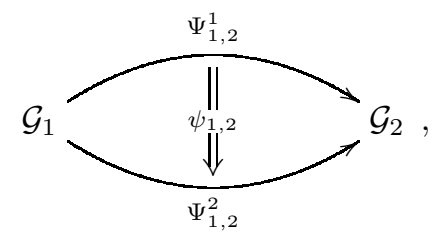

satisfying, over $\mathrm{G} \times \mathscr{M}$, the coherence condition

$$
\left(\mathrm{id} \circ{ }^{\mathscr{M}} d_{1}^{(1) *} \psi_{1,2}\right) \bullet \eta_{1,2}^{1}=\eta_{1,2}^{2} \bullet\left(\left({ }^{\mathscr{M}} d_{0}^{(1) *} \psi_{1,2} \otimes \mathrm{id}\right) \circ \mathrm{id}\right)
$$

equivalently expressed by the commutative 2-diagram (

In the next step, we extend the notion of G-equivariance to bi-branes.

Definition 8.4. Let $M, Q$ be a pair of G-spaces and let $\mathrm{G} \mathscr{M}, \mathscr{M}=M, Q$ be the respective nerves of the action groupoids $\mathrm{G} \ltimes \mathscr{M}$, with face maps $\mathscr{M}_{i}^{(n+1)}$, as introduced in Definition 2.18. Furthermore, let $(\mathcal{G}, \Upsilon, \gamma ; \kappa)$ be a $(\mathrm{G}, \rho)$-equivariant gerbe over $M$ as described in Definition 8.1. Consider a $\mathcal{G}$-bi-brane $\mathcal{B}=\left(Q, \iota_{\alpha}, \omega, \Phi\right)$ with $Q$ as a world-volume, and a pair of smooth G-maps $\iota_{\alpha}: Q \rightarrow M$. Suppose the curvature 2 -form $\omega$ is G-invariant and admits a G-equivariant (Cartan-model) extension $\widehat{\omega}=\omega-k, k \in \Omega^{0}(Q) \otimes \mathfrak{g}^{*}$ satisfying Eq. (3.27) and the relation

$$
\widehat{\mathrm{d}} \widehat{\omega}=-\Delta_{Q} \widehat{\mathrm{H}}
$$

in which $\Delta_{Q}=\iota_{2}^{*}-\iota_{1}^{*}$ and $\widehat{\mathrm{H}}$ is the G-equivariant extension of the curvature $\mathrm{H}$ of $\mathcal{G}$. Finally, let $\lambda$ be the 1 -form on $\mathrm{G} \times Q$ given in Eq. (2.31). A G-equivariant structure on $\mathcal{G}$-bi-brane $\mathcal{B}$ relative to $\mathbf{1}$-form $\lambda$ is a 2 -isomorphism

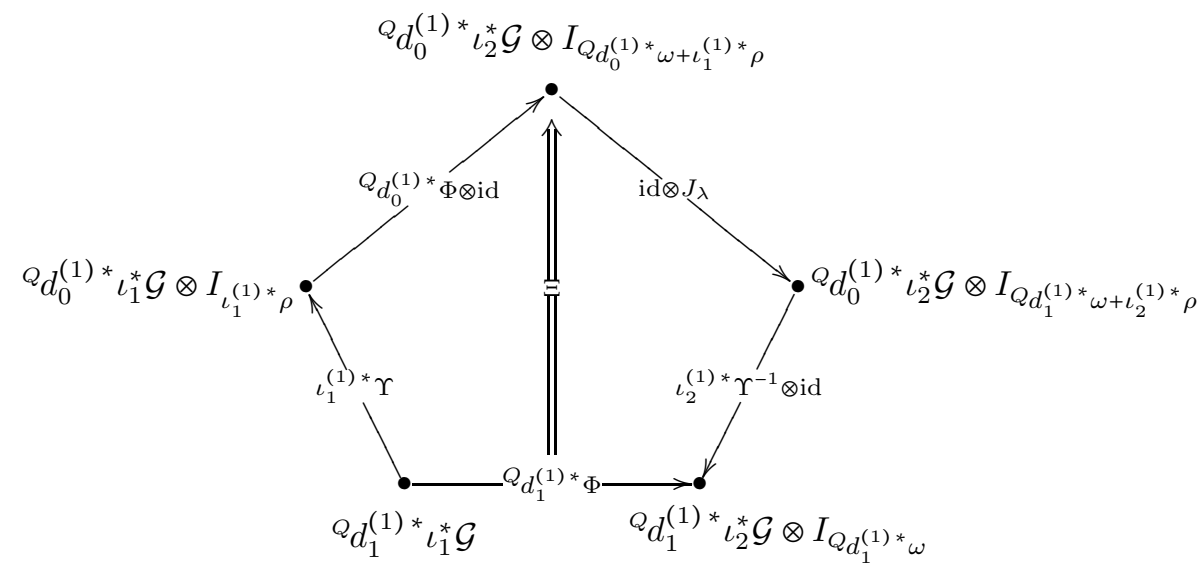

between the 1-isomorphisms over $\mathrm{G} \times Q$, subject to the coherence condition

$$
\left(\left(\iota_{2}^{(1) *} \gamma^{\sharp} \otimes \mathrm{id}\right) \circ \mathrm{id}\right) \bullet{ }^{Q} d_{1}^{(2) *} \Xi=\left(\mathrm{id} \circ \iota_{1}^{(1) *} \gamma\right) \bullet\left(\mathrm{id} \circ\left({ }^{Q} d_{0}^{(2) *} \Xi \otimes \mathrm{id}\right) \circ \mathrm{id}\right) \bullet{ }^{Q} d_{2}^{(2) *} \Xi,
$$

equivalently expressed by the commutative 2-diagram (H.4 from Appendix $\mathrm{H}$.

Henceforth, the triple $(\mathcal{B}, \Xi ; k)$ will be called a $(\mathcal{G}, \Upsilon, \gamma ; \kappa)$-bi-brane G-equivariant relative to 1-form $\lambda$, or, simpler, a $(\mathrm{G}, \lambda)$-equivariant $(\mathcal{G}, \Upsilon, \gamma ; \kappa)$-bi-brane, or, whenever there is no risk of confusion, just a G-equivariant $(\mathcal{G}$-) bi-brane.

In order to classify G-equivariant bi-branes, we employ 
Definition 8.5. Let $(\mathcal{G}, \Upsilon, \gamma ; \kappa)$ be a $(\mathrm{G}, \rho)$-equivariant gerbe over a $\mathrm{G}$-space $M$, as introduced in Definition 8.1, and let $\left(\mathcal{B}_{\beta}, \Xi_{\beta} ; k\right), \beta=1,2$ be a pair of $(\mathrm{G}, \lambda)$-equivariant $(\mathcal{G}, \Upsilon, \gamma ; \kappa)$-bi-branes, as described in Definition 8.4, with $\mathcal{B}_{\beta}=\left(Q, \iota_{\alpha}, \omega, \Phi_{\beta} \mid \alpha=1,2\right)$ (i.e. with the same world-volume, the same bi-brane maps $\iota_{\alpha}$ and of equal curvatures). We call the latter G-equivariantly equivalent iff the respective $\left(\iota_{1}^{*} \mathcal{G}, \iota_{2}^{*} \mathcal{G}\right)$-bi-modules $\Phi_{\beta}$ are G-equivariantly 2-isomorphic, that is iff there exists a 2-isomorphism

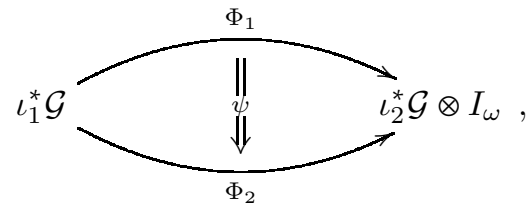

subject to the coherence condition

$$
\Xi_{2} \bullet{ }^{Q} d_{1}^{(1) *} \psi=\left({ }^{Q} d_{0}^{(1) *} \psi \otimes \mathrm{id}\right) \bullet \Xi_{1},
$$

equivalently expressed by the commutative 2-diagram (H.5) from Appendix H. Whenever $\Phi_{1}=\Phi_{2}=: \Phi$, we shall speak of equivalent G-equivariant structures on $\mathcal{G}$-bi-brane $\mathcal{B}=\left(Q, \iota_{1}, \iota_{2}, \omega, \Phi\right)$ relative to $\lambda$.

Finally, we pass to inter-bi-branes.

Definition 8.6. Let $M, Q$ and $T_{n}, n \geq 3$, be a collection of G-spaces and let G $\mathscr{M}, \mathscr{M}=$ $M, Q, T_{n}$, be the respective nerves of the action groupoids $G \ltimes \mathscr{M}$, with face maps ${ }^{\mathscr{M}} d_{i}^{(n+1)}$, as in Definition 2.18. Furthermore, let $(\mathcal{G}, \Upsilon, \gamma ; \kappa)$ be a $(\mathrm{G}, \rho)$-equivariant gerbe over $M$, and let $(\mathcal{B}, \Xi ; k)$ be a $(\mathrm{G}, \lambda)$-equivariant $(\mathcal{G}, \Upsilon, \gamma ; \kappa)$-bi-brane with $\mathcal{B}=\left(Q, \iota_{1}, \iota_{2}, \omega, \Phi\right)$ and with a $(\mathrm{G}, \lambda)$-equivariant $\left(\iota_{1}^{*} \mathcal{G}, \iota_{2}^{*} \mathcal{G}\right)$-bi-module $(\Phi, \Xi ; k)$. Finally, let $\mathcal{J}=\left(T_{n},\left(\varepsilon_{n}^{k, k+1}, \pi_{n}^{k, k+1}\right) ; \varphi_{n}\right)$ be a $(\mathcal{G}, \mathcal{B})$-inter-bi-brane with the $T_{n}$ as component world-volumes, and with smooth Gmaps $\pi_{n}^{k, k+1}: T_{n} \rightarrow Q$ and G-invariant orientation maps $\varepsilon_{n}^{k, k+1}: T_{n} \rightarrow\{-1,+1\}$. Suppose also that the 2-isomorphisms $\varphi_{n}$ satisfy the conditions

$$
\begin{aligned}
{ }_{n} d_{1}^{(1) *} \varphi_{n}= & \left(d_{\Upsilon}\right)_{n}^{1(1)} \bullet\left(\operatorname{id} \circ\left(i_{\Upsilon}^{\varepsilon^{1,2}}\right)_{n}^{1(1)}\right) \bullet\left(\mathrm{id} \circ \lambda_{\Upsilon_{n}^{1(1)}}\right) \bullet\left(\mathrm{id} \circ{ }^{T_{n}} d_{0}^{(1) *} \varphi_{n} \circ \mathrm{id}\right) \\
& \bullet\left(\operatorname{id} \circ \lambda_{\left(\Phi_{n}^{n, 1(1)}\right)_{2^{*}} \otimes \mathrm{id}} \circ \lambda_{\left(\Phi_{n}^{n-1, n}\right)_{2^{*}} \otimes \mathrm{id}} \circ \cdots \circ \lambda_{\left.\left(\Phi_{n}^{2,3}\right)_{2^{*} \otimes \mathrm{id}} \circ \mathrm{id}\right)}\right. \\
& \bullet\left(\operatorname{id} \circ\left(b_{\Upsilon}^{-1}\right)_{n}^{n(1)} \circ \mathrm{id} \circ\left(b_{\Upsilon}^{-1}\right)_{n}^{n-1(1)} \circ \mathrm{id} \circ \cdots \circ \mathrm{id} \circ\left(b_{\Upsilon}^{-1}\right)_{n}^{2(1)} \circ \mathrm{id}\right) \\
& \bullet\left(\operatorname{id} \circ\left(i_{\Upsilon}^{\varepsilon_{n}^{n, 1}}\right)_{n}^{n(1)} \circ \mathrm{id} \circ\left(i_{\Upsilon}^{\varepsilon_{n}^{n-1, n}}\right)_{n}^{n-1(1)} \circ \mathrm{id} \circ \cdots \circ \mathrm{id} \circ\left(i_{\Upsilon}^{\varepsilon_{n}^{2,3}}\right)_{n}^{2(1)} \circ \mathrm{id}\right) \\
& \bullet\left(\left(\Xi_{n}^{n, 1(1)} \otimes \mathrm{id}\right) \circ\left(\Xi_{n}^{n-1, n(1)} \otimes \mathrm{id}\right) \circ \cdots \circ \Xi_{n}^{1,2(1)}\right) .
\end{aligned}
$$

A $(\mathcal{G}, \mathcal{B})$-inter-bi-brane with these properties will be called a G-equivariant $((\mathcal{G}, \Upsilon, \gamma ; \kappa)$, $(\mathcal{B}, \Xi ; k)$ )-inter-bi-brane, or, whenever there is no risk of confusion, just a G-equivariant $((\mathcal{G}, \mathcal{B})$-)inter-bi-brane.

Altogether, we are naturally led to the following

Definition 8.7. A (G, $\rho, \lambda)$-equivariant string background is a string background whose target space carries the structure of a G-space, and such that the corresponding gerbe is $(\mathrm{G}, \rho)$-equivariant in the sense of Definition 8.1, the corresponding bi-brane is $(\mathrm{G}, \lambda)$ equivariant in the sense of Definition 8.4, and the corresponding inter-bi-brane is G-equivariant in the sense of Definition 8.6.

G-equivariant string backgrounds are among those that permit to consistently gauge the rigid G-symmetry of the $\sigma$-model on general world-sheets in the presence of topologically trivial world-sheet gauge fields in a manner that guarantees the absence of local and global gauge anomalies. Indeed, the former are absent in consequence of relations $(2.27)-(2.29)$ and (3.6)-(3.8), cf. Corollary 3.11, and the latter vanish due to the existence of the 1-isomorphism $\Upsilon$ and of the 2-isomorphism $\Xi$ with properties listed in Theorem 4.12. These conditions and properties are among those imposed by G-equivariance of the string background. In 
Section 10 below, we shall show that the full-blown G-equivariant structure carried by a string background actually enables us to define the gauged $\sigma$-model also in the topologically nontrivial sectors with the gauge fields that represent a connection in a nontrivial principal G-bundle over the world-sheet.

8.2. The descent principle for G-equivariant string backgrounds. The key feature of $(\mathrm{G}, \rho, \lambda)$-equivariant string backgrounds in the special case when $\rho=0=\lambda$ is that they descend to consistent string backgrounds on the quotient of the target space by the action of $\mathrm{G}$, provided the latter quotient is sufficiently regular. This fact will play a pivotal rôle in defining the topologically non-trivial sectors of the gauged $\sigma$-model on world-sheets containing a defect quiver.

We start by explaining the concept of 2-categorial descent. To this end, we consider

Definition 8.8. Given a surjective submersion $\varpi \mathscr{M}: \mathscr{M} \rightarrow \mathscr{X}$, and the associated simplicial space composed of fibred products of $\mathscr{M}$,

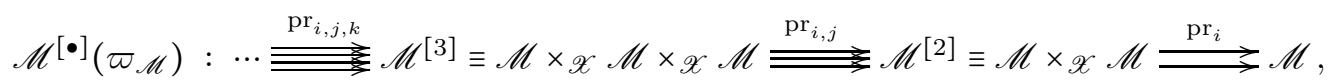

with face maps given by the canonical projections, the descent 2-category $\mathfrak{D e s}(\varpi \mathscr{M})$ is composed of

- the object class with elements given by triples $(\mathcal{G}, \Upsilon, \gamma)$, consisting of a gerbe $\mathcal{G}$ (with connection) over $\mathscr{M}$, a 1-isomorphism $\Upsilon: \mathcal{G}_{1^{*}} \stackrel{\cong}{\longrightarrow} \mathcal{G}_{2^{*}}$ over $\mathscr{M}^{[2]}$, and a 2-isomorphism

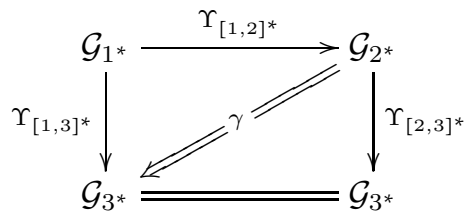

subject to the coherence condition

$$
\gamma_{[1,2,4]^{*}} \bullet\left(\gamma_{[2,3,4]^{*}} \circ \text { id }\right)=\gamma_{[1,3,4]^{*}} \bullet\left(\text { id } \circ \gamma_{[1,2,3]^{*}}\right),
$$

equivalently expressed by the commutative 2-diagram (H.6) from Appendix $\mathbb{H}$;

- for any pair $\mathcal{O}_{\alpha}=\left(\mathcal{G}_{\alpha}, \Upsilon_{\alpha}, \gamma_{\alpha}\right), \alpha=1,2$ of objects, a category $\mathfrak{H o m}\left(\mathcal{O}_{1}, \mathcal{O}_{2}\right)$, with

- objects (1-cells) given by pairs $(\Psi, \eta)$, consisting of a 1-isomorphism $\Psi: \mathcal{G}_{1} \stackrel{\cong}{\longrightarrow}$ $\mathcal{G}_{2}$ and a 2 -isomorphism

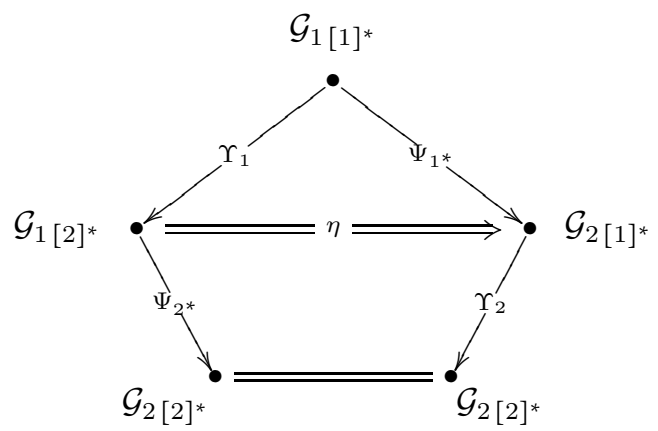

subject to the coherence condition

$$
\eta_{[1,3]^{*}} \bullet\left(\mathrm{id} \circ \gamma_{1}\right)=\left(\gamma_{2} \circ \mathrm{id}\right) \bullet\left(\mathrm{id} \circ \eta_{[1,2]^{*}}\right) \bullet\left(\eta_{[2,3]^{*}} \circ \mathrm{id}\right),
$$

equivalently expressed by the commutative 2-diagram (H.7) from Appendix $\mathbb{H}$;

- morphisms (2-cells) given by 2-isomorphisms

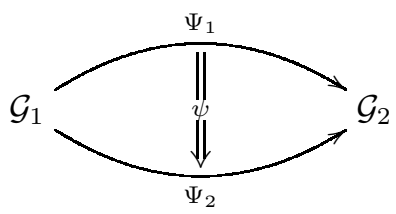


subject to the coherence condition

$$
\left(\text { id } \circ \psi_{1^{*}}\right) \bullet \eta_{1}=\eta_{2} \bullet\left(\psi_{2^{*}} \circ \mathrm{id}\right) \text {, }
$$

equivalently expressed by the commutative 2-diagram ( $\mathrm{H} .8)$ from Appendix $\mathbb{H}$.

The significance of the descent category manifests itself through the following

Theorem 8.9. Ste00, Prop. 6.7] GSW10, Thm. A.4.2] Given a surjective submersion $\varpi \mathscr{M}:$ $\mathscr{M} \rightarrow \mathscr{X}$, the induced pullback functor

$$
\widehat{\varpi}_{\mathscr{M}}^{*}: \mathfrak{B} \mathfrak{G r b} \nabla(\mathscr{X}) \rightarrow \mathfrak{D e s}(\varpi \mathscr{M}),
$$

defined by the formula

$\widehat{\varpi}_{\mathscr{M}}^{*} \mathcal{G}=\left(\varpi_{\mathscr{M}}^{*} \mathcal{G}, \mathrm{id}, \mathrm{id}\right), \quad \widehat{\varpi}_{\mathscr{M}}^{*}\left(\Psi: \mathcal{G}_{1} \stackrel{\cong}{\longrightarrow} \mathcal{G}_{2}\right)=\left(\varpi_{\mathscr{M}}^{*} \Psi, \mathrm{id}\right), \quad \widehat{\varpi}_{\mathscr{M}}^{*}\left(\psi: \Psi_{1} \cong \Psi_{2}\right)=\varpi_{\mathscr{M}}^{*} \psi$, is an equivalence of 2-categories.

For $\varpi_{\hat{\mathscr{M}}}: \hat{\mathscr{M}} \rightarrow \mathscr{M}$ a left principal G-bundle, the simplicial space $\hat{\mathscr{M}}^{[\bullet]}\left(\varpi_{\hat{\mathscr{M}}}\right)$ can be Gequivariantly identified with the nerve G $\hat{\mathscr{M}}$ of the action groupoid $\mathrm{G} \ltimes \hat{\mathscr{M}}$, and Definition 8.8 is readily seen to coincide with that of a sub-2-category $\mathfrak{B} \mathfrak{G} \mathfrak{r b}{ }^{\nabla}(\hat{\mathscr{M}})_{0}^{\mathrm{G}}$ of $(\mathrm{G}, 0)$-equivariant bundle gerbes with connection over $\hat{\mathscr{M}}$. As a simple corollary to Theorem 8.9, we then obtain

Theorem 8.10. GSW10, Thm. 5.3] Let $\varpi_{\hat{\mathscr{M}}}: \hat{\mathscr{M}} \rightarrow \mathscr{M}$ be a left principal G-bundle. There exists a canonical equivalence between the 2-category $\mathfrak{B} \mathfrak{G} \mathfrak{r b}{ }^{\nabla}(\mathscr{M})$ of bundle gerbes with connection over $\mathscr{M}=\hat{\mathscr{M}} / \mathrm{G}$ and the 2-category $\mathfrak{B} \mathfrak{G} \mathfrak{r b}{ }^{\nabla}(\hat{\mathscr{M}})_{0}^{\mathrm{G}}$ of $(\mathrm{G}, 0)$-equivariant bundle gerbes with connection over $\hat{\mathscr{M}}$.

Remark 8.11. The equivalence from Theorem 8.10 is preserved by pullback along Gequivariant maps.

Theorem 8.10 was proven in Ref. GSW10. Here, we want to study its consequences for bi-modules for G-equivariant gerbes and for their trivializations. We start with the former. We have the obvious

Corollary 8.12. Let $\varpi_{\hat{\mathscr{M}}}: \hat{\mathscr{M}} \rightarrow \mathscr{M}$ be a left principal G-bundle, and let $\hat{\mathcal{O}}_{\alpha}=\left(\hat{\mathcal{G}}_{\alpha}, \hat{\Upsilon}_{\alpha}, \hat{\gamma}_{\alpha}\right)$, $\alpha=1,2$, and the trivial gerbe $\hat{\mathcal{T}}=\left(I_{\hat{\omega}}, \hat{\mathcal{E}}, \hat{\varepsilon}\right)$, of an arbitrary curving $\hat{\omega} \in \Omega^{2}(\hat{\mathscr{M}})$ and $\mathrm{G}$ equivariant structure $(\hat{\mathcal{E}}, \hat{\varepsilon})$, all belong to the object class of the 2-category $\mathfrak{B} \mathfrak{G} \mathfrak{r} \mathfrak{b}^{\nabla}(\hat{\mathscr{M}})_{0}^{\mathrm{G}}$. Assume, furthermore, that there exists a 1-cell $(\hat{\Phi}, \hat{\Xi}) \in \mathfrak{H o m}\left(\hat{\mathcal{O}}_{1}, \hat{\mathcal{O}}_{2} \otimes \hat{\mathcal{T}}\right)$ in $\mathfrak{B} \mathfrak{G r b}{ }^{\nabla}(\hat{\mathscr{M}})_{0}^{\mathrm{G}}$. Then, there exist unique (up to 1-isomorphism) objects $\mathcal{G}_{1}, \mathcal{G}_{2}$ and $\mathcal{T}$ and a unique (up to 2isomorphism) 1-cell $\Phi: \mathcal{G}_{1} \rightarrow \mathcal{G}_{2} \otimes \mathcal{T}$ in the 2-category $\mathfrak{B} \mathfrak{G} \mathfrak{r b}{ }^{\nabla}(\mathscr{M})$ such that $\left(\varpi_{\hat{\mathscr{M}}}{ }^{\mathcal{G}_{\alpha}}, \mathrm{id}, \mathrm{id}\right)$ and $\left(\varpi_{\hat{\mathscr{M}}}^{*} \mathcal{T}, \mathrm{id}, \mathrm{id}\right)$ are G-equivariantly 1-isomorphic to $\hat{\mathcal{O}}_{\alpha}$ and $\hat{\mathcal{T}}$, respectively, and such that $\left(\varpi_{\hat{\mathscr{M}}}^{*} \Phi, \mathrm{id}\right)$ is G-equivariantly 2-isomorphic to $(\hat{\Phi}, \hat{\Xi})$.

Remark 8.13. The above corollary brings forward an issue that has to be resolved when formulating the $\sigma$-model in the presence of defects. The latter prerequires the structure of a bi-brane in the first place, cf. Ref. RS09, and so we need a characterisation of the circumstances in which the descent preserves the bi-brane structure, taking into account that $\mathcal{T}$ is not trivial in general. Thus, a bi-brane defined by a triple of $(\mathrm{G}, 0)$-equivariant gerbes $\hat{\mathcal{O}}_{\alpha}, \hat{\mathcal{T}}$ over a G-space $\hat{\mathscr{M}}$ does not always descend to a bi-brane over the quotient space $\mathscr{M}$. Clearly, the problem here lies with the arbitrary choice of the data $(\hat{\omega}, \hat{\mathcal{E}}, \hat{\mathcal{E}}$ ) (which appear to provide us with a most natural definition of a trivial $(\mathrm{G}, 0)$-equivariant gerbe on $\hat{\mathscr{M}}$ ). From the point of view of the construction of a consistent field theory, these data have to be further constrained.

The solution is contained in the following

Proposition 8.14. The equivalence of Theorem 8.10 restricts to an equivalence between, on the one hand, the sub-2-category $\mathfrak{T} \operatorname{riv} \mathfrak{G} \mathfrak{r b}(\hat{\mathscr{M}})_{0}^{\mathrm{G}}$ of $(\mathrm{G}, 0)$-equivariant gerbes over the $\mathrm{G}$-space $\hat{\mathscr{M}}$ G-equivariantly 1-isomorphic to $\mathrm{G}$-equivariant gerbes of the form $\left(I_{\hat{\omega}}, \mathrm{id}, \mathrm{id} ; 0\right)$, and, on the other hand, the sub-2-category $\mathfrak{T}$ rivGrb $\mathfrak{G}(\mathscr{M})$ of gerbes over $\mathscr{M}=\hat{\mathscr{M}} / \mathrm{G}$ 1-isomorphic to trivial gerbes. 
Proof. In the light of Theorem 8.10, the only thing that has to be checked is that every object in $\mathfrak{T} \mathfrak{r i v g} \mathfrak{G} \mathfrak{r}(\hat{\mathscr{M}})_{0}^{\mathrm{G}}$ descends to an object in $\mathfrak{T} \mathfrak{r i v G} \mathfrak{G} \mathfrak{b}(\mathscr{M})$, and that this mapping is essentially surjective, i.e. that each element of the latter 2-category can be obtained from an object of the former 2-category through descent. It is a straightforward exercise, using an explicit construction of the inverse of the functor $\widehat{\varpi}_{\mathscr{M}}^{*}$ given in Ref. Ste00], to verify that the gerbe over $\mathscr{M}$ associated to the trivial $(\mathrm{G}, 0)$-equivariant gerbe $\left(I_{\hat{\omega}}\right.$, id, id; 0$)$ over $\hat{\mathscr{M}}$ with a G-basic curving $\hat{\omega}$ is $I_{\omega}$, where $\omega$ is the unique 2 -form on $\mathscr{M}$ such that $\hat{\omega}=\varpi^{*} \hat{M}^{\omega}$. We also have, by construction, $\left(I_{\hat{\omega}}\right.$, id, id $\left.; 0\right)=\widehat{\varpi}^{*} I_{\omega}$.

Put together, Theorem 8.10, Remark 8.11 and Proposition 8.14 lead to a conclusion which is central to our subsequent considerations, and so we formulate it as

Theorem 8.15. Let $\varpi_{\hat{M}}: \hat{M} \rightarrow M$ and $\varpi_{\hat{Q}}: \hat{Q} \rightarrow Q$ be left principal G-bundles with the respective smooth bases $M=\hat{M} / \mathrm{G}$ and $Q=\hat{Q} / \mathrm{G}$. There exists a canonical bijection

$$
\left\{\begin{array}{c}
\text { G-equivariant isomorphism classes } \\
\text { of }(\mathrm{G}, 0) \text {-equivariant bi-branes } \\
\text { with world-volume } \hat{Q} \\
\text { for }(\mathrm{G}, 0) \text {-equivariant gerbes over } \hat{M}
\end{array}\right\} \stackrel{1: 1}{\longleftrightarrow} \quad\left\{\begin{array}{c}
\text { Equivalence classes } \\
\text { of bi-branes } \\
\text { with world-volume } Q \\
\text { for gerbes over } M
\end{array}\right\} \text {. }
$$

Proof. Let us begin by identifying the bi-brane with world-volume $Q$ associated to the given (G,0)-equivariant bi-brane $(\hat{\mathcal{B}}, \Xi ; 0)$ with $\hat{\mathcal{B}}=\left(\hat{Q}, \hat{\iota}_{1}, \hat{\iota}_{2}, \hat{\omega}, \hat{\Phi}\right)$ for a $(\mathrm{G}, 0)$-equivariant gerbe $\hat{\mathcal{G}}$ over $\hat{M}$. First of all, note that the G-equivariant bi-brane maps $\hat{\iota}_{\alpha}: \hat{Q} \rightarrow \hat{M}$ induce smooth maps $\bar{\iota}_{\alpha}: Q \rightarrow M$ which render the following diagram commutative:

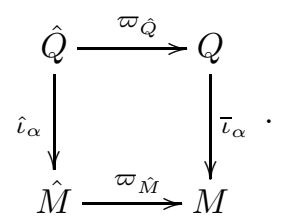

It is these quotient maps that enter the definition of the bi-brane over $Q$. Second, the vanishing of $\rho$ and $\lambda$ implies $\kappa_{a}=0$ and $k_{a}=0$, and so, in virtue of Eq. (2.28), we obtain

$$
\imath_{a} \hat{\omega}=0 .
$$

This, in conjunction with the assumed G-invariance of $\hat{\omega}$, shows that the latter 2-form is, in fact, G-basic, hence, in particular, there exists a unique 2-form $\omega \in \Omega^{2}(Q)$ such that

$$
\hat{\omega}=\varpi_{\hat{Q}}^{*} \omega .
$$

Next, we examine the bi-brane 1-isomorphism $\hat{\Phi}$. Let $\mathcal{G}$ be the gerbe over $M$ obtained from the $(\mathrm{G}, 0)$-equivariant gerbe $(\hat{\mathcal{G}}, \hat{\Upsilon}, \hat{\gamma} ; 0)$ according to Theorem 8.10. Owing to the assumed G-equivariance of the $\hat{\iota}_{\alpha}$, the pullback gerbes $\left(\hat{\iota}_{\alpha}^{*} \hat{\mathcal{G}}, \hat{\iota}_{\alpha}^{(1) *} \Upsilon, \hat{\iota}_{\alpha}^{(2) *} \gamma ; 0\right)$ descend, in conformity with the same theorem, to the respective gerbes $\bar{\iota}_{\alpha}^{*} \mathcal{G}$ over $Q$. Finally, also $\left(I_{\hat{\omega}}, \mathrm{id}, \mathrm{id} ; 0\right)$ with the G-basic curving $\hat{\omega}=\varpi_{\hat{Q}}^{*} \omega$ descends to the trivial gerbe $I_{\omega}$. Theorem 8.10 now predicts the existence of a descendant 1-isomorphism

$$
\Phi: \bar{\iota}_{1}^{*} \mathcal{G} \stackrel{\cong}{\longrightarrow} \bar{\iota}_{2}^{*} \mathcal{G} \otimes I_{\omega}
$$

between the respective descendant gerbes, and thus yields the desired descendant bi-module. Clearly, G-equivariantly equivalent $(\mathrm{G}, 0)$-equivariant bi-branes over $\hat{Q}$ are mapped to equivalent ones over $Q$.

For the reverse statement, note that the 2 -isomorphism class of a bi-module $\Phi: \bar{\iota}_{1}^{*} \mathcal{G} \stackrel{\cong}{\longrightarrow}$ $\bar{\iota}_{2}^{*} \mathcal{G} \otimes I_{\omega}$ over $Q$ for a gerbe $\mathcal{G}$ over $M$ and with $\omega \in \Omega^{2}(Q)$ corresponds to the G-equivariant 2-isomorphism class of the $(\mathrm{G}, 0)$-equivariant bi-module $\hat{\Phi}=\varpi_{\hat{Q}}^{*} \Phi$, with

$$
\hat{\Phi}: \varpi_{\hat{Q}}^{*} \bar{l}_{1}^{*} \mathcal{G} \stackrel{\cong}{\longrightarrow} \varpi_{\hat{Q}}^{*} \bar{l}_{2}^{*} \mathcal{G} \otimes I_{\varpi}^{*} \omega .
$$

The commutativity of diagram (8.14) then enables us to rewrite the above as

$$
\hat{\Phi}: \hat{\iota}_{1}^{*} \varpi_{\hat{M}}^{*} \mathcal{G} \stackrel{\cong}{\longrightarrow} \hat{\iota}_{2}^{*} \varpi_{\hat{M}}^{*} \mathcal{G} \otimes I_{\varpi}^{*} \omega
$$


and the gerbe $\varpi_{\hat{M}}^{*} \mathcal{G}$ over $\hat{M}$ is manifestly $(\mathrm{G}, 0)$-equivariant as the functorial image (with respect to the equivalence $\widehat{\varpi}_{\hat{M}}^{*}$ ) of the gerbe $\mathcal{G}$ entering the definition of $\Phi$.

Remark 8.16. The one-to-one correspondence of Theorem 8.15 is preserved by pullback along G-equivariant maps.

It is equally straightforward to prove

Theorem 8.17. Let $\left(\hat{M}, \varpi_{\hat{M}}\right),\left(\hat{Q}, \varpi_{\hat{Q}}\right)$ and $\left(\hat{T}_{n}, \varpi_{\hat{T}_{n}}\right), n \geq 3$, be principal G-manifolds with the respective smooth bases $M=\hat{M} / \mathrm{G}, Q=\hat{Q} / \mathrm{G}$ and $T_{n}=\hat{T}_{n} / \mathrm{G}$. There exists a canonical bijection

$$
\left\{\begin{array}{c}
\text { G-equivariant inter-bi-branes } \\
\text { with world-volume } \hat{T}=\bigsqcup_{n \geq 3} \hat{T}_{n} \\
\text { for }(\mathrm{G}, 0) \text {-equivariant bi-branes } \\
\text { with world-volume } \hat{Q} \\
\text { for gerbes over } \hat{M}
\end{array}\right\} \stackrel{1: 1}{\longleftrightarrow}\left\{\begin{array}{c}
\text { Inter-bi-branes } \\
\text { with world-volume } T=\sqcup_{n \geq 3} T_{n} \\
\text { for bi-branes with world-volume } Q \\
\text { for gerbes over } M
\end{array}\right\} \text {. }
$$

Proof. Consider a G-equivariant inter-bi-brane $\hat{\mathcal{J}}=\left(\hat{T}_{n},\left(\hat{\varepsilon}_{n}^{k, k+1}, \hat{\pi}_{n}^{k, k+1}\right) ; \hat{\varphi}_{n}\right)$ for a $(\mathrm{G}, 0)$ equivariant bi-brane $(\hat{\mathcal{B}}, \hat{\Xi} ; 0)$ with $\hat{\mathcal{B}}=\left(\hat{Q}, \hat{\iota}_{\alpha}, \hat{\omega}, \hat{\Phi}\right)$. The G-equivariant maps $\hat{\pi}_{n}^{k, k+1}: \hat{T}_{n} \rightarrow$ $\hat{Q}$ induce smooth maps $\bar{\pi}_{n}^{k, k+1}: T_{n} \rightarrow Q$ which render the following diagram commutative:

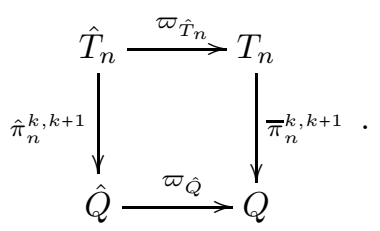

We shall employ the induced maps to explicitly construct the descendant inter-bi-brane $\mathcal{J}=\left(T_{n},\left(\bar{\varepsilon}_{n}^{k, k+1}, \bar{\pi}_{n}^{k, k+1}\right), \varphi_{n}\right)$ for $\hat{\mathcal{J}}$. The descendant inter-bi-brane has the orientation maps $\bar{\varepsilon}_{n}^{k, k+1}$ trivially induced from the respective orientation maps $\hat{\varepsilon}_{n}^{k, k+1}$, which makes sense as the G-action preserves each of the component world-volumes of $\hat{\mathcal{J}}$ corresponding to fixed values of the $\hat{\varepsilon}_{n}^{k, k+1}$. It therefore remains to identify the descendant 2-isomorphisms $\varphi_{n}$. Let $\Phi$ be the gerbe bi-module over $Q$ obtained from $\hat{\Phi}$ through Theorem 8.15. The maps $\hat{\pi}_{n}^{k, k+1}$ being G-equivariant, the 1-isomorphisms $\hat{\Phi}_{n}^{k, k+1}$ descend, in virtue of the same theorem, to the respective 1-isomorphisms $\Phi_{n}^{k, k+1}=\bar{\pi}_{n}^{k, k+1 *} \Phi^{\bar{\varepsilon}_{n}^{k, k+1}}$ over $T_{n}$, and so also the composite 1-isomorphism $\left(\hat{\Phi}_{n}^{n, 1} \otimes \mathrm{id}\right) \circ\left(\hat{\Phi}_{n}^{n-1, n} \otimes \mathrm{id}\right) \circ \cdots \circ \hat{\Phi}_{n}^{1,2}$ descends to the 1-isomorphism $\left(\Phi_{n}^{n, 1} \otimes \mathrm{id}\right) \circ\left(\Phi_{n}^{n-1, n} \otimes \mathrm{id}\right) \circ \cdots \circ \Phi_{n}^{1,2}$. Invoking Theorem 8.10, we then find descendant 2isomorphisms

$$
\varphi_{n}:\left(\Phi_{n}^{n, 1} \otimes \mathrm{id}\right) \circ\left(\Phi_{n}^{n-1, n} \otimes \mathrm{id}\right) \circ \cdots \circ \Phi_{n}^{1,2} \stackrel{\cong}{\Longrightarrow} \operatorname{id}_{\mathcal{G}_{n}^{1}}
$$

between the respective descendant 1-isomorphisms, the latter of them being written for the descendant gerbe $\mathcal{G}_{n}^{1}=\left(\bar{\iota}_{1}^{\bar{\varepsilon}_{n}^{1,2}} \circ \bar{\pi}_{n}^{1,2}\right)^{*} \mathcal{G}$, as dictated by the same theorem for the G-equivariant $\operatorname{map} \hat{\iota}_{1}^{1,2} \circ \hat{\pi}_{n}^{1,2}$.

Conversely, 2-isomorphisms $\varphi_{n}:\left(\Phi_{n}^{n, 1} \otimes \mathrm{id}\right) \circ\left(\Phi_{n}^{n-1, n} \otimes \mathrm{id}\right) \circ \cdots \circ \Phi_{n}^{1,2} \stackrel{\cong}{\Longrightarrow} \mathrm{id}_{\mathcal{G}_{n}^{1}}$ trivialising the concatenations of 1-isomorphisms $\Phi_{n}^{k, k+1}$ for the gerbe $\mathcal{G}$ over $M$, pulled back from $Q$ along the respective maps $\bar{\pi}_{n}^{k, k+1}$ as described above, yield the manifestly G-equivariant 2-isomorphisms $\hat{\varphi}_{n}=\varpi_{\hat{T}_{n}}^{*} \varphi_{n}$, with

$$
\hat{\varphi}_{n}:\left(\left(\varpi_{\hat{T}_{n}}^{*} \Phi_{n}^{n, 1} \otimes \mathrm{id}\right) \circ\left(\varpi_{\hat{T}_{n}}^{*} \Phi_{n}^{n-1, n} \otimes \mathrm{id}\right) \circ \cdots \circ \varpi_{\hat{T}_{n}}^{*} \Phi_{n}^{1,2} \stackrel{\cong}{=} \operatorname{id}_{\varpi_{\hat{T}_{n}}^{*}} \mathcal{G}_{n}^{1} .\right.
$$

Repeated application of the commutative diagram (8.16) enables to rewrite the above in the sought-after form

$$
\hat{\varphi}_{n}:\left(\left(\varpi_{\hat{Q}}^{*} \Phi\right)_{n}^{n, 1} \otimes \mathrm{id}\right) \circ\left(\left(\varpi_{\hat{Q}}^{*} \Phi\right)_{n}^{n-1, n} \otimes \mathrm{id}\right) \circ \cdots \circ\left(\varpi_{\hat{Q}}^{*} \Phi\right)_{n}^{1,2} \stackrel{\cong}{=} \operatorname{id}_{\left(\varpi_{M}^{*} \mathcal{G}\right)_{n}^{1}},
$$

with $\left(\varpi_{\hat{M}}^{*} \mathcal{G}\right)_{n}^{1}=\left(\hat{\iota}_{1}^{1,2} \circ \hat{\pi}_{n}^{1,2}\right)^{*} \varpi_{\hat{M}}^{*} \mathcal{G}$ and $\varpi_{\hat{Q}}^{*} \Phi$ manifestly $(\mathrm{G}, 0)$-equivariant. 
We may summarise the findings of the present section in

Corollary 8.18. Let $\hat{\mathscr{F}}=\hat{M} \sqcup \hat{Q} \sqcup \hat{T}$ be the target space of a string background, endowed with the structure of a $\mathrm{G}$-space with respect to a (left) action of the group $\mathrm{G}$, the latter assumed such that the canonical projection $\hat{\mathscr{F}} \rightarrow \hat{\mathscr{F}} / \mathrm{G}=: \mathscr{F}$ gives a principal $\mathrm{G}$-bundle. There exists a canonical bijection

$$
\left\{\begin{array}{c}
\text { Equivalence classes } \\
\text { of }(\mathrm{G}, 0,0) \text { - equivariant string backgrounds } \\
\text { with target space } \hat{\mathscr{F}}
\end{array}\right\} \stackrel{1: 1}{\longleftrightarrow}\left\{\begin{array}{c}
\text { Equivalence classes } \\
\text { of string backgrounds } \\
\text { with target space } \mathscr{F}
\end{array}\right\} \text {. }
$$

\section{Coset $\sigma$-MODELS}

One of the physical ideas underlying the concept of a gauged $\sigma$-model, particularly amply illustrated on the example of the (gauged) WZW models and the coset models of conformal field theory, cf. Refs. GK89b, GK89a, KPSY89, is that promoting global symmetries of the theory to the rank of local ones may serve to reduce the number of field-theoretic degrees of freedom. This systematic construction circumvents the usual problems posed by attempts at defining the two-dimensional field theory directly on the quotient $\mathscr{F} / \mathrm{G}$ of the original target space $\mathscr{F}$, such as metric singularities and the emergence of another component of the string background, that is the dilaton. It does so in a manner analogous to that in which G-equivariant cohomology of a G-space $\mathscr{M}$ replaces the cohomology of the orbit space of $\mathscr{M}$ in circumstances in which the orbit space is no longer smooth, that is by giving us a field theory which would, in the case of smooth $\mathscr{F} / \mathrm{G}$, be a 'pullback' to $\mathscr{F}$ (in a sense that shall be clarified below) of a unique field theory on the quotient. Below, we shall disregard - for the sake of illustration - the possible problems of the latter field theory mentioned above and explicitly carry out the descent from the field space $\mathscr{F}$ to its quotient, which boils down to integrating out the (non-dynamical) gauge field. This will make apparent the rôle of the G-equivariant structure on the string background.

We begin our discussion by remarking that the very structure of the action functional of Corollary 3.17, at most quadratic in the world-sheet gauge field $\mathrm{A}_{u}^{a}$, indicates that the effective two-dimensional field theory obtained by integrating out the gauge field in the path integral of the gauged $\sigma$-model can be read off, at least up to a dilaton term induced from the gaussian path integral over the gauge fields, from Eq. (3.30) upon solving the classical equations for $\mathrm{A}_{u}^{a}$ and subsequently substituting the solution back into the action functional, cf. Ref. RS09, Sect. 2.4].

In the conformal gauge of the world-sheet metric, the field equations for the gauge field can be written as

$$
\left(\begin{array}{cc}
\mathrm{h}_{a b} & \mathrm{ic}_{a b} \\
-\mathrm{ic}_{a b} & \mathrm{~h}_{a b}
\end{array}\right)\left(\begin{array}{c}
\mathrm{A}_{1}^{b} \\
\mathrm{~A}_{2}^{b}
\end{array}\right)=\left(\begin{array}{cc}
\mathrm{K}_{a \mu} & -\mathrm{i} \kappa_{a \mu} \\
\mathrm{i} \kappa_{a \mu} & \mathrm{K}_{a \mu}
\end{array}\right)\left(\begin{array}{l}
\partial_{1} \varphi^{\mu} \\
\partial_{2} \varphi^{\mu}
\end{array}\right)
$$

(in terms of the 1-forms $\mathrm{K}_{a}$ of Eq. (3.18) and of the symmetric tensor $\mathrm{h}_{a b}$ of Eq. (3.19)) and

$$
k_{a}=0 .
$$

In order to be able to solve the former, we need to make certain assumptions about the geometry of the (metric) target space $(M, \mathrm{~g})$. Namely, we shall assume that $M \stackrel{\varpi_{M}}{\longrightarrow} M / \mathrm{G}$ carries the structure of a principal G-bundle, so that, in particular, the G-action on $M$ is free and $g$ defines a positive-definite metric on the fibres of $M$. From the last fact, it then follows that the symmetric matrix $h$ with entries $h_{a b}$ is invertible. It is now easy to see that the background matrix

$$
\mathrm{E}:=\mathrm{h}-\mathrm{ch}^{-1} \mathrm{c}
$$

is invertible as well. Indeed, the latter can be rewritten as $h\left(1-h^{-1} c\right)\left(1+h^{-1} c\right)$, and so its invertibility is ensured by the absence of eigenvectors of the matrix $h^{-1} c$ associated with the eigenvalues \pm 1 . Under the above assumptions, we obtain

$$
\mathrm{A}_{u}^{a}=\mathrm{M}_{\mu}^{a}(\varphi) \partial_{u} \varphi^{\mu}-\mathrm{i} \varepsilon_{u v} \mathrm{~N}_{\mu}^{a}(\varphi) \partial_{v} \varphi^{\mu},
$$

with

$$
\mathrm{M}_{\mu}^{a}:=\left(\mathrm{E}^{-1}\right)^{a b} \mathrm{~K}_{b \mu}+\left(\mathrm{h}^{-1} \mathrm{cE}^{-1}\right)^{a b} \kappa_{b \mu}, \quad \mathrm{N}_{\mu}^{a}:=\left(\mathrm{E}^{-1}\right)^{a b} \kappa_{b \mu}+\left(\mathrm{h}^{-1} \mathrm{cE}^{-1}\right)^{a b} \mathrm{~K}_{b \mu} .
$$


The effective field theory, defined by the Feynman amplitudes

$$
\mathscr{A}^{\mathrm{eff}}[(\varphi \mid \Gamma)]:=\int \mathscr{D} \mathrm{A} \mathscr{A}[(\varphi \mid \Gamma) ; \mathrm{A}, \gamma]
$$

takes the form of a non-linear $\sigma$-model

$$
\mathscr{A}^{\mathrm{eff}}[(\varphi \mid \Gamma)]=\mathrm{e}^{-\frac{1}{2} \int_{\Sigma} \underline{\mathrm{g}}\left(\mathrm{d} \varphi \hat{\wedge} \star_{\gamma} \mathrm{d} \varphi\right)-W_{\mathrm{di} i}[\varphi]} \operatorname{Hol}_{\underline{\mathcal{G}}, \underline{\Phi}, \underline{\varphi}_{n}}(\varphi),
$$

with $W_{\text {dil }}$ the induced dilaton term (to be left unspecified), and with a new background $\underline{\mathfrak{B}}$ composed of

- a new target $\underline{\mathcal{M}}=(M, \underline{\mathrm{g}}, \underline{\mathcal{G}})$ with the metric

$$
\underline{\mathrm{g}}:=\mathrm{g}+\mathrm{E}^{-1 a b}\left(\kappa_{a} \otimes \kappa_{b}-\mathrm{K}_{a} \otimes \mathrm{K}_{b}\right)+\left(\mathrm{h}^{-1} \mathrm{c} \mathrm{E}^{-1}\right)^{a b}\left(\kappa_{a} \otimes \mathrm{K}_{b}+\mathrm{K}_{b} \otimes \kappa_{a}\right),
$$

and the gerbe

$$
\underline{\mathcal{G}}:=\mathcal{G} \otimes I_{\beta},
$$

where the curving $\beta \in \Omega^{2}(M)$ of the trivial factor reads

$$
\beta=\frac{1}{2}\left(\mathrm{~h}^{-1} \mathrm{cE}^{-1}\right)^{a b}\left(\kappa_{a} \wedge \kappa_{b}-\mathrm{K}_{a} \wedge \mathrm{K}_{b}\right)+\mathrm{E}^{-1 a b} \kappa_{a} \wedge \mathrm{K}_{b} ;
$$

- a new bi-brane $\underline{\mathcal{B}}=\left(\underline{Q}, \iota_{\alpha}, \underline{\omega}, \underline{\Phi} \mid \alpha=1,2\right)$ of world-volume, assumed smooth,

$$
\underline{Q}:=\bigcap_{a=1}^{\operatorname{dim} \mathfrak{g}} k_{a}^{-1}(\{0\})
$$

the curvature

$$
\underline{\omega}:=\omega-\Delta_{Q} \beta
$$

and with the bi-brane 1-isomorphism

$$
\underline{\Phi}:=\Phi \otimes \operatorname{id}_{I_{\iota_{1}^{*} \beta}^{*}}: \iota_{1}^{*} \underline{\mathcal{G}} \stackrel{\cong}{\longrightarrow} \iota_{2}^{*} \underline{\mathcal{G}} \otimes I_{\underline{\omega}},
$$

the latter two pulled back to $Q$;

- a new inter-bi-brane $\underline{\mathcal{J}}=\left(\underline{T}_{n},\left(\varepsilon_{n}^{k, k+1}, \pi_{n}^{k, k+1}\right) ; \underline{\varphi}_{n}\right)$ of the component world-volumes, assumed smooth,

$$
\underline{T}_{n}:=\bigcap_{k=1}^{n}\left(\pi_{n}^{k, k+1}\right)^{-1}(\underline{Q})
$$

and with the inter-bi-brane 2-isomorphisms

$$
\underline{\varphi}_{n}:=\varphi_{n} \otimes \operatorname{id}_{\operatorname{id}_{I_{n}^{1}}} .
$$

We shall next study the symmetry properties of the new string background with respect to the (induced) action of the group G.

First, we consider the target-space structures, i.e. the metric $\underline{g}$ and the gerbe $\underline{\mathcal{G}}$. We find

Proposition 9.1. Let $\varpi_{M}: M \rightarrow M / \mathrm{G}$ be a left principal $\mathrm{G}$-bundle with a $\mathrm{G}$-invariant metric $\mathrm{g}$, and with a $\mathrm{G}$-invariant closed 3-form $\mathrm{H}$ admitting a $\mathfrak{g}$-equivariantly closed extension $\widehat{\mathrm{H}}=\mathrm{H}-\kappa$ for $\kappa \in \Omega^{1}(M) \otimes \mathfrak{g}^{*}$. The metric $\mathrm{g}$ introduced in Eq. (9.2) is $\mathrm{G}$-basic, and so there exists a unique metric $\underline{\mathrm{G}}$ on $M / \mathrm{G}$ such that

$$
\underline{\mathrm{g}}=\varpi_{M}^{*} \underline{\mathrm{G}} .
$$

Proof. Using the identities

$$
\left(\mathrm{cE}^{-1}\right)^{a b}=-\left(\mathrm{E}^{-1} \mathrm{c}\right)^{b a}, \quad \mathrm{~h}^{-1} \mathrm{cE}^{-1} \mathrm{~h}=\mathrm{E}^{-1} \mathrm{c},
$$

we readily verify the $\mathfrak{g}$-horizontality of $\underline{\mathrm{g}}$,

$$
\begin{aligned}
\underline{\mathrm{g}}\left({ }^{M} \mathscr{K}_{a}, \cdot\right) & =\mathrm{K}_{a}+\mathrm{E}^{-1 b c}\left(\mathrm{c}_{a b} \kappa_{c}-\mathrm{h}_{a b} \mathrm{~K}_{c}\right)+\left(\mathrm{h}^{-1} \mathrm{cE}^{-1}\right)^{b c}\left(\mathrm{c}_{a b} \mathrm{~K}_{c}+\mathrm{h}_{a c} \kappa_{b}\right) \\
& =\left(\mathbf{1}-\mathrm{hE}^{-1}+\mathrm{ch}^{-1} \mathrm{cE} \mathrm{E}^{-1}\right)^{a b} \mathrm{~K}_{b}+\left(\mathrm{h}^{-1} \mathrm{cE}^{-1} \mathrm{~h}-\mathrm{E}^{-1} \mathrm{c}\right)^{b a} \kappa_{b}=0 .
\end{aligned}
$$


Its G-invariance is a consequence of the same property of $\mathrm{g}$, as well as of the G-equivariance of $\kappa$ and that of the fundamental vector fields,

$$
\left({ }^{M} \ell_{g}\right)_{*}{ }^{M} \mathscr{K}_{a}=\left(\operatorname{Ad}_{g}\right)_{a b}{ }^{M} \mathscr{K}_{b} .
$$

The last identity readily follows from Eq. (2.35).

Similarly, we establish the symmetry properties of the 2 -form $\beta$, summarised in

Proposition 9.2. Under the assumptions and in the notation of Proposition 9.1, the 2-form $\beta$ introduced in Eq. (9.4) is G-invariant and satisfies the identity

$$
\imath_{a} \beta=-\kappa_{a}
$$

Consequently, the curvature

$$
\underline{\mathrm{H}}:=\mathrm{H}+\mathrm{d} \beta
$$

of the gerbe $\underline{\mathcal{G}}$ defined in Eq. (9.3) is G-basic, and so there exists a unique 3-form $\underline{\mathrm{h}}$ on $M / \mathrm{G}$ such that

$$
\underline{\mathrm{H}}=\varpi_{M}^{*} \underline{\mathrm{h}} .
$$

Proof. The G-invariance of $\beta$ follows as before. Identity 9.10 is now obtained as

$$
\begin{aligned}
\imath_{a} \beta & =\left(\mathrm{h}^{-1} \mathrm{cE}^{-1}\right)^{b c}\left(\mathrm{c}_{a b} \kappa_{c}-\mathrm{h}_{a b} \mathrm{~K}_{c}\right)+\mathrm{E}^{-1 b c}\left(\mathrm{c}_{a b} \mathrm{~K}_{c}-\mathrm{h}_{a c} \kappa_{b}\right) \\
& =\left(\left(\mathrm{ch}^{-1} \mathrm{c}-\mathrm{h}\right) \mathrm{E}^{-1}\right)^{a b} \kappa_{b}=-\kappa_{a},
\end{aligned}
$$

using the symmetry of the background matrix. Put together, the above identities yield

$$
\imath_{a} \mathrm{~d} \beta=-\mathrm{d}\left(\imath_{a} \beta\right)=\mathrm{d} \kappa_{a},
$$

whence also

$$
\imath_{a} \underline{\mathrm{H}}=-\mathrm{d} \kappa_{a}+\mathrm{d} \kappa_{a}=0 .
$$

Since $\underline{\mathrm{H}}$ is also G-invariant, it is G-basic.

At this point, it seems pertinent to enquire as to the circumstances under which the new gerbe $\underline{\mathcal{G}}$ descends to the quotient $M / \mathrm{G}$ in the sense of Section 8.2. The answer is provided by

Proposition 9.3. Let $\varpi_{M}: M \rightarrow M / \mathrm{G}$ be a left principal G-bundle with a $\mathrm{G}$-invariant metric $\mathrm{g}$, and let $\mathcal{G}$ be a gerbe over $M$ with a $\mathrm{G}$-invariant curvature $\mathrm{H}$ admitting a $\mathfrak{g}$ equivariantly closed extension $\widehat{\mathrm{H}}=\mathrm{H}-\kappa$ for $\kappa \in \Omega^{1}(M) \otimes \mathfrak{g}^{*}$. The gerbe $\underline{\mathcal{G}}=\mathcal{G} \otimes I_{\beta}$ defined in terms of the 2-form $\beta$ given in Eq. (9.4) admits a $(\mathrm{G}, 0)$-equivariant structure iff $\mathcal{G}$ is endowed with a $(\mathrm{G}, \rho)$-equivariant structure, with $\rho$ as in Eq. (2.30).

Proof. The claim of the proposition is an immediate consequence of the following observation: The G-invariance of $\beta$, expressed by the identity

$$
{ }^{M} \ell_{g}^{*} \beta=\beta
$$

valid for any $g \in \mathrm{G}$, implies, in virtue of Eqs. (2.34) and (9.10), the identity

$$
\begin{aligned}
{ }^{M} \ell^{*} \beta(g, m) & =\mathrm{e}^{-\overline{\theta_{L}(g)}} \cdot \beta(m)=\beta(m)+\theta_{L}^{a}(g) \wedge \kappa_{a}(m)+\frac{1}{2} \mathrm{c}_{a b}(m) \theta_{L}^{a}(g) \wedge \theta_{L}^{b}(g) \\
& \equiv \beta(m)-\rho(g, m)
\end{aligned}
$$

and hence

$$
{ }^{M} \ell^{*} \underline{\mathcal{G}}={ }^{M} \ell^{*} \mathcal{G} \otimes I_{M_{\ell^{*} \beta}}={ }^{M} \ell^{*} \mathcal{G} \otimes I_{-\rho} \otimes I_{\beta_{2^{*}}} .
$$

Thus, the existence of a $(\mathrm{G}, \rho)$-structure $(\Upsilon, \gamma ; \kappa)$ on $\mathcal{G}$ is tantamount to the existence of the desired $(\mathrm{G}, 0)$-structure $(\underline{\Upsilon}, \underline{\gamma} ; 0)$ on $\underline{\mathcal{G}}$, with $\underline{\Upsilon}:=\Upsilon \otimes \mathrm{id}$ and $\underline{\gamma}:=\gamma \otimes \mathrm{id}$ 
Next, we take a closer look at the effective bi-brane structure determined by the defect condition (9.1) and the formerly established effective target-space geometry. The first result is contained in

Proposition 9.4. Under the assumptions and in the notation of Proposition 9.3, let $\varpi_{Q}$ : $Q \rightarrow Q / \mathrm{G}$ be a left principal G-bundle equipped with a pair of smooth $\mathrm{G}-$ maps $\iota_{\alpha}: Q \rightarrow$ $M, \alpha=1,2$, and with a 2-form $\omega$ which admits a $\mathrm{G}$-equivariant extension $\widehat{\omega}=\omega-k, k \epsilon$ $\Omega^{0}(Q) \otimes \mathfrak{g}^{*}$ satisfying Eq. (3.27). Then, the subset $Q \subset Q$ defined in Eq. (9.5) is G-invariant and, assuming that $\underline{Q}$ is a submanifold of $Q$, the pullback of the 2-form $\underline{\omega}$ of Eq. (9.6) to $\underline{Q}$ is $\mathrm{G}-$ basic.

Proof. The G-invariance of $Q \subset Q$ follows from the G-equivariance of $k$. The G-invariance of $\underline{\omega}$ on $Q$ (a consequence of the same property of $\omega$ and $\beta$ ) then ensures the G-invariance of its restriction (pullback) to $Q$. Finally, we find

$$
\imath_{a} \underline{\omega} \equiv \imath_{a} \omega-\Delta_{Q}\left(\imath_{a} \beta\right)=-\mathrm{d} k_{a},
$$

and so we conclude that $\left.\underline{\omega}\right|_{\underline{Q}}$ is $\mathfrak{g}$-horizontal.

The last proposition enables us to formulate

Proposition 9.5. Let $\varpi_{M}: M \rightarrow M / \mathrm{G}$ be a left principal G-bundle with a $\mathrm{G}$-invariant metric $\mathrm{g}$, and let $(\mathcal{G}, \Upsilon, \gamma ; \kappa)$ be a $(\mathrm{G}, \rho)$-equivariant gerbe over $M$. Furthermore, let $(\underline{\mathcal{G}}, \underline{\Upsilon}, \gamma ; 0)$ be the (G,0)-equivariant gerbe defined in (the proof of) Proposition 9.3 in terms of the 2-form $\beta$ given in Eq.(9.4). Finally, let $\mathcal{B}=\left(Q, \iota_{1}, \iota_{2}, \omega, \Phi\right)$ be a $\mathcal{G}$-bi-brane with a world-volume given by a left principal G-bundle $\varpi_{Q}: Q \rightarrow Q / \mathrm{G}$, equipped with a pair of $\mathrm{G}$-equivariant bi-brane maps $\iota_{\alpha}$, and of a $\mathrm{G}$-invariant curvature $\omega$ which admits a $\mathrm{G}$-equivariant extension $\widehat{\omega}=\omega-k, k \in \Omega^{0}(Q) \otimes \mathfrak{g}^{*}$ satisfying Eq. (3.27). The $(\underline{\mathcal{G}}, \underline{\Upsilon}, \underline{\gamma} ; 0)$-bi-brane $\underline{\mathcal{B}}=\left(\underline{Q}, \iota_{1}, \iota_{2}, \underline{\omega}, \underline{\Phi}\right)$, defined through Eqs. (9.5)-(9.7) and Eq. (9.4), admits a $(\overline{\mathrm{G}}, 0)$-equivariant structure iff the restriction of $\mathcal{B}$ to $\underline{Q}$ is endowed with a $(\overline{\mathrm{G}}, \lambda)$-equivariant structure, with $\lambda$ as in Eq. (2.31) and hence vanishing on $\underline{Q}$.

Proof. First of all, we verify, by recalling the claim of the previous proposition and through direct calculation

$$
\mathrm{d} \underline{\omega} \equiv \mathrm{d} \omega-\Delta_{Q} \mathrm{~d} \beta=-\Delta_{Q}(\mathrm{H}+\mathrm{d} \beta) \equiv-\Delta_{Q} \underline{\mathrm{H}},
$$

that the 2 -form $\underline{\omega}$ has all the properties of the curvature of $\underline{\mathcal{B}}$. We then readily check that the diagram defining the 2-isomorphism $\Xi$ of the $(\mathrm{G}, 0)$-equivariant structure sought after can be obtained from the same diagram for $\Xi$ by tensoring all gerbe entries by $I_{Q_{d_{1}^{(1) *} \iota_{1}^{*} \beta}}$ and all 1-isomorphism entries by the corresponding identity factor. Thus, altogether, the existence of a $(\mathrm{G}, \lambda=0)$-structure on $\mathcal{B}$ over $Q$ is manifestly equivalent to the existence of the desired $(\mathrm{G}, 0)$-structure $(\underline{\Xi} ; 0)$ on $\underline{\mathcal{B}}$, with $\underline{\underline{\Xi}}:=\Xi \otimes \mathrm{id}$.

Finally, we have

Proposition 9.6. Under the assumptions and in the notation of Proposition 9.9, and for $\underline{\mathcal{B}}=\left(\underline{Q}, \iota_{\alpha}, \underline{\omega}, \underline{\Phi}\right)$ the $(\underline{\mathcal{G}}, \underline{\Upsilon}, \underline{\gamma} ; 0)$-bi-brane of that proposition, with data as in Eqs. (9.5)-(9.7), let $\overline{\mathcal{J}}=\left(T_{n},\left(\varepsilon_{n}^{k, k+1}, \pi_{n}^{k}, k+1\right) ; \varphi_{n}\right)$ be a $(\mathcal{G}, \mathcal{B})$-inter-bi-brane with component world-volumes given by left principal $\mathrm{G}$-bundles $\varpi_{T_{n}}: T_{n} \rightarrow T_{n} / \mathrm{G}$, equipped with the respective $\mathrm{G}$-equivariant inter-bi-brane maps $\pi_{n}^{k, k+1}: T_{n} \rightarrow Q$ and such that condition (3.28) is satisfied on $T_{n}$. Then, the $(\underline{\mathcal{G}}, \underline{\mathcal{B}})$-inter-bi-brane $\underline{\mathcal{J}}=\left(\underline{T}_{n},\left(\varepsilon_{n}^{k, k+1}, \pi_{n}^{k, k+1}\right) ; \underline{\varphi}_{n}\right)$, defined through Eqs. (9.8) and (9.9), admits a G-equivariant structure iff the restriction of $\mathcal{J}$ to $\sqcup_{n \geq 3} \underline{T}_{n}$ is endowed with a G-equivariant structure.

Proof. Obvious.

The findings of the present section, in conjunction with Corollary 8.18 , yield the important 
Theorem 9.7. Let $\mathfrak{B}$ be a string background with a target space given by a left principal $\mathrm{G}$-bundle $\mathscr{F} \rightarrow \mathscr{F} / \mathrm{G}$, and assume that $\mathfrak{B}$ is endowed with a $(\mathrm{G}, \rho, \lambda)$-equivariant structure. It then canonically defines a descendendant background $\mathfrak{b}$ with the quotient $\mathscr{F} / G$ as the target space, where the disjoint components of $\underline{\mathscr{F}}=M \sqcup \underline{Q} \sqcup \sqcup_{n \geq 3} \underline{T}_{n}$ are, besides $M$, the spaces defined in Eqs.(9.5) and (9.8), both assumed smooth. Furthermore, it induces a dilaton field on $\mathscr{F} / \mathrm{G}$.

The theorem emphasises the rôle played by a G-equivariant structure on the string background in realizing a $\sigma$-model with the quotient target through the coupling of the original $\sigma$-model to the fluctuating gauge field, with the latter procedure well defined even if the quotient target is singular. As we demonstrate in the sections that follow, a G-equivariant structure on the string background is also sufficient to couple the $\sigma$-model to topologically nontrivial world-sheet gauge fields.

\section{The coupling to arbitrary gauge Fields}

The study (and partial resolution) in Refs. SY90a, SY90b, Hor96, FSS96, GSW10] of the field-identification problem in coset models of CFT seems to indicate that one should put topologically nontrivial world-sheet gauge fields on the same footing as the topologically trivial ones. The reasoning presented in Section 3 suggests an extension of the gauging recipe worked out for gauge fields defined as global Lie algebra $\mathfrak{g}$-valued 1-forms on $\Sigma$ to the situation in which the gauge field represents a connection on a possibly nontrivial principal G-bundle

$$
\pi_{\mathrm{P}}: \mathrm{P} \rightarrow \Sigma .
$$

The bundle comes with the structure of a G-space with the left G-action given by

$$
\mathrm{P}_{\ell}: \mathrm{G} \times \mathrm{P} \rightarrow \mathrm{P}: p \mapsto g \cdot p \equiv r\left(p, g^{-1}\right),
$$

where $r$ is the defining (right) G-action on $\mathrm{P}$. The connections on $\mathrm{P}$ are described by $\mathfrak{g}$-valued 1-forms $\mathcal{A}$ on $\mathrm{P}$ such that

$$
{ }^{\mathrm{P}} \ell_{g}^{*} \mathcal{A}=\operatorname{Ad}_{g} \mathcal{A}
$$

and such that the identity

$$
\imath_{a} \mathcal{A}=t_{a} \quad \text { for } \quad \imath_{a}=\iota_{\mathrm{P}_{\mathscr{K}_{a}}}
$$

holds for the fundamental vector fields ${ }^{\mathrm{P}} \mathscr{K}_{a}$ generating the G-action ${ }^{\mathrm{P}} \ell$. It may then seem natural to replace the world-sheet $\Sigma$ in the definition of the extended target $\Sigma \times \mathscr{F}$ by $\mathrm{P}$ (and its restrictions to subsets of $\Sigma$ ). We shall set

$$
\tilde{\mathscr{F}}:=(\mathrm{P} \times M) \sqcup\left(\left.\mathrm{P}\right|_{\Gamma \backslash \mathfrak{V}_{\Gamma}} \times Q\right) \sqcup \bigsqcup_{n \geq 3}\left(\left.\mathrm{P}\right|_{\mathfrak{V}_{\Gamma}^{(n)}} \times T_{n}\right) \equiv \tilde{M} \sqcup \tilde{Q} \sqcup \bigsqcup_{n \geq 3} \tilde{T}_{n} .
$$

It is for such extended target space that we can define a natural counterpart of the extended string background $\mathfrak{B}_{\mathcal{A}}$ from Corollary 3.17. In close analogy with the discussion of Section 3, and - in particular - with Corollary 3.17, we give

Definition 10.1. Let $\Sigma$ be a world-sheet with an embedded defect quiver $\Gamma$, and let $\pi_{\mathrm{P}}$ : $\mathrm{P} \rightarrow \Sigma$ be a principal G-bundle over $\Sigma$ with connection $\mathcal{A} \in \Omega^{1}(\mathrm{P}) \otimes \mathfrak{g}$. Consider a string background $\mathfrak{B}=(\mathcal{M}, \mathcal{B}, \mathcal{J})$, with target space $\mathscr{F}=M \sqcup Q \sqcup T$, and suppose that $\mathscr{F}$ carries the structure of a G-space, and that the string background $\mathfrak{B}$ is G-equivariant. A P-extension of string background $\mathfrak{B}$, or a P-extended string background, is the string background $\tilde{\mathfrak{B}}_{\mathcal{A}}:=\left(\tilde{\mathcal{M}}_{\mathcal{A}}, \tilde{\mathcal{B}}_{\mathcal{A}}, \tilde{\mathcal{J}}_{\mathcal{A}}\right)$ with the following components

- the P-extended target $\tilde{\mathcal{M}}_{\mathcal{A}}$ composed of the target space $\tilde{M}=\mathrm{P} \times M$ with the metric

$$
\tilde{\mathrm{g}}_{\mathcal{A}}=\mathrm{g}_{2^{*}}-\mathrm{K}_{a 2^{*}} \otimes \mathcal{A}_{1^{*}}^{a}-\mathcal{A}_{1^{*}}^{a} \otimes \mathrm{K}_{a 2^{*}}+\mathrm{h}_{a b 2^{*}}\left(\mathcal{A}^{a} \otimes \mathcal{A}^{b}\right)_{1^{*}}
$$

(defined in terms of the 1-forms $\mathrm{K}_{a}$ of Eq. (3.18) and of the symmetric tensor $\mathrm{h}_{a b}$ of Eq. (3.19)) and the gerbe $\tilde{\mathcal{G}}_{\mathcal{A}}=\mathcal{G}_{2^{*}} \otimes I_{\tilde{\rho}_{\mathcal{A}}}$, with

$$
\tilde{\rho}_{\mathcal{A}}=\kappa_{a 2^{*}} \wedge \mathcal{A}_{1^{*}}^{a}-\frac{1}{2} \mathrm{c}_{a b 2^{*}} \mathcal{A}_{1^{*}}^{a} \wedge \mathcal{A}_{1^{*}}^{b}
$$


cf. Eq. (3.15), of the curvature

$$
\tilde{\mathrm{H}}_{\mathcal{A}}=\mathrm{H}_{2^{*}}+\mathrm{d} \tilde{\rho}_{\mathcal{A}}
$$

cf. Eq. (3.22);

- the P-extended $\tilde{\mathcal{G}}_{\mathcal{A}}$-bi-brane $\tilde{\mathcal{B}}_{\mathcal{A}}$ with the world-volume $\tilde{Q}=\left.\mathrm{P}\right|_{\Gamma} \times Q$, the bi-brane maps $\tilde{\iota}_{\alpha}=\operatorname{id}_{\mathrm{P}} \times \iota_{\alpha}, \alpha=1,2$, the curvature

$$
\tilde{\omega}_{\mathcal{A}}=\omega_{2^{*}}-\Delta_{\tilde{Q}} \tilde{\rho}_{\mathcal{A}}+\mathrm{d} \tilde{\lambda}_{\mathcal{A}}
$$

cf. Eq. (3.29), written in terms of

$$
\tilde{\lambda}_{\mathcal{A}}=-k_{a 2^{*}} \mathcal{A}_{1^{*}}^{a},
$$

cf. Eq. (3.16), and the 1-isomorphism $\tilde{\Phi}_{\mathcal{A}}=\Phi_{2^{*}} \otimes J_{\tilde{\lambda}_{\mathcal{A}}}$;

- the P-extended $\left(\tilde{\mathcal{G}}_{\mathcal{A}}, \tilde{\mathcal{B}}_{\mathcal{A}}\right)$-inter-bi-brane $\tilde{\mathcal{J}}_{\mathcal{A}}$ with component world-volumes $\tilde{T}_{n}=$ $\left.\mathrm{P}\right|_{\mathfrak{V}_{\Gamma}^{(n)}} \times T_{n}, n \geq 3$, with inter-bi-brane maps $\tilde{\pi}_{n}^{k, k+1}=\mathrm{id}_{\mathrm{P}} \times \pi_{n}^{k, k+1}, k=1,2, \ldots, n$, and 2-isomorphisms $\tilde{\varphi}_{n \mathcal{A}}=\varphi_{n 2^{*}}$.

The rationale behind the introduction of the P-extended string background is that it permits to rephrase the gauge coupling in terms of purely geometric constructs in a manner that generalises the treatment of the topologically trivial case. On the other hand, the target space $\tilde{\mathscr{F}}$ of the P-extended string background is not the physical space of the corresponding gauged $\sigma$-model that we strive to define. In order to keep the original field content, we have to pass to the (smooth) quotient $\tilde{\mathscr{F}} / \mathrm{G} \cong \mathscr{F}$ with respect to the combined (left) action

$$
\tilde{\mathscr{F}} \ell: \mathrm{G} \times \tilde{\mathscr{F}} \rightarrow \tilde{\mathscr{F}}:(g,(p, x)) \mapsto\left({ }^{\mathrm{P}} \ell(g, p),{ }^{\mathscr{F}} \ell(g, x)\right),
$$

thereby arriving at associated bundles. This is straightforward on the level of the target space, and the true challenge is to ensure that also the geometric structure supported by $\widehat{\mathscr{F}}$ descends to the quotient space. Here, the results of Section 8.2 will prove instrumental.

10.1. Equivariance properties of P-extended string backgrounds. A convenient language in which to discuss the behaviour of various tensorial and cohomological structures on the P-extended target space $\tilde{\mathscr{F}}$ was introduced in Section 2.2. Here, we merely specialise it to the case of interest. Thus, we consider $\tilde{\mathscr{F}}$ as a G-space equipped with the left action ${ }^{\tilde{\mathscr{F}}} \ell$ defined in Eq. (10.9) and giving rise to the fundamental vector fields

$$
\tilde{\mathscr{F}} \mathscr{K}_{a}(p, x)={ }^{\mathrm{P}} \mathscr{K}_{a}(p)+{ }^{\mathscr{F}} \mathscr{K}_{a}(x), \quad a=1,2, \ldots, \operatorname{dim} \mathfrak{g},
$$

and subsequently construct the nerve G $\tilde{\mathscr{F}}$ of the action groupoid $\mathrm{G} \ltimes \tilde{\mathscr{F}}$, with its face maps $\tilde{\mathscr{F}} d_{i}^{(n+1)}$. Furthermore, the G-equivariant maps $\iota_{\alpha}^{(m)}$ and $\pi_{n}^{k, k+1(m)}$, introduced in Eq. (2.22), admit G-equivariant extensions

$$
\begin{aligned}
\tilde{\iota}_{\alpha}^{(m)} & :=\operatorname{id}_{\mathrm{G}^{m}} \times\left(\operatorname{id}_{\mathrm{P}} \times \iota_{\alpha}\right): \mathrm{G}^{m} \times \tilde{Q} \rightarrow \mathrm{G}^{m} \times \tilde{M}, \\
\tilde{\pi}_{n}^{k, k+1(m)} & :=\operatorname{id}_{\mathrm{G}^{m}} \times\left(\mathrm{id}_{\mathrm{P}}, \pi_{n}^{k, k+1}\right): \mathrm{G}^{m} \times \tilde{T}_{n} \rightarrow \mathrm{G}^{m} \times \tilde{Q} .
\end{aligned}
$$

They provide us with the standard pullback operators $\Delta_{\tilde{Q}}^{(m)}$ and $\Delta_{\tilde{T}_{n}}^{(m)}$, respectively. Together with the pullback-cohomology operators $\tilde{\mathscr{M}}_{\mathrm{G}}^{(m)}$ produced from the face maps of G $\tilde{\mathscr{F}}$, they satisfy the familiar algebra

$$
\begin{gathered}
\tilde{\mathscr{M}}_{\mathrm{G}}^{(m)} \circ \tilde{\mathscr{M}}_{\mathrm{G}}^{(m-1)}=0, \quad \tilde{\mathscr{M}}=\tilde{M}, \tilde{Q}, \tilde{T}_{n}, \\
\tilde{Q}_{\mathrm{G}}^{(m)} \circ \Delta_{\tilde{Q}}^{(m)}=\Delta_{\tilde{Q}}^{(m+1)} \circ \tilde{M} \delta_{\mathrm{G}}^{(m)}, \quad \tilde{T}_{n} \delta_{\mathrm{G}}^{(m)} \circ \Delta_{\tilde{T}_{n}}^{(m)}=\Delta_{\tilde{T}_{n}}^{(m+1)} \circ \tilde{Q}_{\delta_{\mathrm{G}}}^{(m)} .
\end{gathered}
$$

Finally, upon equipping the constituent subspaces of G $\tilde{\mathscr{F}}$ with the structure of a G-space through Eq. (2.19), we obtain the corresponding fundamental vector fields

$$
\tilde{\mathscr{F}}_{\mathscr{K}_{a}^{(m)}}^{(m}\left(g_{m}, g_{m-1}, \ldots, g_{1}, p, x\right)={ }^{\mathrm{P}} \mathscr{K}_{a}(p)+{ }^{\mathscr{F}} \mathscr{K}_{a}^{(m)}\left(g_{m}, g_{m-1}, \ldots, g_{1}, x\right),
$$

in conformity with Eq. (2.20).

In the remainder of this section, we shall study the behaviour of various tensors and the associated geometric objects (gerbes, gerbe bi-modules etc.), involved in the description of 
the $\sigma$-model coupled to topologically nontrivial gauge fields, under the transport within G $\tilde{\mathscr{F}}$ effected by operators $\tilde{\mathscr{M}}_{\mathrm{G}}^{(n)}$ and intertwined by $\Delta_{\tilde{Q}}^{(m)}$ and $\Delta_{\tilde{T}_{n}}^{(m)}$. (We shall systematically add the tilde over all objects that live on the P-extended spaces.) This will pave the way to a subsequent formalisation of our description of the gauged $\sigma$-model in the presence of the defect $\Gamma$.

We begin with

Proposition 10.2. Let $\tilde{\mathcal{M}}_{\mathcal{A}}$ be a P-extended target of Definition 10.1, with a (left) G-action on $\tilde{M}$ given by Eq.(10.9). The metric $\tilde{\mathrm{g}}_{\mathcal{A}}$ on the target space $\tilde{M}$ is $\mathrm{G}$-basic.

Proof. The G-invariance of $\tilde{\mathrm{g}}_{\mathcal{A}}$ is a consequence of the same property of $\mathrm{g}$, as well as of the G-equivariance of the fundamental vector fields ${ }^{M} \mathscr{K}_{a}$. That the extended metric is also $\mathfrak{g}$-horizontal readily follows from Eq. (10.2).

Passing to the differential forms, we establish

Proposition 10.3. GSW10, Lemma 5.4 \& Eq. $(5.13)]$ Let $\tilde{\mathcal{M}}_{\mathcal{A}}=\left(\tilde{M}, \tilde{\mathrm{g}}_{\mathcal{A}}, \tilde{\mathcal{G}}_{\mathcal{A}}\right)$ be a Pextended target of Definition 10.1, with a (left) G-action on $\tilde{M}$ given by Eq. (10.9) and the canonical projections $\mathrm{pr}_{1}: \tilde{M} \rightarrow \mathrm{P}$ and $\mathrm{pr}_{2}: \tilde{M} \rightarrow M$. Moreover, let $\mathrm{G} \tilde{M}$ be the nerve, with face maps $\tilde{M}_{i}^{(m)}$ and the corresponding coboundary operators 2.21), of the action groupoid $\mathrm{G} \ltimes \tilde{M}$ over $\tilde{M}$. Then, the following holds true for $\tilde{\mathrm{H}}_{\mathcal{A}}$ and $\tilde{\rho}_{\mathcal{A}}$ defined in Eq. (10.6) and Eq. (10.5), respectively.

i) $\tilde{\mathrm{H}}_{\mathcal{A}}$ and $\tilde{\rho}_{\mathcal{A}}$ satisfy the relations

$$
\begin{aligned}
{ }^{\tilde{M}} \delta_{\mathrm{G}}^{(0)} \tilde{\rho}_{\mathcal{A}}+\rho_{[1,3]^{*}} & =0, \\
\tilde{M}^{\tilde{G}} \delta_{\mathrm{G}}^{(0)} \tilde{\mathrm{H}}_{\mathcal{A}} & =0, \\
\tilde{M}_{h} \ell_{h}^{*} \tilde{\rho}_{\mathcal{A}} & =\tilde{\rho}_{\mathcal{A}},
\end{aligned}
$$

for $\operatorname{pr}_{1,3}: \mathrm{G} \times \tilde{M} \rightarrow \mathrm{G} \times M$ the canonical projection, and $\rho$ as in Eq. (2.30);

ii) $\tilde{\mathrm{H}}_{\mathcal{A}}$ is $\mathrm{G}$-basic and $\mathfrak{g}$-equivariantly closed.

Proof. Ad i) Identities (10.13) and (10.14) were proven in Ref. GSW10 as Lemma 5.4 and Eq. (5.13), respectively. The former implies identity (10.15) upon pullback along the $\operatorname{map}{ }^{M} \tilde{\iota}_{h}: \tilde{M} \rightarrow \mathrm{G} \times \tilde{M}: \tilde{m} \mapsto(h, \tilde{m})$.

Ad ii) For the sake of the proof, it will be convenient to view $\tilde{\mathrm{H}}_{\mathcal{A}}$ as the Weil transform

$$
\tilde{\mathrm{H}}_{\mathcal{A}}=\mathrm{e}^{-\overline{\mathcal{A}_{1 *}}} \cdot \widehat{\mathrm{H}}_{2^{*}}\left(\left(\pi_{\mathrm{P}}^{*} \mathrm{~F}\right)_{1^{*}}\right)
$$

of $\widehat{\mathrm{H}}$ evaluated on the pullback

$$
\pi_{\mathrm{P}}^{*} \mathrm{~F}:=\mathrm{d} \mathcal{A}+\mathcal{A} \wedge \mathcal{A}
$$

of the curvature $\mathrm{F}$ of $\mathcal{A}$ along (the composition of the canonical projection $\mathrm{pr}_{1}$ with) the bundle projection $\pi_{\mathrm{P}}$, cf. Proposition 3.7. Indeed, upon recalling Eqs. (3.23), we obtain

$$
\begin{aligned}
\mathrm{e}^{-\overline{\mathcal{A}_{1^{*}}}} \cdot \widehat{\mathrm{H}}_{2^{*}}\left(\left(\pi_{\mathrm{P}}^{*} \mathrm{~F}\right)_{1^{*}}\right) \\
=\mathrm{H}_{2^{*}}-\kappa_{a 2^{*}} \wedge\left(\mathrm{d} \mathcal{A}^{a}+\frac{1}{2} f_{a b c} \mathcal{A}^{b} \wedge \mathcal{A}^{c}\right)_{1^{*}}+\mathcal{A}_{1^{*}}^{a} \wedge \mathrm{d} \kappa_{a 2^{*}} \\
\quad+\frac{1}{2}\left(\mathcal{A}^{a} \wedge \mathcal{A}^{b}\right)_{1^{*}}\left(f_{a b c} \kappa_{c}-\mathrm{dc}_{a b}\right)_{2^{*}} \\
\quad-\frac{1}{3 !}\left(\mathcal{A}^{a} \wedge \mathcal{A}^{b} \wedge \mathcal{A}^{c}\right)_{1^{*}}\left(\left(f_{b c d} \imath_{a}+f_{a b d} \imath_{c}-f_{a c d} \imath_{b}\right) \kappa_{d}\right)_{2^{*}} \\
\quad+\mathrm{c}_{a b 2^{*}}\left(\mathcal{A}^{a} \wedge\left(\mathrm{d} \mathcal{A}^{b}+\frac{1}{2} f_{b c d} \mathcal{A}^{c} \wedge \mathcal{A}^{d}\right)\right)_{1^{*}} \\
=\quad \mathrm{H}_{2^{*}}-\kappa_{a 2^{*}} \wedge \mathrm{d} \mathcal{A}_{1^{*}}^{a}+\mathcal{A}_{1^{*}}^{a} \wedge \mathrm{d} \kappa_{a 2^{*}}-\frac{1}{2} \mathrm{~d}\left(\imath_{a} \kappa_{b}\right)_{2^{*}} \wedge\left(\mathcal{A}^{a} \wedge \mathcal{A}^{b}\right)_{1^{*}}
\end{aligned}
$$




$$
+\mathrm{c}_{a b 2^{*}}\left(\mathcal{A}^{a} \wedge \mathrm{d} \mathcal{A}^{b}\right)_{1^{*}}
$$

where the two terms trilinear in $\mathcal{A}$ are readily seen to cancel out. That the above gives the desired result is verified through a direct computation using identity (3.8).

With the help of Eqs. (2.27), (3.6) and (10.2), as well as of the identity

$$
\imath_{a} \mathrm{~d} \mathcal{A}^{b}=f_{a b c} \mathcal{A}^{c}
$$

that follows from the definition of $\mathrm{F}$ and Eq. (10.2), we now establish

$$
\begin{aligned}
\imath_{a} \tilde{\mathrm{H}}_{\mathcal{A}} \\
=\quad\left(\imath_{a} \mathrm{H}\right)_{2^{*}}-\mathrm{c}_{a b 2^{*}} \wedge \mathrm{d} \mathcal{A}_{1^{*}}^{b}+f_{a b c} \kappa_{b 2^{*}} \wedge \mathcal{A}_{1^{*}}^{c}+\mathrm{d} \kappa_{a 2^{*}}+\left(\imath_{a} \mathrm{~d} \kappa_{b}\right)_{2^{*}} \wedge \mathcal{A}_{1^{*}}^{b} \\
\quad-\frac{1}{2}\left(\imath_{a} \mathrm{~d}\left(\imath_{b} \kappa_{c}\right)\right)_{2^{*}}\left(\mathcal{A}^{b} \wedge \mathcal{A}^{c}\right)_{1^{*}}+\mathrm{dc}_{a b 2^{*}} \wedge \mathcal{A}_{1^{*}}^{b}+\mathrm{c}_{a b 2^{*}} \mathrm{~d} \mathcal{A}_{1^{*}}^{b} \\
\quad-f_{a c d}\left(\imath_{b} \kappa_{c}\right)_{2^{*}}\left(\mathcal{A}^{b} \wedge \mathcal{A}^{d}\right)_{1^{*}} \\
=\quad\left(\frac{1}{2}\left(f_{a c d} c_{b d}-f_{a b d} \mathrm{c}_{d c}+\imath_{b} \mathscr{L}_{a} \kappa_{c}\right)\right)_{2^{*}}\left(\mathcal{A}^{b} \wedge \mathcal{A}^{c}\right)_{1^{*}}=0,
\end{aligned}
$$

which shows that $\tilde{\mathrm{H}}_{\mathcal{A}}$ is G-horizontal. Since it is also G-invariant, which can be seen by pulling back Eq. (10.14) along ${ }^{M} \tilde{\iota}_{h}$, it is G-basic, as claimed. Being closed, it is then automatically $\mathfrak{g}$-equivariantly closed.

Passing to the defect data, we find

Proposition 10.4. Under the assumptions and in the notation of Proposition 10.3, let $\tilde{\mathcal{B}}_{\mathcal{A}}=$ $\left(\tilde{Q}, \tilde{\iota}_{\alpha}, \tilde{\omega}_{\mathcal{A}}, \tilde{\Phi}_{\mathcal{A}} \mid \alpha=1,2\right)$ be a P-extended $\tilde{\mathcal{G}}_{\mathcal{A}}$-bi-brane of Definition 10.1, with a (left) Gaction on $\tilde{Q}$ given by Eq. 10.9) and the canonical projections $\operatorname{pr}_{1}: \tilde{Q} \rightarrow \mathrm{P}_{\Gamma}$ and $\operatorname{pr}_{2}: \tilde{Q} \rightarrow Q$. Moreover, let $\mathrm{G} \tilde{Q}$ be the nerve, with face maps $\tilde{Q}_{i}^{(m)}$ and the corresponding coboundary operators (2.21), of the action groupoid $\mathrm{G} \ltimes \tilde{Q}$ over $\tilde{Q}$. Then, the following holds true for $\tilde{\omega}_{\mathcal{A}}$ and $\tilde{\lambda}_{\mathcal{A}}$ defined in Eq. (10.7) and Eq. (10.8), respectively.

i) $\tilde{\omega}_{\mathcal{A}}$ and $\tilde{\lambda}_{\mathcal{A}}$ satisfy the relations

$$
\begin{aligned}
\tilde{Q}_{\mathrm{G}}^{(0)} \tilde{\lambda}_{\mathcal{A}}+\lambda_{[1,3]^{*}} & =0, \\
\tilde{Q}_{\mathrm{G}^{(0)}}^{(0)} \tilde{\omega}_{\mathcal{A}} & =0, \\
\tilde{Q}_{\ell_{g}^{*}} \tilde{\lambda}_{\mathcal{A}} & =\tilde{\lambda}_{\mathcal{A}},,
\end{aligned}
$$

for $\operatorname{pr}_{1,3}: \mathrm{G} \times \tilde{Q} \rightarrow \mathrm{G} \times Q$ the canonical projection, and $\lambda$ as in $E q$. (2.31);

ii) $\tilde{\omega}_{\mathcal{A}}$ is $\mathrm{G}$-basic and it verifies

$$
\widehat{\mathrm{d}} \tilde{\omega}_{\mathcal{A}}=-\Delta_{\tilde{Q}} \tilde{\mathrm{H}}_{\mathcal{A}}
$$

Proof. Ad i) Identity (10.17) follows from Eqs. (2.34), (10.1) and (10.2), upon taking into account the G-equivariance of $k$. It yields identity (10.19) through pullback along the map ${ }^{{ }^{2} \tilde{\iota}_{h}}: \tilde{Q} \rightarrow \mathrm{G} \times \tilde{Q}: \tilde{q} \mapsto(h, \tilde{q})$. Finally, in conjunction with Eqs. (2.41) and (10.13), it gives identity (10.18).

Ad ii) Pulling back Eq. (10.18) along ${ }^{Q_{\iota_{h}}}$, we establish the G-invariance of $\tilde{\omega}_{\mathcal{A}}$. From the simple identities

$$
\imath_{a} \tilde{\rho}_{\mathcal{A}}=-\kappa_{a 2^{*}}, \quad \imath_{a} \mathrm{~d} \tilde{\lambda}_{\mathcal{A}}=\mathrm{d} k_{a 2^{*}},
$$

taken together with Eq. 2.28), we infer that $\tilde{\omega}_{\mathcal{A}}$ is also $\mathfrak{g}$-horizontal,

$$
\imath_{a} \tilde{\omega}_{\mathcal{A}}=0,
$$

so that, altogether, it is G-basic as claimed. Using the last identity, we then show

$$
\widehat{\mathrm{d}} \tilde{\omega}_{\mathcal{A}}\left(t_{a}\right) \equiv \mathrm{d} \tilde{\omega}_{\mathcal{A}}-\imath_{a} \tilde{\omega}_{\mathcal{A}}=(\mathrm{d} \omega)_{2^{*}}-\Delta_{\tilde{Q}} \mathrm{~d} \tilde{\rho}_{\mathcal{A}}=-\Delta_{\tilde{Q}} \tilde{\mathrm{H}}_{\mathcal{A}}\left(t_{a}\right)
$$


whence also Eq. (10.21).

Remark 10.5. We may interpret $\tilde{\omega}_{\mathcal{A}}$ as the Weil transform

$$
\tilde{\omega}_{\mathcal{A}}=\mathrm{e}^{-\overline{\mathcal{A}}_{1^{*}}} \cdot \widehat{\omega}_{2^{*}}\left(\left(\pi_{\mathrm{P}}^{*} \mathrm{~F}\right)_{1^{*}}\right),
$$

cf. Proposition 3.14. Indeed, proceeding along the same lines as in the proof of Eq. (10.16) and invoking Eqs. (2.28) and (3.7) along the way, we find

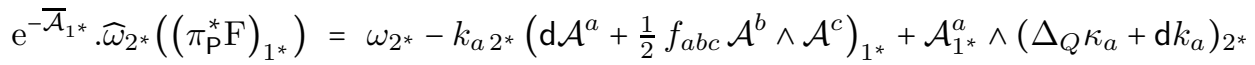

$$
\begin{aligned}
& +\frac{1}{2}\left(\mathcal{A}^{a} \wedge \mathcal{A}^{b}\right)_{1^{*}}\left(\imath_{a}\left(\Delta_{Q} \kappa_{b}+\mathrm{d} k_{b}\right)\right)_{2^{*}} \\
& =\omega_{2^{*}}-k_{a 2^{*}} \mathrm{~d} \mathcal{A}_{1^{*}}^{a}-\mathrm{d} k_{a 2^{*}} \wedge \mathcal{A}_{1^{*}}^{a}-\Delta_{\tilde{Q}}\left(\kappa_{a 2^{*}} \wedge \mathcal{A}_{1^{*}}^{a}\right)+\frac{1}{2} \Delta_{\tilde{Q}}\left(\left(\imath_{a} \kappa_{b}\right)_{2^{*}}\left(\mathcal{A}^{a} \wedge \mathcal{A}^{b}\right)_{1^{*}}\right) .
\end{aligned}
$$

We are now ready to discuss the global geometric structures associated with the forms considered above. Thus, we have

Proposition 10.6. GSW10, Prop. 5.5] Let $(\mathcal{G}, \Upsilon, \gamma ; \kappa)$ be a $(\mathrm{G}, \rho)$-equivariant gerbe over a $\mathrm{G}$-space $M$, and let $\pi_{\mathrm{P}}: \mathrm{P} \rightarrow \Sigma$ be a principal $\mathrm{G}$-bundle with connection $\mathcal{A} \in \Omega^{1}(\mathrm{P}) \otimes \mathfrak{g}$ over an oriented two-dimensional surface $\Sigma$. Denote by $\tilde{M} \equiv \mathrm{P} \times M$ the $\mathrm{P}$-extension of $M$. The quadruple $\left.\left(\tilde{\mathcal{G}}_{\mathcal{A}}, \tilde{\Upsilon}_{\mathcal{A}}, \tilde{\gamma}_{\mathcal{A}} ; 0\right):=\left(\mathcal{G}_{2^{*}} \otimes I_{\tilde{\rho}_{\mathcal{A}}}, \Upsilon_{[1,3]^{*}} \otimes \mathrm{id}, \gamma_{[1,2,4}\right]^{*} \otimes \mathrm{id} ; 0\right)$, written in the notation of Proposition 10.3 and in terms of the canonical projections $\operatorname{pr}_{1}: \tilde{M} \rightarrow \mathrm{P}, \operatorname{pr}_{2}: \tilde{M} \rightarrow M$, $\operatorname{pr}_{1,3}: \mathrm{G} \times \tilde{M} \rightarrow \mathrm{G} \times M$ and $\mathrm{pr}_{1,2,4}: \mathrm{G}^{2} \times \tilde{M} \rightarrow \mathrm{G}^{2} \times M$, carries a canonical structure of a (G,0)-equivariant gerbe over $\tilde{M}$.

Proposition 10.6 points towards a relation between a general G-equivariant structure over a given manifold and a distinguished one over its principal G-extension. This observation leads us immediately to the counterpart of the above that pertains to bi-branes.

Proposition 10.7. Under the assumptions and in the notation of Proposition 10.6, let $Q$ be a G-space equipped with a pair of smooth $\mathrm{G}$-equivariant maps $\iota_{\alpha}: Q \rightarrow M, \alpha=1,2$, and denote by $\left.\tilde{Q} \equiv \mathrm{P}\right|_{\Gamma} \times Q$ its $\mathrm{P}$-extension, for $\Gamma$ a defect quiver without junctions embedded in $\Sigma$. Moreover, let $\left(\tilde{\mathcal{G}}_{\mathcal{A}}, \tilde{\Upsilon}_{\mathcal{A}}, \tilde{\gamma}_{\mathcal{A}} ; 0\right)$ be the $(\mathrm{G}, 0)$-equivariant gerbe over $\tilde{M}$ associated to a $(\mathrm{G}, \rho)$-equivariant gerbe $(\mathcal{G}, \Upsilon, \gamma ; \kappa)$ over $M$. Finally, let $(\mathcal{B}, \Xi ; k)$, with $\mathcal{B}=\left(Q, \iota_{1}, \iota_{2}, \omega, \Phi\right)$, be a $(\mathrm{G}, \lambda)$-equivariant $(\mathcal{G}, \Upsilon, \gamma ; \kappa)$-bi-brane and denote by $\tilde{\Phi}_{\mathcal{A}}$ the 1-isomorphism

$$
\tilde{\Phi}_{\mathcal{A}}=\Phi_{2^{*}} \otimes J_{\tilde{\lambda}_{\mathcal{A}}}: \tilde{\iota}_{1}^{*} \tilde{\mathcal{G}}_{\mathcal{A}} \stackrel{\cong}{\longrightarrow} \tilde{\iota}_{2}^{*} \tilde{\mathcal{G}}_{\mathcal{A}} \otimes I_{\tilde{\omega}_{\mathcal{A}}}
$$

of gerbes over $\tilde{Q}$, written in terms of the smooth maps $\tilde{\iota}_{\alpha} \equiv \tilde{\iota}_{\alpha}^{(0)}$ and the canonical projection $\operatorname{pr}_{2}: \tilde{Q} \rightarrow Q$, as well as of the trivial 1-isomorphism $J_{\tilde{\lambda}_{\mathcal{A}}}$ defined by the 1-form $\tilde{\lambda}_{\mathcal{A}}$ on $\tilde{Q}$ given in Eq. (10.8), and of the 2-form $\tilde{\omega}_{\mathcal{A}}$ on $\tilde{Q}$ defined in Eq. (10.7). Then, there exists a canonical structure of a $(\mathrm{G}, 0)$-equivariant $\left(\tilde{\mathcal{G}}_{\mathcal{A}}, \tilde{\Upsilon}_{\mathcal{A}}, \tilde{\gamma}_{\mathcal{A}} ; 0\right)$-bi-brane on the triple $\left(\tilde{\mathcal{B}}_{\mathcal{A}}, \tilde{\Xi}_{\mathcal{A}} ; 0\right)$ consisting of the bi-brane $\tilde{\mathcal{B}}_{\mathcal{A}}=\left(\tilde{Q}, \tilde{\iota}_{\alpha}, \tilde{\omega}_{\mathcal{A}}, \tilde{\Phi}_{\mathcal{A}}\right)$ over $\tilde{Q}$ and of the 2-isomorphism $\tilde{\Xi}_{\mathcal{A}}=\Xi_{[1,3]^{*}} \otimes \mathrm{id}$.

Proof. First of all, we have to demonstrate that $\tilde{\Phi}_{\mathcal{A}}$ does, indeed, define a $\left(\tilde{\iota}_{1}^{*} \tilde{\mathcal{G}}_{\mathcal{A}}, \tilde{\iota}_{2}^{*} \tilde{\mathcal{G}}_{\mathcal{A}}\right)$-bimodule of curvature $\tilde{\omega}_{\mathcal{A}}$. That it yields the desired 1-isomorphism follows trivially from the very definition of $\tilde{\omega}_{\mathcal{A}}$ as

$$
\begin{aligned}
\tilde{\iota}_{1}^{*} \tilde{\mathcal{G}}_{\mathcal{A}} \equiv \operatorname{pr}_{2}^{*} \iota_{1}^{*} \mathcal{G} \otimes I_{\tilde{\iota}_{1}^{*} \tilde{\rho}_{\mathcal{A}}} \stackrel{\operatorname{pr}_{2}^{*} \Phi \otimes J_{\tilde{\lambda}_{\mathcal{A}}}}{\longrightarrow} & \operatorname{pr}_{2}^{*}\left(\iota_{2}^{*} \mathcal{G} \otimes I_{\omega}\right) \otimes I_{\tilde{L}_{1}^{*} \tilde{\rho}_{\mathcal{A}}+\mathrm{d} \tilde{\lambda}_{\mathcal{A}}} \\
& =\operatorname{pr}_{2}^{*} \iota_{2}^{*} \mathcal{G} \otimes I_{\tilde{L}_{2}^{*} \tilde{\rho}_{\mathcal{A}}} \otimes I_{\omega_{2^{*}}-\Delta_{\tilde{Q}}^{*} \tilde{\rho}_{\mathcal{A}}+\mathrm{d} \tilde{\lambda}_{\mathcal{A}}} \\
& \equiv \tilde{\iota}_{2}^{*} \tilde{\mathcal{G}}_{\mathcal{A}} \otimes I_{\tilde{\omega}_{\mathcal{A}}} .
\end{aligned}
$$

Eq. (10.21) and the G-equivariance of $\tilde{\omega}_{\mathcal{A}}$ ensure that the trivial G-equivariant (Cartanmodel) extension of $\tilde{\omega}_{\mathcal{A}}$ has the properties required of a curvature of the $\left(\tilde{\iota}_{1}^{*} \tilde{\mathcal{G}}_{\mathcal{A}}, \tilde{\iota}_{2}^{*} \tilde{\mathcal{G}}_{\mathcal{A}}\right)$-bimodule which is G-equivariant relative to the vanishing 1-form, as stated in Definition 8.4. 
In the next step, we check that $\tilde{\Xi}_{\mathcal{A}}$ renders the appropriate 2-diagram (8.7) commutative. To this end, we write out the objects appearing in the diagram in the case in hand using the respective definitions given in the proposition, and subsequently apply the (commutation) relations for various projections, bi-brane and face maps involved, and use Eq. (10.17),

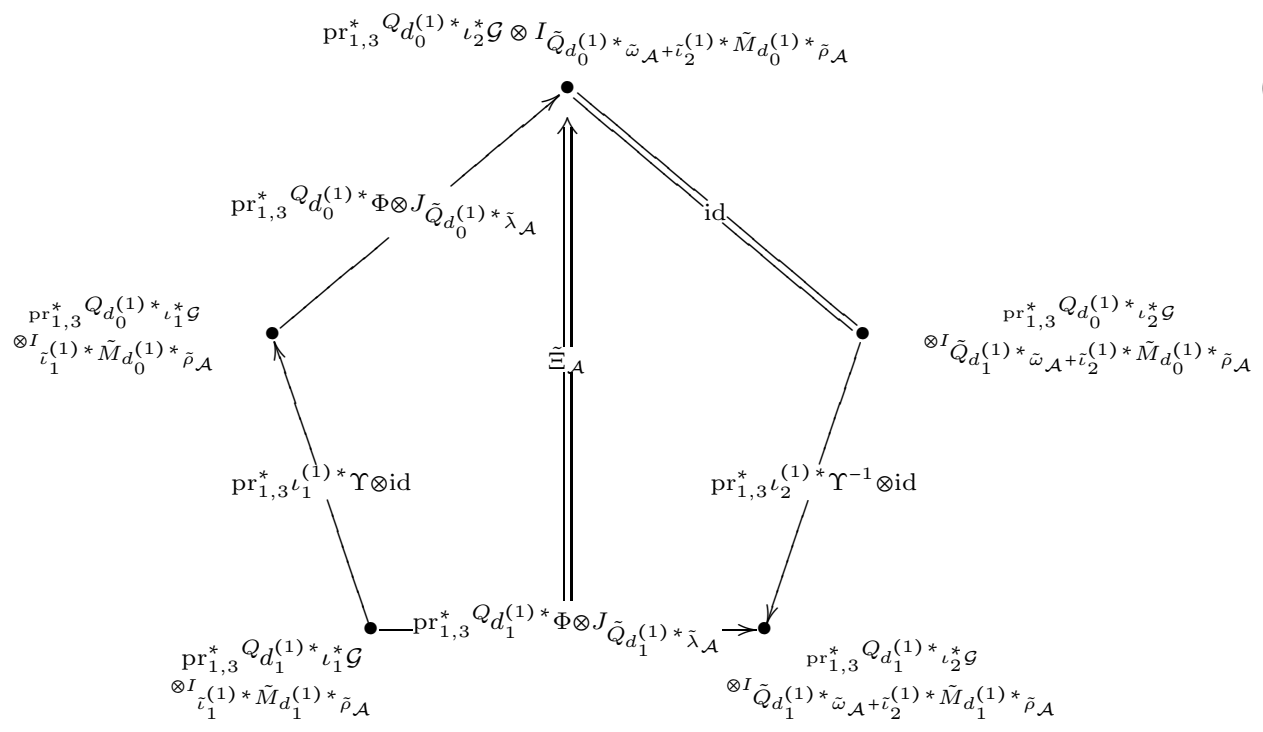

whereupon it becomes clear, in virtue of the assumed properties of $(\mathcal{B}, \Xi ; k)$, that the 2isomorphism $\tilde{\Xi}_{\mathcal{A}}=\operatorname{pr}_{1,3}^{*} \Xi \otimes \mathrm{id}$ renders the diagram meaningful. Having carried out an analogous exercise for diagram (H.4) in the present setting, we conclude that all the 2isomorphisms on the faces of the trigonal prism factor as tensor products of an identity 2-isomorphism with pullbacks, along the canonical projection $\operatorname{pr}_{1,2,4}: \mathrm{G}^{2} \times \tilde{Q} \rightarrow \mathrm{G}^{2} \times Q$, of the 2-isomorphisms from the respective faces of the trigonal prism of the similar diagram for the assumed G-equivariant $\mathcal{G}$-bi-brane structure on $(\mathcal{B}, \Xi ; k)$, and so the 2-isomorphism $\tilde{\Xi}_{\mathcal{A}}$ satisfies the necessary coherence condition of Definition 8.4. This completes the proof of the proposition.

We complete our discussion with a statement concerning an inter-bi-brane.

Proposition 10.8. Under the assumptions and in the notation of Propositions 10.0 and 10.7, let $T_{n}, n \geq 3$, be a collection of $\mathrm{G}$-spaces, equipped with smooth $\mathrm{G}$-equivariant maps $\pi_{n}^{k, k+1}: T_{n} \rightarrow Q, k=1,2, \ldots, n$, and denote by $\left.\tilde{T}_{n} \equiv \mathrm{P}\right|_{\mathfrak{V}_{\Gamma}^{(n)}} \times T_{n}$ the respective $\mathrm{P}$-extensions, for $\mathfrak{V}_{\Gamma}^{(n)}$ the sets of junctions of valence $n$ of the defect quiver $\Gamma \subset \Sigma$. Furthermore, let $\left(\tilde{\mathcal{G}}_{\mathcal{A}}, \tilde{\Upsilon}_{\mathcal{A}}, \tilde{\gamma}_{\mathcal{A}} ; 0\right)$ be $(\mathrm{G}, 0)$-equivariant gerbe over $\tilde{M}$ associated to a $(\mathrm{G}, \rho)$-equivariant gerbe $(\mathcal{G}, \Upsilon, \gamma ; \kappa)$ over $M$, and let $\left(\tilde{\mathcal{B}}_{\mathcal{A}}, \tilde{\Xi}_{\mathcal{A}} ; 0\right)$ be the $(\mathrm{G}, 0)$-equivariant $\left(\tilde{\mathcal{G}}_{\mathcal{A}}, \tilde{\Upsilon}_{\mathcal{A}}, \tilde{\gamma}_{\mathcal{A}} ; 0\right)$-bi-brane over $\tilde{Q}$ associated to a $(\mathrm{G}, \lambda)$-equivariant $(\mathcal{G}, \Upsilon, \gamma ; \kappa)$-bi-brane $(\mathcal{B}, \Xi ; k)$. Finally, let $\mathcal{J}=$ $\left(T_{n},\left(\varepsilon_{n}^{k, k+1}, \pi_{n}^{k, k+1}\right) ; \varphi_{n}\right)$ be a G-equivariant $((\mathcal{G}, \Upsilon, \gamma ; \kappa),(\mathcal{B}, \Xi ; k))$-inter-bi-brane, and denote by $\varphi_{n \mathcal{A}}$ the 2-isomorphisms

$$
\tilde{\varphi}_{n \mathcal{A}}:=\varphi_{n 2^{*}}
$$

between 1-isomorphisms of gerbes over the respective $\tilde{T}_{n}$, with $\operatorname{pr}_{2}: \tilde{T}_{n} \rightarrow T_{n}$ the canonical projections. Then, there exists a canonical structure of a $\mathrm{G}$-equivariant $\left(\left(\tilde{\mathcal{G}}_{\mathcal{A}}, \tilde{\Upsilon}_{\mathcal{A}}, \tilde{\gamma}_{\mathcal{A}} ; 0\right),\left(\tilde{\mathcal{B}}_{\mathcal{A}}\right.\right.$, $\left.\left.\tilde{\Xi}_{\mathcal{A}} ; 0\right)\right)$-inter-bi-brane on $\tilde{\mathcal{J}}_{\mathcal{A}}=\left(\tilde{T}_{n},\left(\tilde{\varepsilon}_{n}^{k, k+1}, \tilde{\pi}_{n}^{k, k+1}\right) ; \tilde{\varphi}_{n \mathcal{A}}\right)$, the latter being defined in terms of the maps $\tilde{\varepsilon}_{n}^{k, k+1}:=\varepsilon_{n}^{k, k+1} \circ \mathrm{pr}_{2}$ and $\tilde{\pi}_{n}^{k, k+1} \equiv \tilde{\pi}_{n}^{k, k+1(0)}$.

Proof. The claim of the proposition is a direct consequence of the chain of equalities

$$
\begin{array}{r}
\left(\tilde{\pi}_{n}^{n, 1 *} \Phi^{\tilde{\varepsilon}_{n}^{n, 1}} \otimes \mathrm{id}\right) \circ\left(\tilde{\pi}_{n}^{n-1, n *} \Phi^{\tilde{\varepsilon}_{n}^{n-1, n}} \otimes \mathrm{id}\right) \circ \cdots \circ \tilde{\pi}_{n}^{1,2 *} \Phi^{\tilde{\varepsilon}_{n}^{1,2}} \\
=\operatorname{pr}_{2}^{*}\left(\left(\Phi_{n}^{n, 1} \otimes \mathrm{id}\right) \circ\left(\Phi_{n}^{n-1, n} \otimes \mathrm{id}\right) \circ \cdots \circ \Phi_{n}^{1,2}\right) \otimes J_{\Delta_{\tilde{T}_{n}}} \tilde{\lambda}_{\mathcal{A}} \\
=\operatorname{pr}_{2}^{*}\left(\left(\Phi_{n}^{n, 1} \otimes \mathrm{id}\right) \circ\left(\Phi_{n}^{n-1, n} \otimes \mathrm{id}\right) \circ \cdots \circ \Phi_{n}^{1,2}\right),
\end{array}
$$


inferred by Eq. (2.29).

We shall now apply the propositions collected in the present section, in conjunction with Theorems 8.10, 8.15 and 8.17 (as summarised in Corollary 8.18) to the P-extended target space $\tilde{\mathscr{F}}$ of Eq. (10.3), equipped with the G-action of Eq. 10.9). The latter turns $\varpi_{\tilde{M}}: \tilde{M} \rightarrow$ $\tilde{M} / \mathrm{G}, \varpi_{\tilde{Q}}: \tilde{Q} \rightarrow \tilde{Q} / \mathrm{G}$ and $\varpi_{\tilde{T}_{n}}: \tilde{T}_{n} \rightarrow \tilde{T}_{n} / \mathrm{G}$ into left principal G-bundles. For these, we obtain the fundamental

Corollary 10.9. Under the assumptions and in the notation of Definition 10.1 and Propositions 10.6, 10.7 and 10.8, the P-extension $\tilde{\mathfrak{B}}_{\mathcal{A}}$ of a $(\mathrm{G}, \rho, \lambda)$-equivariant string background with the P-extended target space $\tilde{\mathscr{F}}=\tilde{M} \sqcup \tilde{Q} \sqcup \sqcup_{n \geq 3} \tilde{T}_{n}$, descends to a unique string background $\mathfrak{B}_{\mathcal{A}}$ with target space $\tilde{\mathscr{F}} / \mathrm{G}$, and the pullbacks of the gerbe $\mathcal{G}_{\mathcal{A}}$, of the bi-brane 1-isomorphism $\Phi_{\mathcal{A}}$ and of the inter-bi-brane 2-isomorphisms $\varphi_{n \mathcal{A}}$ of $\mathfrak{B}_{\mathcal{A}}$ along the respective smooth maps $\varpi_{\tilde{\mathscr{M}}}$ are $\mathrm{G}$-equivariantly equivalent to the $(\mathrm{G}, 0)$-equivariant gerbe, the $(\mathrm{G}, 0)$-equivariant bi-brane 1-isomorphism and the G-equivariant inter-bi-brane 2-isomorphism of $\tilde{\mathfrak{B}}_{\mathcal{A}}$, respectively, in the sense of Definitions 8.9, 8.4 and 8.9. The quotient target space $\tilde{M} / \mathrm{G}$ is equipped with the unique metric $\mathrm{g}_{\mathcal{A}}$ determined by the condition that its pullback to $\tilde{M}$ along $\varpi_{\tilde{M}}$ give $\tilde{\mathrm{g}}_{\mathcal{A}}$.

10.2. Fully gauged $\sigma$-models and their gauge invariance. Network-field configurations $(\psi \mid \Gamma)$ over an oriented Riemann surface $\Sigma$ with an embedded defect quiver $\Gamma$ in the $\sigma$ model coupled to a connection $\mathcal{A}$ on a principal G-bundle $\mathrm{P} \rightarrow \Sigma$ are, by definition (and in accord with the discussion from the opening paragraphs), sections of the disjoint union

$$
\tilde{\mathscr{F}} / \mathrm{G}=(\tilde{M} / \mathrm{G}) \sqcup(\tilde{Q} / \mathrm{G}) \sqcup \bigsqcup_{n \geq 3}\left(\tilde{T}_{n} / \mathrm{G}\right) \cong \mathscr{F}
$$

of the associated bundles $\tilde{M} / \mathrm{G} \equiv \mathrm{P} \times_{\mathrm{G}} M \rightarrow \Sigma, \tilde{Q} /\left.\mathrm{G} \equiv \mathrm{P}\right|_{\Gamma} \times_{\mathrm{G}} Q \rightarrow \Gamma$ and $\tilde{T}_{n} /\left.\mathrm{G} \equiv \mathrm{P}\right|_{\mathfrak{V}_{\Gamma}^{(n)}} \times_{\mathrm{G}}$ $T_{n} \rightarrow \mathfrak{V}_{\Gamma}^{(n)}$. The foregoing analyses motivate

Definition 10.10. Under the assumptions and in the notation of Definition 10.1 and Corollary 10.9, let $(\psi \mid \Gamma)$, with $\psi$ a section of the associated bundles $\tilde{\mathscr{F}} / \mathrm{G}$ of Eq. (10.25), be a network-field configuration over a closed oriented (euclidean) world-sheet $(\Sigma, \gamma)$ with an embedded defect quiver $\Gamma$, in the string background $\mathfrak{B}_{\mathcal{A}}$ descended from a $(\mathrm{G}, 0,0)$-equivariant P-extended string background $\tilde{\mathfrak{B}}_{\mathcal{A}}$ and consisting, in particular, of a metric target space $\left(\tilde{M} / \mathrm{G}, \mathrm{g}_{\mathcal{A}}\right)$ with gerbe $\mathcal{G}_{\mathcal{A}}$ over it, of a $\mathcal{G}_{\mathcal{A}}$-bi-brane $\mathcal{B}_{\mathcal{A}}$ with a gerbe 1 -isomorphism $\Phi_{\mathcal{A}}$, and of a $\left(\mathcal{G}_{\mathcal{A}}, \mathcal{B}_{\mathcal{A}}\right)$-inter-bi-brane $\mathcal{J}_{\mathcal{A}}$ with component gerbe 2 -isomorphisms $\varphi_{n} \mathcal{A}$. The euclidean Feynman amplitude of $(\psi \mid \Gamma)$ is

$$
\mathscr{A}[(\psi \mid \Gamma) ; \gamma, \mathcal{A}]=\exp \left[-\frac{1}{2} \int_{\Sigma} \mathrm{g}_{\mathcal{A}}\left(\mathrm{d} \psi \wedge{ }^{\star_{\gamma}} \mathrm{d} \psi\right)\right] \operatorname{Hol}_{\mathcal{G}_{\mathcal{A}}, \Phi_{\mathcal{A}}, \varphi_{n \mathcal{A}}}(\psi \mid \Gamma) .
$$

In order to introduce gauge transformations in the present context, we should recall the notion of the adjoint bundle $\mathrm{P} \times{ }_{\mathrm{AdG}} \mathrm{G} \rightarrow \Sigma$. Points in the total space $\mathrm{P} \times{ }_{\mathrm{AdG}} \mathrm{G}$ are classes $[(p, g)]$ of the equivalence relation

$$
\left(p \cdot h^{-1}, \operatorname{Ad}_{h}(g)\right) \sim(p, g), \quad h \in \mathrm{G}
$$

on $\mathrm{P} \times \mathrm{G}$, and so they admit point-wise multiplication

$$
\left[\left(p, g_{1}\right)\right] \cdot\left[\left(p, g_{2}\right)\right]:=\left[\left(p, g_{1} \cdot g_{2}\right)\right]
$$

which endows $\mathrm{P} \times{ }_{\mathrm{AdG}} \mathrm{G}$ with the structure of a bundle of groups. The fibre-wise (left) action of $\mathrm{P} \times{ }_{\mathrm{AdG}} \mathrm{G}$ on $\mathrm{P}$

$$
\left(\mathrm{P} \times{ }_{\mathrm{AdG}} \mathrm{G}\right) \times \mathrm{P} \rightarrow \mathrm{P}:\left(\left[\left(p, g_{1}\right)\right], p \cdot g_{2}\right) \mapsto p \cdot\left(g_{1} \cdot g_{2}\right)
$$

induces an action

$$
\lambda: \Gamma\left(\mathrm{P} \times{ }_{\mathrm{AdG}} \mathrm{G}\right) \times \mathrm{P} \rightarrow \mathrm{P}:(\chi, p) \mapsto \lambda_{\chi}(p)
$$

of a section $\chi$ of the adjoint bundle $\mathrm{P} \times{ }_{\mathrm{AdG}} \mathrm{G}$ by an automorphism of $\mathrm{P}$. Note that

$$
\lambda_{\chi} \circ \lambda_{\chi^{\prime}}=\lambda_{\chi \cdot \chi^{\prime}} .
$$


The automorphisms $\lambda_{\chi}$ induce fibre maps ${ }^{\mathscr{M}} L_{\chi}: \mathrm{P} \times_{\mathrm{G}} \mathscr{M} \rightarrow \mathrm{P} \times_{\mathrm{G}} \mathscr{M}$ that descend from the G-equivariant (with respect to the action (10.9)) maps

$$
{ }^{\mathscr{M}} \tilde{L}_{\chi}=\left(\lambda_{\chi}, \mathrm{id} \mathscr{M}\right): \mathrm{P} \times \mathscr{M} \rightarrow \mathrm{P} \times \mathscr{M} .
$$

We have

Definition 10.11. In a field theory on a space-time $\mathscr{S}$ with fields given by sections of a bundle $\mathscr{M} \hookrightarrow \mathrm{P} \times_{\mathrm{G}} \mathscr{M} \rightarrow \mathscr{S}$ associated to the principal G-bundle $\mathrm{P} \rightarrow \mathscr{S}$, and for $\mathscr{M}$ a $\mathrm{G}$ space with a (left) G-action given by Eq. (2.10), the group $\mathrm{G}_{\mathrm{P}}$ of gauge transformations is the set of sections $\Gamma\left(\mathrm{P} \times{ }_{\mathrm{AdG}} \mathrm{G}\right)$ of the adjoint bundle $\mathrm{P} \times{ }_{\mathrm{AdG}} \mathrm{G} \rightarrow \mathscr{S}$, with the group operation induced by the point-wise multiplication of Eq. (10.26). A gauge transformation of the field $\psi \in \Gamma\left(\mathrm{P} \times_{\mathrm{G}} \mathscr{M}\right)$ by $\chi \in \mathrm{G}_{\mathrm{P}}$ is given by

$$
(\chi, \psi) \mapsto{ }^{\mathscr{M}} L_{\chi} \circ \psi=:{ }^{\chi} \psi
$$

where ${ }^{\mathscr{M}} L_{\chi}$ is the unique mapping induced by the G-equivariant map ${ }^{\mathscr{M}} \tilde{L}_{\chi}$ of Eq. (10.28). The associated gauge transformation of the connection $\mathcal{A}$ on $\mathrm{P}$ reads

$$
\mathcal{A} \mapsto \lambda_{\chi^{-1}}^{*} \mathcal{A}=:{ }^{\chi} \mathcal{A}
$$

where $\chi^{-1}$ is to be understood as the point-wise group inverse of $\chi$.

We are finally ready to present the fundamental result of the paper.

Theorem 10.12. The euclidean Feynman amplitude of the gauged $\sigma$-model from Definition 10.10 is invariant under arbitrary gauge transformations, that is, for all $\chi \in \mathrm{G}_{\mathrm{P}}$, the identity

$$
\mathscr{A}\left[\left({ }^{\chi} \psi \mid \Gamma\right) ; \gamma,{ }^{\chi} \mathcal{A}\right]=\mathscr{A}[(\psi \mid \Gamma) ; \gamma, \mathcal{A}]
$$

holds true.

Proof. The gauge invariance of the 'metric' term follows immediately from the relation

$$
{ }^{M} L_{\chi}^{*} \tilde{\mathrm{g}}_{\chi \mathcal{A}}=\tilde{\mathrm{g}}_{\mathcal{A}}
$$

cf. Eq. (10.4). Thus, we are left with the task of proving the same property of the 'topological' term, which is tantamount to verifying the identity

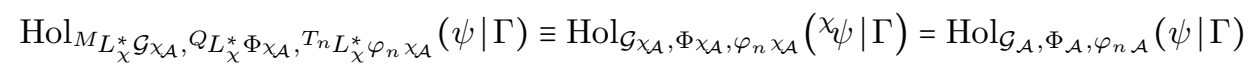

for arbitrary $\chi, \psi$ and $\mathcal{A}$ from the respective domains of definition. The proof extends that of the gauge invariance of the 'topological' term for $\Gamma=\varnothing$, given in Ref. GSW10. Thus, we start by showing the existence of a 1-isomorphism

$$
\Psi_{\mathcal{A}, \chi}:{ }^{M} L_{\chi}^{*} \mathcal{G}_{\chi_{\mathcal{A}}} \stackrel{\cong}{\longrightarrow} \mathcal{G}_{\mathcal{A}}
$$

inferred, via Theorem 8.10, and owing to the G-equivariance of ${ }^{M} \tilde{L}_{\chi}$, from the existence of the corresponding 1-cell in $\mathfrak{B} \mathfrak{G} \mathfrak{r b} \nabla(\tilde{M})_{0}^{\mathrm{G}}$,

$$
\left(\tilde{\Psi}_{\mathcal{A}, \chi}, \tilde{Z}_{\mathcal{A}, \chi}\right):\left({ }^{M} \tilde{L}_{\chi}^{*} \tilde{\mathcal{G}}_{\chi \mathcal{A}},\left(\operatorname{id}_{\mathrm{G}} \times{ }^{M} \tilde{L}_{\chi}\right){ }^{*} \tilde{\Upsilon}_{\chi_{\mathcal{A}}},\left(\operatorname{id}_{\mathrm{G}^{2}} \times{ }^{M} \tilde{L}_{\chi}\right)^{*} \tilde{\gamma}_{\chi \mathcal{A}}\right) \stackrel{\cong}{\longrightarrow}\left(\tilde{\mathcal{G}}_{\mathcal{A}}, \tilde{\Upsilon}_{\mathcal{A}}, \tilde{\gamma}_{\mathcal{A}}\right)
$$

Upon writing out the components of the transformed gerbe

$$
\begin{aligned}
& { }^{M} \tilde{L}_{\chi}^{*} \tilde{\mathcal{G}}_{\chi \mathcal{A}} \equiv{ }^{M} \tilde{L}_{\chi}^{*}\left(\mathcal{G}_{2^{*}} \otimes I_{\tilde{\rho}_{\mathcal{A}}}\right)=\mathcal{G}_{2^{*}} \otimes I_{M} \tilde{L}_{\chi}^{*} \tilde{\rho}_{\chi \mathcal{A}}=\mathcal{G}_{2^{*}} \otimes I_{\tilde{\rho}_{\lambda_{\chi}^{*} \chi \mathcal{A}}}=\mathcal{G}_{2^{*}} \otimes I_{\tilde{\rho}_{\mathcal{A}}} \equiv \tilde{\mathcal{G}}_{\mathcal{A}}, \\
& \left(\operatorname{id}_{\mathrm{G}} \times{ }^{M} \tilde{L}_{\chi}\right)^{*} \tilde{\Upsilon}_{\chi \mathcal{A}} \equiv\left(\operatorname{id}_{\mathrm{G}} \times{ }^{M} \tilde{L}_{\chi}\right)^{*}\left(\Upsilon_{[1,3]^{*}} \otimes \mathrm{id}\right)=\Upsilon_{[1,3]^{*}} \otimes \mathrm{id} \equiv \tilde{\Upsilon}_{\mathcal{A}}, \\
& \left(\operatorname{id}_{\mathrm{G}^{2}} \times{ }^{M} \tilde{L}_{\chi}\right)^{*} \tilde{\gamma}_{\chi \mathcal{A}} \equiv\left(\operatorname{id}_{\mathrm{G}^{2}} \times{ }^{M} \tilde{L}_{\chi}\right)^{*}\left(\gamma_{[1,2,4]^{*}} \otimes \mathrm{id}\right)=\gamma_{[1,2,4]^{*}} \otimes \mathrm{id} \equiv \tilde{\gamma}_{\mathcal{A}},
\end{aligned}
$$

the 1-cell is found to be

$$
\left(\tilde{\Psi}_{\mathcal{A}, \chi}, \tilde{\mathrm{Z}}_{\mathcal{A}, \chi}\right)=\left(\mathrm{id}, \lambda_{\Upsilon_{[1,3]^{*} \otimes \mathrm{id}}^{-1}} \bullet \rho_{\Upsilon_{[1,3]^{*} \otimes \mathrm{id}}}\right),
$$

and the coherence condition (8.4) readily follows from the naturality of the 2-isomorphisms $\lambda \Upsilon_{[1,3]^{*} \otimes \text { id }}$ and $\rho \Upsilon_{[1,3]^{*}} \otimes$ id in $\Upsilon_{[1,3]^{*}} \otimes \mathrm{id}$, as demonstrated in Ref. Whal07a, Lemma 2.3.1]. The manifest G-equivariance of the map ${ }^{M} \tilde{L}_{\chi}$ further implies that the gerbe ${ }^{M} L_{\chi}^{*} \mathcal{G}_{\chi \mathcal{A}}$ is actually the descendant of ${ }^{M} \tilde{L}_{\chi}^{*} \tilde{\mathcal{G}}_{\chi_{\mathcal{A}}}$, and so, upon invoking Theorem 8.10, we infer from Eq. (10.31)

$$
\Psi_{\mathcal{A}, \chi}=\operatorname{id}_{\mathcal{G}_{\mathcal{A}}}
$$


or, equivalently, the equality

$$
{ }^{M} L_{\chi}^{*} \mathcal{G}_{\chi \mathcal{A}}=\mathcal{G}_{\mathcal{A}}
$$

In the next step, we compare the 1-isomorphisms ${ }^{Q} L_{\chi}^{*} \Phi_{\chi_{\mathcal{A}}}$ and $\Phi_{\mathcal{A}}$, a task which - once more by virtue of Theorem 8.10 - reduces to examining the respective parent 1-isomorphisms $\left({ }^{Q} \tilde{L}_{\chi}^{*} \tilde{\Phi}_{\chi_{\mathcal{A}}},\left(\operatorname{id}_{\mathrm{G}} \times{ }^{Q} \tilde{L}_{\chi}\right)^{*} \tilde{\Xi}_{\chi \mathcal{A}} ; 0\right)$ and $\left(\tilde{\Phi}_{\mathcal{A}}, \tilde{\Xi}_{\mathcal{A}} ; 0\right)$ from $\mathfrak{B} \mathfrak{G r} \mathfrak{b} \nabla(\tilde{Q})_{0}^{\mathrm{G}}$. An explicit calculation:

$$
\begin{aligned}
& { }^{Q} \tilde{L}_{\chi}^{*} \tilde{\Phi}_{\chi \mathcal{A}} \equiv{ }^{Q} \tilde{L}_{\chi}^{*}\left(\Phi_{2^{*}} \otimes J_{\tilde{\lambda}_{\chi_{\mathcal{A}}}}\right)=\Phi_{2^{*}} \otimes J_{Q_{\tilde{L}_{\chi}^{*}} \tilde{\lambda}_{\chi_{\mathcal{A}}}}=\Phi_{2^{*}} \otimes J_{\tilde{\lambda}_{\lambda_{\chi}^{*} \chi_{\mathcal{A}}}}=\Phi_{2^{*}} \otimes J_{\tilde{\lambda}_{\mathcal{A}}} \equiv \tilde{\Phi}_{\mathcal{A}}, \\
& \left(\operatorname{id}_{\mathrm{G}} \times{ }^{Q} \tilde{L}_{\chi}\right)^{*} \tilde{\Xi}_{\chi \mathcal{A}} \equiv\left(\operatorname{id}_{\mathrm{G}} \times{ }^{Q} \tilde{L}_{\chi}\right)^{*}\left(\Xi_{[1,3]^{*}} \otimes \mathrm{id}\right)=\Xi_{[1,3]^{*}} \otimes \mathrm{id} \equiv \tilde{\Xi}_{\mathcal{A}}
\end{aligned}
$$

yields the equality

$$
{ }^{Q} L_{\chi}^{*} \Phi_{\chi_{\mathcal{A}}}=\Phi_{\mathcal{A}}
$$

in consequence of the same Theorem 8.10 .

At this stage, it remains to establish a relation between the 2-isomorphisms ${ }^{T_{n}} L_{\chi}^{*} \varphi_{n} \times \mathcal{A}$ and $\varphi_{n \mathcal{A}}$ by examining the parent 2 -isomorphisms ${ }^{T_{n}} \tilde{L}_{\chi}^{*} \tilde{\varphi}_{n} \chi_{\mathcal{A}}$. The latter rewrite as

$$
{ }^{T_{n}} \tilde{L}_{\chi}^{*} \tilde{\varphi}_{n \chi \mathcal{A}} \equiv{ }^{T_{n}} \tilde{L}_{\chi}^{*} \varphi_{n 2^{*}}=\varphi_{n 2^{*}} \equiv \tilde{\varphi}_{n \mathcal{A}},
$$

which, again by virtue of Theorem 8.10, implies the equality

$$
{ }^{T_{n}} L_{\chi}^{*} \varphi_{n \times \mathcal{A}}=\varphi_{n \mathcal{A}}
$$

All in all, we have

$$
\left({ }^{M} L_{\chi}^{*} \mathcal{G}_{\chi_{\mathcal{A}}},{ }^{Q} L_{\chi}^{*} \Phi_{\chi_{\mathcal{A}}},{ }^{T_{n}} L_{\chi}^{*} \varphi_{n \times \mathcal{A}}\right)=\left(\mathcal{G}_{\mathcal{A}}, \Phi_{\mathcal{A}}, \varphi_{n \mathcal{A}}\right),
$$

which immediately implies Eq. (10.30) and thus concludes the proof of the theorem.

\section{The COHOMOlOgicAl CLASSIFICATION OF G-EQUIVARIANT STRING BACKGROUNDS}

The prime rôle played by a G-equivariant structure on the string background of the $\sigma$-model in gauging a rigid G-symmetry and in descending the ensuing model to the quotient of the target space by the action of $\mathrm{G}$ motivate further study of obstructions to the existence of such structures and their classification. A particularly convenient approach to these problems bases on a cohomological presentation of the various components of the background (the gerbe, the bi-brane 1-isomorphism and the inter-bi-brane 2-isomorphism) alongside the 1and 2-morphisms of the G-equivariant structure. Such a presentation uses local data of the geometric structures associated with a judiciously chosen open cover of the simplicial G-space GFF. A proper treatment of the issue prerequires further elaboration of the basic simplicial framework laid out in Section 2.2, which we carry out in Appendix If and put to work in the opening part of the present section. Once the formalism is established, we proceed with a systematic reconstruction of the cohomological description of a G-equivariant background.

11.1. Setting up the local description. The point of departure in a local description of the geometric objects of interest, extending the one of Section 7.1, is a choice of an open cover of the manifolds that support them. In the case in hand, in which the manifolds combine into simplicial G-spaces and definitions of the geometric objects use the attendant face maps, it is natural to render the choice of a cover consistent with the group action. The proper notion is that of aligned simplicial sequences of G-invariant refinements of open covers, as defined in Appendix I. Below, we specialise the general construction presented therein to the setting of interest, thus preparing the tools for later developments.

We shall work with sequences $\left\{{ }^{\mathscr{M}} \mathcal{O}^{m}\right\}_{m=0,1, \ldots}$ of open covers ${ }^{\mathscr{M}} \mathcal{O}^{m}=\left\{{ }^{\mathscr{M}} \mathcal{O}_{i}^{m}\right\}_{i \in \mathscr{M} \mathscr{I} m}$ of the manifolds $\mathscr{M}^{m} \equiv \mathrm{G} \mathscr{M}([m])=\mathrm{G}^{m} \times \mathscr{M}, \mathscr{M}=M, Q, T_{n}$, chosen so that there exist index maps

$$
\phi_{\alpha}^{(m)}:{ }^{Q} \mathscr{I}^{m} \rightarrow^{M} \mathscr{I}^{m}, \quad \quad \psi_{n}^{k, k+1(m)}:{ }^{T_{n}} \mathscr{I}^{m} \rightarrow^{Q} \mathscr{I}^{m}
$$

which cover (or Čech-extend) the corresponding manifold maps in the sense made precise by the conditions

$$
\iota_{\alpha}^{(m)}\left({ }^{Q} \mathcal{O}_{i}^{m}\right) \subset{ }^{M} \mathcal{O}_{\phi_{\alpha}^{(m)}(i)}^{m}, \quad \quad \pi_{n}^{k, k+1(m)}\left({ }^{T_{n}} \mathcal{O}_{i}^{m}\right) \subset{ }^{Q} \mathcal{O}_{\psi_{n}^{k, k+1}(m)}^{m}(i) .
$$


A straightforward application of Proposition I.5 now yields simplicial sequences of refinements $\mathscr{M}^{m}=\left\{\mathscr{M}_{\mathcal{J}}^{m}\right\}_{j \in \mathscr{M}} \mathscr{J}^{m}$ of the ${ }^{\mathscr{M}} \mathcal{O}^{m}$ engendered by the G $\mathscr{M}$ and indexed by the correspond-

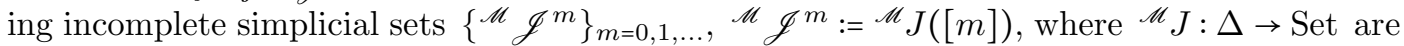
the contravariant functors from Proposition I.2. The functors are related by the natural transformations of Proposition I.5,

$$
j_{\alpha}:{ }^{Q} J \rightarrow{ }^{M} J, \quad j_{n}^{k, k+1}:{ }^{T_{n}} J \rightarrow{ }^{M} J,
$$

with $j_{\alpha}^{(m)}:=j_{\alpha}([m])$ and $j_{n}^{k, k+1(m)}:=j_{n}^{k, k+1}([m])$ such that the covering relations

$$
\iota_{\alpha}^{(m)}\left({ }^{Q} \mathcal{U}_{\jmath}^{m}\right) \subset{ }^{M} \mathcal{U}_{j_{\alpha}^{(m)}(\jmath)}^{m}, \quad \quad \pi_{n}^{k, k+1(m)}\left({ }^{T_{n}} \mathcal{U}_{\jmath}^{m}\right) \subset{ }^{Q} \mathcal{U}_{j_{n}^{k, k+1}(m)}^{m}(\jmath)
$$

hold true, and the diagram

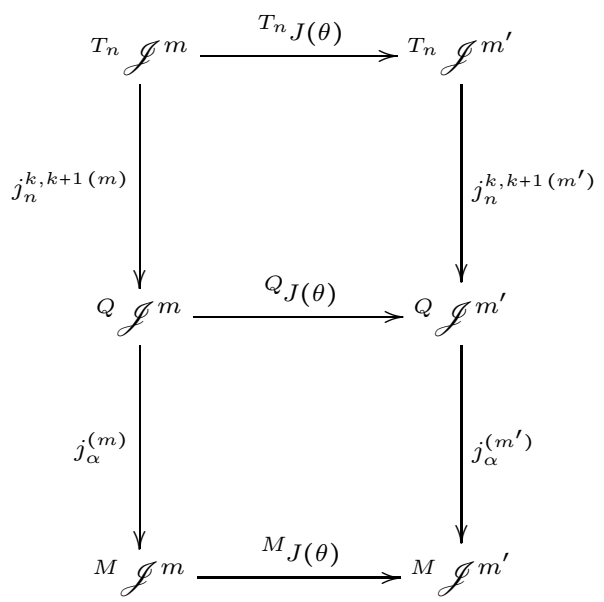

is commutative for any $\theta \in \Delta\left(m^{\prime}, m\right)$.

The foregoing discussion affords a particularly compact description of the Čech cohomology of differential sheaves over the simplicial G-manifolds G $\mathscr{M}$. Indeed, we may associate with the simplicial covers $\mathscr{M}^{m}{ }^{m}$ a number of Čech-extended maps (in the sense of Ref. [RS09]), namely, the 'horizontal' ones: ${ }^{\mathscr{M}} \breve{d}_{k}^{(m)}:=\left({ }^{\mathscr{M}} d_{k}^{(m)}, \mathscr{M}_{k}^{(m)}\right), \mathscr{M}_{k}^{(m)}:=\mathscr{M}^{(m}\left(\theta_{k}^{(m)}\right)$, and the 'vertical' ones: $\check{\iota}_{\alpha}^{(m)}:=\left(\iota_{\alpha}^{(m)}, j_{\alpha}^{(m)}\right)$ and $\check{\pi}_{n}^{k, k+1(m)}:=\left(\pi_{n}^{k, k+1(m)}, j_{n}^{k, k+1(m)}\right)$. These may subsequently be used to pull back sections of differential sheaves. Let $\mathcal{S}_{\mathscr{M}^{m}}^{0}=\underline{2 \pi \mathbb{Z}}_{\mathscr{M}^{m}}$ be the sheaf of locally constant $2 \pi \mathbb{Z}$-valued functions on $\mathscr{M}^{m}$, and $\mathcal{S}_{\mathscr{M}^{m}}^{p+1}=\underline{\Omega}^{p}\left(\mathscr{M}^{m}\right), p \geq 0$ the sheaves of locally smooth (real) $p$-forms on the same space. Take an arbitrary section $s \in \Gamma\left(\mathcal{S}_{\mathscr{M}^{m}}^{p}\right)$ given as a collection $s=\left(s_{\jmath}\right)_{\jmath \in \mathscr{M}_{\mathscr{J}} m}$. We define its pullback along $\mathscr{M}_{\mathrm{d}_{k}}^{(m)}$ by the formula

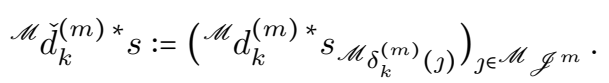

Extending this prescription to arbitrary $\mathcal{S}_{\mathscr{M}^{m}}^{p}$-valued Čech $q$-cochains, we obtain maps

$$
\mathscr{M}_{\bar{d}}^{(m) *}: \check{C}^{q}\left(\mathscr{M}^{m-1}, \mathcal{S}_{\mathscr{M}^{m-1}}^{p}\right) \rightarrow \check{C}^{q}\left(\mathscr{M}^{m}, \mathcal{S}_{\mathscr{M}^{m}}^{p}\right)
$$

satisfying the cosimplicial identities

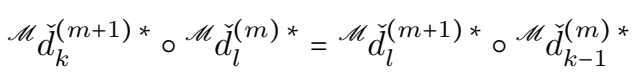

for all $0 \leq l<k$ and $k=0,1,2, \ldots, m+1$. These imply the cohomological identity

$$
\mathscr{M}_{\mathrm{G}, r}^{(m)} \circ \mathscr{M} \check{\delta}_{\mathrm{G}, r}^{(m-1)}=0
$$

for the coboundary operators

$$
\mathscr{M}_{\mathrm{G}, r}^{(m)}:=\sum_{k=0}^{m+1}(-1)^{m+1-k} \mathscr{M}_{k}^{(m+1) *}: A^{r}\left(\mathscr{M}_{\mathcal{U}^{m}}\right) \rightarrow A^{r}\left(\mathscr{M}_{\mathcal{U}^{m+1}}\right)
$$

defined on the cochain groups

$$
A^{r}\left(\mathscr{M}^{m}\right):=\oplus_{p+q=r} \check{C}^{q}\left(\mathscr{M}^{m}, \mathcal{D}(4)_{\mathscr{M}^{m}}^{p}\right), \quad \mathcal{D}(4)_{\mathscr{M}^{m}}^{p}:=\mathcal{S}_{\mathscr{M}^{m}}^{p}
$$


of the Čech-Deligne bicomplex obtained as an extension of the Deligne complex

$$
\mathcal{D}(4)_{\mathscr{M}^{m}}: 0 \rightarrow \mathcal{S}_{\mathscr{M}^{m}}^{0} \stackrel{\mathrm{d}^{(0)}:=\mathrm{id} \mathcal{S}_{\mathcal{M}^{m}}^{0}}{\longrightarrow} \mathcal{S}_{\mathscr{M}^{m}}^{1} \stackrel{\mathrm{d}^{(1)}:=\mathrm{d}}{\longrightarrow} \mathcal{S}_{\mathscr{M}^{m}}^{2} \stackrel{\mathrm{d}^{(2)}:=\mathrm{d}}{\longrightarrow} \mathcal{S}_{\mathscr{M}^{m}}^{3} \stackrel{\mathrm{d}^{(3)}:=\mathrm{d}}{\longrightarrow} \mathcal{S}_{\mathscr{M}^{m}}^{4},
$$

cf. Eq. (7.1), through Čech cohomology of the form

$$
0 \rightarrow \check{C}^{0}\left(\mathscr{M}^{m}, \mathcal{S}_{\mathscr{M}^{m}}^{p}\right) \stackrel{\check{\delta}^{(0)}}{\longrightarrow} \check{C}^{1}\left({ }^{\mathscr{M}} \mathcal{U}^{m}, \mathcal{S}_{\mathscr{M}^{m}}^{p}\right) \stackrel{\check{\delta}^{(1)}}{\longrightarrow} \check{C}^{2}\left({ }^{\mathscr{M}} \mathcal{U}^{m}, \mathcal{S}_{\mathscr{M}^{m}}^{p}\right) \stackrel{\check{\delta}^{(2)}}{\longrightarrow \cdots} .
$$

Here, the $\check{\delta}^{(q)}$ are the standard Čech coboundary operators

$$
\begin{aligned}
\check{\delta}^{(q)} \quad: \quad \check{C}^{q}\left(\mathscr{M}^{m} \mathcal{U}^{m}, \mathcal{S}_{\mathscr{M}^{m}}^{p}\right) \rightarrow \check{C}^{q+1}\left(\mathscr{M}^{m}, \mathcal{S}_{\mathscr{M}^{m}}^{p}\right) \\
: \quad\left(s_{i_{0} i_{1} \ldots i_{q}}\right) \mapsto\left(\left(\check{\delta}^{(q)} s\right)_{i_{0} i_{1} \ldots i_{q+1}}\right):=\left(\left.\sum_{k=0}^{q+1}(-1)^{k} s_{i_{0} i_{1} \ldots i_{\bar{i}_{k}}}\right|_{\mathscr{M}_{\mathcal{O}_{i_{0} i_{1} \ldots i_{q+1}}}}\right),
\end{aligned}
$$

which we use to define the Deligne differentials ${ }^{\mathscr{M}} D_{r}^{(m)}$ with restrictions

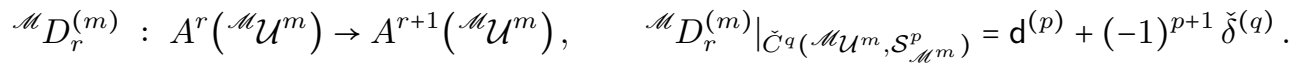

The corresponding Deligne (hyper-)cohomology groups are denoted as

$$
\mathbb{H}^{r}\left(\mathscr{M}^{m}, \mathcal{D}(4)^{\bullet} \dot{\mathscr{M}}^{m}\right):=\frac{\operatorname{ker}^{\mathscr{M}} D_{r}^{(m)}}{\operatorname{im} \mathscr{M}_{r-1}^{(m)}} .
$$

We have the important isomorphisms

$$
\begin{gathered}
\mathbb{H}^{3}\left(\mathscr{M}^{m}, \mathcal{D}(4)_{\mathscr{M}^{m}}\right) \cong H^{2}\left(\mathscr{M}^{m}, \mathrm{U}(1)\right), \quad \mathbb{H}^{2}\left(\mathscr{M}^{m}, \mathcal{D}(4)_{\mathscr{M}^{m}}\right) \cong H^{1}\left(\mathscr{M}^{m}, \mathrm{U}(1)\right) \\
\mathbb{H}^{1}\left(\mathscr{M}^{m}, \mathcal{D}(4)_{\mathscr{M}^{m}}^{\bullet} \cong H^{0}\left(\mathscr{M}^{m}, \mathrm{U}(1)\right) \cong \mathrm{U}(1)^{\pi_{0}\left(\mathscr{M}_{m}\right)}\right.
\end{gathered}
$$

The coboundary operators $\mathscr{M}_{\mathrm{G}, r}^{(m)}$ commute with Deligne differentials,

$$
\mathscr{M}_{\mathrm{G}, r+1}^{(m)} \circ{ }^{\mathscr{M}} D_{r}^{(m)}={ }^{\mathscr{M}} D_{r}^{(m+1)} \circ \mathscr{M}_{\tilde{\delta}_{\mathrm{G}, r}^{(m)}},
$$

and so they induce cohomology maps (denoted by the same symbols)

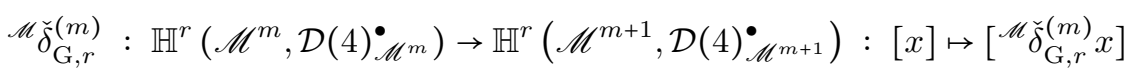

that will be used amply in what follows.

Analogously, we define maps

$$
\check{\iota}_{\alpha}^{(m) *}: \check{C}^{q}\left({ }^{M} \mathcal{U}^{m}, \mathcal{S}\right) \rightarrow \check{C}^{q}\left({ }^{Q} \mathcal{U}^{m}, \mathcal{S}\right), \quad \check{\pi}_{n}^{k, k+1(m) *}: \check{C}^{q}\left({ }^{Q} \mathcal{U}^{m}, \mathcal{S}\right) \rightarrow \check{C}^{q}\left({ }^{T_{n}} \mathcal{U}^{m}, \mathcal{S}\right),
$$

and their distinguished linear combinations

$$
\begin{aligned}
& \check{\Delta}_{Q, r}^{(m)}:=\check{\iota}_{2}^{(m) *}-\check{\iota}_{1}^{(m) *}: A^{r}\left({ }^{M} \mathcal{U}^{m}\right) \rightarrow A^{r}\left({ }^{Q} \mathcal{U}^{m}\right), \\
& \check{\Delta}_{T_{n}, r}^{(m)}:=\sum_{k=1}^{n} \varepsilon_{n}^{k, k+1} \check{\pi}_{n}^{k, k+1(m) *}: A^{r}\left({ }^{Q} \mathcal{U}^{m}\right) \rightarrow A^{r}\left({ }^{T_{n}} \mathcal{U}^{m}\right) .
\end{aligned}
$$

These satisfy the identities

$$
\check{\Delta}_{T_{n}, r}^{(m)} \circ \check{\Delta}_{Q, r}^{(m)}=0,
$$

cf. Eq. (2.5).

Clearly, all the above pullback operators intertwine the respective Deligne differentials,

$$
\check{\Delta}_{Q, r+1}^{(m)} \circ{ }^{M} D_{r}^{(m)}={ }^{Q} D_{r}^{(m)} \circ \check{\Delta}_{Q, r}^{(m)}, \quad \check{\Delta}_{T_{n}, r+1}^{(m)} \circ{ }^{Q} D_{r}^{(m)}={ }^{T_{n}} D_{r}^{(m)} \circ \check{\Delta}_{T_{n}, r}^{(m)},
$$

and so they give rise to cohomology maps (denoted by the same symbols)

$$
\begin{array}{lll}
\check{\Delta}_{Q, r}^{(m)} & : & \mathbb{H}^{r}\left(\mathrm{G}^{m} \times M, \mathcal{D}(4)_{\mathrm{G}^{m} \times M}^{\bullet}\right) \rightarrow \mathbb{H}^{r}\left(\mathrm{G}^{m} \times Q, \mathcal{D}(4)_{\mathrm{G}^{m} \times Q}^{{ }^{m} \times Q}\right):[x] \mapsto\left[\check{\Delta}_{Q . r}^{(m)} x\right], \\
\check{\Delta}_{T_{n}, r}^{(m)} & : & \mathbb{H}^{r}\left(\mathrm{G}^{m} \times Q, \mathcal{D}(4)_{\mathrm{G}^{m} \times Q}^{\bullet}\right) \rightarrow \mathbb{H}^{r}\left(\mathrm{G}^{m} \times T_{n}, \mathcal{D}(4)_{\mathrm{G}^{m} \times T_{n}}\right):[x] \mapsto\left[\check{\Delta}_{T_{n}, r}^{(m)} x\right] .
\end{array}
$$


The mutual commutation relations between the 'horizontal' and the 'vertical' pullback operators can be succinctly expressed by the commutative diagram

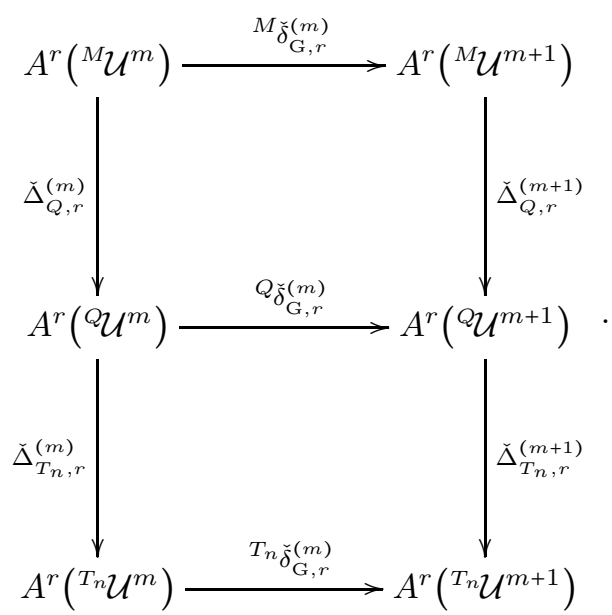

We are now fully equipped to reconstruct a local description of a G-equivariant string background.

As a first step, we give a local presentation of the background $\mathfrak{B}=(\mathcal{M}, \mathcal{B}, \mathcal{J})$ from Definition 2.1. Thus, the gerbe $\mathcal{G}$ is presented by a 3-cochain

$$
\mathcal{G} \stackrel{\text { loc. }}{\longrightarrow} b \in A^{3}\left({ }^{M} \mathcal{U}^{0}\right)
$$

satisfying the cohomological identity

$$
{ }^{M} D_{3}^{(0)} b=\overline{\mathrm{H}}, \quad \overline{\mathrm{H}}:=\left(\left.\mathrm{H}\right|_{{ }_{{ }_{\mathcal{U}}}^{0}}, 0,0,0,0\right) .
$$

1-isomorphism classes of local data of $\mathcal{G}$ with arbitrary curvature (collecting local data of $\mathcal{G}$ and of gerbes 1-isomorphic to $\mathcal{G}$ ) are enumerated by elements of the hyper-cohomology group $\mathbb{H}^{3}\left(M, \mathcal{D}(3)_{M}^{\bullet}\right)$, with the Deligne complex $\mathcal{D}(3)_{M}^{\bullet}$ defined similarly as $\mathcal{D}(4)_{M}^{\bullet}$ but without the terminal node $\mathcal{D}(4)_{M}^{4}$. To the $\mathcal{G}$-bi-brane 1-isomorphism $\Phi$, we associate a 2-cochain

$$
\Phi \stackrel{\text { loc. }}{\longrightarrow} p \in A^{2}\left({ }^{Q} \mathcal{U}^{0}\right)
$$

subject to the identity

$$
{ }^{Q} D_{2}^{(0)} p=\check{\Delta}_{Q, 3}^{(0)} b+\bar{\omega}, \quad \bar{\omega}=\left(\left.\omega\right|_{Q \mathcal{U}_{i}^{0}}, 0,0,0\right),
$$

cf. Eq. (7.4). Finally, the inter-bi-brane 2-isomorphisms $\varphi_{n}$ define the respective 1-cochains

$$
\varphi_{n} \stackrel{\text { loc. }}{\longrightarrow} h_{n} \in A^{1}\left({ }^{T_{n}} \mathcal{U}^{0}\right)
$$

obeying the identities

$$
{ }^{T_{n}} D_{1}^{(0)} h_{n}=-\check{\Delta}_{T_{n}, 2}^{(0)} p,
$$

cf. Eq. (7.5).

Next, we write out local data of a $(\mathrm{G}, \rho)$-equivariant structure $(\Upsilon, \gamma ; \kappa)$ on $\mathcal{G}$. These consist of a 2-cochain describing the 1-isomorphism $\Upsilon$,

$$
\Upsilon \stackrel{\text { loc. }}{\longrightarrow} a \in A^{2}\left({ }^{M} \mathcal{U}^{1}\right),
$$

a 1-cochain induced by the 2-isomorphism $\gamma$,

$$
\gamma \stackrel{\text { loc. }}{\longrightarrow} e \in A^{1}\left({ }^{M} \mathcal{U}^{2}\right),
$$


and a 0 -cochain $\nu \in A^{0}\left({ }^{M} \mathcal{U}^{3}\right)$ that encodes the coherence constraint (8.4). They are required to satisfy the identities

$$
\begin{aligned}
{ }^{M} D_{2}^{(1)} a & =-{ }^{M} \check{\delta}_{\mathrm{G}, 3}^{(0)} b+\bar{\rho}, \quad \bar{\rho}=\left(\left.\rho\right|_{M_{\mathcal{U}_{i}^{1}}}, 0,0,0\right), \\
{ }^{M} D_{1}^{(2)} e & =-{ }^{M} \check{\delta}_{\mathrm{G}, 2}^{(1)} a \\
{ }^{M} D_{0}^{(3)} \nu & ={ }^{M} \check{\delta}_{\mathrm{G}, 1}^{(2)} e \\
{ }^{M} \check{\delta}_{\mathrm{G}, 0}^{(3)} \nu & =0 .
\end{aligned}
$$

Passing to local data of a $(\mathrm{G}, \lambda)$-equivariant structure $(\Xi ; k)$ on $\mathcal{B}$, we find a 1-cochain representing the 2-isomorphism $\Xi$ as

$$
\Xi \stackrel{\text { loc. }}{\longrightarrow} s \in A^{1}\left(\mathcal{Q}^{1}\right)
$$

alongside a 0 -cochain $\theta \in A^{0}\left({ }^{\mathcal{U}}{ }^{2}\right)$ that accounts for the coherence condition (8.8). They verify the identities

$$
\begin{aligned}
{ }^{Q} D_{1}^{(1)} s & =-{ }^{Q} \check{\delta}_{\mathrm{G}, 2}^{(0)} p-\check{\Delta}_{Q, 2}^{(1)} a+\bar{\lambda}, \quad \bar{\lambda}=\left(\left.\lambda\right|_{Q_{\mathcal{U}_{i}^{1}}}, 0,0\right), \\
{ }^{Q} D_{0}^{(2)} \theta & ={ }^{Q} \check{\delta}_{\mathrm{G}, 1}^{(1)} s-\check{\Delta}_{Q, 1}^{(2)} e, \\
{ }^{Q} \check{\delta}_{\mathrm{G}, 0}^{(2)} \theta & =-\check{\Delta}_{Q, 0}^{(3)} \nu .
\end{aligned}
$$

Finally, we can rephrase the defining relation (8.10) of a G-equivariant structure on $\mathcal{J}$ in terms of a 0 -cochain $\varsigma \in A^{0}\left({ }^{T_{n}} \mathcal{U}^{1}\right)$ subject to the cohomological identities

$$
\begin{aligned}
{ }^{T_{n}} D_{0}^{(1)} \varsigma & ={ }^{T_{n}} \check{\delta}_{\mathrm{G}, 1}^{(0)} h_{n}-\check{\Delta}_{T_{n}, 1}^{(1)} s, \\
{ }^{T_{n}} \check{\delta}_{\mathrm{G}, 0}^{(1)} \varsigma & =-\check{\Delta}_{T_{n}, 0}^{(2)} \theta .
\end{aligned}
$$

Since ${ }^{\mathscr{M}} D_{0}^{(\bullet)}$ is injective, relations (11.7), (11.10) and (11.12) follow from the preceding ones, so they are spurious. We may now examine cohomological conditions for the existence of a G-equivariant structure on a fixed string background.

11.2. (G, $\rho$ )-equivariant gerbes. We shall first consider circumstances in which a gerbe $\mathcal{G}$ can be endowed with a G-equivariant structure.

11.2.1. Obstructions to the existence of $\Upsilon$ and $\gamma$. Given a 3-cochain $b \in A^{3}\left({ }^{M} \mathcal{U}^{0}\right)$ on the target space $M$, representing, as in Eq. (11.1), a gerbe $\mathcal{G}$ with a G-invariant curvature $\mathrm{H}$ satisfying the identity

$$
{ }^{M} \check{\delta}_{\mathrm{G}, 4}^{(0)} \overline{\mathrm{H}}={ }^{M} D_{3}^{(1)} \bar{\rho},
$$

cf. Eq. (2.39), we find through direct calculation

$$
{ }^{M} D_{3}^{(1)}{ }^{M} \check{\delta}_{\mathrm{G}, 3}^{(0)} b={ }^{M} \check{\delta}_{\mathrm{G}, 4}^{(0)}{ }^{M} D_{3}^{(0)} b={ }^{M} \check{\delta}_{\mathrm{G}, 4}^{(0)} \overline{\mathrm{H}}={ }^{M} D_{3}^{(1)} \bar{\rho},
$$

that the 3 -cochain ${ }^{M} \check{\delta}_{\mathrm{G}, 3}^{(0)} b-\bar{\rho} \in A^{3}\left({ }^{M} \mathcal{U}^{(1)}\right)$ defines a class

$$
\left[{ }^{M} \check{\delta}_{\mathrm{G}, 3}^{(0)} b-\bar{\rho}\right] \in H^{2}(\mathrm{G} \times M, \mathrm{U}(1)) .
$$

This is the class of the flat gerbe $\mathcal{D} \cong{ }^{M} \ell^{*} \mathcal{G} \otimes \mathcal{G}_{2 *}^{\vee} \otimes I_{-\rho}$ of Proposition 4.2, obstructing the existence of a 1-isomorphism $\Upsilon:{ }^{M} \ell^{*} \mathcal{G} \rightarrow \mathcal{G}_{2 *} \otimes I_{\rho}$. Decomposing the relevant cohomology group with the help of the Universal Coefficient Theorem and the Künneth Theorem as

$H^{2}(\mathrm{G} \times M, \mathrm{U}(1)) \cong H^{2}(\mathrm{G}, \mathrm{U}(1))^{\pi_{0}(M)} \oplus \operatorname{Hom}_{\mathbb{Z}}\left(H_{1}(\mathrm{G}) \otimes H_{1}(M), \mathrm{U}(1)\right) \oplus H^{2}(M, \mathrm{U}(1))^{\pi_{0}(\mathrm{G})}$, we arrive at

\footnotetext{
${ }^{5}$ We are representing various canonical 2-isomorphisms entering the definition of $\gamma^{\sharp}$ by trivial local data.
} 
Proposition 11.1. GSW10, Prop. 6.1] The class [D] $\in H^{2}(\mathrm{G} \times M, \mathrm{U}(1))$ that obstructs the existence of a 1-isomorphism $\Upsilon$ of a $(\mathrm{G}, \rho)$-equivariant structure on $\mathcal{G}$ decomposes as a direct sum

$$
[\mathcal{D}]=[\mathcal{D}]_{2,0} \oplus[\mathcal{D}]_{1,1} \oplus[\mathcal{D}]_{0,2}
$$

of terms from the subspaces $H^{2}(\mathrm{G}, \mathrm{U}(1))^{\pi_{0}(M)} \ni[\mathcal{D}]_{2,0}, \operatorname{Hom}_{\mathbb{Z}}\left(H_{1}(\mathrm{G}) \otimes H_{1}(M), \mathrm{U}(1)\right) \ni$ $[\mathcal{D}]_{1,1}$ and $H^{2}(M, \mathrm{U}(1))^{\pi_{0}(\mathrm{G})} \ni[\mathcal{D}]_{0,2}$.

As a particular case, we obtain

Corollary 11.2. GSW10, Cor.6.2] If the connected components of $\mathrm{G}$ and $M$ are 2connected, there is no obstruction to the existence of a 1-isomorphism $\Upsilon$.

Assume, next, that the first obstruction has been lifted, i.e. that there exists, for $\left\{{ }^{M} \mathcal{U}^{n}\right\}$ sufficiently fine, a 2-cochain $a \in A^{2}\left({ }^{M} \mathcal{U}^{1}\right)$ such that Eq. (11.4) holds. The 2-cochain provides local data of a 1-isomorphism $\Upsilon$. Taking into account the identity

$$
{ }^{M} \check{\delta}_{\mathrm{G}, 3}^{(1)} \bar{\rho}=0,
$$

tantamount to Eq. (2.38), we readily establish that

$$
{ }^{M} D_{2}^{(2) M} \check{\delta}_{\mathrm{G}, 2}^{(1)} a={ }^{M} \check{\delta}_{\mathrm{G}, 3}^{(1) M} D_{2}^{(1)} a=-{ }^{M} \check{\delta}_{\mathrm{G}, 3}^{(1) M} \check{\delta}_{\mathrm{G}, 3}^{(0)} b+{ }^{M} \check{\delta}_{\mathrm{G}, 3}^{(1)} \bar{\rho}=0 .
$$

We conclude that ${ }^{M} \check{\delta}_{\mathrm{G}, 2}^{(1)} a$ defines a class

$$
\left[{ }^{M} \check{\delta}_{\mathrm{G}, 2}^{(1)} a\right] \in H^{1}\left(\mathrm{G}^{2} \times M, \mathrm{U}(1)\right)
$$

that obstructs the existence of a 2 -isomorphism $\gamma:\left({ }^{M} d_{0}^{(2) *} \Upsilon \otimes \mathrm{id}\right) \circ{ }^{M} d_{2}^{(2) *} \Upsilon \Rightarrow{ }^{M} d_{1}^{(2) *} \Upsilon$. Given that $a$ is determined by Eq. (11.4) only up to shifts

$$
a \mapsto a+a^{\prime}, \quad a^{\prime} \in \operatorname{ker}{ }^{M} D_{2}^{(1)},
$$

capturing the freedom of choice of (local data for) $\Upsilon$, we infer that the above class is defined modulo elements of the group

$$
{ }^{M} \mathcal{H}^{1,2}:={ }^{M} \check{\delta}_{\mathrm{G}, 2}^{(1)}\left(H^{1}(\mathrm{G} \times M, \mathrm{U}(1))\right) .
$$

In this manner, we establish

Proposition 11.3. GSW10, Prop. 6.3] Let $a \in A^{2}\left({ }^{M} \mathcal{U}^{1}\right)$ be local data of a 1-isomorphism $\Upsilon:{ }^{M} \ell^{*} \mathcal{G} \rightarrow \mathcal{G}_{2 *} \otimes I_{\rho}$. Then, there exists a 2-isomorphism $\gamma:\left({ }^{M} d_{0}^{(2){ }^{*}} \Upsilon \otimes \mathrm{id}\right) \circ{ }^{M} d_{2}^{(2)}{ }^{*} \Upsilon \Rightarrow$ ${ }^{M} d_{1}^{(2) *} \Upsilon$ iff the obstruction class

$$
\left[{ }^{M} \check{\delta}_{\mathrm{G}, 2}^{(1)} a\right]+{ }^{M} \mathcal{H}^{1,2} \in H^{1}\left(\mathrm{G}^{2} \times M, \mathrm{U}(1)\right) /{ }^{M} \mathcal{H}^{1,2}
$$

is trivial.

We have, in particular,

Corollary 11.4. GSW10, Cor.6.4] If the connected components of $\mathrm{G}$ and $M$ are 1connected, there is no obstruction to the existence of a 2-isomorphism $\gamma$.

One may get a more precise understanding of the structure of the obstruction by decomposing the cohomology groups,

$$
\begin{aligned}
& H^{1}(\mathrm{G} \times M, \mathrm{U}(1)) \cong H^{1}(\mathrm{G}, \mathrm{U}(1))^{\pi_{0}(M)} \oplus H^{1}(M, \mathrm{U}(1))^{\pi_{0}(\mathrm{G})} \\
& H^{1}\left(\mathrm{G}^{2} \times M, \mathrm{U}(1)\right) \\
& \cong H^{1}(\mathrm{G}, \mathrm{U}(1))^{\pi_{0}(\mathrm{G}) \times \pi_{0}(M)} \oplus H^{1}(\mathrm{G}, \mathrm{U}(1))^{\pi_{0}(\mathrm{G}) \times \pi_{0}(M)} \oplus H^{1}(M, \mathrm{U}(1))^{\pi_{0}(\mathrm{G})^{2}},
\end{aligned}
$$

and examining in detail the image of the former in the latter under ${ }^{M} \check{\delta}_{\mathrm{G}, 2}^{(1)}$. This way, one finds, among other things,

Corollary 11.5. GSW10, Cor.6.5] On the G-space $M:=\mathscr{G}$ in the WZW-model context of Definition 5.1, a suitable choice of a 1-isomorphism $\Upsilon$ ensures the existence of a 2isomorphism $\gamma$. 
In the last step, we presuppose that obstructions to the existence of $(\Upsilon, \gamma)$ have both been lifted, so that there exists, besides the 2-cochain a introduced formerly, a 1-cochain $e \in A^{1}\left({ }^{M} \mathcal{U}^{2}\right)$ that satisfies relation Eq. (11.5). We now find

$$
{ }^{M} D_{1}^{(3)}{ }^{M} \check{\delta}_{\mathrm{G}, 1}^{(2)} e={ }^{M} \check{\delta}_{\mathrm{G}, 2}^{(2) M} D_{1}^{(2)} e=-{ }^{M} \check{\delta}_{\mathrm{G}, 2}^{(2)} \check{\check{\delta}}_{\mathrm{G}, 2}^{(1)} a=0,
$$

whence we conclude that to ${ }^{M} \check{\delta}_{\mathrm{G}, 1}^{(2)} e$ there is associated a class

$$
\left[{ }^{M} \check{\delta}_{\mathrm{G}, 1}^{(2)} e\right] \in H^{0}\left(\mathrm{G}^{3} \times M, \mathrm{U}(1)\right) .
$$

This is the class of a locally constant map $d={ }^{\mathscr{M}} d_{2}^{(3) *} \gamma \bullet\left(\left(\mathscr{M}_{0}^{(3) *} \gamma \otimes \mathrm{id}\right) \circ \mathrm{id}\right) \bullet\left(\mathrm{id}{ }^{\mathscr{M}} d_{3}^{(3) *} \gamma^{-1}\right) \bullet$ (id $\circ{ }^{\mathscr{M}} d_{1}^{(3) *} \gamma^{-1}$ ) that captures the last obstruction to the existence of a full-blown $(\mathrm{G}, \rho)$ equivariant structure on $\mathcal{G}$, namely the obstruction to the commutativity of diagram (H.1). Under changes of $a$ and $e$ admitted by their respective definitions (and chosen such as to preserve the 2-isomorphism class of $\Upsilon$ ),

$$
\begin{aligned}
& e \mapsto e+{ }^{M} \check{\delta}_{\mathrm{G}, 1}^{(1)} e^{\prime}+e^{\prime \prime}, \quad e^{\prime} \in A^{2}\left({ }^{M} \mathcal{U}^{1}\right), \quad e^{\prime \prime} \in \operatorname{ker}{ }^{M} D_{1}^{(1)}, \\
& a \mapsto a-{ }^{M} D_{1}^{(1)} e^{\prime}
\end{aligned}
$$

this class gets shifted as

$$
\left[{ }^{M} \check{\delta}_{\mathrm{G}, 1}^{(2)} e\right] \mapsto\left[{ }^{M} \check{\delta}_{\mathrm{G}, 1}^{(2)} e\right]+\left[{ }^{M} \check{\delta}_{\mathrm{G}, 1}^{(2)} e^{\prime \prime}\right]=\left[{ }^{M} \check{\delta}_{\mathrm{G}, 1}^{(2)} e\right]+{ }^{M} \check{\delta}_{\mathrm{G}, 1}^{(2)}\left[e^{\prime \prime}\right] .
$$

Write

$$
{ }^{{ }^{M}} \mathcal{H}^{0,3}:={ }^{{ }^{M}} \check{\delta}_{\mathrm{G}, 1}^{(2)}\left(H^{0}\left(\mathrm{G}^{2} \times M, \mathrm{U}(1)\right)\right) \subset H^{0}\left(\mathrm{G}^{3} \times M, \mathrm{U}(1)\right) .
$$

The upshot of the above analysis can now be phrased as

Proposition 11.6. GSW10, Prop. 6.6] Let $e \in A^{1}\left({ }^{M} \mathcal{U}^{2}\right)$ be local data of a 2-isomorphism $\gamma:\left({ }^{M} d_{0}^{(2) *} \Upsilon \otimes \mathrm{id}\right) \circ{ }^{M} d_{2}^{(2) *} \Upsilon \Rightarrow{ }^{M} d_{1}^{(2) *} \Upsilon$ for an appropriate choice of a fixed 1-isomorphism $\Upsilon:{ }^{M} \ell^{*} \mathcal{G} \rightarrow \mathcal{G}_{2^{*}} \otimes I_{\rho}$. The 2-isomorphism can be chosen so that it satisfies the coherence condition of Eq. (8.3) iff the obstruction class

$$
\left[{ }^{M} \check{\delta}_{\mathrm{G}, 1}^{(2)} e\right]+{ }^{M} \mathcal{H}^{0,3} \in H^{0}\left(\mathrm{G}^{3} \times M, \mathrm{U}(1)\right) /{ }^{M} \mathcal{H}^{0,3}
$$

is trivial.

It is convenient to rephrase the statement of the last proposition, along the lines of Ref. GSW10, in terms of the cohomology theory of the group $\pi_{0}(\mathrm{G})$ with values in the $\pi_{0}(\mathrm{G})$-module $\mathrm{U}(1)^{\pi_{0}(M)} \cong H^{0}(M, \mathrm{U}(1))$, the module structure being induced from the action of $\mathrm{G}$ on $M$,

$$
\pi_{0}(\mathrm{G}) \times \mathrm{U}(1)^{\pi_{0}(M)} \rightarrow \mathrm{U}(1)^{\pi_{0}(M)}:([g], u) \mapsto[g] . u, \quad[g] . u([m]):=u\left(\left[g^{-1} \cdot m\right]\right) .
$$

The rephrasing is possible owing to the existence of a natural identification between classes in $H^{0}\left(\mathrm{G}^{p} \times M, \mathrm{U}(1)\right)$, viewed as locally constant $\mathrm{U}(1)$-valued functions on $\mathrm{G}^{p} \times M$, and $p$-chains from $C^{p}\left(\pi_{0}(\mathrm{G}), \mathrm{U}(1)^{\pi_{0}(M)}\right)$. The identification is defined by the formula

$$
\begin{gathered}
H^{0}\left(\mathrm{G}^{p} \times M, \mathrm{U}(1)\right) \ni f^{p} \stackrel{M_{\zeta_{p}}}{\longmapsto} u^{p} \in C^{p}\left(\pi_{0}(\mathrm{G}), \mathrm{U}(1)^{\pi_{0}(M)}\right) \\
u_{\left[g_{1}\right],\left[g_{2}\right], \ldots,\left[g_{p}\right]}^{p}([m]):=f^{p}\left(g_{p}^{-1}, g_{p-1}^{-1}, \ldots, g_{1}^{-1}, m\right) .
\end{gathered}
$$

The cohomological maps ${ }^{M} \check{\delta}_{\mathrm{G}, 1}^{(p)}$ are transformed into the coboundary operators of the group cohomology under this identification,

$$
{ }^{M} \zeta_{p+1} \circ{ }^{M} \check{\delta}_{\mathrm{G}, 1}^{(p)}={ }^{M} \delta_{\pi_{0}(\mathrm{G})}^{(p)} \circ{ }^{M} \zeta_{p}
$$

Since

$$
{ }^{M} \check{\delta}_{\mathrm{G}, 1}^{(3)}\left[{ }^{M} \check{\delta}_{\mathrm{G}, 1}^{(2)} e\right]=\left[{ }^{M} \check{\delta}_{\mathrm{G}, 1}^{(3)}{ }^{(3)} \check{\delta}_{\mathrm{G}, 1}^{(2)} e\right]=0,
$$

we obtain, as a result of the above identification, 
Corollary 11.7. GSW10, Cor. 6.7] The image ${ }^{M} \zeta_{3}\left(\left[{ }^{M} \check{\delta}_{\mathrm{G}, 1}^{(2)} e\right]\right)=:{ }^{M} u^{3}$ of the obstruction class $\left[{ }^{M} \check{\delta}_{\mathrm{G}, 1}^{(2)} e\right]$ for a coherent] 2-isomorphism $\gamma$ under the identification of Eq. (11.13) is a $\mathrm{U}(1)^{\pi_{0}(M)}$-valued 3-cocycle of the group $\pi_{0}(\mathrm{G})$, and that of the obstruction coset $\left[{ }^{M} \check{\delta}_{\mathrm{G}, 1}^{(2)} e\right]+$ ${ }^{M} \mathcal{H}^{0,3}$ is the cohomology class $\left[{ }^{M} u^{3}\right] \in H^{3}\left(\pi_{0}(\mathrm{G}), \mathrm{U}(1)^{\pi_{0}(M)}\right)$.

The advantage of working with group cohomology is that it is amenable to explicit computations. There are several general results about it that can be employed in the context in hand. E.g., for the trivial group 1, we have

$$
H^{p}\left(\mathbf{1}, \mathrm{U}(1)^{\pi_{0}(M)}\right)=\mathbf{1}, \quad p \geq 1 .
$$

This gives us

Corollary 11.8. GSW10, Cor.6.8] If the symmetry group G is connected and the 2isomorphism $\gamma$ exists, then it can always be chosen in a coherent form.

In the previous paragraph, we restricted our choice of redefinitions of local data of $\Upsilon$ and $\gamma$ to those that leave the 2-isomorphism class of $\Upsilon$ unaltered. From the point of view of obstruction analysis, it is well justified to allow more general redefinitions with view to further reducing the obstruction cohomology group and thus rendering it possible to put some $(\mathrm{G}, \rho)$-equivariant structure on a given gerbe. Accordingly, let us consider (in addition to the above) transformations of the type

$$
a \mapsto a+a^{\prime}, \quad a^{\prime} \in \operatorname{ker}{ }^{M} D_{2}^{(1)}, \quad e \mapsto e+e^{\prime}, \quad e^{\prime} \in A^{1}\left({ }^{M} \mathcal{U}^{2}\right),
$$

the two cochains being related through

$$
{ }^{M} \check{\delta}_{\mathrm{G}, 2}^{(1)} a^{\prime}=-{ }^{M} D_{1}^{(2)} e^{\prime} .
$$

The last constraint ensures that Eqs. (11.4) and (11.5) still hold true. It fixes $e^{\prime}$ only up to shifts

$$
e^{\prime} \mapsto e^{\prime}+e^{\prime \prime}, \quad e^{\prime \prime} \in \operatorname{ker}{ }^{M} D_{1}^{(2)},
$$

and so we recover a connecting (Bockstein-type) homomorphism

$$
\left[a^{\prime}\right] \stackrel{M_{\mathscr{B}}^{(1)}}{\longmapsto}\left[{ }^{M} \check{\delta}_{\mathrm{G}, 1}^{(2)} e^{\prime}\right]+{ }^{M} \mathcal{H}^{0,3} .
$$

from the kernel of ${ }^{M} \check{\delta}_{\mathrm{G}, 2}^{(1)}$ acting on $H^{1}(\mathrm{G} \times M, \mathrm{U}(1))$ to $H^{0}\left(\mathrm{G}^{3} \times M, \mathrm{U}(1)\right) /{ }^{M} \mathcal{H}^{0,3}$.

Proposition 11.9. The component of the class $\left[{ }^{M} \check{\delta}_{\mathrm{G}, 1}^{(2)} e\right]+{ }^{M} \mathcal{H}^{0,3} \in H^{0}\left(\mathrm{G}^{3} \times M, \mathrm{U}(1)\right) /{ }^{M} \mathcal{H}^{0,3}$, obstructing the existence of a coherent 2-isomorphism $\gamma$, that lies in the image of the connecting homomorphism ${ }^{M} \mathscr{B}_{2}^{(1)}$ of $E q$. (11.15) can be removed by a suitable choice of a 1 isomorphism ${ }^{M} \ell^{*} \mathcal{G} \rightarrow \mathcal{G}_{2 *} \otimes I_{\rho}$.

We may now proceed to classify inequivalent $(\mathrm{G}, \rho)$-structures on a given gerbe, in the sense of Definition 8.2.

11.2.2. Classification. We are interested here in enumerating all inequivalent $(\mathrm{G}, \rho)$-equivariant structures on a gerbe $\mathcal{G}$ over $M$ with local data given by some $b \in A^{3}\left({ }^{M} \mathcal{U}^{0}\right)$. To this end, we should first write out cohomological relations satisfied by local data of a G-equivariant isomorphism $(\Psi, \eta)$ between two such structures $\left(\mathcal{G}, \Upsilon_{\beta}, \gamma_{\beta} ; \rho\right), \beta=1,2$. These data consist

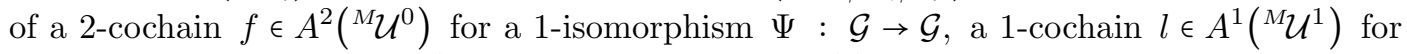
a 2-isomorphism $\eta:\left({ }^{M} d_{0}^{(1) *} \Psi \otimes \mathrm{id}\right) \circ \Upsilon_{1} \Rightarrow \Upsilon_{2} \circ{ }^{M} d_{1}^{(1) *} \Psi$, and a 0 -cochain $\zeta \in A^{0}\left({ }^{M} \mathcal{U}^{2}\right)$ that expresses the coherence of the latter. Denote local data of $\Upsilon_{\beta}$ by $a_{\beta}$ and those of $\gamma_{\beta}$ by $e_{\beta}$ and $\nu_{\beta}$ (the coherence 0-cochain). They obey

${ }^{M} D_{2}^{(1)} a_{\beta}=-{ }^{M} \check{\delta}_{\mathrm{G}, 3}^{(0)} b+\bar{\rho}, \quad{ }^{M} D_{1}^{(2)} e_{\beta}=-{ }^{M} \check{\delta}_{\mathrm{G}, 2}^{(1)} a_{\beta}, \quad{ }^{M} D_{0}^{(3)} \nu_{\beta}={ }^{M} \check{\delta}_{\mathrm{G}, 1}^{(2)} e_{\beta}, \quad{ }^{M} \check{\delta}_{\mathrm{G}, 0}^{(3)} \nu_{\beta}=0$.

Therefore, the respective differences

$$
a_{2,1}:=a_{2}-a_{1}, \quad e_{2,1}:=e_{2}-e_{1}, \quad \nu_{2,1}:=\nu_{2}-\nu_{1}
$$

\footnotetext{
${ }^{6}$ That is one for which the coherence condition of Eq. (8.3) is satisfied.
} 
automatically satisfy the identities

$$
{ }^{M} D_{2}^{(1)} a_{2,1}=0, \quad{ }^{M} D_{1}^{(2)} e_{2,1}=-{ }^{M} \check{\delta}_{\mathrm{G}, 2}^{(1)} a_{2,1}, \quad{ }^{M} D_{0}^{(3)} \nu_{2,1}={ }^{M} \check{\delta}_{\mathrm{G}, 1}^{(2)} e_{2,1}, \quad{ }^{M} \check{\delta}_{\mathrm{G}, 0}^{(3)} \nu_{2,1}=0 .
$$

From Definition 8.2 , we infer that the relations

$$
\begin{array}{cc}
a_{2,1}={ }^{M} \check{\delta}_{\mathrm{G}, 2}^{(0)} f+{ }^{M} D_{1}^{(1)} l, & { }^{M} D_{2}^{(0)} f=0, \\
e_{2,1}=-{ }^{M} \check{\delta}_{\mathrm{G}, 1}^{(1)} l+{ }^{M} D_{0}^{(2)} \zeta, & \nu_{2,1}={ }^{M} \check{\delta}_{\mathrm{G}, 0}^{(2)} \zeta
\end{array}
$$

are tantamount to the existence of a G-equivariant isomorphism between the two $(\mathrm{G}, \rho)$ equivariant structures. Define a bicomplex ${ }^{M} \mathcal{K}$ :

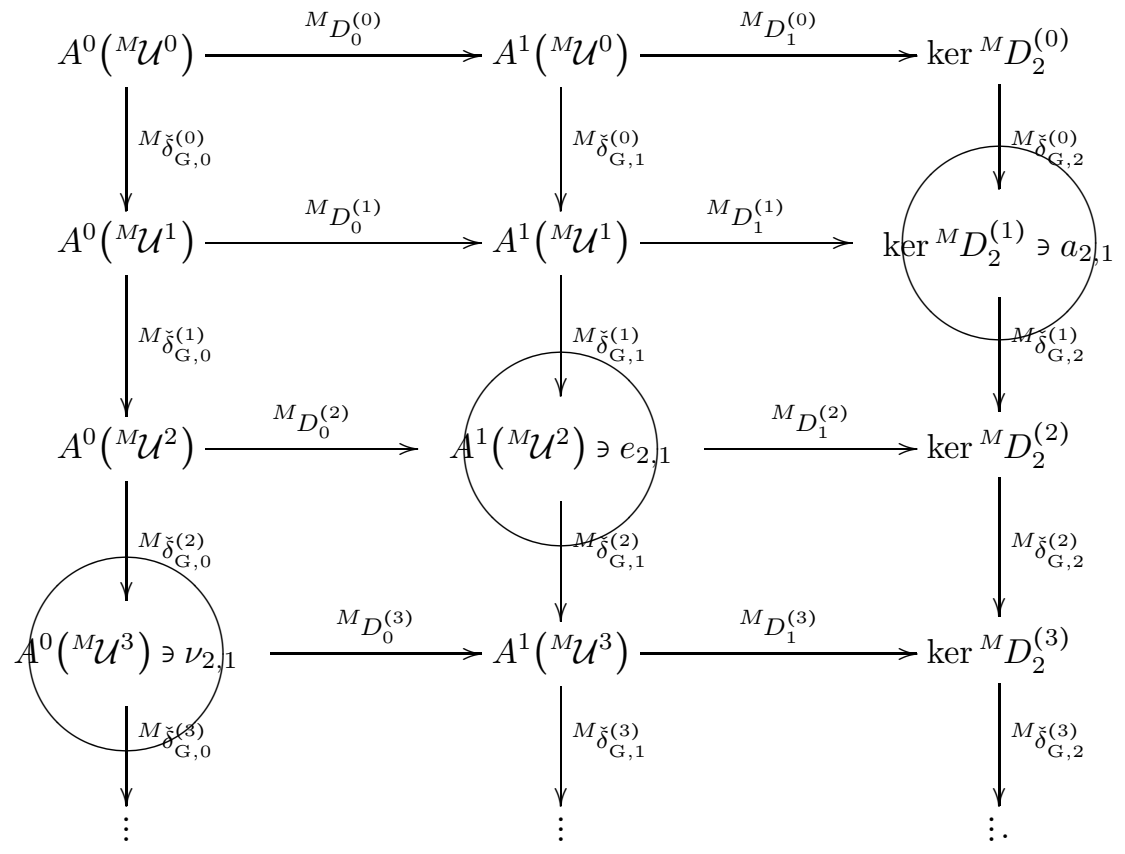

Clearly, its cohomology can be used to classify inequivalent $(\mathrm{G}, \rho)$-structures on a given gerbe $\mathcal{G}$. We readily establish

Proposition 11.10. GSW10, Prop. 6.10] The set of equivalence classes of (G, $\rho)$-equivariant structures on gerbe $\mathcal{G}$ over $M$ is a torsor of the third hypercohomology group $\mathbb{H}^{3}\left({ }^{M} \mathcal{K}\right)$ of the bicomplex ${ }^{M} \mathcal{K}$ of $(11.16)$.

In order to obtain a presentation of the classifying cohomology for $(\mathrm{G}, \rho)$-equivariant structures, we may further map $\mathbb{H}^{3}\left({ }^{M} \mathcal{K}\right)$ to the coset $H^{1}(\mathrm{G} \times M, \mathrm{U}(1)) /{ }^{M} \mathcal{H}^{1,1}$ with respect to the subgroup

$$
{ }^{M} \mathcal{H}^{1,1}:={ }^{M} \check{\delta}_{\mathrm{G}, 2}^{(0)}\left(H^{1}(M, \mathrm{U}(1))\right) .
$$

Properties of this map

$$
\kappa: \mathbb{H}^{3}\left({ }^{M} \mathcal{K}\right) \rightarrow H^{1}(\mathrm{G} \times M, \mathrm{U}(1)) /{ }^{M} \mathcal{H}^{1,1}:[(a, e, \nu)] \mapsto[a]+\mathcal{H}^{1,1},
$$

whose definition is based on the implication

$$
a \mapsto a+{ }^{M} \check{\delta}_{\mathrm{G}, 2}^{(0)} f+{ }^{M} D_{1}^{(1)} l \quad \Rightarrow \quad[a] \mapsto[a]+{ }^{M} \check{\delta}_{\mathrm{G}, 2}^{(0)}[f],
$$

can be obtained through a standard diagram-chasing analysis, performed in Ref. GSW10, to the following effect.

Proposition 11.11. GSW10, Lemmas $6.11 \& 6.12]$ The coset $[a]+\mathcal{H}^{1,1}$ belongs to the image of $\kappa$ iff

(i) ${ }^{M} \check{\delta}_{\mathrm{G}, 2}^{(1)}[a]=0$;

(ii) the image in $H^{3}\left(\pi_{0}(\mathrm{G}), \mathrm{U}(1)^{\pi_{0}(M)}\right)$ under identification ${ }^{M} \zeta_{3}$ of Eq. 11.13) of the class ${ }^{M} \mathscr{B}_{2}^{(1)}([a])$, assigned to $[a]$ by the connecting homomorphism ${ }^{M} \mathscr{B}_{2}^{(1)}$ of Eq. (11.15), is trivial. 
Furthermore, the kernel of $\kappa$ may be naturally identified with the group $H^{2}\left(\pi_{0}(\mathrm{G}), \mathrm{U}(1)^{\pi_{0}(M)}\right)$.

As a simple consequence of the above, we obtain

Corollary 11.12. GSW10, Corollaries $6.12 \& 6.13$ ] If the connected components of G and $M$ are simply connected, then

$$
\mathbb{H}^{3}\left({ }^{M} \mathcal{K}\right) \cong H^{2}\left(\pi_{0}(\mathrm{G}), \mathrm{U}(1)^{\pi_{0}(M)}\right) .
$$

If $\mathrm{G}$ and $M$ are connected, then

$$
\mathbb{H}^{3}\left({ }^{M} \mathcal{K}\right) \cong H^{1}(\mathrm{G}, \mathrm{U}(1)) /\left(r_{m}^{*}\right)_{*} H^{1}(M, \mathrm{U}(1)) \cong Z_{M}^{*},
$$

where $Z_{M}^{*}$ is the group of characters of the kernel $Z_{M}$ of the homomorphism $H_{1}(\mathrm{G}) \rightarrow$ $H_{1}(M)$ induced by the map $r_{m}: \mathrm{G} \rightarrow M: g \mapsto g . m$.

Finally, in the WZW setting, we find

Corollary 11.13. GSW10, Cor.6.14] On the G-space $M:=\mathscr{G}$ in the WZW-model context of Definition 5.1,

$$
\mathbb{H}^{3}\left({ }^{\mathscr{C}} \mathcal{K}\right) \cong H^{1}(\mathrm{G}, \mathrm{U}(1))
$$

11.3. (G, $\lambda$ )-equivariant bi-branes. Having recalled the cohomological description of (G, $\rho$ )equivariant gerbes, as formulated in Ref. [GSW10], we shall discuss bi-branes in a similar vein.

11.3.1. Obstructions to the existence of $\Xi$. Take a 2-cochain $p \in A^{2}\left({ }^{\mathcal{U}}{ }^{0}\right)$ on the worldvolume $Q$ of a $\mathcal{G}$-bi-brane $\mathcal{B}=\left(Q, \iota_{1}, \iota_{2}, \omega, \Phi\right)$, giving local data of a 1 -isomorphism $\Phi$ and hence obeying Eq. (11.2). By virtue of Eqs. (2.41) and (2.42), the curvature of the bi-brane is subject to the identity

$$
{ }^{Q} \check{\delta}_{\mathrm{G}, 3}^{(0)} \bar{\omega}=-\check{\Delta}_{Q, 3}^{(1)} \bar{\rho}+{ }^{Q} D_{2}^{(1)} \bar{\lambda}
$$

where

$$
Q \check{\delta}_{\mathrm{G}, 2}^{(1)} \bar{\lambda}=0 .
$$

We now obtain, using relations (11.2) and (11.4),

$$
{ }^{Q} D_{2}^{(1)}{ }^{Q} \check{\delta}_{\mathrm{G}, 2}^{(0)} p={ }^{Q} \check{\delta}_{\mathrm{G}, 3}^{(0)}{ }^{Q} D_{2}^{(0)} p=\check{\Delta}_{Q, 3}^{(1)}{ }^{M} \check{\delta}_{\mathrm{G}, 3}^{(0)} b+{ }^{Q} \check{\delta}_{\mathrm{G}, 3}^{(0)} \bar{\omega}=-{ }^{Q} D_{2}^{(1)} \check{\Delta}_{Q, 2}^{(1)} a+{ }^{Q} D_{2}^{(1)} \bar{\lambda},
$$

from which we extract the first obstruction to the existence of a $(G, \lambda)$-equivariant structure, namely the class

$$
[D]:=\left[{ }^{Q} \check{\delta}_{\mathrm{G}, 2}^{(0)} p+\check{\Delta}_{Q, 2}^{(1)} a-\bar{\lambda}\right] \in H^{1}(\mathrm{G} \times Q, \mathrm{U}(1))
$$

of the flat line bundle $D \rightarrow \mathrm{G} \times Q$ of Eq. (4.11), with

$$
D \cong\left({ }^{Q} \ell^{*} \Phi \otimes J_{-\lambda}\right) \circ\left(\iota_{1}^{(1) *} \Upsilon^{-1} \otimes \mathrm{id}\right) \circ\left(\Phi_{2^{*}}^{-1} \otimes \mathrm{id}\right) \circ \iota_{2}^{(1) *} \Upsilon .
$$

This class obstructs the existence of a 1-isomorphism $\Xi$ of Eq. (4.14) with local data $s \in$ $A^{1}\left(\mathcal{U}^{1}\right)$ such that relation (11.8) holds. Note that due to Eqs. (11.18) and (11.5),

$$
{ }^{Q} \check{\delta}_{\mathrm{G}, 2}^{(1)}\left({ }^{Q} \check{\delta}_{\mathrm{G}, 2}^{(0)} p+\check{\Delta}_{Q, 2}^{(1)} a-\bar{\lambda}\right)=\check{\Delta}_{Q, 2}^{(2)} M \check{\delta}_{\mathrm{G}, 2}^{(1)} a=-{ }^{Q} D_{1}^{(2)} \check{\Delta}_{Q, 1}^{(2)} e,
$$

hence the isomorphism class $[D]$ is constrained by the relation

$$
H^{1}\left(\mathrm{G}^{2} \times Q, \mathrm{U}(1)\right) \ni Q_{\mathrm{\delta}, 2}^{(1)}[D]=0 .
$$

Let us abbreviate

$$
\operatorname{ker}\left(\mathscr{M}_{\mathrm{G}, 2}^{(1)}: H^{1}(\mathrm{G} \times \mathscr{M}, \mathrm{U}(1)) \rightarrow H^{1}\left(\mathrm{G}^{2} \times \mathscr{M}, \mathrm{U}(1)\right)\right)=: \mathscr{M}_{\mathcal{P}^{1,1}}
$$

Proposition 11.14. Let $a \in A^{2}\left({ }^{M} \mathcal{U}^{1}\right)$ be local data of a 1-isomorphism $\Upsilon:{ }^{M} \ell^{*} \mathcal{G} \rightarrow \mathcal{G}_{2 *} \otimes I_{\rho}$ of a $\mathrm{G}$-equivariant gerbe $(\mathcal{G}, \Upsilon, \gamma ; \rho)$ and let $p \in A^{2}\left({ }^{Q} \mathcal{U}^{0}\right)$ be local data of a 1-isomorphism $\Phi: \iota_{1}^{*} \mathcal{G} \rightarrow \iota_{2}^{*} \mathcal{G} \otimes I_{\omega}$ of $\mathcal{G}$-bi-brane $\left(Q, \iota_{1}, \iota_{2}, \omega, \Phi\right)$. Then, there exists a 2-isomorphism $\Xi:{ }^{Q} \ell^{*} \Phi \stackrel{\cong}{=}\left(\left(\iota_{2}^{(1) *} \Upsilon^{-1} \otimes \mathrm{id}\right) \circ\left(\Phi_{2^{*}} \otimes \mathrm{id}\right) \circ \iota_{1}^{(1) *} \Upsilon\right) \otimes J_{\lambda}$ iff the obstruction class

$$
\left[{ }^{Q} \check{\delta}_{\mathrm{G}, 2}^{(0)} p+\check{\Delta}_{Q, 2}^{(1)} a-\bar{\lambda}\right] \in{ }^{Q} \mathcal{P}^{1,1}
$$

is trivial. 
The cohomology group $H^{1}(\mathrm{G} \times \mathscr{M}, \mathrm{U}(1))$ decomposes naturally as

$$
H^{1}(\mathrm{G} \times \mathscr{M}, \mathrm{U}(1)) \cong H^{1}(\mathrm{G}, \mathrm{U}(1))^{\pi_{0}(\mathscr{M})} \oplus H^{1}(\mathscr{M}, \mathrm{U}(1))^{\pi_{0}(\mathrm{G})}
$$

Taking $\mathscr{M}=Q$, we obtain

Corollary 11.15. If the connected components of $\mathrm{G}$ and $Q$ are 1-connected, there is no obstruction to the existence of a 2-isomorphism $\Xi$.

If $\mathrm{G}$ is connected then it is easy to see (cf. [GSW10, Eq. (6.37)]) that

$$
\mathscr{M}_{\mathcal{P}^{1,1}}=H^{1}(\mathrm{G}, \mathrm{U}(1))^{\pi_{0}(\mathscr{M})}
$$

in terms of the decomposition (11.23). Taking $\mathscr{M}=Q$ again, we infer that

Corollary 11.16. If $\mathrm{G}$ is connected then the obstruction class $[D]$ to the existence of 2-isomorphism $\Xi$ lies in $H^{1}(\mathrm{G}, \mathrm{U}(1))^{\pi_{0}(Q)} \subset H^{1}(\mathrm{G} \times Q, \mathrm{U}(1))$.

It is natural to ask to what extent the obstruction just described can be removed by a change of the equivalence class of the original $(\mathrm{G}, \rho)$-equivariant structure on $\mathcal{G}$, resp. by a change of a representative within this class. In order to answer this question, we study the dependence of $[D]$ on the choice of the class from $\mathbb{H}^{3}\left({ }^{M} \mathcal{K}\right)$ of (local data of) a $(\mathrm{G}, \rho)$ equivariant structure. Note that under a transformation

$$
\begin{gathered}
a \mapsto a+a^{\prime}, \quad c \mapsto e+e^{\prime}, \quad \nu \mapsto \nu+\nu^{\prime} \\
{ }^{M} D_{2}^{(1)} a^{\prime}=0, \quad{ }^{M} D_{1}^{(2)} e^{\prime}=-{ }^{M} \check{\delta}_{\mathrm{G}, 2}^{(1)} a^{\prime}, \quad{ }^{M} D_{0}^{(3)} \nu^{\prime}={ }^{M} \check{\delta}_{\mathrm{G}, 1}^{(2)} e^{\prime}, \quad{ }^{M} \check{\delta}_{\mathrm{G}, 0}^{(3)} \nu^{\prime}=0
\end{gathered}
$$

the obstruction class shifts by

$$
[D] \mapsto[D]+\check{\Delta}_{Q, 2}^{(1)}\left[a^{\prime}\right] .
$$

The constraints imposed upon the triple $\left(a^{\prime}, e^{\prime}, \nu^{\prime}\right)$ identify it as a 3 -cocycle in the hypercohomology of the bicomplex ${ }^{M} \mathcal{K}$ of Eq. (11.16). Denote by $\mathcal{Z}^{3}\left({ }^{M} \mathcal{K}\right)$ the abelian group of all such 3 -cocycles, and by ${ }^{M} \pi^{1,1}$ the canonical projection

$$
\mathcal{Z}^{3}\left({ }^{M} \mathcal{K}\right) \ni\left(a^{\prime}, e^{\prime}, \nu^{\prime}\right) \stackrel{M^{\pi^{1,1}}}{\longmapsto}\left[a^{\prime}\right] \in H^{1}(\mathrm{G} \times M, \mathrm{U}(1))
$$

with the image

$$
{ }^{M} \mathcal{K}^{1,1}:={ }^{M} \pi^{1,1}\left(\mathcal{Z}^{3}\left({ }^{M} \mathcal{K}\right)\right) \subset{ }^{M} \mathcal{P}^{1,1}
$$

We thus arrive at

Corollary 11.17. The component of the class $[D] \in{ }^{Q} \mathcal{P}^{1,1}$, obstructing the existence of 2 isomorphism $\Xi$, that lies in the image of the group ${ }^{M} \mathcal{K}^{1,1}$ of $E q$. (11.27) under the induced cohomology map $\check{\Delta}_{Q, 2}^{(1)}: H^{1}(\mathrm{G} \times M, \mathrm{U}(1)) \rightarrow H^{1}(\mathrm{G} \times Q, \mathrm{U}(1))$ can be removed by a suitable choice of a $(\mathrm{G}, \rho)$-equivariant structure on the gerbe $\mathcal{G}$.

If $\mathrm{G}$ is connected and $M$ is connected, then it is easy to see that $\check{\Delta}_{Q, 2}^{(1)}\left({ }^{M} \mathcal{P}^{1,1}\right)=\{0\}$ (since both $\check{\iota}_{1}^{(1) *}$ and $\check{\iota}_{1}^{(2) *}$ when restricted to $H^{1}(\mathrm{G}, \mathrm{U}(1)) \subset H^{1}(\mathrm{G} \times M, \mathrm{U}(1))$ map as identities into each of the factors in $H^{1}(\mathrm{G}, \mathrm{U}(1))^{\pi_{0}(Q)} \subset H^{1}(\mathrm{G} \times Q, \mathrm{U}(1))$.

Corollary 11.18. If $\mathrm{G}$ and $M$ are connected, then $\check{\Delta}_{Q, 2}^{(1)}\left({ }^{M} \mathcal{K}^{1,1}\right)=\{0\}$.

This applies, in particular, to the case of maximally symmetric non-boundary WZW $\mathcal{G}_{\mathrm{k}}$-bibrane from Definition 5.7 with the (connected) world-volume given by the G-space $Q_{\lambda}=\mathscr{G} \times$ $\mathscr{C}_{\lambda}$ where ${ }^{M} \mathcal{K}^{1,1}={ }^{M} \mathcal{P}^{1,1}=H^{1}(\mathrm{G}, \mathrm{U}(1))$. On the other hand, for the maximally symmetric boundary WZW $\mathcal{G}_{\mathrm{k}}$-bi-brane from Definition 5.3 with the world-volume $\mathscr{C}_{\lambda}$ and $M=\mathscr{G} \sqcup\{\bullet\}$, ${ }^{M} \mathcal{K}^{1,1}={ }^{M} \mathcal{P}^{1,1}=H^{1}(\mathrm{G}, \mathrm{U}(1))^{2}$ and it is mapped by $\check{\Delta}_{Q, 2}^{(1)}$ onto ${ }^{Q} \mathcal{P}^{1,1}=H^{1}(\mathrm{G}, \mathrm{U}(1))$, permitting to remove the obstruction to the existence of a 2 -isomorphism $\Xi$ in this case. If, however, we take a disjoint union of two maximally symmetric boundary WZW $\mathcal{G}_{\mathrm{k}}$-bibranes with $Q=\mathscr{C}_{\lambda_{1}} \sqcup \mathscr{C}_{\lambda_{2}}$ then $\check{\Delta}_{Q, 2}^{(1)}\left({ }^{M} \mathcal{K}^{1,1}\right)$ becomes the diagonal subgroup in ${ }^{Q_{\mathcal{P}}}{ }^{1,1}=$ $H^{1}(\mathrm{G}, \mathrm{U}(1))^{2}$ and one can remove only the diagonal component of the anomaly obstructing the existence of a 2-isomorphism $\Xi$. 
Whenever the first obstruction vanishes, that is whenever a 1-cochain $s \in A^{1}\left({ }^{Q} \mathcal{U}^{1}\right)$ can be found (for $\left\{{ }^{2} \mathcal{U}^{1}\right\}$ sufficiently fine) for which identity (11.8) is satisfied, we may enquire whether the 2-isomorphism $\Xi$ with local data given by $s$ obeys constraints (8.8). In general, we only find

$$
{ }^{Q} D_{1}^{(2)} Q \check{\delta}_{\mathrm{G}, 1}^{(1)} s={ }^{Q} \check{\delta}_{\mathrm{G}, 2}^{(1)}{ }^{Q} D_{1}^{(1)} s=-{ }^{Q} \check{\delta}_{\mathrm{G}, 2}^{(1)} \check{\Delta}_{Q, 2}^{(1)} a=-\check{\Delta}_{Q, 2}^{(2)} M \check{\delta}_{\mathrm{G}, 2}^{(1)} a=\check{\Delta}_{Q, 2}^{(2)}{ }^{M} D_{1}^{(2)} e={ }^{Q} D_{1}^{(2)} \check{\Delta}_{Q, 1}^{(2)} e,
$$

and so we end up with a class

$$
[\sigma]:=\left[{ }^{Q} \check{\delta}_{\mathrm{G}, 1}^{(1)} s-\check{\Delta}_{Q, 1}^{(2)} e\right] \in H^{0}\left(\mathrm{G}^{2} \times Q, \mathrm{U}(1)\right)
$$

that may be nontrivial. Note that

$$
{ }^{Q} \check{\delta}_{\mathrm{G}, 1}^{(2)}[\sigma]=\left[-{ }^{Q} \check{\delta}_{\mathrm{G}, 1}^{(2)} \check{\Delta}_{Q, 1}^{(2)} e\right]=-\left[\check{\Delta}_{Q, 1}^{(3)} \check{\check{\delta}}_{\mathrm{G}, 1}^{(2)} e\right]=-\left[{ }^{Q} D_{0}^{(3)} \check{\Delta}_{Q, 0}^{(3)} \nu\right]=0
$$

whence

$$
[\sigma] \in \operatorname{ker}\left({ }^{Q} \check{\delta}_{\mathrm{G}, 1}^{(2)}: H^{0}\left(\mathrm{G}^{2} \times Q, \mathrm{U}(1)\right) \rightarrow H^{0}\left(\mathrm{G}^{3} \times Q, \mathrm{U}(1)\right)\right)=:{ }^{Q} \mathcal{P}^{0,2} .
$$

The actual obstruction to the commutativity of diagram (H.4) belongs to a coset of the above group that can be identified by exploiting, similarly as for the gerbe, the intrinsic ambiguity in the definition of the 1-cochain $s$. First, the latter may be shifted as

$$
s \mapsto s+s^{\prime \prime}, \quad s^{\prime \prime} \in \operatorname{ker}^{Q} D_{1}^{(1)},
$$

whereby $[\sigma]$ undergoes a transformation $[\sigma] \mapsto[\sigma]+{ }^{Q} \check{\delta}_{\mathrm{G}, 1}^{(1)}\left[s^{\prime \prime}\right]$. Write

$$
{ }^{Q} \mathcal{H}^{0,2}:={ }^{Q} \check{\delta}_{\mathrm{G}, 1}^{(1)}\left(H^{0}(\mathrm{G} \times Q, \mathrm{U}(1))\right)
$$

The upshot of our analysis is the following

Proposition 11.19. Let $e \in A^{1}\left({ }^{M} \mathcal{U}^{2}\right)$ be local data of a 2-isomorphism $\gamma$ of a $(\mathrm{G}, \rho)$-equivariant structure on a gerbe $\mathcal{G}$ over $M$, and let $s \in A^{1}\left({ }^{Q} \mathcal{U}^{1}\right)$ be local data of a 2-isomorphism $\Xi:{ }^{2} \ell^{*} \Phi \stackrel{\cong}{\Longrightarrow}\left(\left(\iota_{2}^{(1) *} \Upsilon^{-1} \otimes \mathrm{id}\right) \circ\left(\Phi_{2^{*}} \otimes \mathrm{id}\right) \circ \iota_{1}^{(1) *} \Upsilon\right) \otimes J_{\lambda}$. The 2-isomorphism $\Xi$ may be chosen so that it satisfies the coherence condition of Eq. (8.8) iff the obstruction class

$$
\left[{ }^{Q} \check{\delta}_{\mathrm{G}, 1}^{(1)} s-\check{\Delta}_{Q, 1}^{(2)} e\right]+{ }^{Q} \mathcal{H}^{0,2} \in{ }^{Q} \mathcal{P}^{0,2} /{ }^{Q} \mathcal{H}^{0,2}
$$

is trivial.

It is to be noted that the obstruction identified in the previous proposition can be further reduced by exploiting the residual freedom of choice of a $(\mathrm{G}, \rho)$-equivariant structure on $\mathcal{G}$. Indeed, consider an admissible transformation (11.25) of local data of a $(\mathrm{G}, \rho)$-equivariant structure on $\mathcal{G}$, accompanied by a shift

$$
s \mapsto s+s^{\prime}, \quad \quad{ }^{Q} D_{1}^{(1)} s^{\prime}=-\check{\Delta}_{Q, 2}^{(1)} a^{\prime}, \quad s^{\prime} \in A^{1}\left({ }^{Q} \mathcal{U}^{1}\right),
$$

i.e. altogether constrained so that they do not spoil the anomaly cancellations ensured previously. The resultant shift of $[\sigma]$ is given by

$$
[\sigma] \mapsto[\sigma]+\left[{ }^{Q} \check{\delta}_{\mathrm{G}, 1}^{(1)} s^{\prime}-\check{\Delta}_{Q, 1}^{(2)} e^{\prime}\right]
$$

and the intrinsic ambiguity of the 1-cochain $s^{\prime}$ captured by Eq. (11.31) leads us, once more, to consider the coset $\left[{ }^{Q} \check{\delta}_{\mathrm{G}, 1}^{(1)} s^{\prime}-\check{\Delta}_{Q, 1}^{(2)} e^{\prime}\right]+{ }^{Q} \mathcal{H}^{0,2}$ rather than the class itself. In order to properly account for the observed freedom of readjustment of the structure under construction, we consider the tricomplex ${ }^{(M, Q) \mathcal{K}}$ formed by the pair of bicomplexes ${ }^{M} \mathcal{K}$ and ${ }^{Q} \mathcal{K}$, cf. 
Eq. (11.16),

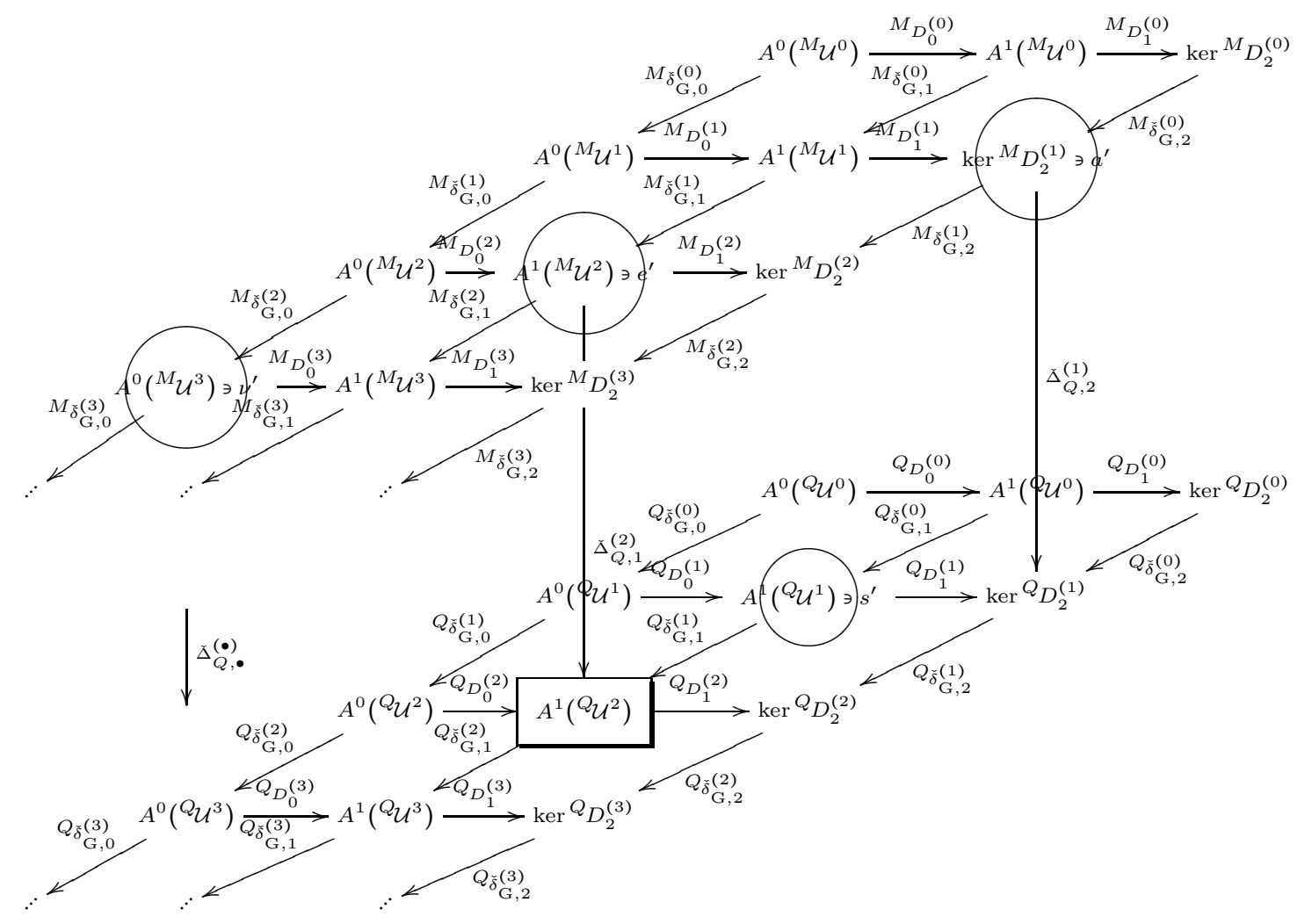

using the family $\left\{\check{\Delta}_{Q, p}^{(q)}\right\}$ of chain maps, and with the following assignment of the total degree

$$
\operatorname{deg} A^{p}\left({ }^{M} \mathcal{U}^{q}\right)=p+q, \quad \operatorname{deg} A^{p}\left({ }^{Q} \mathcal{U}^{q}\right)=p+q+1
$$

Our discussion points to the rôle of a group homomorphism

$$
\operatorname{ker}\left(\check{\Delta}_{Q, 2}^{(1)} \circ{ }^{M} \pi^{1,1}\right) \ni\left(a^{\prime}, e^{\prime}, \nu^{\prime}\right) \stackrel{\mathscr{H}}{\longmapsto}\left[{ }^{Q} \check{\delta}_{\mathrm{G}, 1}^{(1)} s^{\prime}-\check{\Delta}_{Q, 1}^{(2)} e^{\prime}\right]+{ }^{Q} \mathcal{H}^{0,2} \in{ }^{Q} \mathcal{P}^{0,2} /{ }^{Q} \mathcal{H}^{0,2} .
$$

We are thus led to the following

Corollary 11.20. The component from the image of map $\mathscr{H}$ of Eq. (11.33) of the class $\left[{ }^{Q} \check{\delta}_{\mathrm{G}, 1}^{(1)} s-\check{\Delta}_{Q, 1}^{(2)} e\right] \in{ }^{Q} \mathcal{P}^{0,2} /{ }^{Q} \mathcal{H}^{0,2}$ obstructing the existence of a coherent 2-isomorphism $\Xi$ of Eq. (4.14) (and defined in terms of local data $s \in A^{1}\left({ }^{Q} \mathcal{U}^{1}\right)$ of $\Xi$ and local data $e \in A^{1}\left({ }^{M} \mathcal{U}^{2}\right)$ of a 2-isomorphism $\gamma$ of Eq. (8.2)) can be removed by a suitable choice of a $(\mathrm{G}, \rho)$-equivariant structure on gerbe $\mathcal{G}$, and a choice of a 2-isomorphism $\Xi$ compatible with the former in the sense of Eq. (11.32).

At this stage, we may employ identification (11.13) (for $\mathrm{GQ}$ ) to reformulate the description of the latter obstruction to the existence of a coherent $(\mathrm{G}, \lambda)$-equivariant structure on $\mathcal{B}$ in more tractable terms. Noting that identity $(11.29)$ translates into the statement

$$
{ }^{Q} \zeta_{2}([\sigma]) \in \operatorname{ker} \delta_{\pi_{0}(\mathrm{G})}^{(2)}
$$

we arrive at

Corollary 11.21. The image ${ }^{Q} \zeta_{2}\left(\left[{ }^{Q} \check{\delta}_{\mathrm{G}, 1}^{(1)} s-\check{\Delta}_{Q, 1}^{(2)} e\right]\right)=:{ }^{Q} u^{2}$ of the obstruction class $\left[{ }^{Q} \check{\delta}_{\mathrm{G}, 1}^{(1)} s-\right.$ $\left.\check{\Delta}_{Q, 1}^{(2)} e\right]$ for a coherent 2-isomorphism $\Xi$ under the identification of $E q$. (11.13) is a $\mathrm{U}(1)^{\pi_{0}(Q)}$. valued 2 -cocycle of the group $\pi_{0}(\mathrm{G})$, and that of the obstruction coset $\left[{ }^{Q} \check{\delta}_{\mathrm{G}, 1}^{(1)} s-\check{\Delta}_{Q, 1}^{(2)} e\right]+{ }^{Q} \mathcal{H}^{0,2}$ is the cohomology class $\left[{ }^{Q} u^{2}\right] \in H^{2}\left(\pi_{0}(\mathrm{G}), \mathrm{U}(1)^{\pi_{0}(Q)}\right)$.

Eq. 11.14) now yields

Corollary 11.22. If the symmetry group $\mathrm{G}$ is connected and a 2-isomorphism $\Xi$ exists, then it can always be chosen in a coherent form. 
Clearly, this applies to the maximally symmetric WZW $\mathcal{G}_{\mathrm{k}}$-bi-branes from Definitions 5.3 and 5.7 in Sections 5.2 and 5.3 .

11.3.2. Classification. Let us now examine equivalences among $(\mathrm{G}, \lambda)$-equivariant structures on a given $\mathcal{G}$-bi-brane $\mathcal{B}=\left(Q, \iota_{1}, \iota_{2}, \omega, \Phi\right)$ in detail. Suppose that there exist two such structures, $\left(\mathcal{B}, \Xi_{\beta} ; \kappa\right), \beta=1,2$ on $\mathcal{B}$. Denote local data of the respective 2-isomorphisms as $s_{\beta}$ and $\theta_{\beta}$ (the coherence 0 -cochain), and their differences as

$$
s_{2,1}:=s_{2}-s_{1}, \quad \theta_{2,1}:=\theta_{2}-\theta_{1} .
$$

For a fixed $(\mathrm{G}, \rho)$-equivariant structure on $\mathcal{G}$, we have

$$
{ }^{Q} D_{1}^{(1)} s_{\beta}=-{ }^{Q} \check{\delta}_{\mathrm{G}, 2}^{(0)} p-\check{\Delta}_{Q, 2}^{(1)} a+\bar{\lambda}, \quad \quad{ }^{Q} D_{0}^{(2)} \theta_{\beta}={ }^{Q} \check{\delta}_{\mathrm{G}, 1}^{(1)} s_{\beta}-\check{\Delta}_{Q, 1}^{(2)} e,
$$

and so the difference 1-cochain $s_{2,1}$ automatically satisfies the identities

$$
{ }^{Q} D_{1}^{(1)} s_{2,1}=0, \quad \quad{ }^{Q} \check{\delta}_{\mathrm{G}, 1}^{(1)} s_{2,1}={ }^{Q} D_{0}^{(2)} \theta_{2,1},
$$

from which it follows that it defines a class

$$
\left[s_{2,1}\right] \in H^{0}(\mathrm{G} \times Q, \mathrm{U}(1))
$$

with the property

$$
Q_{\check{\delta}_{\mathrm{G}, 1}^{(1)}}\left[s_{2,1}\right]=0 .
$$

According to Definition 8.5, an equivalence between the two structures, whenever it exists, can be expressed in purely cohomological terms with the help of local data $t \in A^{1}\left(\mathcal{U}^{0}\right)$ and $\tau \in A^{0}\left(\mathcal{U}^{1}\right)$ of a 2 -isomorphism $\psi: \Phi \Rightarrow \Phi$ subject to the additional coherence constraint tantamount to Eq. (8.9),

$$
\begin{array}{ll}
s_{2,1}={ }^{Q} \check{\delta}_{\mathrm{G}, 1}^{(0)} t+{ }^{Q} D_{0}^{(1)} \tau, & { }^{Q} D_{1}^{(0)} t=0, \\
\theta_{2,1}={ }^{Q} \check{\delta}_{\mathrm{G}, 0}^{(1)} \tau, &
\end{array}
$$

where the last relation follows from Eqs. (11.34) and (11.35). The above equations hold iff

$$
\left[s_{2,1}\right]={ }^{Q} \check{\delta}_{\mathrm{G}, 1}^{(0)}[t] .
$$

As a result, we obtain, using identification (11.13),

Proposition 11.23. The set of equivalence classes of $(\mathrm{G}, \lambda)$-equivariant $\mathcal{G}$-bi-branes with world-volume $Q$ is, for a fixed $(\mathrm{G}, \rho)$-equivariant structure on a gerbe $\mathcal{G}$, a torsor of the first cohomology group $H^{1}\left(\pi_{0}(\mathrm{G}), \mathrm{U}(1)^{\pi_{0}(Q)}\right)$ of the group $\pi_{0}(\mathrm{G})$ with values in the $\pi_{0}(\mathrm{G})$ module $\mathrm{U}(1)^{\pi_{0}(Q)}$.

In particular,

Corollary 11.24. If the symmetry group $\mathrm{G}$ is connected, a $(\mathrm{G}, \lambda)$-equivariant structure on $a \mathcal{G}$-bi-brane, whenever it exists, is unique up to an equivalence.

This is the case for the maximally symmetric WZW $\mathcal{G}_{k}$-bi-branes from Sections 5.2 and 5.3 .

11.4. G-equivariant inter-bi-branes. The completion of the construction of a full-blown $(\mathrm{G}, \rho, \lambda)$-equivariant string background requires one further step that consists in checking the compatibility of the structures, introduced hitherto as an extension of gerbe and bi-brane data, with the structure of an inter-bi-brane. We address this final issue below.

11.4.1. Obstructions. Given local data $h_{n} \in A^{1}\left({ }^{T_{n}} \mathcal{U}^{0}\right)$, constrained by identity (11.3), of 2isomorphism $\varphi_{n}$ of a $(\mathcal{G}, \mathcal{B})$-inter-bi-brane $\left(T_{n}\left(\varepsilon_{n}^{k, k+1}, \pi_{n}^{k, k+1}\right) ; \varphi_{n}\right)$ with component worldvolumes $T_{n}$, we readily compute

$$
\begin{aligned}
{ }^{T_{n}} D_{1}^{(1) T_{n} \check{\delta}_{\mathrm{G}, 1}^{(0)} h_{n}} & ={ }^{T_{n}} \check{\delta}_{\mathrm{G}, 2}^{(0) T_{n}} D_{1}^{(0)} h_{n}={ }^{T_{n}} \check{\delta}_{\mathrm{G}, 2}^{(0)} \check{\Delta}_{T_{n}, 2}^{(0)} p=-\check{\Delta}_{T_{n}, 2}^{(1)} \check{\delta}_{\mathrm{G}, 2}^{(0)} p \\
& =\check{\Delta}_{T_{n}, 2}^{(1)}{ }^{Q} D_{1}^{(1)} s+\check{\Delta}_{T_{n}, 2}^{(1)} \check{\Delta}_{Q, 2}^{(1)} a-\check{\Delta}_{T_{n}, 2}^{(1)} \bar{\lambda}={ }^{T_{n}} D_{1}^{(1)} \check{\Delta}_{T_{n}, 1}^{(1)} s .
\end{aligned}
$$

This gives us a class

$$
d_{n}:=\left[{ }^{T_{n}} \check{\delta}_{\mathrm{G}, 1}^{(0)} h_{n}-\check{\Delta}_{T_{n}, 1}^{(1)} s\right] \in H^{0}\left(\mathrm{G} \times T_{n}, \mathrm{U}(1)\right)
$$


that obstructs a coherent extension of a $(\mathrm{G}, \rho, \lambda)$-equivariant structure on the gerbe $\mathcal{G}$ and the bi-brane $\mathcal{B}$ (whose existence we are assuming) to the complete background. Viewed as a locally constant $\mathrm{U}(1)$-valued function on $\mathrm{G} \times T_{n}, d_{n}$ coincides with the function defined by equality (4.17). In this manner, we establish

Proposition 11.25. Let $s \in A^{1}\left(\mathcal{Q}^{1}\right)$ be local data of a 2-isomorphism $\Xi$ of the $(\mathrm{G}, \lambda)$ equivariant $\mathcal{G}$-bi-brane $(\mathcal{B}, \Xi ; \kappa)$, and let $h_{n} \in A^{1}\left({ }^{T_{n}} \mathcal{U}^{0}\right)$ be local data of the 2-isomorphism $\varphi_{n}$ of (2.2) of the $(\mathcal{G}, \mathcal{B})$-inter-bi-brane $\mathcal{J}_{n}$. Then, a $(\mathrm{G}, \rho)$-equivariant structure on $\mathcal{G}$ together with a $(\mathrm{G}, \lambda)$-equivariant structure on $\mathcal{B}$ compatible with it extend coherently to a $(\mathrm{G}, \rho, \lambda)$-equivariant structure on $\left(\mathcal{G}, \mathcal{B}, \mathcal{J}_{n}\right)$, as expressed by Definition 8.6, iff the obstruction class

$$
d_{n} \in H^{0}\left(\mathrm{G} \times T_{n}, \mathrm{U}(1)\right)
$$

of (11.36) is trivial.

We have

$$
T_{n} \check{\delta}_{\mathrm{G}, 1}^{(1)} d_{n}=\left[-\check{\Delta}_{T_{n}, 1}^{(2)} Q \check{\delta}_{\mathrm{G}, 1}^{(1)} s\right]=\left[-\check{\Delta}_{T_{n}, 1}^{(2)} \check{\Delta}_{Q, 1}^{(2)} e-{ }^{T_{n}} D_{0}^{(2)} \check{\Delta}_{T_{n}, 0}^{(2)} \theta\right]=0,
$$

i.e.

$$
d_{n} \in \operatorname{ker}\left({ }^{T_{n}} \check{\delta}_{\mathrm{G}, 1}^{(1)}: H^{0}\left(\mathrm{G} \times T_{n}, \mathrm{U}(1)\right) \rightarrow H^{0}\left(\mathrm{G}^{2} \times T_{n}, \mathrm{U}(1)\right)\right)=:{ }^{T_{n}} \mathcal{P}^{0,1} .
$$

The identification (11.13) permits, as before, to reformulate the description of the obstruction in the language of the cohomology of the group $\pi_{0}(\mathrm{G})$, this time with values in $\mathrm{U}(1)^{\pi_{0}\left(T_{n}\right)}$. We have

Corollary 11.26. The image ${ }^{T_{n}} \zeta_{1}\left(\left[d_{n}\right]\right)=:^{T_{n}} u^{1}$, under the identification of Eq. (11.13), of the obstruction class $d_{n}$ of (11.36) for a coherent extension of a $(\mathrm{G}, \rho)$-equivariant structure on the gerbe $\mathcal{G}$ together with a $(\mathrm{G}, \lambda)$-equivariant structure on the $\mathcal{G}$-bi-brane $\mathcal{B}$ compatible with it to a $(\mathrm{G}, \rho, \lambda)$-equivariant structure on the background $\left(\mathcal{G}, \mathcal{B}, \mathcal{J}_{n}\right)$ is a $\mathrm{U}(1)^{\pi_{0}\left(T_{n}\right)}$ valued 1-cocycle of the group $\pi_{0}(\mathrm{G})$.

Since 1-cocycles on the trivial group $\mathbf{1}$ are trivial, we obtain

Corollary 11.27. For a connected group $\mathrm{G}$, the obstruction classes $d_{n}$ are trivial.

This applies, in particular, to the maximally symmetric WZW $\mathcal{G}_{\mathrm{k}}$-bi-branes and inter-bibranes from Sections 5.3 and 5.4 .

In the general case, pursuing the logic of the preceding sections, we admit the possibility of using the freedom of choice, consistent with previous stages of the construction, of a $(\mathrm{G}, \rho)$ equivariant structure on $\mathcal{G}$ and of a $(\mathrm{G}, \lambda)$-equivariant structure on $\mathcal{B}$ compatible with it to rid the string background $\left(\mathcal{G}, \mathcal{B}, \mathcal{J}_{n}\right)$ of at least a part of the obstruction to the existence of a full-blown coherent $(\mathrm{G}, \rho, \lambda)$-equivariant structure. Thus, we assume Eqs. (11.4)-(11.9) to be obeyed by a collection of data $(a, e, \nu ; s, \theta)$ introduced previously, and examine the effect on $d_{n}$ of the most general transformation of the initial data,

$$
(a, e, \nu ; s, \theta) \longmapsto(a, e, \nu ; s, \theta)+\left(a^{\prime}, e^{\prime}, \nu^{\prime} ; s^{\prime}, \theta^{\prime}\right),
$$

that preserves the structure of the cohomological relations (11.4)-(11.9). Components of such a transformation are constrained to satisfy a 'homogeneous' variant of those relations, i.e.

$$
\begin{aligned}
{ }^{M} D_{2}^{(1)} a^{\prime}=0, & { }^{M} D_{1}^{(2)} e^{\prime}=-{ }^{M} \check{\delta}_{\mathrm{G}, 2}^{(1)} a^{\prime}, & { }^{M} D_{0}^{(3)} \nu^{\prime}={ }^{M} \check{\delta}_{\mathrm{G}, 1}^{(2)} e^{\prime}, \\
{ }^{Q} D_{1}^{(1)} s^{\prime}=-\check{\Delta}_{Q, 2}^{(1)} a^{\prime}, & { }^{Q} D_{0}^{(2)} \theta^{\prime}={ }^{Q} \check{\delta}_{\mathrm{G}, 1}^{(1)} s^{\prime}-\check{\Delta}_{Q, 1}^{(2)} e^{\prime}, & Q \check{\delta}_{\mathrm{G}, 0}^{(2)} \theta^{\prime}=-\check{\Delta}_{Q, 0}^{(3)} \nu^{\prime} .
\end{aligned}
$$

This identifies $\left(a^{\prime}, e^{\prime}, \nu^{\prime} ; 0, s^{\prime}, \theta^{\prime}\right)$ as a 3 -cocycle of the hypercohomology of the tricomplex $(M, Q) \mathcal{K}$ from the kernel of the canonical projection

$$
\mathcal{Z}^{3}\left({ }^{(M, Q)} \mathcal{K}\right) \ni\left(a^{\prime}, e^{\prime}, \nu^{\prime} ; x, s^{\prime}, \theta^{\prime}\right) \stackrel{(M, Q)_{\pi^{1,0}}}{\longmapsto}[x] \in H^{1}(Q, \mathrm{U}(1))
$$

\footnotetext{
${ }^{7}$ We implicitly assume that the metric on $M$ has the desired invariance properties.
} 
defined on the abelian group $\mathcal{Z}^{3}\left({ }^{(M, Q)} \mathcal{K}\right)$ of 3-cocycles of the hypercohomology of the tricomplex ${ }^{(M, Q)} \mathcal{K}$. On this subspace, there exists a well-defined group homomorphism

$$
\operatorname{ker}\left({ }^{(M, Q)} \pi^{1,0}\right) \ni\left(a^{\prime}, e^{\prime}, \nu^{\prime} ; 0, s^{\prime}, \theta^{\prime}\right) \stackrel{\mathscr{K}}{\longmapsto}\left[\check{\Delta}_{T_{n}, 1}^{(1)} s^{\prime}\right] \in{ }^{T_{n}} \mathcal{P}^{0,1}
$$

and we have

Corollary 11.28. The component of the obstruction class $d_{n}$ from the image of map $\mathscr{K}$ of $E q$. (11.37) may be removed by a suitable choice of a $(\mathrm{G}, \rho)$-equivariant structure on $\mathcal{G}$, and that of a $(\mathrm{G}, \lambda)$-equivariant structure on $\mathcal{B}$.

Let us close this Section by summarizing the situation for the WZW $\sigma$-model.

Corollary 11.29. In the $W Z W$-model context of Definition 5.1 with the maximally symmetric $\mathcal{G}_{\mathrm{k}}$-bi-branes and inter-bi-branes discussed in Section 1 , the only obstructions to the existence of a $\mathrm{G}$-equivariant structure on the corresponding string background come from the global gauge anomalies for topologically trivial gauge fields that were discussed in Section S. In their absence, the set of equivalence classes of G-equivariant structures forms a torsor under the group $H^{1}(\mathrm{G}, \mathrm{U}(1))$.

Remark 11.30. The freedom of choice of a G-equivariant structure appears in the WZWmodel gauged Feynman amplitudes in topologically nontrivial sectors with gauge-fields given by connections in nontrivial principal G-bundles classified by $\pi_{1}(\mathrm{G})$. A change of the equivariant structure by an element of $H^{1}(\mathrm{G}, \mathrm{U}(1))$ that may be viewed as a character of $\pi_{1}(\mathrm{G})$ multiplies the amplitude by the corresponding value of the character. This was discussed in detail in Ref. [GSW10] for the defect-free amplitudes and the discussion extends to the case with defects.

\section{Conclusions}

We have presented a detailed study of the gauging of rigid symmetries of the two-dimensional bosonic $\sigma$-model on world-sheets $\Sigma$ with defects forming a graph $\Gamma$ embedded in the worldsheet, including the case of boundary defects. Classical fields mapping $\Sigma \backslash \Gamma$ into the target space of the model may jump across the defect lines and at defect junctions. The jumps across the lines of the graph are described by maps from the lines into bi-brane world-volumes and the jumps at the line junctions by maps from the junction points to inter-bi-brane worldvolumes. The Wess-Zumino contributions to the action of the $\sigma$-model are given in terms of a holonomy of a gerbe on the target manifold supplemented with bi-branes and inter-bibranes equipped with a gerbish structure formulated in terms of the 2-category of gerbes with connections. The rigid symmetries of the model have been identified with group actions on the $\sigma$-model target space (including the bi-brane and inter-bi-brane world-volumes) that respect the target-space metric and the gerbish structure in a way that ensures the invariance of the $\sigma$-model Feynman amplitudes. Promoting the rigid symmetries to the rank of local ones required coupling the model to the world-sheet gauge fields of the rigid-symmetry group G. For a gauge field given by a connection in a trivial principal G-bundle over $\Sigma$, the appropriate gauging procedure that renders the gauged amplitudes invariant under infinitesimal gauge transformation has been defined as a slight extension of that introduced in Refs. JJJMO90, HS91 for bulk amplitudes and in Ref. FOM05 for the boundary ones. Various interpretations of the conditions required by such a construction have been presented. We have analysed the invariance of the corresponding gauged amplitudes under large gauge transformations nonhomotopic to the identity, identifying the defect- and boundary-contributions to the global gauge anomalies. These results have been illustrated on the example of the WZW amplitudes, extending the work of Ref. [GSW10] where this was done for the defect-free models. We have subsequently extended the notion of equivariant gerbes introduced in Ref. GSW10 to include equivariant structures on bi-branes and inter-bi-branes, and showed that such structures permit a natural coupling of the $\sigma$-model amplitudes with defects to world-sheet gauge fields given by connections in arbitrary principal G-bundles in a way that assures the invariance of the resulting amplitudes under all gauge transformations, including the large ones. Obstructions to the existence and the classification of such equivariant structures have been fully analysed. In particular, we have showed that in the context of WZW models with 
maximally symmetric defects, all obstructions manifest themselves already in the presence of topologically trivial gauge fields, similarly as in the defect-free case. In summary, the present paper provides a realisation of a major step in the study of global gauge anomalies in two-dimensional bosonic $\sigma$-models with Wess-Zumino terms in the action, initiated in Ref. GSW10. 


\section{Appendix A. A proof of the "Only if" part of Proposition 2.24}

That conditions (2.26) and (2.27) are necessary for the infinitesimal rigid invariance of Feynman amplitudes was noticed already in Ref. GSW10 where field configurations without defects were considered. It is straightforward to see in that case that the vanishing of the right-hand side of Eq. 2.25) for arbitrary world-sheet metrics $\gamma$ and arbitrary $\varphi: \Sigma \rightarrow M$ requires that condition $(2.26)$ be satisfied. Using subsequently the fact that the homology group $H_{2}(M)$ is generated by singular 2-cycles $c_{2}^{M}(\varphi)$ obtained from fields $\varphi$ and triangulations of $\Sigma$, one infers condition (2.27).

Next, let us consider world-sheets $\Sigma$ with defect quivers $\Gamma$ without defect junctions, i.e. composed of closed loops only. Given a triangulation of $\Sigma$ compatible with $\Gamma$, each networkfield configuration $(\varphi \mid \Gamma)$ gives rise to a singular 1-cycle $c_{1}^{Q}(\varphi)$ in $Q$ and a singular 2-chain $c_{2}^{M}(\varphi)$ in $M$. The 1-cycle $c_{1}^{Q}(\varphi)$ is obtained from $\left.\varphi\right|_{\Gamma}$ and the induced triangulation of $\Gamma$, and the 2-chain $c_{2}^{M}(\varphi)$ from $\left.\varphi\right|_{\Sigma \backslash \Gamma}$ extended by the boundary values and the triangulation of $\Sigma$. Let

$$
\Delta^{Q}=\left(\iota_{2}\right)_{*}-\left(\iota_{1}\right)_{*}
$$

be the combination of maps on singular chains induced by the $\iota_{\alpha}: Q \rightarrow M$. The defining requirements for a network-field configuration imply that

$$
\Delta^{Q} c_{1}^{Q}(\varphi)=-\partial c_{2}^{M}(\varphi) .
$$

Note that this means that the homology class $\left[c_{1}^{Q}(\varphi)\right] \in H_{1}(Q)$ belongs to the kernel ker $\Delta^{Q}$ of the induced map $\Delta^{Q}: H_{1}(Q) \rightarrow H_{1}(M)$. It is not difficult to see that $\operatorname{ker} \Delta^{Q}$ may be generated this way. Now, assuming that Eq. (2.27) holds, we have

$$
\begin{aligned}
\int_{\Sigma} \varphi^{*}\left(\iota_{a} \mathrm{H}\right)+\int_{\Gamma} \varphi^{*}\left(\iota_{a} \omega\right)=\int_{c_{2}^{M}(\varphi)} \iota_{a} \mathrm{H}+\int_{c_{1}^{Q}(\varphi)} \iota_{a} \omega & =-\int_{\partial c_{2}^{M}(\varphi)} \kappa_{a}+\int_{c_{1}^{Q}(\varphi)} \iota_{a} \omega \\
& =\int_{\Delta_{c_{1}^{Q}(\varphi)} \kappa_{a}+\int_{c_{1}^{Q}(\varphi)} \iota_{a} \omega}=\int_{c_{1}^{Q}(\varphi)}\left(\iota_{a} \omega+\Delta_{Q} \kappa_{a}\right) .
\end{aligned}
$$

The 1-forms $\iota_{a} \omega+\Delta_{Q} \kappa_{a}$ are closed due to relations (2.3) and (2.27). We want to show that the vanishing of the right-hand side of Eq. (A.2) for all $c_{1}^{Q}(\varphi)$ implies that for an appropriate choice of 1-forms $\kappa_{a}$ on $M$, fixed by Eq. (2.27) up to addition of closed 1-forms $\kappa_{a}^{\prime}$, the induced cohomology classes $\left[\iota_{a} \omega+\Delta_{Q} \kappa_{a}\right] \in H^{1}(Q, \mathbb{R})$ vanish, whence relation (2.28) ensues. This follows from the cohomology exact sequence

$$
H^{1}(M, \mathbb{R}) \stackrel{\Delta_{Q}}{\longrightarrow} H^{1}(Q, \mathbb{R}) \longrightarrow \operatorname{Hom}_{\mathbb{Z}}\left(\operatorname{ker} \Delta^{Q}, \mathbb{R}\right)
$$

obtained by applying the exact functor $\operatorname{Hom}_{\mathbb{Z}}(\cdot, \mathbb{R})$ to the homology exact sequence

$$
\operatorname{ker} \Delta^{Q} \hookrightarrow H_{1}(Q) \stackrel{\Delta^{Q}}{\longrightarrow} H_{1}(M) .
$$

Finally, consider closed world-sheets $\Sigma$ with general defect quivers $\Gamma$. A network-field configuration $(\varphi \mid \Gamma)$ together with a triangulation of $\Sigma$ compatible with $\Gamma$ gives rise to a singular 0-cycle $c_{0}^{T}(\varphi)$ in $T$, to a singular 1-chain $c_{1}^{Q}(\varphi)$ in $Q$ and to a singular 2-chain $c_{2}^{M}(\varphi)$ in $M$,

$$
c_{0}^{T}(\varphi)=\sum_{j \in \mathfrak{V}_{\Gamma}} \pm \varphi(j),
$$

where the + sign is taken for the positive defect junctions (i.e. the ones with the counterclockwise ordering of defect lines) and the - sign for the negative junctions (that have defect lines ordered clockwise). The 1-chain $c_{1}^{Q}(\varphi)$ results from extending $\left.\varphi\right|_{\Gamma \backslash \mathfrak{V}_{\Gamma}}$ by the boundary values, and the 2 -chain $c_{2}^{M}(\varphi)$ is obtained as before. Let

$$
\Delta^{T_{n}}=\sum_{k=1}^{n} \varepsilon_{n}^{k, k+1}\left(\pi_{n}^{k, k+1}\right)_{*}
$$

be the combination of maps on singular chains induced by the $\pi_{n}^{k, k+1}: T_{n} \rightarrow Q$. Note that $\Delta^{Q} \circ \Delta^{T_{n}}=0$. Let $\Delta^{T}=\sum_{n \geq 3} \Delta^{T_{n}}$. One has

$$
\Delta^{T} c_{0}^{T}(\varphi)=\partial c_{1}^{Q}(\varphi), \quad \Delta^{Q} c_{1}^{Q}(\varphi)=-\partial c_{2}^{M}(\varphi)
$$


The first of these relations implies that the homology class $\left[c_{0}^{T}(\varphi)\right] \in H_{0}(T)$ belongs to the kernel ker $\Delta^{T}$ of the induced map $\Delta^{T}: H_{0}(T) \rightarrow H_{0}(Q)$. Consider the connecting homomorphism

$$
B: \operatorname{ker} \Delta^{T} \longrightarrow H_{1}(M) / \Delta^{Q}\left(H_{1}(Q)\right)
$$

defined by associating to the homology class of the 0-cycle $c_{0}^{T}$ such that $\Delta^{T} c_{0}^{T}=\partial c_{1}^{Q}$ the coset $\left[\Delta^{Q} c_{1}^{Q}\right]+\Delta^{Q}\left(H_{1}(Q)\right)$. The second of relations A.5) implies that $B\left(\left[c_{0}^{T}(\varphi)\right]\right)=0$. One may see again that the homology classes $\left[c_{0}^{T}(\varphi)\right]$ generate ker $B \subset H_{0}(T)$. Assuming relations (2.27) and (2.28), the chain of equations (A.2) now implies that

$$
\int_{\Sigma} \varphi^{*}\left(\iota_{a} \mathrm{H}\right)+\int_{\Gamma} \varphi^{*}\left(\iota_{a} \omega\right)=-\int_{\partial c_{1}^{Q}(\varphi)} k_{a}=-\int_{\Delta^{T} c_{0}^{T}(\varphi)} k_{a}=-\int_{c_{0}^{T}(\varphi)} \Delta_{T} k_{a} .
$$

Note that functions $k_{a}$ are defined modulo addition of $k_{a}^{\prime}$ such that $d k_{a}^{\prime}=\Delta_{Q} \kappa_{a}^{\prime}$ for some closed 1-forms $\kappa_{a}^{\prime}$. We have to show that the vanishing of the right-hand side of Eq. (A.6) for all $c_{1}^{Q}(\varphi)$ implies that functions $\Delta_{T} k_{a}$ on $T$ may be annihilated by such a modification. Not much seems to be needed since functions $\Delta_{T} k_{a}$ are locally constant,

$$
\mathrm{d} \Delta_{T_{n}} k_{a}=-\Delta_{T_{n}}\left(\iota_{a} \omega+\Delta_{Q} \kappa_{a}\right)=-\iota_{a} \Delta_{T_{n}} \omega=0
$$

due to Eq. 2.4). There is a cohomology exact sequence

$$
\operatorname{Hom}_{\mathbb{Z}}\left(H_{1}(M) / \Delta^{Q}\left(H_{1}(Q)\right), \mathbb{R}\right) \stackrel{B^{*}}{\longrightarrow} \operatorname{Hom}_{\mathbb{Z}}\left(\operatorname{ker} \Delta^{T}, \mathbb{R}\right) \longrightarrow \operatorname{Hom}_{\mathbb{Z}}(\operatorname{ker} B, \mathbb{R})
$$

obtained by applying the exact functor $\operatorname{Hom}_{\mathbb{Z}}(\cdot, \mathbb{R})$ to the homology exact sequence

$$
\operatorname{ker} B \hookrightarrow \operatorname{ker} \Delta^{T} \stackrel{B}{\longrightarrow} H_{1}(M) / \Delta^{Q}\left(H_{1}(Q)\right) .
$$

For 0-cycles $c_{0}^{T}$ such that $\Delta^{T} c_{0}^{T}=\partial c_{1}^{Q}$ for some 1-chain $c_{1}^{Q}$, the pairing

$$
\operatorname{ker} \Delta^{T} \ni\left[c_{0}^{T}\right] \mapsto \int_{c_{0}^{T}} \Delta_{T} k_{a} \in \mathbb{R}
$$

defines a $\mathbb{Z}$-homomorphism on ker $\Delta^{T}$ that, according to our assumption, vanishes when restricted to $\operatorname{ker} B$. It must then lie in the image of $\operatorname{Hom}_{\mathbb{Z}}\left(H_{1}(M) / \Delta^{Q}\left(H_{1}(Q)\right), \mathbb{R}\right)$ under the linear map $B^{*}$ dual to $B$. But $\operatorname{Hom}_{\mathbb{Z}}\left(H_{1}(M) / \Delta^{Q}\left(H_{1}(Q)\right), \mathbb{R}\right) \cong \operatorname{ker} \Delta_{Q} \subset H^{1}(M, \mathbb{R})$ and elements of ker $\Delta_{Q}$ are the cohomology classes of closed 1-forms $\kappa^{\prime}$ on $M$ such that there exists a function $k^{\prime}$ on $Q$ for which $\Delta_{Q} \kappa^{\prime}=d k^{\prime}$. One has

$$
\left\langle\left[c_{0}^{T}\right], B^{*}\left[\kappa^{\prime}\right]\right\rangle=\left\langle B\left[c_{0}^{T}\right],\left[\kappa^{\prime}\right]\right\rangle=\left\langle\left[\Delta^{Q} c_{1}^{Q}\right],\left[\kappa^{\prime}\right]\right\rangle=\int_{c_{1}^{Q}} \Delta_{Q} \kappa^{\prime}=\int_{\partial c_{1}^{Q}} k^{\prime}=\int_{c_{0}^{T}} \Delta_{T} k^{\prime} .
$$

It follows that through allowed modifications of functions $k_{a}$, adding to them appropriate functions $k_{a}^{\prime}$ with $d k_{a}^{\prime}=\Delta_{Q} \kappa_{a}^{\prime}$ for some closed 1-forms $\kappa_{a}^{\prime}$, one may achieve that the pairing of Eq. (A.10) vanishes. But then the cohomology exact sequence

$$
H^{0}(Q, \mathbb{R}) \stackrel{\Delta_{T}}{\longrightarrow} H^{0}(T, \mathbb{R}) \longrightarrow \operatorname{Hom}_{\mathbb{Z}}\left(\operatorname{ker} \Delta^{T}, \mathbb{R}\right)
$$

shows that by an eventual further modifications of functions $k_{a}$ that adds to them locally constant functions $k_{a}^{\prime}$, we may obtain relation (2.29).

Appendix B. Proofs of the "only if" parts of Proposition 4.9 And Theorem 4.12

We shall argue similarly as in Appendix A. Consider a closed world-sheet $\Sigma$ with a defect quiver $\Gamma$ without defect junctions and a triangulation of $\Sigma$ compatible with $\Gamma$. A gauge transformation $\chi: \Sigma \rightarrow \mathrm{G}$ and a network-field configuration $(\varphi \mid \Gamma)$, both restricted to $\Gamma$, determine a singular 1-cycle $c_{1}^{Q^{(1)}}(\chi, \varphi)$ in $Q^{(1)}=\mathrm{G} \times Q$. When both are restricted to $\Sigma \backslash \Gamma$ (and extended by the boundary values), they also define a singular 2-chain $c_{2}^{M^{(1)}}(\chi, \varphi)$ in $M^{(1)}=\mathrm{G} \times M$. Denoting by $\Delta^{Q^{(1)}}$ the combination $\left(\iota_{2}^{(1)}\right)_{*}-\left(\iota_{1}^{(1)}\right)_{*}$ of maps on chains originating from the $\iota_{\alpha}^{(1)}=\operatorname{id}_{\mathrm{G}} \times \iota_{\alpha}$, we have

$$
\Delta^{Q^{(1)}} c_{1}^{Q^{(1)}}(\chi, \varphi)=-\partial c_{2}^{M^{(1)}}(\chi, \varphi)
$$


cf. Eq. A.1 . Hence, the homology class $\left[c_{1}^{Q^{(1)}}(\chi, \varphi)\right]$ lies in the kernel ker $\Delta^{Q^{(1)}}$ of the induced map $\Delta^{Q^{(1)}}: H_{1}\left(Q^{(1)}\right) \rightarrow H_{1}\left(M^{(1)}\right)$. Besides, $\operatorname{ker} \Delta^{Q^{(1)}} \subset H_{1}\left(Q^{(1)}\right)$ may be generated by homology classes obtained that way. The triviality of the holonomy $\operatorname{Hol}_{D}\left(\left.(\chi, \varphi)\right|_{\Gamma}\right)$ then implies that the isomorphism class $[D] \in H^{1}\left(Q^{(1)}, \mathrm{U}(1)\right)$ of the flat line bundle $D$ is in the kernel of the right morphism of the the cohomology exact sequence

$$
H^{1}\left(M^{(1)}, \mathrm{U}(1)\right) \stackrel{\Delta_{Q}^{(1)}}{\longrightarrow} H^{1}\left(Q^{(1)}, \mathrm{U}(1)\right) \stackrel{r}{\longrightarrow} \operatorname{Hom}_{\mathbb{Z}}\left(\operatorname{ker} \Delta^{Q^{(1)}}, \mathrm{U}(1)\right),
$$

obtained by applying the exact functor $\operatorname{Hom}_{\mathbb{Z}}(\cdot, \mathrm{U}(1))$ to the exact sequence of $\mathbb{Z}$-modules

$$
\operatorname{ker} \Delta^{Q^{(1)}} \hookrightarrow H_{1}\left(Q^{(1)}\right) \stackrel{\Delta^{Q^{(1)}}}{\longrightarrow} H_{1}\left(M^{(1)}\right) .
$$

Thus, $[D]$ lies in the image of the left morphism of the exact sequence of Eq. (B.1). This means that $[D]$ is trivializable by a redefinition of the 1-isomorphism $\Upsilon$ of Eq. (4.7) by a flat bundle, cf. Proposition 2.8, completing the proof of the "only if" part of Proposition 4.9. Note that such a redefinition of $\Upsilon$ is fixed only modulo a flat bundle $D^{\prime}$ over $M^{(1)}$ with isomorphism class $\left[D^{\prime}\right]$ in the kernel of the left homomorphism in the exact sequence of Eq. (B.1), i.e. such that there exists a trivialisation $d^{\prime}$ of flat bundles $\Delta_{Q}^{(1)} D^{\prime}$ over $Q^{(1)}$. Since the choice of a 2-isomorphism $\Xi$ of Eq. (4.14) is given by a choice of a trivialisation of the bundle $D$, it is defined modulo trivializations $d^{\prime}$. Pulling back $d^{\prime}$ to $T^{(1)}$ with the help of $\Delta_{T}^{(1)}$, one obtains a trivialisation of the flat bundle $\Delta_{T}^{(1)} \Delta_{Q}^{(1)} D^{\prime}$ which is naturally trivial since each fibre of $D^{\prime}$ that appears in it is accompanied by the dual fibre. The two trivializations differ on $T_{n}^{(1)}$ by multiplication by a locally constant $\mathrm{U}(1)$-valued function $d_{n}^{\prime}$ that describes possible multiplicative ambiguities of the function $d_{n}$ defined by Eq. (4.16). We shall use such ambiguities below.

In order to prove the "only if" part of Theorem 4.12, we shall employ Proposition 4.10, proceeding similarly as in Appendix A. For a closed surface with an arbitrary defect quiver $\Gamma$ and a triangulation compatible with $\Gamma$, a gauge transformation $\chi: \Sigma \rightarrow G$ and a network-field configuration $(\varphi \mid \Gamma)$ determine a 0 -cycle $c_{0}^{T^{(1)}}(\chi, \varphi)$ in $T^{(1)}=\mathrm{G} \times T$, a 1 -chain $c_{1}^{Q^{(1)}}(\chi, \varphi)$ in $Q^{(1)}$ and a 2-chain $c_{2}^{M^{(1)}}(\chi, \varphi)$ in $M^{(1)}$ such that

$$
\Delta^{T^{(1)}} c_{0}^{T^{(1)}}(\chi, \varphi)=\partial c_{1}^{Q^{(1)}}(\chi, \varphi), \quad \Delta^{Q^{(1)}} c_{1}^{Q^{(1)}}(\chi, \varphi)=-\partial c_{2}^{M^{(1)}}(\chi, \varphi),
$$

with $\Delta^{T^{(1)}}$ defined similarly as $\Delta^{T}$, cf. Eq. A.5). This means that the homology class $\left[c_{0}^{T^{(1)}}(\chi, \varphi)\right]$ is in the kernel $\operatorname{ker} B^{(1)}$ of the homomorphism

$$
B^{(1)}: \operatorname{ker} \Delta^{T^{(1)}} \longrightarrow H_{1}\left(M^{(1)}\right) / \Delta^{Q^{(1)}}\left(H_{1}\left(Q^{(1)}\right)\right)
$$

defined as before, i.e. assigning to a homology class of a 0 -cycle $c_{0}^{T^{(1)}}$ such that $\Delta^{T^{(1)}} c_{0}^{T^{(1)}}=$ $\partial c_{1}^{Q^{(1)}}$ the coset $\left[\Delta^{Q^{(1)}} c_{1}^{Q^{(1)}}\right]+\Delta^{Q^{(1)}}\left(H_{1}\left(Q^{(1)}\right)\right)$. One may see that $\operatorname{ker} B^{(1)}$ is generated by classes $\left[c_{0}^{T^{(1)}}(\chi, \varphi)\right]$ (this follows from the fact that $\operatorname{ker} B$ was generated by classes $\left[c_{0}^{T}(\varphi)\right]$ ). The collection $\left(d_{n}\right)$ of locally constant $\mathrm{U}(1)$-valued functions may be viewed as an element of $H^{0}\left(T^{(1)}, \mathrm{U}(1)\right) \cong \operatorname{Hom}_{\mathbb{Z}}\left(H_{0}\left(T^{(1)}\right), \mathrm{U}(1)\right)$, and one has the identity

$$
\prod_{\jmath \in \mathfrak{V}_{\Gamma}}(\chi, \varphi)^{*} d_{n_{\jmath}}(\jmath)^{ \pm 1}=\left\langle\left[c_{0}^{T^{(1)}}(\chi, \varphi)\right],\left(d_{n}\right)\right\rangle,
$$

where the factor on the right-hand side of Eq. (4.20) describes an eventual violation of the gauge invariance. The triviality of that factor for all classes $\left[c_{0}^{T^{(1)}}(\chi, \varphi)\right]$ implies that $\left(d_{n}\right)$ defines an element in the kernel of the restriction homomorphism on the right of the cohomology exact sequence

$$
\begin{aligned}
\operatorname{Hom}_{\mathbb{Z}}\left(H_{1}\left(M^{(1)}\right) / \Delta^{Q^{(1)}}\left(H_{1}\left(Q^{(1)}\right)\right), \mathrm{U}(1)\right) \stackrel{\left(B^{(1)}\right)^{*}}{\longrightarrow} & \operatorname{Hom}_{\mathbb{Z}}\left(\operatorname{ker} \Delta^{T^{(1)}}, \mathrm{U}(1)\right) \\
& \longrightarrow \operatorname{Hom}_{\mathbb{Z}}\left(\operatorname{ker} B^{(1)}, \mathrm{U}(1)\right),
\end{aligned}
$$

and hence an element in the image of the left homomorphism. The group on the left of Eq. (B.2) is naturally identified with the kernel of the homomorphism $\Delta_{Q}^{(1)}: H^{1}\left(M^{(1)}, \mathrm{U}(1)\right) \rightarrow$ 
$H^{1}\left(Q^{(1)}, \mathrm{U}(1)\right)$. For $\left[D^{\prime}\right] \in H^{1}\left(M^{(1)}, \mathrm{U}(1)\right)$ in that kernel and for a 1-cycle $c_{0}^{T^{(1)}}$ such that $\Delta^{T^{(1)}} c_{0}^{T^{(1)}}=\partial c_{1}^{Q^{(1)}}$,

$$
\left\langle c_{0}^{T^{(1)}},\left(B^{(1)}\right)^{*}\left[D^{\prime}\right]\right\rangle=\left\langle B^{(1)}\left[c_{0}^{T^{(1)}}\right],\left[D^{\prime}\right]\right\rangle=\left\langle\Delta^{Q^{(1)}} c_{1}^{Q^{(1)}}, D^{\prime}\right\rangle=\left\langle\partial c_{1}^{Q^{(1)}}, d^{\prime}\right\rangle,
$$

where the expression on the right-hand side is identified with an element of $\mathrm{U}(1)$ using the fact that $\Delta^{Q^{(1)}} \partial c_{1}^{Q^{(1)}}=0$. But then

$$
\left\langle\partial c_{1}^{Q^{(1)}}, d^{\prime}\right\rangle=\left\langle c_{0}^{T^{(1)}}, \Delta_{T}^{(1)} d^{\prime}\right\rangle=\left\langle c_{0}^{T^{(1)}},\left(d_{n}^{\prime}\right)\right\rangle,
$$

where the right-hand side provides an identification of the middle term with an element of $\mathrm{U}(1)$. It then follows that by an allowed modification of functions $\left(d_{n}\right)$, we may achieve that

$$
\left\langle\left[c_{0}^{T^{(1)}}\right],\left(d_{n}\right)\right\rangle=1
$$

for all classes $\left[c_{0}^{T^{(1)}}\right] \in \operatorname{ker} \Delta^{T^{(1)}}$. The exact cohomology sequence

$$
H^{0}\left(Q^{(1)}, \mathrm{U}(1)\right) \stackrel{\Delta_{T}^{(1)}}{\longrightarrow} H^{0}\left(T^{(1)}, \mathrm{U}(1)\right) \longrightarrow \operatorname{Hom}_{\mathbb{Z}}\left(\operatorname{ker} \Delta^{T^{(1)}}, \mathrm{U}(1)\right)
$$

implies, in turn, that a further modification of the 2-isomorphism $\Xi$ by locally constant $\mathrm{U}(1)$ valued functions on $Q^{(1)}$ allows to get rid of functions $\left(d_{n}\right)$ altogether. This completes the proof of the "only if" part of Theorem 4.12 .

\section{Appendix C. Groupoids And Algebroids}

In this appendix, we recall several basic concepts from the theory of (Lie) groupoids and algebroids, of relevance to the discussion presented in the main text. The interested reader is urged to consult the literature on the subject, e.g., Refs. Mac87, MM03.

We begin with

Definition C.1. A groupoid is the septuple $\mathrm{Gr}=(\mathrm{Ob} \mathrm{Gr}, \mathrm{Mor} \mathrm{Gr}, s, t, \mathrm{Id}, \mathrm{Inv}, \circ)$ composed of a pair of sets: the object set $\mathrm{ObGr}$ and the arrow set Mor $\mathrm{Gr}$, and a quintuple of structure maps: the source map $s:$ Mor Gr $\rightarrow \mathrm{ObGr}$ and the target map $t:$ Mor Gr $\rightarrow$ ObGr, the unit map Id : ObGr $\rightarrow$ Mor Gr $: m \mapsto \mathrm{Id}_{m}$, the inverse map Inv : Mor Gr $\rightarrow$

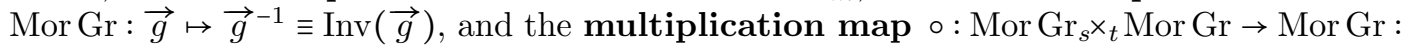
$(\vec{g}, \vec{h}) \mapsto \vec{g} \circ \vec{h}$. Here, Mor $\operatorname{Gr}_{s} \times_{t}$ Mor Gr is the subset of composable morphisms,

$$
\text { Mor } \operatorname{Gr}_{s} \times_{t} \operatorname{Mor} \mathrm{Gr}=\{(\vec{g}, \vec{h}) \in \operatorname{Mor} \mathrm{Gr} \times \operatorname{Mor} \mathrm{Gr} \quad \mid s(\vec{g})=t(\vec{h})\} .
$$

The structure maps satisfy the consistency conditions (whenever the expressions are welldefined):

(i) $s(\vec{g} \circ \vec{h})=s(\vec{h}), t(\vec{g} \circ \vec{h})=t(\vec{g})$;

(ii) $(\vec{g} \circ \vec{h}) \circ \vec{k}=\vec{g} \circ(\vec{h} \circ \vec{k})$;

(iii) $\operatorname{Id}_{t(\vec{g})} \circ \vec{g}=\vec{g}=\vec{g} \circ \operatorname{Id}_{s(\vec{g})}$;

(iv) $s\left(\vec{g}^{-1}\right)=t(\vec{g}), t\left(\vec{g}^{-1}\right)=s(\vec{g}), \vec{g} \circ \vec{g}^{-1}=\operatorname{Id}_{t(\vec{g})}, \vec{g}^{-1} \circ \vec{g}=\operatorname{Id}_{s(\vec{g})}$.

Thus, a groupoid is a (small) category with all morphisms invertible.

A morphism between two groupoids $\mathrm{Gr}_{i}, i=1,2$ is a functor $\Phi: \mathrm{Gr}_{1} \rightarrow \mathrm{Gr}_{2}$.

A Lie groupoid is a groupoid whose object and arrow sets are smooth manifolds, whose structure maps are smooth, and whose source and target maps are surjective submersions. A morphism between two Lie groupoids is a functor between them with smooth object and morphism components.

We also have

Definition C.2. Let $\mathscr{M}$ be a smooth manifold. A Lie algebroid over the base $\mathscr{M}$ is a triple $\mathfrak{G r}=\left(V,[\cdot, \cdot], \alpha_{\mathrm{T} \mathscr{M}}\right)$ composed of a vector bundle $\pi_{V}: V \rightarrow \mathscr{M}$, a Lie bracket $[\cdot, \cdot \cdot]$ on the vector space $\Gamma(V)$ of its sections, and a bundle map $\alpha_{\mathrm{T} \mathscr{M}}: V \rightarrow \mathrm{T} \mathscr{M}$, termed the anchor (map). These are required to have the following properties:

(i) the induced map $\Gamma\left(\alpha_{\mathrm{T} \mathscr{M}}\right): \Gamma(V) \rightarrow \Gamma(\mathrm{T} \mathscr{M})$, to be denoted by the same symbol $\alpha_{\mathrm{T} \mathscr{M}}$ below, is a Lie-algebra homomorphism; 
(ii) $[\cdot, \cdot]$ obeys the Leibniz identity

$$
[X, f Y]=f[X, Y]+\Gamma\left(\alpha_{\boldsymbol{\top} \mathscr{M}}\right)(X)(f) Y
$$

for all $X, Y \in \Gamma(V)$ and any $f \in C^{\infty}(\mathscr{M}, \mathbb{R})$.

A morphism between two Lie algebroids $\mathfrak{G r}_{i}=\left(V_{i},[\cdot, \cdot]_{i}, \alpha_{\mathrm{T} \mathscr{M} i}\right), i=1,2$ (over the same base $\mathscr{M})$ is a bundle map $\phi: V_{1} \rightarrow V_{2}$ that satisfies the relations

$$
\alpha_{\mathrm{T} \mathscr{M} 1}=\alpha_{\mathrm{T} \mathscr{M} 2} \circ \phi, \quad[\cdot, \cdot]_{1}=[\cdot, \cdot]_{2} \circ(\phi \times \phi) .
$$

Furthermore, we shall need

Definition C.3. Let $\mathrm{Gr}=(\mathrm{ObGr}, \mathrm{Mor} \mathrm{Gr}, s, t, \mathrm{Id}, \mathrm{Inv}, \circ)$ be a Lie groupoid. Denote by $\mathrm{d} s$ and $\mathrm{d} t$ the tangents of the source map and the target map, respectively, and by $\mathrm{d} R_{\vec{g}}, \vec{g} \epsilon$ Mor Gr the tangent of the smooth map

$$
R_{\vec{g}}: s^{-1}(\{t(\vec{g})\}) \rightarrow s^{-1}(\{s(\vec{g})\}): \vec{h} \mapsto R_{\vec{g}}(\vec{h}):=\vec{h} \circ \vec{g} .
$$

Furthermore, let $\mathfrak{X}_{\text {inv }}^{s}($ Mor Gr) denote the vector space of right Gr-invariant vector fields on Mor Gr, given by

$$
\mathfrak{X}_{\text {inv }}^{s}(\text { Mor Gr })=\{\mathscr{V} \in \Gamma(\operatorname{ker} \mathrm{d} s) \quad \mid \quad \mathrm{d} R \cdot(\mathscr{V})=\mathscr{V}\} .
$$

The tangent algebroid of $\mathrm{Gr}$ is the Lie algebroid $\mathfrak{g r}=\left(\operatorname{Id}^{*} \operatorname{ker} \mathrm{d} s,[\cdot, \cdot], \alpha_{\mathrm{T}(\mathrm{ObGr})}\right)$ over Ob Gr with the anchor $\alpha_{\mathrm{T}(\mathrm{ObGr})}$ inducing the map $\mathrm{d} t \circ i$ between spaces of sections, defined in terms of the canonical vector-space isomorphism

$$
i: \Gamma\left(\mathrm{Id}^{*} \operatorname{ker} \mathrm{d} s\right) \stackrel{\cong}{\longrightarrow} \mathfrak{X}_{\text {inv }}^{s}(\text { Mor Gr }),
$$

and with the Lie bracket given by the unique bracket on $\Gamma\left(\operatorname{Id}^{*} \operatorname{ker} \mathrm{d} s\right)$ for which $i$ is an isomorphism of Lie algebras.

\section{Appendix D. A proof of Theorem 6.4}

By way of proof, we present a detailed reconstruction of the tangent algebroid $\mathfrak{g} \times \mathscr{F}$ along the lines of the abstract definition C.3, specialising the general concepts to the case of interest, $\mathrm{Gr}=\mathrm{S}_{\mathfrak{B}} \equiv \mathrm{G} \ltimes \mathscr{F}$. We commence by extracting the vector sub-bundle

$$
\mathrm{T}^{s}(\mathrm{G} \times \mathscr{F}):=\operatorname{ker} \mathrm{d} s \subset \mathrm{T}(\mathrm{G} \times \mathscr{F})
$$

of the tangent bundle $\pi: \mathrm{T}(\mathrm{G} \times \mathscr{F}) \rightarrow \mathrm{G} \times \mathscr{F}$, defined in terms of the tangent map $\mathrm{d} s$ and hence spanned by vector fields tangent to the $s$-fibres in $\mathrm{G} \times \mathscr{F}$. In the case in hand, these vector fields are given by $C^{\infty}(\mathrm{G} \times \mathscr{F}, \mathbb{R})$-linear combinations $f^{i}(g, m) \mathscr{V}_{i}(g)$ of vector fields on G. On sections $\mathfrak{X}^{s}(\mathrm{G} \times \mathscr{F}):=\Gamma\left(\mathrm{T}^{s}(\mathrm{G} \times \mathscr{F})\right)$, we have the natural (right) fibre-wise

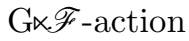

$$
\rho: \mathfrak{X}^{s}(\mathrm{G} \times \mathscr{F})_{\mu_{\pi}} \times_{t}(\mathrm{G} \times \mathscr{F}) \rightarrow \mathfrak{X}^{s}(\mathrm{G} \times \mathscr{F}):(\mathscr{V}, \vec{g}) \mapsto \mathrm{d} R_{\vec{g}}(\mathscr{V})=: \rho_{\vec{g}}(\mathscr{V}) \equiv \mathscr{V} \cdot \vec{g}
$$

with momentum

$$
\mu_{\pi}:=s \circ \pi,
$$

defined in terms of the tangent $\mathrm{d} R$ of the right action

$$
R:(\mathrm{G} \times \mathscr{F})_{\mu_{R}} \times(\mathrm{G} \times \mathscr{F}) \rightarrow \mathrm{G} \times \mathscr{F}:(\vec{g}, \vec{h}) \mapsto \vec{g} \circ \vec{h}=: R_{\vec{h}}(\vec{g})
$$

with momentum

$$
\mu_{R}:=s .
$$

Above, and in what follows, we employ

Convention D.1. Given a pair $f_{i}: \mathscr{M}_{i} \mapsto \mathscr{N}, i=1,2$ of maps from the respective domains $\mathscr{M}_{i}$ to the common codomain $\mathscr{N}$, the symbol $\mathscr{M}_{f_{1}} \times_{f_{2}} \mathscr{M}_{2}$ denotes the fibred product

$$
\mathscr{M}_{1 f_{1} \times f_{2}} \mathscr{M}_{2}=\left\{\left(m_{1}, m_{2}\right) \in \mathscr{M}_{1} \times \mathscr{M}_{2} \quad \mid \quad f_{1}\left(m_{1}\right)=f_{2}\left(m_{2}\right)\right\} .
$$


The restriction to the sub-bundle $\mathrm{T}^{s}(\mathrm{G} \times \mathscr{F})$ ensures that $\rho$ covers the right action $R$ on the base,

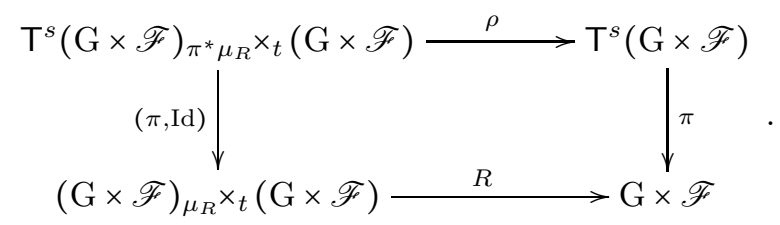

Among sections $\mathfrak{X}^{s}(\mathrm{G} \times \mathscr{F})$, we subsequently distinguish those, denoted jointly by $\mathfrak{X}_{\text {inv }}^{s}(\mathrm{G} \times$ $\mathscr{F}) \equiv \Gamma\left(\mathrm{T}_{\text {inv }}^{s}(\mathrm{G} \times \mathscr{F})\right)$, which are invariant under $\rho$, i.e. such that

$$
\mathrm{d} R_{\vec{h}}(\mathscr{V})(\vec{g} \circ \vec{h}) \equiv \mathscr{V}(\vec{g}) \cdot \vec{h}=\mathscr{V}(\vec{g} \circ \vec{h}) .
$$

They close to a Lie subalgebra of the Lie algebra $\mathfrak{X}(\mathrm{G} \times \mathscr{F})$ of vector fields on $\mathrm{G} \times \mathscr{F}$ since

$$
\mathrm{d} R_{\vec{h}}([\mathscr{V}, \mathscr{W}])=\left[\mathrm{d} R_{\vec{h}}(\mathscr{V}), \mathrm{d} R_{\vec{h}}(\mathscr{W})\right]=[\mathscr{V}, \mathscr{W}],
$$

and the subalgebra homomorphically projects, along $\mathrm{d} t$, to the Lie algebra $\mathfrak{X}(\mathscr{F})$ of vector fields on $\mathscr{F}$, a straightforward consequence of the previous property and of the identity

$$
\mathrm{d} t(\mathscr{V}(\vec{g}))=\mathrm{d} t\left(\mathrm{~d} R_{\vec{g}}\left(\mathscr{V}\left(\operatorname{Id}_{t(\vec{g})}\right)\right)\right)=\mathrm{d}\left(t \circ R_{\vec{g}}\right)\left(\mathscr{V}\left(\operatorname{Id}_{t(\vec{g})}\right)\right)=\mathrm{d} t\left(\mathscr{V}\left(\operatorname{Id}_{t(\vec{g})}\right)\right)
$$

The subalgebra may be reconstructed as follows: Represent an arbitrary right-invariant vector field as a $C^{\infty}(\mathrm{G} \times \mathscr{F}, \mathbb{R})$-linear combination of the basic right-invariant vector fields $R_{a}, a=$ $1,2, \ldots, \operatorname{dim} \mathfrak{g}$ on $\mathrm{G}$ as discussed above. We readily establish the defining property of the functional coefficients in this decomposition of a right-invariant vector field:

$$
f^{i}\left(g \cdot h, h^{-1} \cdot m\right)=f^{i}(g, m) \quad \Longrightarrow \quad f^{i}(g, m)=f^{i}(e, g . m),
$$

which shows that each $f^{i}$ factors through a function $\bar{f}^{i}=f^{i} \circ \operatorname{Id} \in C^{\infty}(\mathscr{F}, \mathbb{R})$ as

$$
f^{i}=\bar{f}^{i} \circ t \text {. }
$$

Thus, altogether, a right-invariant vector field on $\mathrm{G} \times \mathscr{F}$ can be written in the form

$$
\mathscr{V}=\left(v^{a} \circ t\right)\left(R_{a} \circ \mathrm{pr}_{1}\right), \quad v^{a} \in C^{\infty}(\mathscr{F}, \mathbb{R}), \quad \operatorname{pr}_{1}: \mathrm{G} \times \mathscr{F} \rightarrow \mathrm{G} .
$$

This explicitly demonstrates the isomorphism of vector spaces

$$
\mathfrak{X}_{\text {inv }}^{s}(\mathrm{G} \times \mathscr{F}) \cong \Gamma\left(\mathrm{Id}^{*} \mathrm{~T}^{s}(\mathrm{G} \times \mathscr{F})\right) .
$$

Indeed, in general, every right-invariant vector field on $\mathrm{G} \times \mathscr{F}$ defines a vector field on $\operatorname{Id}(\mathscr{F})$ via restriction, and, conversely, every vector field $\mathscr{V} \in \Gamma\left(\operatorname{Id}^{*} \mathrm{~T}^{s}(\mathrm{G} \times \mathscr{F})\right)$ extends to a unique right-invariant vector field $\overrightarrow{\mathscr{V}}$ on the arrow manifold as

$$
\overrightarrow{\mathscr{V}}(\vec{g}):=\mathscr{V}\left(\operatorname{Id}_{t(\vec{g})}\right) \cdot \vec{g}
$$

The bijectivity of this correspondence follows from the fact that a right-invariant vector field on $\mathrm{G} \times \mathscr{F}$ is completely determined by its restriction to $\operatorname{Id}(\mathscr{F})$. Note also that the correspondence is compatible with the $C^{\infty}(\mathscr{F}, \mathbb{R})$-module structure on both vector spaces as

$$
\overrightarrow{f \mathscr{V}}=(f \circ t) \overrightarrow{\mathscr{V}}, \quad f \in C^{\infty}(\mathscr{F}, \mathbb{R})
$$

cf. Eq. (D.3). The vector bundle

$$
\mathrm{Id}^{*} \mathrm{~T}_{\text {inv }}^{s}(\mathrm{G} \times \mathscr{F}) \rightarrow \mathscr{F}
$$

is the first ingredient of the Lie algebroid under reconstruction. By virtue of Eq. (D.3), we obtain, in the case of interest,

$$
\mathrm{Id}^{*} \mathrm{~T}_{\text {inv }}^{s}(\mathrm{G} \times \mathscr{F}) \cong \mathfrak{g} \times \mathscr{F} .
$$

The next step consists in defining the anchor map

$$
\alpha_{\mathrm{T} \mathscr{F}}: \mathrm{Id}^{*} \mathrm{~T}_{\text {inv }}^{s}(\mathrm{G} \times \mathscr{F}) \rightarrow \mathrm{T} \mathscr{F}
$$

that induces a Lie-algebra homomorphism between the Lie-bracket algebras of sections. Comparison with Eqs. (D.1)-(D.2) immediately suggests the choice

$$
\alpha_{\mathrm{T} \mathscr{F}}(\mathscr{V})=\mathrm{d} t(\overrightarrow{\mathscr{V}}) .
$$


We find, for the restriction $\mathscr{R}_{a}$ of the right-invariant vector field $R_{a} \circ \mathrm{pr}_{1}$,

$$
\begin{aligned}
\left(\alpha_{\mathrm{\top} \mathscr{F}}\left(\mathscr{R}_{a}\right)(f)\right)(m) & \equiv t^{*}\left(\alpha_{\mathrm{\top} \mathscr{F}}\left(\mathscr{R}_{a}\right)(f)\right)(e, m)=t^{*}\left(\imath_{t_{*} R_{a}} \mathrm{~d} f\right)(e, m)=\left(\imath_{R_{a}} \mathrm{~d}\left(t^{*} f\right)\right)(e, m) \\
& =\left.\left.\frac{\mathrm{d}}{\mathrm{d} s}\right|_{s=0}\left(t^{*} f\right)\left(\mathrm{e}^{-s t_{a}} \cdot e, m\right) \equiv \frac{\mathrm{d}}{\mathrm{d} s}\right|_{s=0} f\left(\mathrm{e}^{-s t_{a}} \cdot m\right)=\left(\mathscr{F}^{\mathscr{K}} \mathscr{K}_{a}(f)\right)(m),
\end{aligned}
$$

or simply

$$
\mathrm{d} t\left(R_{a} \circ \operatorname{pr}_{1}\right)=\alpha_{\mathrm{T} \mathscr{F}}\left(\mathscr{R}_{a}\right)={ }^{\mathscr{F}} \mathscr{K}_{a},
$$

with $\mathscr{F} \mathscr{K}_{a}, a=1,2, \ldots, \operatorname{dim} \mathfrak{g}$ generating the infinitesimal action of $\mathrm{G}$ on $C^{\infty}(\mathscr{F}, \mathbb{R})$, as in Definition 2.10. This fixes the anchor map completely.

At this stage, it remains to identify the unique Lie bracket on $\Gamma(\mathfrak{g} \times \mathscr{F}) \equiv C^{\infty}(M, \mathfrak{g})$ with the Leibniz property for which $\mathfrak{X}_{\text {inv }}^{s}(\mathrm{G} \times \mathscr{F}) \cong \Gamma(\mathfrak{g} \times \mathscr{F})$ is a Lie-algebra isomorphism, that is the unique bracket such that

$$
\begin{aligned}
& \overrightarrow{[\mathscr{V}, f \mathscr{W}}]_{\mathfrak{g} \times \mathscr{F}}=[\overrightarrow{\mathscr{V}}, \overrightarrow{f \mathscr{W}}]=[\overrightarrow{\mathscr{V}},(f \circ t) \overrightarrow{\mathscr{W}}]=(f \circ t)[\overrightarrow{\mathscr{V}}, \overrightarrow{\mathscr{W}}]+\overrightarrow{\mathscr{V}}(f \circ t) \overrightarrow{\mathscr{W}} \\
& =(f \circ t) \overrightarrow{[\mathscr{V}, \mathscr{W}}_{\mathfrak{g} \times \mathscr{F}}+(\mathrm{d} t(\overrightarrow{\mathscr{V}})(f) \circ t) \overrightarrow{\mathscr{W}}=\overrightarrow{f[\mathscr{V}, \mathscr{W}]_{\mathfrak{g} \times \mathscr{F}}+\mathrm{d} t(\overrightarrow{\mathscr{V}})(f) \mathscr{W}},
\end{aligned}
$$

whence

$$
[\mathscr{V}, f \mathscr{W}]_{\mathfrak{g} \ltimes \mathscr{F}}=f[\mathscr{V}, \mathscr{W}]_{\mathfrak{g} \ltimes \mathscr{F}}+\alpha_{\mathrm{\top} \mathscr{F}}(\mathscr{V})(f) \mathscr{W} .
$$

Using the last result, in conjunction with the obvious implication

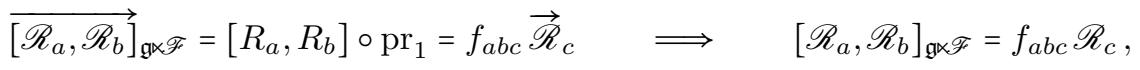

we ultimately derive the general expression

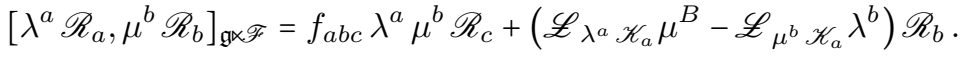

Thus, after the dust has cleared, we find the full-fledged structure of the tangent algebroid

$$
\mathfrak{g} \times \mathscr{F}=\left(\oplus_{a=1}^{\operatorname{dim} \mathfrak{g}} C^{\infty}(\mathscr{F}, \mathbb{R}) \mathscr{R}_{a},[\cdot, \cdot]_{\mathfrak{g} \ltimes \mathscr{F}}, \alpha_{\mathrm{T} \mathscr{F}}\right) .
$$

of the action groupoid $\mathrm{G} \ltimes \mathscr{F}$, also termed the action algebroid. It is now a matter of a straightforward calculation to check that the map

$$
\iota: \mathfrak{S}_{\mathfrak{B}} \rightarrow \mathfrak{g} \ltimes \mathscr{F}: \lambda^{a} \mathfrak{K}_{a} \mapsto \lambda^{a} \mathscr{R}_{a}
$$

is an isomorphism of Lie algebroids over the target space.

\section{Appendix E. A proof of Proposition 7.2}

We first compute

$$
D_{3} \mathscr{L}_{a} b=\mathscr{L}_{a}\left(\left.\mathrm{H}\right|_{\mathcal{O}_{i}^{M}}, 0,0,0,0\right)=0,
$$

whereby it becomes clear that condition (2.27) lifts the obstruction to trivialising the 3-cocycle $\mathscr{L}_{a} b$ in the Deligne hypercohomology. Indeed, we have

$$
\begin{aligned}
\mathscr{L}_{a} B_{i} & =\left.\imath_{a} \mathrm{H}\right|_{\mathcal{O}_{i}^{M}}+\mathrm{d}\left(\imath_{a} B_{i}\right)=\mathrm{d}\left(-\left.\kappa_{a}\right|_{\mathcal{O}_{i}^{M}}+\imath_{a} B_{i}\right), \\
\mathscr{L}_{a} A_{i j} & =\left.\imath_{a}\left(B_{j}-B_{i}\right)\right|_{\mathcal{O}_{i j}^{M}}+\mathrm{d}\left(\imath_{a} A_{i j}\right), \\
\mathscr{L}_{a} h_{i j k} & =\left.\imath_{a}\left(-A_{j k}+A_{i k}-A_{i j}\right)\right|_{\mathcal{O}_{i j k}^{M}}, \\
\mathscr{L}_{a} s_{i j k l} & =0,
\end{aligned}
$$

and so

$$
\mathscr{L}_{a} b=D_{2} \Upsilon_{a} .
$$

The last identity, in conjunction with Eq. (7.7), implies, in turn,

$$
D_{2}\left(\mathscr{L}_{a} \Upsilon_{b}-\mathscr{L}_{b} \Upsilon_{a}\right)=\left[\mathscr{L}_{a}, \mathscr{L}_{b}\right] b=f_{a b c} \mathscr{L}_{c} b=D_{2}\left(f_{a b c} \Upsilon_{c}\right) \text {, }
$$

or, simply,

$$
\mathscr{L}_{a} \Upsilon_{b}-\mathscr{L}_{b} \Upsilon_{a}-f_{a b c} \Upsilon_{c} \in \operatorname{ker} D_{2}
$$


This time, it is condition (3.6) that turns the latter 2-cocycle into a 2-coboundary. We compute

$$
\mathscr{L}_{a}\left(-\left.\kappa_{b}\right|_{\mathcal{O}_{i}^{M}}+\imath_{b} B_{i}\right)=f_{a b c}\left(-\left.\kappa_{c}\right|_{\mathcal{O}_{i}^{M}}+\imath_{c} B_{i}\right)+\imath_{b} \mathscr{L}_{a} B_{i}
$$

and

$$
\begin{aligned}
\mathscr{L}_{b}\left(-\left.\kappa_{a}\right|_{\mathcal{O}_{i}^{M}}+\imath_{a} B_{i}\right) & =\left.f_{a b c} \kappa_{c}\right|_{\mathcal{O}_{i}^{M}}+\mathrm{d}\left(\imath_{b} \imath_{a} B_{i}\right)+\imath_{b} \mathrm{~d}\left(\imath_{a} B_{i}\right) \\
& =\left.f_{a b c} \kappa_{c}\right|_{\mathcal{O}_{i}^{M}}+\mathrm{d}\left(\imath_{b} \imath_{a} B_{i}\right)+\imath_{b} \mathscr{L}_{a} B_{i}+\left.\imath_{b} \mathrm{~d} \kappa_{a}\right|_{\mathcal{O}_{i}^{M}} \\
& =\imath_{b} \mathscr{L}_{a} B_{i}+\mathrm{d}\left(-\left.\left(\imath_{b} \kappa_{a}\right)\right|_{\mathcal{O}_{i}^{M}}+\imath_{b} \imath_{a} B_{i}\right),
\end{aligned}
$$

whence

$$
\begin{aligned}
& \mathscr{L}_{a}\left(-\left.\kappa_{b}\right|_{\mathcal{O}_{i}^{M}}+\imath_{b} B_{i}\right)-\mathscr{L}_{b}\left(-\left.\kappa_{a}\right|_{\mathcal{O}_{i}^{M}}+\imath_{a} B_{i}\right) \\
= & f_{a b c}\left(-\left.\kappa_{c}\right|_{\mathcal{O}_{i}^{M}}+\imath_{c} B_{i}\right)+\mathrm{d}\left(\left.\mathrm{c}_{b a}\right|_{\mathcal{O}_{i}^{M}}+\imath_{a} \imath_{b} B_{i}\right) .
\end{aligned}
$$

Similarly, we find

$$
\mathscr{L}_{a}\left(\imath_{b} A_{i j}\right)-\mathscr{L}_{b}\left(\imath_{a} A_{i j}\right)=f_{a b c} \imath_{c} A_{i j}+\left.\imath_{b} \imath_{a}\left(B_{j}-B_{i}\right)\right|_{\mathcal{O}_{i j}^{M}},
$$

so that, altogether,

$$
\mathscr{L}_{a} \Upsilon_{B}-\mathscr{L}_{b} \Upsilon_{a}=f_{a b c} \Upsilon_{c}+D_{1} \gamma_{a b}
$$

In the last step of this descent, we establish, with the help of the Jacobi identity for the structure constants $f_{a b c}$,

$$
\begin{aligned}
& D_{1}\left(\mathscr{L}_{a} \gamma_{b c}-\mathscr{L}_{b} \gamma_{a c}+\mathscr{L}_{c} \gamma_{a b}\right) \\
= & \mathscr{L}_{a}\left(\mathscr{L}_{b} \Upsilon_{c}-\mathscr{L}_{c} \Upsilon_{b}\right)-\mathscr{L}_{b}\left(\mathscr{L}_{a} \Upsilon_{c}-\mathscr{L}_{c} \Upsilon_{a}\right)+\mathscr{L}_{c}\left(\mathscr{L}_{a} \Upsilon_{b}-\mathscr{L}_{b} \Upsilon_{a}\right) \\
& -f_{b c d} \mathscr{L}_{a} \Upsilon_{d}+f_{a c d} \mathscr{L}_{b} \Upsilon_{d}-f_{a b d} \mathscr{L}_{c} \Upsilon_{d} \\
= & f_{a b d}\left(\mathscr{L}_{d} \Upsilon_{c}-\mathscr{L}_{c} \Upsilon_{d}\right)-f_{a c d}\left(\mathscr{L}_{d} \Upsilon_{b}-\mathscr{L}_{b} \Upsilon_{d}\right)+f_{b c d}\left(\mathscr{L}_{d} \Upsilon_{a}-\mathscr{L}_{a} \Upsilon_{d}\right) \\
= & D_{1}\left(f_{a b d} \gamma_{d c}-f_{a c d} \gamma_{d b}+f_{b c d} \gamma_{d a}\right),
\end{aligned}
$$

and so we may seek to trivialise the 1-cocycle

$$
\mathscr{L}_{a} \gamma_{b c}-\mathscr{L}_{b} \gamma_{a c}+\mathscr{L}_{c} \gamma_{a b}-f_{a b d} \gamma_{d c}+f_{a c d} \gamma_{d b}-f_{b c d} \gamma_{d a} \in \operatorname{ker} D_{1}
$$

A direct computation, using the shorthand notation $Y_{a, i}:=-\left.\kappa_{a}\right|_{\mathcal{O}_{i}^{M}}+\imath_{a} B_{i}$ alongside the relations

$$
g_{a b, i}=-\imath_{b} Y_{a, i}, \quad g_{a b, i}+g_{b a, i}=2 c_{(a b), i}
$$

satisfied by $g_{a b, i}:=\left.\mathrm{c}_{b a}\right|_{\mathcal{O}_{i}^{M}}+\imath_{a} \imath_{b} B_{i}$ with

$$
c_{(a b), i}=\left.\frac{1}{2}\left(c_{a b}+c_{b a}\right)\right|_{\mathcal{O}_{i}^{M}}, \quad \mathrm{~d} c_{(a b), i}=0,
$$

yields

$$
\begin{array}{r}
\mathscr{L}_{a} g_{b c, i}-\mathscr{L}_{b} g_{a c, i}+\mathscr{L}_{c} g_{a b, i} \\
=\imath_{a} \mathrm{~d} g_{b c, i}-\imath_{b} \mathrm{~d} g_{a c, i}+\imath_{c}\left(\mathscr{L}_{a} Y_{b, i}-\mathscr{L}_{b} Y_{a, i}-f_{a b d} Y_{d, i}\right) \\
=\imath_{a} \mathrm{~d} g_{b c, i}-\imath_{b} \mathrm{~d} g_{a c, i}+\imath_{c} \mathscr{L}_{a} Y_{b, i}-\imath_{b} \mathrm{~d}\left(\imath_{c} Y_{a, i}\right) \\
+f_{b c d} \imath_{d} Y_{a, i}-f_{a b d} \imath_{c} Y_{d, i} \\
=\imath_{a} \mathrm{~d} g_{b c, i}-\imath_{b}\left(\mathscr{L}_{a} Y_{c, i}-f_{a c d} Y_{d, i}\right)+\imath_{c} \mathscr{L}_{a} Y_{b, i}+\imath_{b} \imath_{c} \mathrm{~d} Y_{a, i} \\
+f_{b c d} \imath_{d} Y_{a, i}-f_{a b d} \imath_{c} Y_{d, i} \\
=\imath_{a} \mathrm{~d}\left(\imath_{b} Y_{c, i}\right)-\mathscr{L}_{a}\left(\imath_{b} Y_{c, i}\right)+\imath_{c} \mathscr{L}_{a} Y_{b, i}+\imath_{b} \imath_{c} \mathrm{~d} Y_{a, i}
\end{array}
$$




$$
\begin{array}{r}
+f_{a b d} \imath_{d} Y_{c, i}+f_{a c d} \imath_{b} Y_{d, i}+f_{b c d} \imath_{d} Y_{a, i}-f_{a b d} \imath_{c} Y_{d, i} \\
=f_{a b d} g_{d c, i}-f_{a c d} g_{d b, i}+f_{b c d} g_{d a, i}-2 f_{a b d} c_{(d c), i}-2 f_{b c d} c_{(d a), i} \\
+\imath_{c} \mathscr{L}_{a} Y_{b, i}+\imath_{b} \imath_{c} \mathrm{~d} Y_{a, i}-f_{a b d} \imath_{c} Y_{d, i} .
\end{array}
$$

Taking a closer look at the expression in the last line, we find

$$
\begin{array}{r}
\imath_{c} \mathscr{L}_{a} Y_{b, i}+\imath_{b} \imath_{c} \mathrm{~d} Y_{a, i}-f_{a b d} \imath_{c} Y_{d, i} \\
=-\left.f_{a b d} \mathrm{c}_{c d}\right|_{\mathcal{O}_{i}^{M}}+\imath_{c} \mathscr{L}_{a}\left(\imath_{b} B_{i}\right)+\left.\imath_{b} \imath_{c} \imath_{a} \mathrm{H}\right|_{\mathcal{O}_{i}^{M}} \\
+\imath_{b} \imath_{c} \mathrm{~d}\left(\imath_{a} B_{i}\right)-f_{a b d} \imath_{c}\left(-\left.\kappa_{d}\right|_{\mathcal{O}_{i}^{M}}+\imath_{d} B_{i}\right) \\
=\imath_{c} \imath_{b} \mathscr{L}_{a} B_{i}+\left.\imath_{b} \imath_{c} \imath_{a} \mathrm{H}\right|_{\mathcal{O}_{i}^{M}}+\imath_{b} \imath_{c} \mathrm{~d}\left(\imath_{a} B_{i}\right)=0 .
\end{array}
$$

Next, upon using the identity

$$
f_{b c d} c_{(d a), i}+f_{b a d} c_{(d c), i}=\mathscr{L}_{b} c_{(a c), i} \equiv \iota_{b} \mathrm{~d} c_{(a c), i}=0,
$$

we ultimately reduce Eq. (E.1) to the relation

$$
\begin{array}{r}
\mathscr{L}_{a} g_{b c, i}-\mathscr{L}_{b} g_{a c, i}+\mathscr{L}_{c} g_{a b, i} \\
=f_{a b d} g_{d c, i}-f_{a c d} g_{d b, i}+f_{b c d} g_{d a, i}-4 f_{a b d} c_{(d c), i},
\end{array}
$$

which explicitly identifies the (local) constants $c_{(a b), i}$ as the last obstruction to the $\mathfrak{g}$ equivariance of the gerbe $\mathcal{G}$ and thus concludes the proof.

\section{Appendix F. A proof of Proposition 7.3}

First of all, we have the identity

$$
D_{2} \mathscr{L}_{a} p=\check{\Delta}_{Q} \mathscr{L}_{a} b+\mathscr{L}_{a} \bar{\omega}=D_{2} \check{\Delta}_{Q} \Upsilon_{a},
$$

and the obstruction to trivialising the 2-cocycle

$$
\mathscr{L}_{a} p-\check{\Delta}_{Q} \Upsilon_{a} \in \operatorname{ker} D_{2}
$$

is lifted by imposing Eq. (2.28). Indeed, we have

$$
\begin{aligned}
\mathscr{L}_{a} P_{i} & =\mathrm{d}\left(\imath_{a} P_{i}\right)+\check{\Delta}_{Q}\left(\imath_{a} B_{i}\right)+\left.\imath_{a} \omega\right|_{\mathcal{O}_{i}^{Q}}=\mathrm{d}\left(-k_{a}+\imath_{a} P_{i}\right)+\check{\Delta}_{Q} Y_{a, i} \\
\mathscr{L}_{a} k_{i j} & =\imath_{a} \mathrm{~d} k_{i j}=\left.\imath_{a}\left(P_{i}-P_{j}\right)\right|_{\mathcal{O}_{i j}^{Q}}+\check{\Delta}_{Q}\left(\imath_{a} A_{i j}\right) \\
\mathscr{L}_{a} r_{i j k} & =0,
\end{aligned}
$$

and so we obtain

$$
\mathscr{L}_{a} p=\check{\Delta}_{Q} \Upsilon_{a}+D_{1} \Xi_{a}
$$

Similarly, given

$$
\begin{aligned}
D_{1}\left(\mathscr{L}_{a} \Xi_{b}-\mathscr{L}_{b} \Xi_{a}\right) & =\left[\mathscr{L}_{a}, \mathscr{L}_{b}\right] p-\check{\Delta}_{Q}\left(\mathscr{L}_{a} \Upsilon_{b}-\mathscr{L}_{b} \Upsilon_{a}\right) \\
& =f_{a b c}\left(\mathscr{L}_{c} p-\check{\Delta}_{Q} \Upsilon_{c}\right)-D_{1} \check{\Delta}_{Q} \gamma_{a b} \\
& =D_{1}\left(f_{a b c} \Xi_{c}-\check{\Delta}_{Q} \gamma_{a b}\right),
\end{aligned}
$$

we can employ Eq. (3.7) to trivialise the 1-cocycle

$$
\mathscr{L}_{a} \Xi_{b}-\mathscr{L}_{b} \Xi_{a}-f_{a b c} \Xi_{c}+\check{\Delta}_{Q} \gamma_{a b} \in \operatorname{ker} D_{1},
$$

to the effect

$$
\begin{array}{r}
\mathscr{L}_{a}\left(-\left.k_{b}\right|_{\mathcal{O}_{i}^{Q}}+\imath_{b} P_{i}\right)-\mathscr{L}_{b}\left(-\left.k_{a}\right|_{\mathcal{O}_{i}^{Q}}+\imath_{a} P_{i}\right) \\
=f_{a b c}\left(-\left.k_{c}\right|_{\mathcal{O}_{i}^{Q}}+\imath_{c} P_{i}\right)+\imath_{b} \mathscr{L}_{a} P_{i}+\left.\mathscr{L}_{b} k_{a}\right|_{\mathcal{O}_{i}^{Q}}-\imath_{b} \mathrm{~d}\left(\imath_{a} P_{i}\right) \\
=f_{a b c}\left(-\left.k_{c}\right|_{\mathcal{O}_{i}^{Q}}+\imath_{c} P_{i}\right)+\iota_{b}\left(\imath_{a} \mathrm{~d} P_{i}+\left.\mathrm{d} k_{a}\right|_{\mathcal{O}_{i}^{Q}}\right)
\end{array}
$$




$$
=f_{a b c}\left(-\left.k_{c}\right|_{\mathcal{O}_{i}^{Q}}+\imath_{c} P_{i}\right)-\check{\Delta}_{Q} g_{(a b), i} .
$$

This completes the proof of the proposition.

Appendix G. A proof of Proposition 7.4

We have the identity

$$
D_{1} \mathscr{L}_{a} h_{n}=-\check{\Delta}_{T_{n}} \mathscr{L}_{a} p=-\check{\Delta}_{T_{n}}\left(\check{\Delta}_{Q} \Upsilon_{a}+D_{1} \Xi_{a}\right)=-D_{1} \check{\Delta}_{T_{n}} \Xi_{a},
$$

whence

$$
\mathscr{L}_{a} h_{n}+\check{\Delta}_{T_{n}} \Xi_{a} \in \operatorname{ker} D_{1}
$$

Relation (2.29) ensures the triviality of the latter cocycle as it gives

$$
\begin{aligned}
\mathscr{L}_{a} f_{n i} & =-\imath_{a} \check{\Delta}_{T_{n}} P_{i}=-\check{\Delta}_{T_{n}}\left(\imath_{a} P_{i}\right)=-\check{\Delta}_{T_{n}} \Xi_{a}, \\
\mathscr{L}_{a} q_{n i j} & =0
\end{aligned}
$$

and so, indeed,

$$
\mathscr{L}_{a} h_{n}=-\check{\Delta}_{T_{n}} \Xi_{a},
$$

as claimed.

\section{Appendix H. Commuting diagrams For G-Equivariant String BaCKGrounds}

In the appendix, we have gathered the (rather heavily decorated) 2-diagrams whose commutativity expresses the various coherence conditions imposed on elements of a G-equivariant string background.

Thus, we have a diagrammatic representation of

- Eq. 8.3 .

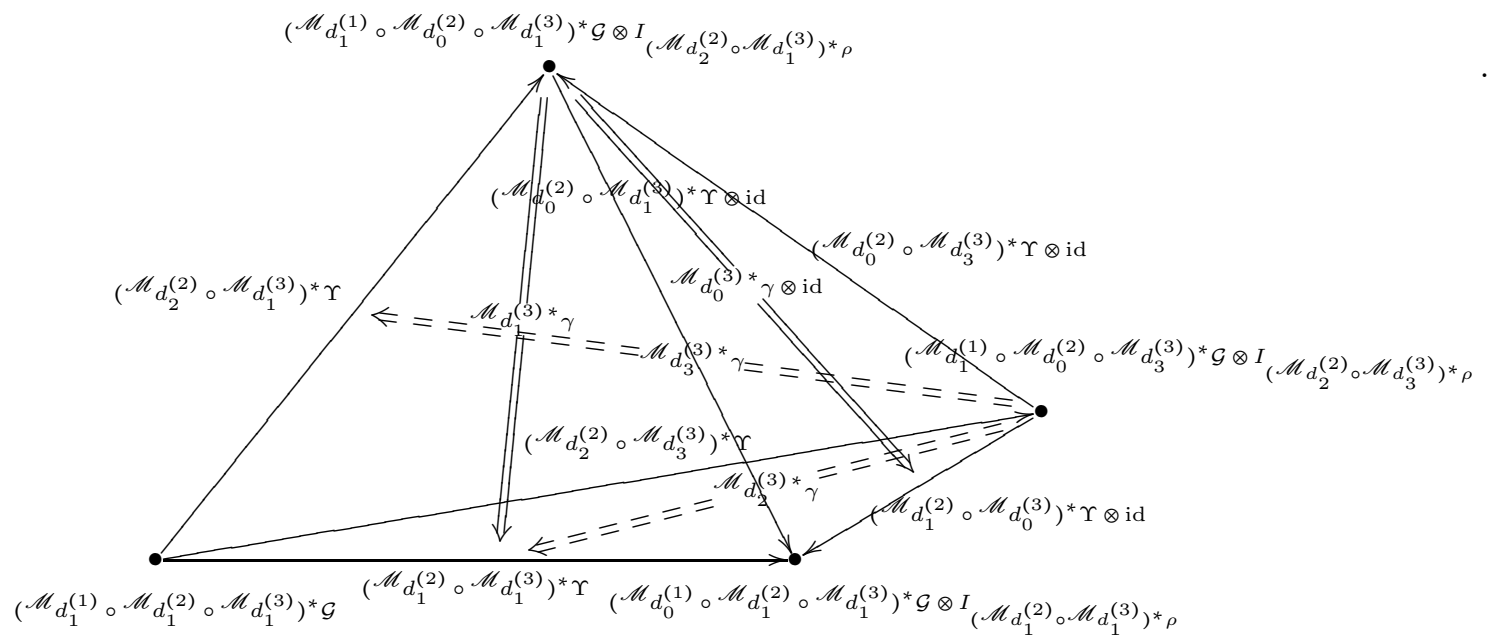


- Eq. 8.4

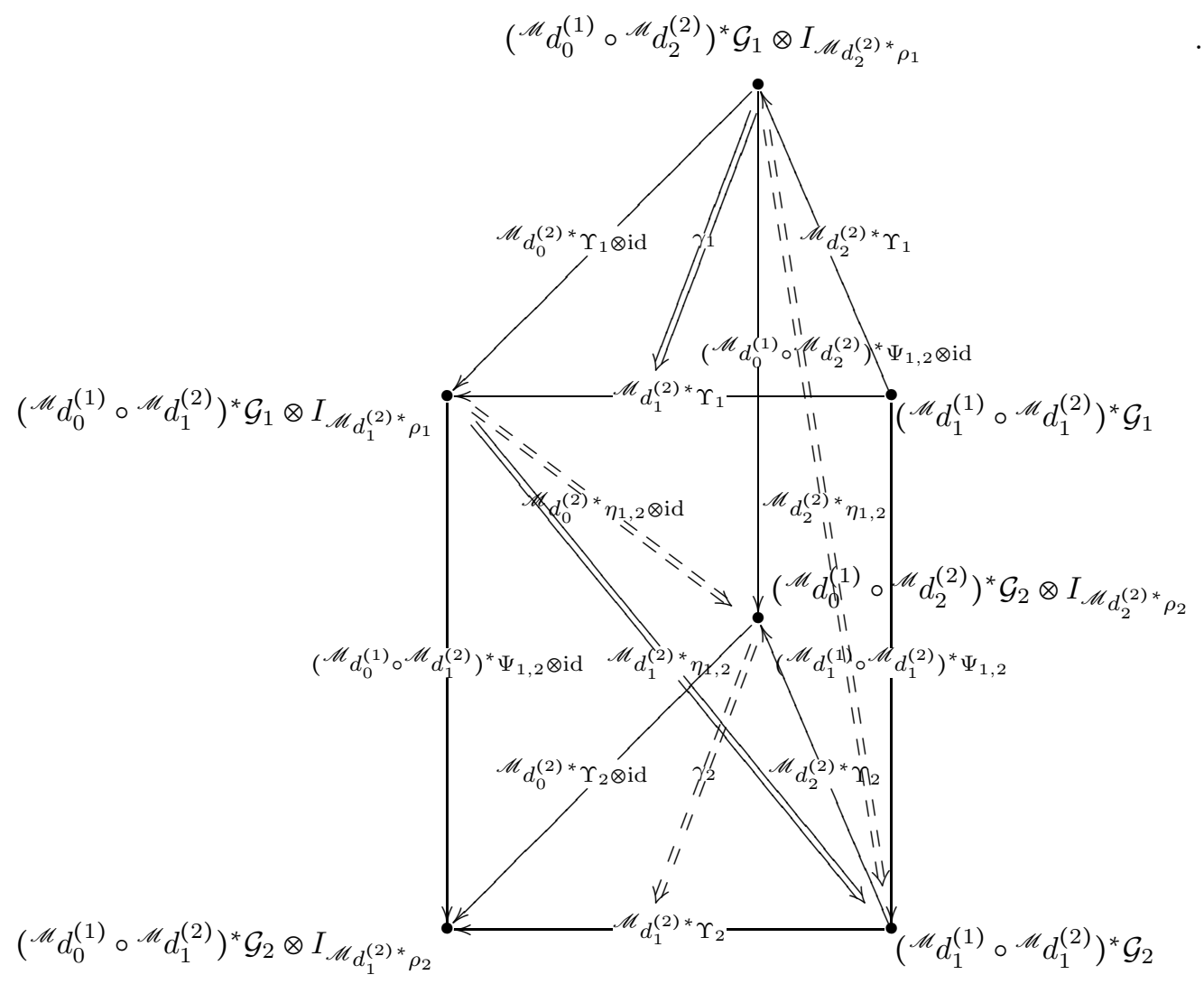

- Eq. 8.5

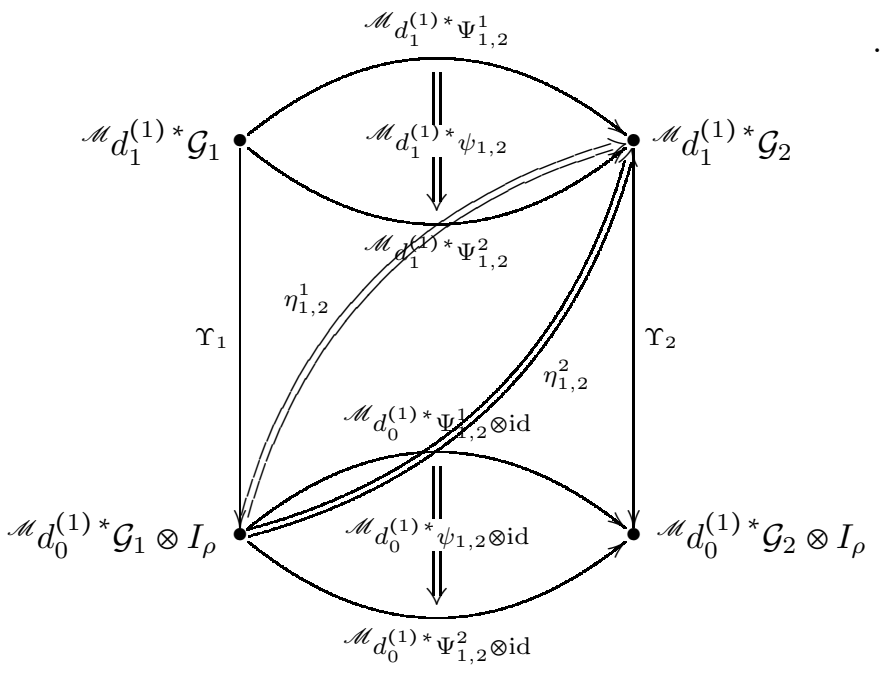


- Eq. 8.8

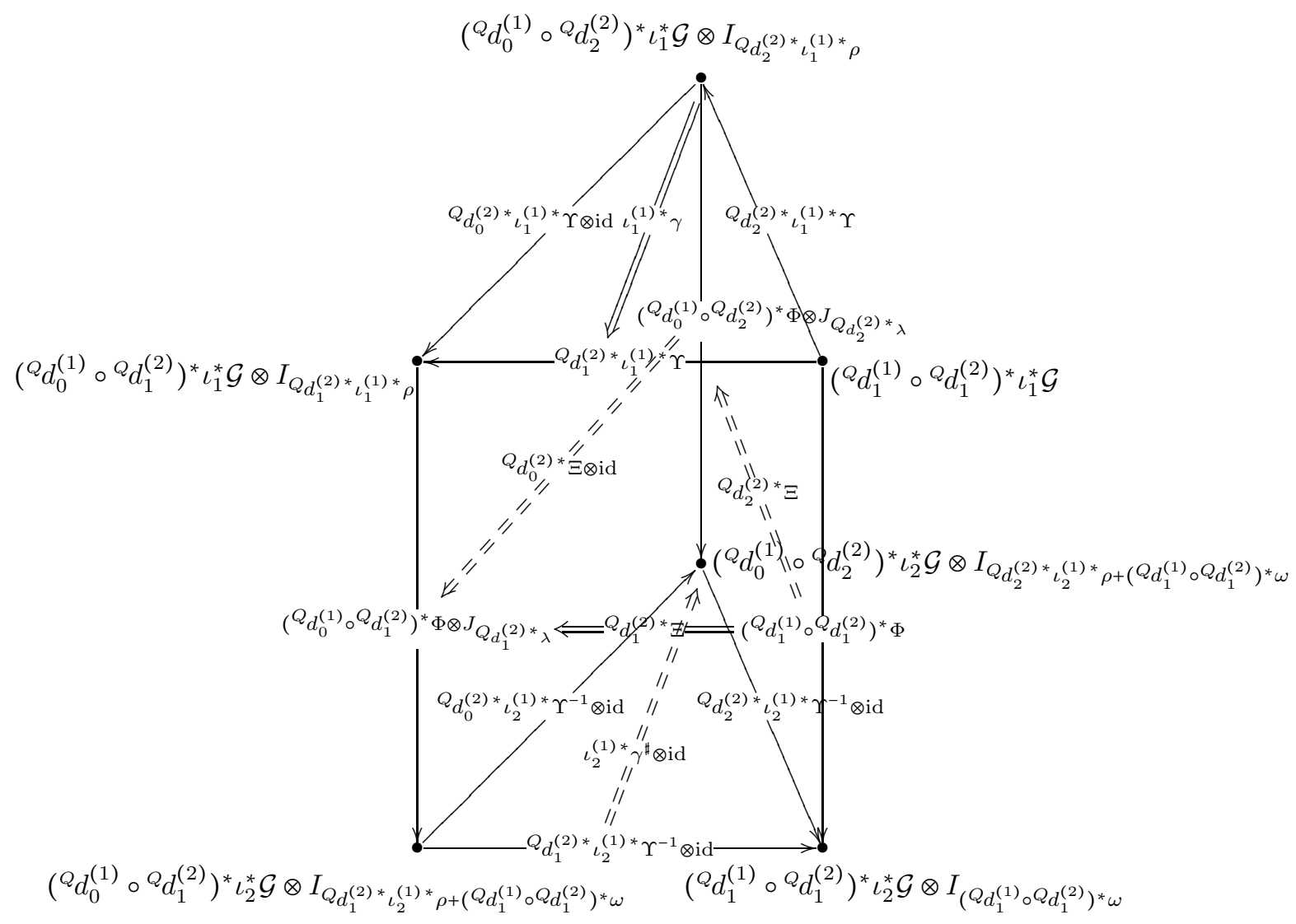

Remark H.1. Note that the first diagram makes sense in virtue of relation (8.6). The only element of the second one that calls for a word of explanation is the left rear face, which represent a slightly more complex structure (we have dropped the obvious gerbe labels of the inner vertices)

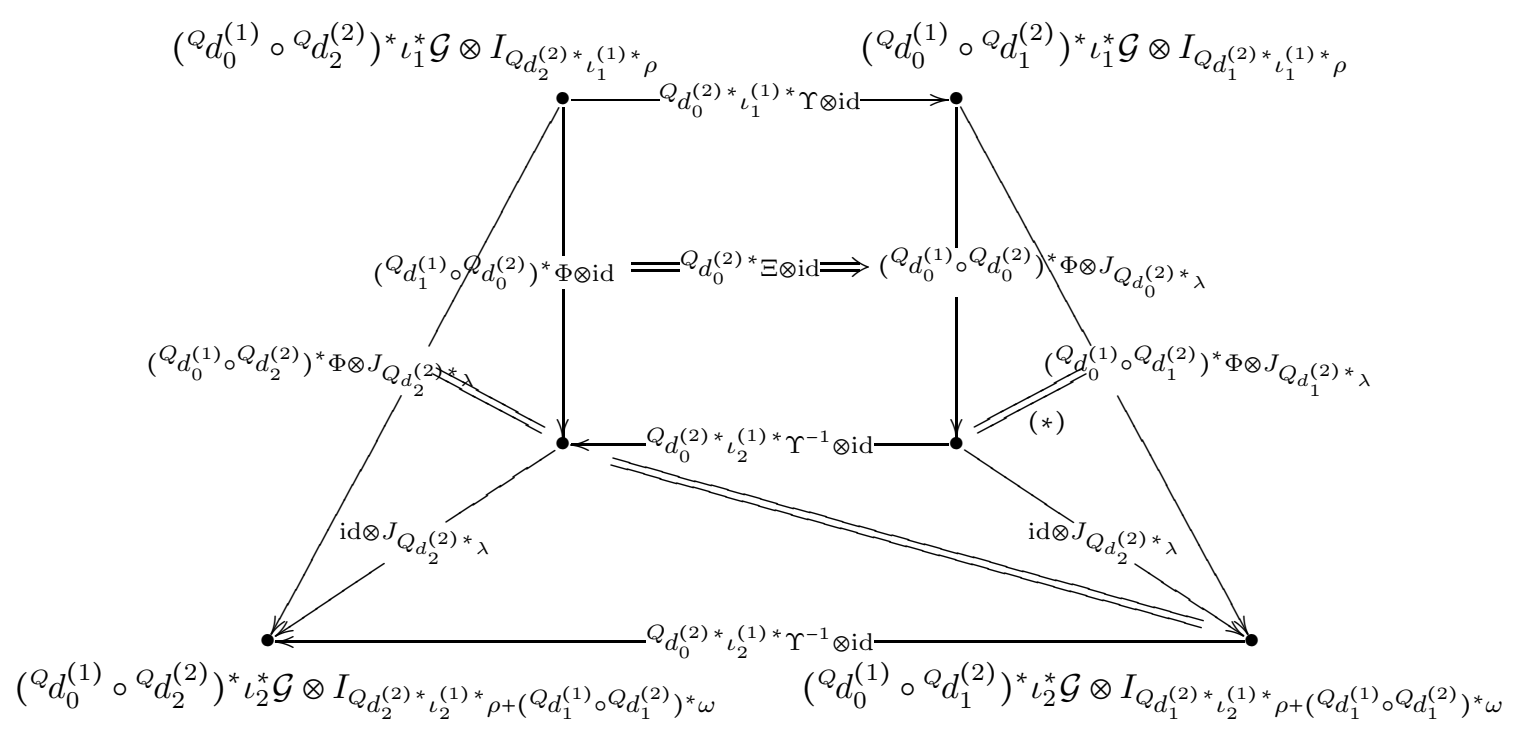

We recover the simplified form of the face upon employing (the pullback of) Eq. (2.41) in conjunction with Eqs. (2.38) and (2.42), the latter giving rise to an identity 2isomorphism for the right triangle in the above subdiagram (indicated by $(*)$ ). 
- Eq. 8.9

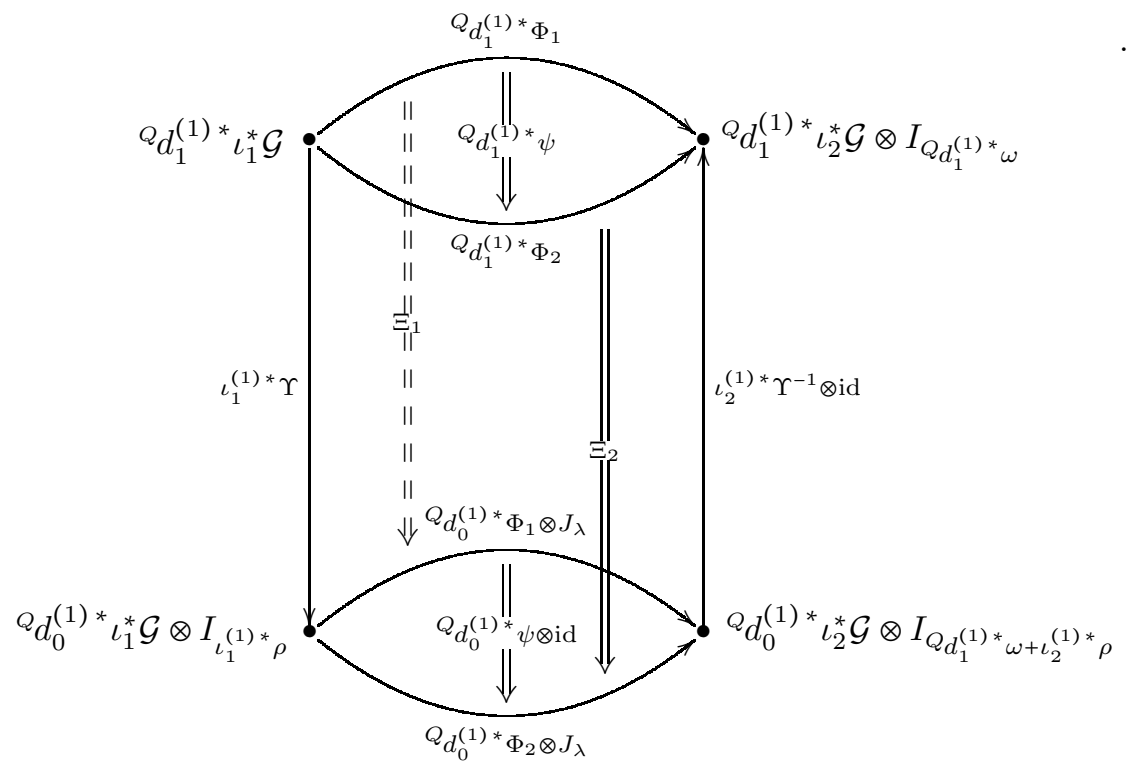

- Eq. 8.11

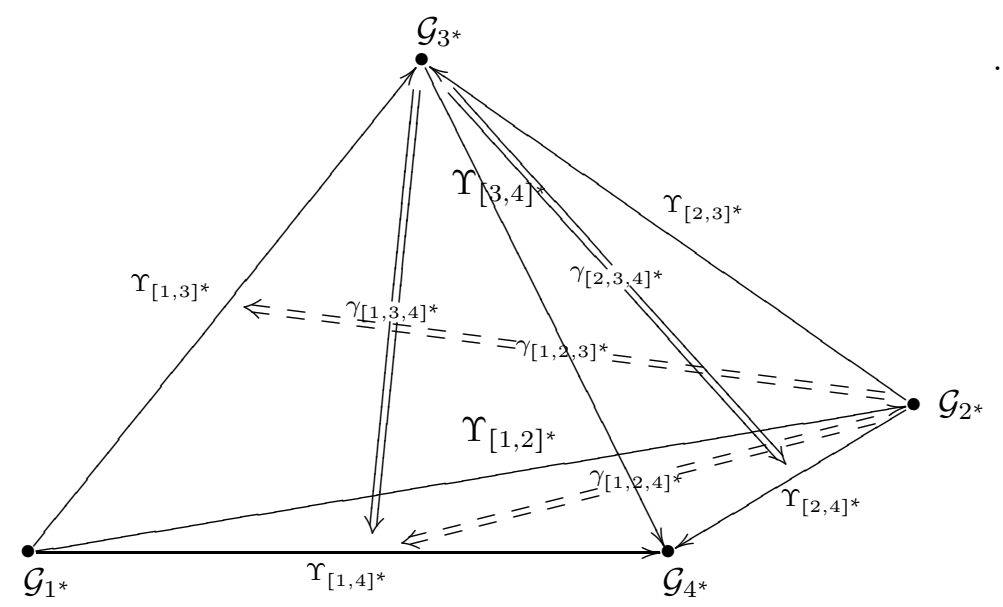


- Eq. (8.12)

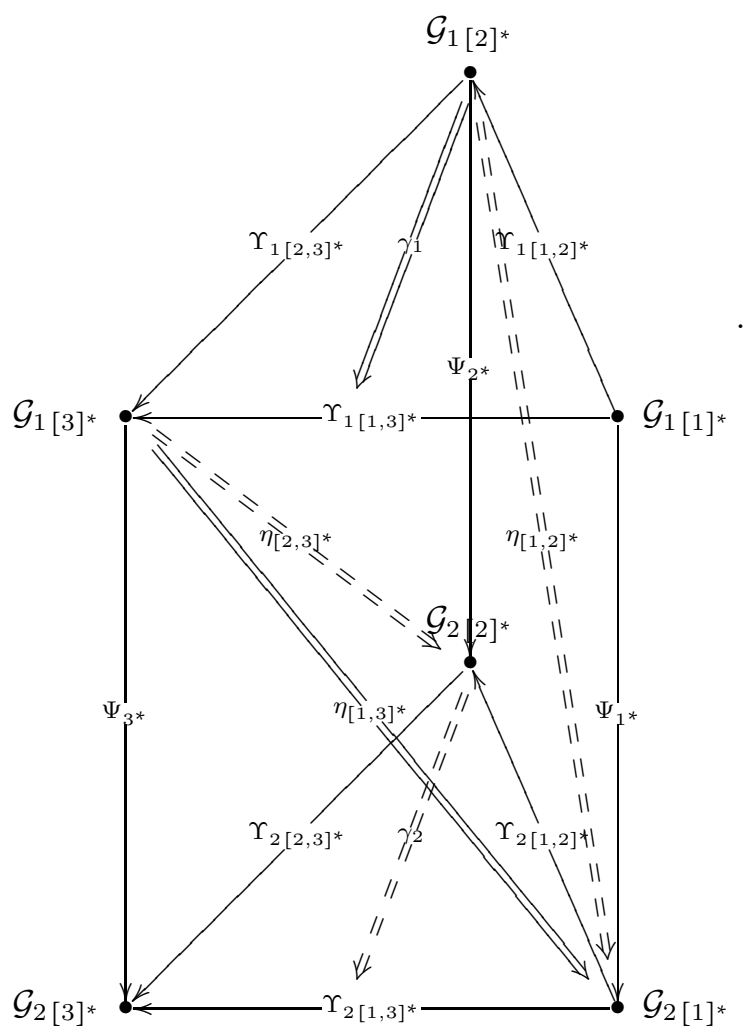

- Eq. (8.13)

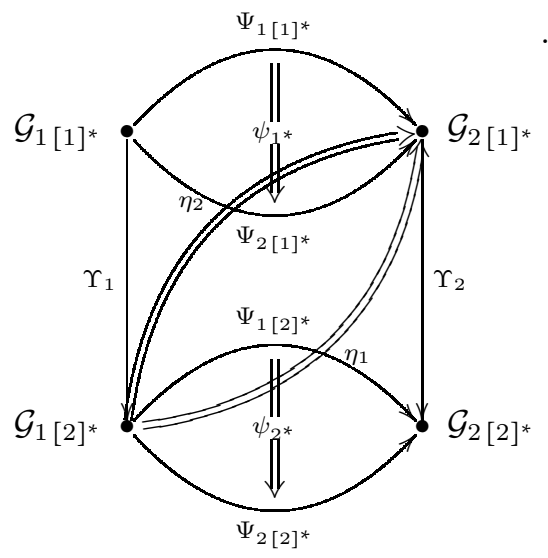

\section{ApPendix I. Natural Simplicial REFinements of OPEN COVERS}

In this appendix, we extend the basic simplicial framework introduced in Section 2.2 with view to applying it in a cohomological classification, carried out in Section 11, of G-equivariant string backgrounds, the latter being regarded of as sheaf-theoretic structures supported by the simplicial G-space G $\mathscr{F}$. In what follows, we use the notation of Section 2.2 .

Let $M: \Delta \rightarrow$ Man be a contravariant functor, so that the family $\left\{M^{m}\right\}_{m=0,1, \ldots}$ of manifolds $M^{m}:=M([m])$, together with the collection of maps $\mu_{k}^{(m+1)}:=M\left(\theta_{k}^{(m+1)}\right): M^{m+1} \rightarrow$ $M^{m}$, forms an incomplete simplicial manifold. Furthermore, let $\left\{\mathcal{O}^{m}\right\}_{m=0,1, \ldots}$ be a sequence of open covers, each $\mathcal{O}^{m}=\left\{\mathcal{O}_{i}^{m}\right\}_{i \in \mathscr{I}^{m}}$ covering the corresponding smooth manifold $M^{m}$ (here, the $\mathscr{I}^{m}$ are index sets). Denote

$$
\Delta^{m}:=\bigcup_{k=0}^{m} \Delta(k, m), \quad \mathcal{I}^{m}:=\bigcup_{k=0}^{m} \mathscr{I}^{k} .
$$


Define an index set

$$
\mathscr{J}^{m}:=\left\{\jmath: \Delta^{m} \rightarrow \mathcal{I}^{m} \quad \mid \quad \theta \in \Delta(k, m) \Rightarrow \jmath(\theta) \in \mathscr{I}^{k}\right\}
$$

and use its elements to label open/8 sets

$$
\mathcal{U}_{\jmath}^{m}:=\bigcap_{k=0}^{m} \bigcap_{\theta \in \Delta(k, m)} M(\theta)^{-1}\left(\mathcal{O}_{\jmath(\theta)}^{k}\right) .
$$

There exists a simple construction, advanced in Ref. [Tu06], of a simplicial sequence of open covers refining a given open cover of a simplicial manifold, which we now review. In so doing, we follow its transcription given in Ref. GW09. We have

Proposition I.1. The collection $\mathcal{U}^{m}:=\left\{\mathcal{U}_{\jmath}^{m}\right\}_{\mathcal{J}} \mathscr{J}^{m}$ of open sets is a refinement of the open cover $\mathcal{O}^{m}$ of $M^{m}$.

Proof. First, we show that $\mathcal{U}^{m}$ is a cover of $M^{m}$. Indeed, for any $x \in M^{m}$ and each $\theta \in$ $\Delta(k, m)$, there exists an index $j_{\theta} \in \mathscr{I}^{k}$ such that $M(\theta)(x) \in \mathcal{O}_{j_{\theta}}^{k}$, and the ensuing assignment $\jmath: \theta \mapsto j_{\theta}, \theta \in \Delta(k, m)$ defines an index $\jmath \in \mathscr{J}^{m}$ such that, clearly, $x \in \mathcal{U}_{\jmath}^{m}$.

Next, we convince ourselves by exploiting the functoriality of $M$, that the new cover is a refinement of the original one: Indeed, $\mathcal{U}_{\jmath}^{m} \subset M\left(\mathrm{id}_{[m]}\right)^{-1}\left(\mathcal{O}_{\jmath\left(\mathrm{id}_{[m]}\right)}^{m}\right) \equiv \operatorname{id}_{M^{m}}^{-1}\left(\mathcal{O}_{\jmath\left(\mathrm{id}_{[m]}\right)}^{m}\right)=$ $\left.\mathcal{O}_{J(\mathrm{id}[m]}^{m}\right)$, and so each $\mathcal{U}_{\jmath}^{m}$ is contained in the corresponding open set $\mathcal{O}_{r(\jmath)}^{m}$ with $r: \mathscr{J}^{m} \rightarrow$ $\mathscr{I}^{m}: \jmath \mapsto \jmath\left(\operatorname{id}_{[m]}\right)$.

In the next step, we verify

Proposition I.2. Let $J: \Delta \rightarrow$ Set be a map between the object and morphism classes of the two categories defined, for arbitrary $\theta \in \Delta(l, m)$ and $\xi \in \Delta(k, l)$, by the respective formula:

$J([m]):=\mathscr{J}^{m}, \quad J(\theta): \mathscr{J}^{m} \rightarrow \mathscr{J}^{l}: \jmath \mapsto J(\theta)(\jmath), \quad(J(\theta)(\jmath))(\xi):=\jmath(\theta \circ \xi) \in \mathscr{I}^{k}$.

Then, $J$ is a contravariant functor which makes the family $\left\{\mathscr{J}^{m}\right\}_{m=0,1, \ldots}$ into an incomplete simplicial set.

Proof. Clearly, $J(\theta) \in \operatorname{Hom}_{\text {Set }}(J([m]), J([l]))$ by the very definition of the morphism component of the map $J$. Besides, for any $\eta \in \Delta(m, p)$ and $\jmath \in \mathscr{J}^{p}$, we find

$(J(\eta \circ \theta)(\jmath))(\xi)=\jmath(\eta \circ \theta \circ \xi)=(J(\eta)(\jmath))(\theta \circ \xi)=(J(\theta)(J(\eta)(\jmath)))(\xi) \equiv((J(\theta) \circ J(\eta))(\jmath))(\xi)$, whence

$$
J(\eta \circ \theta)=J(\theta) \circ J(\eta)
$$

as desired. The remaining part of the statement of the proposition now follows from the one-to-one correspondence between incomplete simplicial sets and contravariant functors $S$ : $\Delta \rightarrow$ Set.

We have a natural

Definition I.3. Let $\left\{M^{m}\right\}_{m=0,1, \ldots}$ be an incomplete simplicial manifold with face maps $\mu_{k}^{(m+1)}: M^{(m+1)} \rightarrow M^{m}$. A sequence $\left\{\mathcal{O}^{m}\right\}_{m=0,1, \ldots}$ of open covers $\mathcal{O}^{m}=\left\{\mathcal{O}_{i}^{m}\right\}_{i \epsilon \mathscr{I} m}$, each $\mathcal{O}^{m}$ covering the corresponding $M^{m}$, is termed simplicial iff the sequence $\left\{\mathscr{I}^{m}\right\}_{m=0,1, \ldots}$ forms an incomplete simplicial set with respect to the collection of face maps $\iota_{k}^{(m+1)}: \mathscr{I}^{m+1} \rightarrow$ $\mathscr{I}^{m}, k=0,1, \ldots, m+1$ and the condition

$$
\mu_{k}^{(m+1)}\left(\mathcal{O}_{i}^{m+1}\right) \subset \mathcal{O}_{\iota_{k}^{(m+1)}(i)}^{m}
$$

is satisfied.

The basic feature of the construction just described is stated in the following

Proposition I.4. The sequence $\left\{\mathcal{U}^{m}\right\}_{m=0,1, \ldots}$ of open covers $\mathcal{U}^{m}=\left\{\mathcal{U}_{\jmath}^{m}\right\}_{\jmath \in \mathscr{J}^{m}}$ of the incomplete simplicial manifold engendered by a contravariant functor $M: \Delta \rightarrow$ Man is simplicial.

\footnotetext{
${ }^{8}$ The sets are open as finite intersections of continuous preimages of open sets.
} 
Proof. The sole thing that has to be checked is identity ([.1). It follows from the more general one:

$$
M(\theta)\left(\mathcal{U}_{\jmath}^{m}\right) \subset \mathcal{U}_{J(\theta)(\jmath)}^{l},
$$

valid for all $\jmath \in \mathscr{J}^{m}$ and an arbitrary map $\theta \in \Delta(l, m)$, upon restricting the latter to the universal coface maps $\theta_{k}^{(m)}$. It therefore remains to prove the above identity, which is tantamount to $M(\theta)\left(\mathcal{U}_{\jmath}^{m}\right)$ being contained in all the open sets $M(\eta)^{-1}\left(\mathcal{O}_{(J(\theta)(\jmath)}^{k}(\eta), k=0,1, \ldots, m, \eta \epsilon\right.$ $\Delta(k, l)$ obtained, with the help of the functor $M$, from some sequence $\left\{\mathcal{O}^{m}\right\}_{m=0,1, \ldots}$ of open covers $\mathcal{U}^{m}=\left\{\mathcal{O}_{i}^{m}\right\}_{i \in \mathscr{I}^{m}}$ of the $M^{m}=M([m])$ in the manner detailed. The desired inclusions are readily inferred from

$$
\begin{aligned}
& M(\eta)\left(M(\theta)\left(\mathcal{U}_{\jmath}^{m}\right)\right)=M(\theta \circ \eta)\left(\mathcal{U}_{\jmath}^{m}\right) \equiv M(\theta \circ \eta)\left(\bigcap_{k=0}^{m} \bigcap_{\psi \in \Delta(k, m)} M(\psi)^{-1}\left(\mathcal{O}_{\jmath(\psi)}^{k}\right)\right) \\
& \subset \bigcap_{k=0}^{m} \bigcap_{\psi \in \Delta(k, m)} M(\theta \circ \eta)\left(M(\psi)^{-1}\left(\mathcal{O}_{\jmath(\psi)}^{k}\right)\right) \subset M(\theta \circ \eta)\left(M(\theta \circ \eta)^{-1}\left(\mathcal{O}_{\jmath(\theta \circ \eta)}^{k}\right)\right) \\
& =\mathcal{O}_{\jmath(\theta \circ \eta)}^{k} \equiv \mathcal{O}_{(J(\theta)(\jmath))(\eta)}^{k} .
\end{aligned}
$$

An obvious question arises at this stage as to the naturality of the above construction of simplicial covers of incomplete simplicial manifolds. The answer to this question is given below.

Proposition I.5. Let $M_{N}: \Delta \rightarrow$ Man, $N=1,2$ be a pair of contravariant functors, so that the respective families $\left\{M_{N}^{m}\right\}_{m=0,1, \ldots}$ of manifolds $M_{N}^{m}:=M_{N}([m])$, together with the corresponding collections of maps $\mu_{N, k}^{(m+1)}:=M_{N}\left(\theta_{k}^{(m+1)}\right): M_{N}^{m+1} \rightarrow M_{N}^{m}$, form incomplete simplicial manifolds. Furthermore, let $\left\{\mathcal{O}_{N}^{m}\right\}_{m=0,1, \ldots, N}, N=1,2$ be sequences of open covers $\mathcal{O}_{N}^{m}=\left\{\mathcal{O}_{N, i}^{m}\right\}_{i \in \mathscr{I}_{N}^{m}}$ of the respective $M_{N}^{m}$, with the corresponding index sets $\mathscr{I}_{N}^{m}$. Finally, let $\left\{\mathcal{U}_{N}^{m}\right\}_{m=0,1, \ldots}, N=1,2$ be the simplicial sequences of refinements $\mathcal{U}_{N}^{m}=\left\{\mathcal{U}_{N, j}^{m}\right\}_{j \in \mathscr{J}_{N}^{m}}$ of the $\mathcal{O}_{N}^{m}$ engendered by the $M_{N}$ and indexed by the corresponding incomplete simplicial sets $\left\{\mathscr{J}_{N}^{m}\right\}_{m=0,1, \ldots,} \mathscr{J}_{N}^{m}:=J_{N}([m])$, the latter being induced by the respective contravariant functors $J_{N}: \Delta \rightarrow$ Set. Every natural transformation $\mu: M_{1} \rightarrow M_{2}$, with $\mu^{(m)}:=\mu([m]) \in$ $\operatorname{Hom}_{\mathrm{Man}}\left(M_{1}^{m}, M_{2}^{m}\right)$, and a sequence $\left\{i^{(m)}\right\}_{m=0,1, \ldots}$ of maps $i^{(m)}: \mathscr{I}_{1}^{n} \rightarrow \mathscr{I}_{2}^{m}$ satisfying the covering relations

$$
\mu^{(m)}\left(\mathcal{O}_{1, i}^{m}\right) \subset \mathcal{O}_{2, i(m)}^{m}(i)
$$

canonically induces a natural transformation $j: J_{1} \rightarrow J_{2}$, with

$$
j^{(m)}:=j([m]) \in \operatorname{Hom}_{\text {Set }}\left(J_{1}^{m}, J_{2}^{m}\right),
$$

satisfying the covering relations

$$
\mu^{(m)}\left(\mathcal{U}_{1, \jmath}^{m}\right) \subset \mathcal{U}_{2, j}^{m}(m)(\jmath)
$$

Proof. Consider a map $j^{(m)}: \mathscr{J}_{1}^{m} \rightarrow \mathscr{J}_{2}^{m}$ assigning to an arbitrary index $\jmath \in \mathscr{J}_{1}^{m}$ a map $\jmath^{(m)} \equiv j^{(m)}(\jmath)$ defined subset-wise over $\Delta^{m}$ by the formulæ

$$
\left.\jmath^{(m)}\right|_{\Delta(k, m)}=i^{(k)} \circ \jmath \text {. }
$$

Clearly, this definition makes sense as

$$
\theta \in \Delta(k, m) \Rightarrow \jmath^{(m)}(\theta)=i^{(k)}(\jmath(\theta)) \in i^{(k)}\left(\mathscr{I}_{1}^{k}\right) \subset \mathscr{I}_{2}^{k} .
$$

Take the universal coface map $\theta_{k}^{(m+1)} \in \Delta(m, m+1)$ and an arbitrary map $\xi \in \Delta(k, m)$. We obtain, for any index $\jmath \in \mathscr{J}_{1}^{m}$,

$$
\begin{aligned}
\left(J_{2}\left(\theta_{k}^{(m+1)}\right)\left(j^{(m+1)}(\jmath)\right)\right)(\xi) & =\left(j^{(m+1)}(\jmath)\right)\left(\theta_{k}^{(m+1)} \circ \xi\right)=i^{(k)}\left(\jmath\left(\theta_{k}^{(m+1)} \circ \xi\right)\right) \\
& =\left(i^{(k)} \circ\left(J_{1}\left(\theta_{k}^{(m+1)}\right)(\jmath)\right)\right)(\xi)=\left(j^{(m)}\left(J_{1}\left(\theta_{k}^{(m+1)}\right)(\jmath)\right)\right)(\xi)
\end{aligned}
$$


which assures the commutativity of the diagram

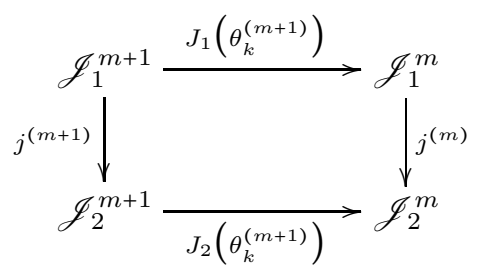

of index maps. Upon expressing an arbitrary map $\theta \in \Delta(l, m)$ in terms of the universal coface maps, we readily infer from the above the naturality of $j$.

At this stage, it remains to check the covering relations ([.4) for the natural transformation constructed. These follow immediately from

$$
M_{2}(\theta)\left(\mu^{(m)}\left(\mathcal{U}_{1, \jmath}^{m}\right)\right)=\mu^{(k)}\left(M_{1}(\theta)\left(\mathcal{U}_{1, \jmath}^{m}\right)\right) \subset \mu^{(k)}\left(\mathcal{O}_{1, \jmath(\theta)}^{k}\right) \subset \mathcal{O}_{2,(i(k) \circ \jmath)(\theta)}^{k}=\mathcal{O}_{2,\left(j^{(m)}(\jmath)\right.}^{k}(\theta),
$$

valid for any $\jmath \in \mathscr{J}_{1}^{m}$ and an arbitrary $\theta \in \Delta(k, m)$ in consequence of the assumed naturality of $\mu$ (ensuring the first equality) and of the very definition of the $\mathcal{U}_{1, j}^{m}$ (explaining the first inclusion), as well as the covering relations ([.2.) (the second inclusion). have

In the context of G-equivariant structures on gerbes and related geometric objects, we

Definition I.6. Whenever the functors $M_{N}, N=1,2$ and the natural transformation $\mu$ between them, appearing in Proposition II.5, are the nerve functors $M_{N}:=\mathrm{G} \mathscr{M}_{N}$ for the action groupoids $\mathrm{G} \ltimes \mathscr{M}_{N}$ of G-spaces $\mathscr{M}_{N}$ and the associated family of G-maps, respectively, we shall call the $\mathcal{U}_{N}^{m}$ aligned simplicial sequences of G-invariant refinements of open covers.

\section{REFERENCES}

[AS05] A.Yu. Alekseev and T. Strobl, "Current algebra and differential geometry", JHEP 0503 (2005), 035.

[CJM02] A.L. Carey, S. Johnson, and M.K. Murray, "Holonomy on D-branes", arXiv preprint: hepth/0204199.

[CJMSW05] A.L. Carey, S. Johnson, M.K. Murray, D. Stevenson, and B.-L. Wang, "Bundle gerbes for ChernSimons and Wess-Zumino-Witten theories", Commun. Math. Phys. 259 (2005), 577-613.

[Cou90] T.J. Courant, "Dirac manifolds", Trans. Amer. Math. Soc. 319 (1990), 631-661.

$[\mathrm{dF}] \quad$ P. de Fromont, "Anomalies globales de jauge dans les modèles WZW et coset de la théorie des champs", 2010 ENS-L internship report, unpublished.

[Dor93] I.Ya. Dorfman, Dirac Structures and Integrability of Nonlinear Evolution Equations, Nonlinear science: Theory and Applications Series, John Wiley \& Sons, 1993.

[FGK88] G. Felder, K. Gawędzki, and A. Kupiainen, "Spectra of Wess-Zumino-Witten models with arbitrary simple groups", Commun. Math. Phys. 117 (1988), 127-158.

[FOM05] J.M. Figueroa-O'Farrill and N. Mohammedi, “Gauging the Wess-Zumino term of a sigma model with boundary", JHEP 0508 (2005), 086.

[FOS94a] J.M. Figueroa-O'Farrill and S. Stanciu, "Equivariant cohomology and gauged bosonic $\sigma$-models", arXiv preprint: hep-th/9407149.

[FOS94b] _ "Gauged Wess-Zumino terms and equivariant cohomology", Phys. Lett. B341 (1994), 153-159.

[FSS96] J.A. Fuchs, B. Schellekens, and C. Schweigert, "The resolution of field identification fixed points in diagonal coset theories", Nucl. Phys. B461 (1996), 371-406.

[FSW08] J.A. Fuchs, C. Schweigert, and K. Waldorf, "Bi-branes: Target space geometry for world sheet topological defects", J. Geom. Phys. 58 (2008), 576-598.

[Gaw88] K. Gawędzki, "Topological Actions in Two-Dimensional Quantum Field Theory", Nonperturbative Quantum Field Theory (G. 't Hooft, A. Jaffe, G. Mack, P. Mitter, and R. Stora, eds.), Plenum Press, 1988, pp. 101-141.

[Gaw05] _ _Abelian and non-Abelian branes in WZW models and gerbes", Commun. Math. Phys. $\mathbf{2 5 8}$ (2005), 23-73.

[GK89a] K. Gawędzki and A. Kupiainen, "Coset construction from functional integrals", Nucl. Phys. B320 (1989), 625-668.

[GK89b] $\quad$, "G/H conformal field theory from gauged WZW model", Phys. Lett. B215 (1989), $119-123$.

[GR02] K. Gawȩdzki and N. Reis, "WZW branes and gerbes”, Rev. Math. Phys. 14 (2002), 1281-1334. 
[GR03] $\quad$, "Basic gerbe over non simply connected compact groups", J. Geom. Phys. 50 (2003), $28-55$.

[GSW08a] K. Gawędzki, R.R. Suszek, and K. Waldorf, "Bundle gerbes for orientifold sigma models", arXiv preprint: 0809.5125 [math-ph], accepted for publication in Adv. Theor. Math. Phys.

[GSW08b] K. Gawędzki, R.R. Suszek, and K. Waldorf, "WZW orientifolds and finite group cohomology", Commun. Math. Phys. 284 (2008), 1-49.

[GSW10] K. Gawędzki, R.R. Suszek, and K. Waldorf, "Global gauge anomalies in two-dimensional bosonic sigma models", Comm. Math. Phys. 302 (2010), 513-580.

[Gua03] M. Gualtieri, "Generalized Complex Geometry", Ph.D. thesis, St John's College, Oxford, UK, 2003.

[GW09] K. Gawȩdzki and K. Waldorf, "Polyakov-Wiegmann formula and multiplicative gerbes", JHEP 09 (2009), 073.

[Hit03] N. Hitchin, "Generalized Calabi-Yau manifolds", Quart. J. Math. Oxford Ser. 54 (2003), 281308.

[Hit06] _ "Brackets, forms and invariant functionals", Asian J. Math. 10 (2006), 541-560.

[Hor96] K. Hori, "Global aspects of gauged Wess-Zumino-Witten models", Commun. Math. Phys. 182 (1996), 1-32.

[HS89] C.M. Hull and B. Spence, "The gauged nonlinear sigma model with Wess-Zumino term", Phys. Lett. B232 (1989), 204-210.

[HS91] C.M. Hull and B.J. Spence, "The geometry of the gauged sigma model with Wess-Zumino term", Nucl. Phys. B353 (1991), 379-426.

[JJMO90] I. Jack, D.R.T. Jones, N. Mohammedi, and H. Osborn, "Gauging the general $\sigma$-model with a Wess-Zumino term", Nucl. Phys. B332 (1990), 359.

[KPSY89] D. Karabali, Q.-H. Park, H.J. Schnitzer, and Z. Yang, "A GKO construction based on a path integral formulation of gauged Wess-Zumino-Witten actions”, Phys. Lett. B216 (1989), 307312.

[LWX98] Z.-J. Liu, A. Weinstein, and P. Xu, "Manin triples for Lie bialgebroids", J. Diff. Geom. 45 (1998), 547-574.

[Mac87] K. MacKenzie, "Lie Groupoids and Lie Algebroids in Differential Geometry", London Mathematical Society Lecture Note Series, vol. 124, Cambridge University Press, 1987.

[MM03] I. Moerdijk and J. Mrčun, Introduction to foliations and Lie groupoids, Cambridge Studies in Advanced Mathematics, vol. 91, Cambridge University Press, 2003.

[Mur96] M.K. Murray, "Bundle gerbes", J. Lond. Math. Soc. 54 (1996), 403-416.

[RS09] I. Runkel and R.R. Suszek, "Gerbe-holonomy for surfaces with defect networks", Adv. Theor. Math. Phys. 13 (2009), 1137-1219.

[RS11a] _ "Affine su(2) fusion rules from gerbe 2-isomorphisms", J. Geom. Phys. 61 (2011), $1527-1552$.

[RS11b] _ "Maximally symmetric defects with junctions in the classical WZW model", in writing.

[Seg68] G.B. Segal, "Classifying spaces and spectral sequences", Inst. Hautes Études Sci. Publ. Math. 34 (1968), 105-112.

[SSW07] U. Schreiber, C. Schweigert, and K. Waldorf, "Unoriented WZW models and holonomy of bundle gerbes", Commun. Math. Phys. 274 (2007), 31-64.

[Ste00] D. Stevenson, "The Geometry of Bundle Gerbes", Ph.D. thesis, The University of Adelaide, Australia, 2000.

[Sus11] R.R. Suszek, "Defects, dualities and the geometry of strings via gerbes. I. Dualities and state fusion through defects", Hamburger Beiträge zur Mathematik Nr.360 (2011) [arXiv preprint: 1101.1126 [hep-th]].

[Sus12] _ _ "Defects, dualities and the geometry of strings via gerbes, II. Generalised geometries with a twist", in writing.

[ŠW01] P. Ševera and A. Weinstein, "Poisson geometry with a 3-form background", Prog. Theor. Phys. Suppl. 144 (2001), 145-154.

[SY90a] A.N. Schellekens and S. Yankielowicz, "Field identification fixed points in the coset construction", Nucl. Phys. B334 (1990), 67-102.

[SY90b] _ _Simple currents, modular invariants and fixed points", Int. J. Mod. Phys. A5 (1990), 2903-2952.

[Tu06] J.-L. Tu, "Groupoid cohomology and extensions", Trans. Amer. Math. Soc. 358 (2006), 47214747 .

[Wal07a] K. Waldorf, "Algebraic Structures for Bundle Gerbes and the Wess-Zumino Term in Conformal Field Theory", Ph.D. thesis, Universität Hamburg, 2007.

[Wal07b] _ "More morphisms between bundle gerbes", Theory Appl. Categories 18 (2007), 240-273.

[Wal10] _ "Multiplicative bundle gerbes with connection", Differential Geom. Appl. 28 (2010), 313-340.

[Wei94] C.A. Weibel, An Introduction to Homological Algebra, Cambridge Studies in Advanced Mathematics, no. 38, Cambridge University Press, 1994.

[Wit84] E. Witten, "Non-abelian bosonization in two dimensions", Commun. Math. Phys. 92 (1984), 455-472.

[Wit92] _ "On holomorphic factorization of WZW and coset models", Commun. Math. Phys. 144 (1992), 189-212. 
[Wu93] S.-Y. Wu, "Cohomological obstructions to the equivariant extension of closed invariant forms", J. Geom. Phys. 10 (1993), 381-392.

K.G.: Laboratoire de Physique, C.N.R.S., EnS-Lyon, Université de Lyon, 46 Allée d'Italie, 69364 LyOn, France

E-mail address: kgawedzk@ens-lyon.fr

R.R.S.: Katedra Metod Matematycznych Fizyki, Wydzią Fizyki Uniwersytetu Warszawskiego, Ul. Hoża 74, PL-00-682 Warszawa, Poland

E-mail address: suszek@fuw.edu.pl

K.W.: Fakultät für Mathematik, Universität Regensburg, Universitätsstrasse 31, 93053 ReGensburg, Germany

E-mail address: konrad.waldorf@mathematik. uni-regensburg.de 